A Comparison of Nuclear Effects in High Energy Leptonic and Hadronic Scattering

by

Claude David Rees

A dissertation submitted in partial fulfllment of the requirements for the degree or

\title{
Doctor of Philosophy
}

University of Washington

1984 


\section{University of Washington}

\section{Abstract}

A COMPARISON OF NUCLEAR EFFECTS IN HIGH ENERGY LEPTONIC AND HADRONIC SCATTEKING

\section{By Claude David Rees}

Chairperson of the Supervisory Committee: Professor H.J.Lubatti Department of Physics

A neutrino-Neon (antineutrino-Neon) scattering experiment using the Fermilab 15 foot bubble chamber for analysis is compared with a pi plus- (pi minus)-Neon scattering experiment at 30 and $64 \mathrm{GeV} / \mathrm{c}$ in the CERN BEBC bubble chamber. The comprison analy zes nuclear effects, excluding the EMC effect.

The Fermilab neutrino experiment (E546) used a Ne- $\mathrm{H}_{2}$ (47\%atomic $\mathrm{Ne}$ ) mixture at 29.6 degrees Kelvin and a density of $0.56 \mathrm{gm} / \mathrm{cc}$. Charged current events had the muon identifled by the External Muon Identifler. The average neutrino energy was $90 \mathrm{GeV}$, and the ratio of neutrino to antineutrino induced events was approximately 6:1. All events were fully measured.

The CERN pion experiment (WA51) used a $\mathrm{Ne}-\mathrm{H}_{2}(60 \%$ atomic $\mathrm{Ne}$ ) mixture at 28.15 degrees Kelvin and a density of $0.71 \mathrm{gm} / \mathrm{cc}$. The $36 \mathrm{~K}$ pictures obtained were fully scanned, snd positive, negative, identifled proton, and "straight" track multiplicities recorded for all accepted events. A sample of approximately 1200 events at $30 \mathrm{GeV} / \mathrm{c}$ had all charged tracks measured.

Average multiplicity and dispersion are linearly related. Identifled proton multiplicities agree well with the prediction of the Andersson model. Multiplicity correlations with identifled protons sre studied. KNO scaling distributions, fits to multiplicities as functions of $W^{2}$ and $Q^{2}$, and the behavior of various moments with energy are presented. Correlations between variables are studied, ratios of nuclear to nonnuclear production, and inclusive distributions are compared for $\mathrm{pi}-$ and neutrino- $\mathrm{Ne}$ for different $Q^{2}$. An excess of particles in the backwards hemisphere is seen in both the ratio of nuclear to nonnuclear multiplicities and in twoparticle rapidity correlations, presumably from nuclear reinteractions. In neutrino-Neon, searches for coherent interactions and a nucleon resonance are reported.

Using an isospin subtraction technique, protons too fast to be identifled by ionization are statistically identified, their rapidity distribution derived and compared with the LUND model prediction. There are approximately 0.8 such protons per event, with roughly half from the primary vertex and half from nuclear effects. Results are consistent with the flavor dependence of neutrino scattering, with diquark breakup. 
TABLE OF CONTENTS

Chapter 1. Introduction . . . . . . . . . . . . . 1

1.1. Basic Background. . . . . . . . . . . . . . . . . 1

1.2. Some frequently used symbols. . . . . . . . . . . . 6

Chapter 2. Theoretical background . . . . . . . . . . . . . . 15

2.1. Elementary particles. . . . . . . . . . . . . . . . 15

2.2. The ElectroWeak Interaction. . . . . . . . . . . . 21

2.3. The strong interaction. . . . . . . . . . . . . . . . 34

2.4. High Energy Neutrino Interactions. . . . . . . . . . 43

2.5. Nuclear Effects. . . . . . . . . . . . . . . . . . . 49

Chapter 3. Experiment E546 . . . . . . . . . . . . . . . . . .73

3.1. The experiment run, apparatus, and event reconstruction. 73

3.2. Neutrino energy correction. . . . . . . . . . . . . . 78

Chapter 4. Experiment WA51 . . . . . . . . . . . . . . .85

4.1. The experiment run and event scanning. . . . . . . . 85

4.2. Corrections to the scan data. . . . . . . . . . . . . 88

4.3. Messured events. . . . . . . . . . . . . . . . . . . .94

Ghopter 6, Amalyais Techniques . . . . . . . . . . . . . . 88

6.1. Comparison of $\left(\bar{\nu}^{\prime}\right.$ and $\pi^{ \pm}$incident. . . . . . . . . . 98

5.2. The NUCTST algorithm. . . . . . . . . . . . . . . 100
6.3. $W^{2}$ weighting. . . . . . . . . . . 105

Chapter 6. Multiplicity Results . . . . . . . . . . . . . . . . 108

8.1. Chapter Overview. . . . . . . . . . . . . . . . 108

6.2. Shower track multiplicity distributions. . . . . . . . 110

6.3. Proton multiplicity distributions. . . . . . . . . . 118

6.4. Multiplicity correlations between identifled protons and shower tracks. . . . . . . . . . 134

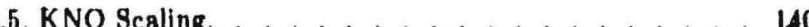

B.6. Features of multiplicity distributions as functions of leptonic and hadronic event variables in $\left(\bar{\nu}^{\prime}\right) \mathrm{Ne} . \ldots . .148$

Chapter 7. Kinematic Distribution Results . . . . . . . . . . 182

7.1. Chapter overview. . . . . . . . . . . . . 102

7.2. General Kinematic Distributions and Correlations between Kinematic Variables. . . . . . . . . . . . . . . . 100

7.3. Comparison of incluaive distrlbutions in $(\tilde{\nu}) \mathrm{Ne}$ and $\pi^{ \pm} \mathrm{Ne} . \quad 179$

7.4. Nuclear particle production a function of rapidity. . . 199

7.5. Study of fast proton $\left(p f_{\text {aot }}\right)$ production. . . . . . . 208 Chapter 8. Other Results . . . . . . . . . . . . . . . . 225

8.1. Chapter Overview. . . . . . . . . . . . . . . . . 225

8.2. Two particle rapidity correlations. . . . . . . . . . 227

8.3. Coherent Scattering. . . . . . . . . . . . . . . 241

8.4. Search for $\Delta_{\text {fatt }}^{+}$in $\nu$ Ne. . . . . . . . . . . . . . . 268

Bibliography. . . . . . . . . . . . . . . . . 208 
Appendix A. Calculation of $(\nu)$ in the impact parameter representation. . . . . . . . . . . . . . . . . 271

Appendix B. Notation Conrentions.

. 274
LIST OF TABLES

Number

Page

3.2.1 Comparison of Bonn and $p_{T}^{\text {bal }}$ energ correction techniques using the LUND monte-carlo. . . . . . . . . . . . . . . .81

4.2.1 Analy zed erents in WA51, showing corrections. . . . . . . 84

6.2.1 Average multiplicities and dispersions. . . . . . . . . . 114

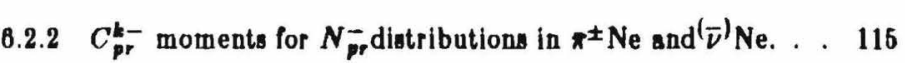

6.2.3 $R_{A}$ for $\pi^{ \pm} \mathrm{Ne}$ and $\left(\bar{\nu}^{\prime} \mathrm{Ne}\right.$ at 30 and $84 \mathrm{GeV} / \mathrm{c}$. . . . . 117

0.3.1 Average multiplicity and dispersion for identlfled protons $N_{p}$ in ${ }^{ \pm}$Ne. . . . . . . . . . . . . . . . 121

0.3.2 Average multiplicity and percentage of minimum-ionizing positive tracks for fast protons for $\pi^{ \pm} \mathrm{Ne}$ and $\left(\bar{\nu}^{\prime} \mathrm{Ne} \ldots \ldots . . .128\right.$

0.6.1 Results of fits to the form $\left\langle N^{\text {ch }}\right\rangle=a+b \cdot \ln \left(W^{2}\right)$ for various scattering systems. . . . . . . . . . . . . . . . . . 152

6.8.2 Results of fits to the form $\left\langle N^{-}\right\rangle=a+b \cdot \ln \left(W^{2}\right)$ for $\left(\bar{\nu}^{\prime}\right)$ Ne and $\left(\bar{v}_{p}{ }_{\mathrm{N}} \quad \ldots \ldots \ldots \ldots \ldots\right.$

0.6.3 Results of fits to the form $\left(N^{+}\right)=a+b \cdot \ln \left(W^{2}\right)$ for $\left(\bar{\nu}^{-}\right) \mathrm{Ne}$ and '

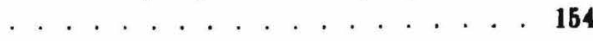

6.0.4 Resuits of fits to the form $\left.\left\langle N^{\text {ehower }}\right\rangle=a+Q^{2} \operatorname{for}^{(} \bar{\nu}\right) \mathrm{Ne} .157$

8.2.1 Fits of rapidity gap distributions to $n(r)=a e^{b r} \ldots \ldots$. . 939 


\section{LIST OF FIGURES}

\section{Number}

2.1.1 Elementary particles. . . . . . . . . . . . . . . . 20

2.2.1 An exsmple of single $\boldsymbol{W}$-exchange. . . . . . . . . . . . 33

2.3.1 $e^{+} e^{-} \rightarrow$ hadrons via a virtual photon. . . . . . . . . 3B

2.3.2 Qusrk confinement from s perfect racuum color dielectric. . 38

2.3.3 The $J^{P}=0^{-}$and $J^{P}=1^{-}$meson $(q \bar{q})$ octets and corresponding singlets. . . . . . . . . . . 41

2.3.4 The $J^{P}=\frac{1}{6}^{+}$baryon (qqq) octet and singlet, and the $J^{P}=\frac{8}{2}^{+}$ baryon decuplet. . . . . . . . . . . . . . . 42

2.4.1 High energy neutrino charged current (CC) diagram. . . . 45

2.6.1 Schematic nuclear reinteraction diagrams. . . . . . . . 48

2.5.2 Nuclear reinteractions for the Cascade and Diffractive Excitation Models. . . . . . . . . . . . . . . .5B

2.5.3 Collective Tube Model picture of nuclear interaction. . . . 01

2.5.4 Quark model picture of nuclear interaction. . . . . . . 65

3.1.1 E546 neutrino beam energy spectrum. . . . . . . . . . .75

3.1.2 EMI layout and bubble chamber. . . . . . . . . . . . 77

6.2.1 Computing the probability the sign of the charge is wrong from a Gaussian error distribution in curnture. . . . . 103

0.2.1 The dlsperslon $D_{p r}^{-}$versus average multiplicity $\left\langle N_{p r}^{-}\right\rangle$. . 112

6.2.2 The evolution of $R_{A}$ with energy for $\pi^{ \pm}$Ne sad $\left({ }^{\prime}\right)$ Ne. . . 119
6.3.1 Normalized multiplicity distribution of identifled protons $\left(N_{p}\right)$.

$$
\text { . . . } 123
$$

6.3.2 An example of proton production in the Andersson model. 125

6.3.3 Arerage number of fast protons $\left\langle N_{p}^{\text {fact }}\right\rangle$ as a function of $N_{p}$ for $\boldsymbol{x}^{ \pm} \mathrm{Ne}$ and $(\bar{\nu}) \mathrm{Ne} . \ldots . . . . . . .130$

6.4.1 Average multiplicity of produced negative particles $\left\langle N_{p r}^{-}\right\rangle_{\text {as a }}$ function of the identifled protons $N_{\mathrm{p}} \ldots \ldots \ldots . .132$

6.4.2 Dispersion $D_{p}^{-}$as a function of $N_{p}$. . . . . . . . . 133

B.4.3 The dispersion in $N_{\mathrm{pr}}\left(D_{\mathrm{pr}}^{-}\right)$for all neutrino data. . . . . 134

6.4.4 $R_{A}(\pi)$ and $\tilde{R}_{A}^{(2)}(\nu)$ versus $N_{p}$ for $\pi^{ \pm} \mathrm{Ne}$ and $\left(\bar{\nu}^{\prime} \mathrm{Ne} . \ldots . \quad 136\right.$

0.4.5 Average number of "effective" collisions $\langle\nu\rangle$ versus $N_{p}$. . . 138

6.4.6 Normalized moments $C_{p r}^{2-}$ and $C_{p r}^{8-}$ for $N_{p r}^{-}$for $\pi^{ \pm}$Ne as a function of $N_{p} \ldots \ldots \ldots \ldots$

6.6.1 The KNO scaling distribution for shower particles comparing different hadronic CM energies. . . . . . . . . . . . 14

6.5.2 The KNO scaling distribution for shower particles for nuclear, non-nuclear, and total algorithms. . . . . . . . . . 145

6.5.3 Comparison of the KNO scaling curves for shower particles between

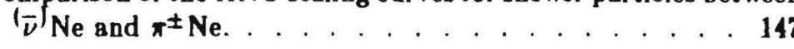

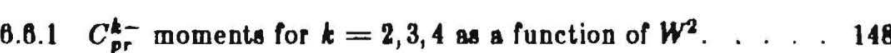

6.0.2 $W^{2}$ dependence of $D_{p r}^{-}, \gamma_{p r}^{-}, f_{p r}^{2--}$, and $f_{p r}^{8---}$ in $(-)$ Ne. . 150

$7.2 .1 \frac{1}{\sigma^{i n o l}} \frac{d}{d\left(W^{i}\right)}$ for nonnuclear $($ NUCTST $=-2)$, nuclear $($ NUCTST $=$ $+2)$, and all events $(N U C T S T=0) . \ldots . . . .185$

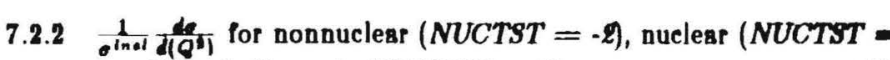
$+2)$, and all events $(N U C T S T=0) . \ldots 160$ 


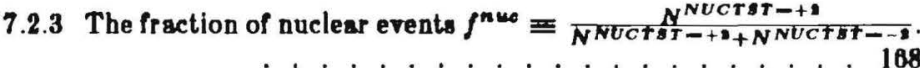

7.2.4 Proportionsl dot plots showing kinematic correlations. . . 170

7.2.5 Correlation plots showing average and dispersion. . . . . 173

7.3.1 Definition of quantities for the "parallel configuration" . . 181

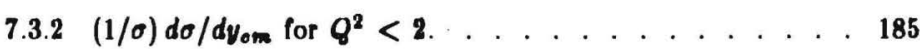

7.3.3 $(1 / \sigma) d \sigma / d y_{c m}$ for $2<Q^{2}<10 \ldots . . . . . . .186$

$7.3 .4(1 / \sigma)$ do/dyom for $10<Q^{2}<30$. . . . . . . . . 187

$7.3 .5(1 / \sigma) d \sigma / d y_{c m}$ for $Q^{2}>30 \ldots \ldots . \ldots . \ldots 188$

$7.3 .0(1 / \sigma) \mathrm{d \sigma} / \mathrm{d} \boldsymbol{s}_{\mathrm{om}}$ for $Q^{2}<2 . \ldots . \ldots 180$

7.3.7 $(1 / \sigma) d \sigma / d s_{0 m}$ for $2<Q^{2}<10 \ldots \ldots 1$

$7.3 .8(1 / \sigma) \mathrm{d} \sigma / \mathrm{d} \mathrm{s}_{\mathrm{cm}}$ for $10<Q^{2}<30$. . . . . . . . . . 192

$7.3 .9(1 / \sigma) \mathrm{d \sigma} / \mathrm{ds} \mathrm{s}_{\mathrm{cm}}$ for $\boldsymbol{g}^{2}>30 \ldots \ldots . \ldots 193$

7.3.10 $(1 / \sigma) d \sigma / d x$ f for $Q^{2}<2 \ldots \ldots$. . . . . . . . . 194

7.3.11 $(1 / \sigma) d \sigma / d x p$ for $2<g^{2}<10 \ldots \ldots . \ldots . . \ldots 195$

$7.3 .12(1 / \sigma) d \sigma / d \varepsilon_{f}$ for $10<Q^{2}<30 \ldots . . . . . .196$

7.3.13 $(1 / \sigma) d \sigma / d x$ for $Q^{2}>30 \ldots \ldots 197$

7.4.1 Morrison results for $R_{A}^{-}$as a function of V/eb and $\nu$ in $D N e .199$

7.4.2 E540 results for $R_{n u c}(v)$ as a function of $y_{\text {lab }}$ and $\nu$. . . 202

7.4.3 E546 results for $R_{\text {nuc }}(y)$ as a function of $y_{\text {lab }}$ and $Q^{2}$. . 204

7.4.4 E546 results for $R_{\text {nuc }}(y)$ as a function of $y_{l a b}$ and $W^{2}$. . . 208

7.5.1 The fast proton ylab distribution.
7.5.2 The identifled proton $y_{\text {lab }}$ distribution for $\pi^{ \pm} \mathrm{Ne}$ and $(\bar{\nu}) \mathrm{Ne} . \quad 214$

7.6.3 The total proton y/ab distribution. . . . . . . . . 215

7.5.4 The LUND model prediction compared with $(\bar{\nu})$ Ne. . . . . 218

7.5.5 Comparison of $p_{\text {faot }}$ Vlab for nuclear $($ NUCTST $=+2)$ and nonnuclear $(N U C T S T=-2)$ events for $\Delta \Phi(1) \ldots \ldots .219$

7.6.6 The ylab P paot distribution in $\left(\bar{\nu}^{\prime}\right)$ Ne as a function of $W^{2}$. 220

7.5.7 The maximum proton lab rapidity for given $W^{2} \ldots$. . . 222

8.2.1 Distributions for the diagonal of the two-particle correlation matrix, $R\left(y_{1}, y_{2}=y_{1}\right) \ldots \ldots \ldots 231$

8.2.2 Two particle correlations $R\left(y_{1}, v_{8}\right) \ldots \ldots \ldots . \ldots . . \ldots 232$

8.2.3 The rapidity gap distribution. . . . . . . . . . . . . 237

8.3.1 Definition of quantities used in the coherent event snalysis. 241

8.3.2 Distributions in $|t|$ and $\left|t-t_{\min }\right|$ in $\nu$ Ne. . . . . . . 240

8.3.3 Distributions in $|t|$ for $D N$ Ne. . . . . . . . . . . . . 248

8.3.4 Distributions in $\left|t-t_{\min }\right|$ for single nucleon $W^{ \pm}$diffraction. 251

8.4.1 The LUND prediction for the invariant thas distribution of $\Delta_{\text {faet }}^{++}$ with cuts . . . . . . . . . . . 257

8.4.2 The $\mathbf{E} 540 \nu \mathrm{Ne}$ invariant mass distribution of $\Delta_{\text {faot }}^{+}$in nonnuclear events, with cuts. . . . . . . . . . 259 


\section{ACKNOWLEDGMENTS}

I thank the physiciats and staff of the Visual Techniques Laboratory for invaluable support, discussions, and guidance in completing this work. In particular I would like to thank Dr. H.J. Lubatti, who acted as advisor for this dissertation, for his patience, interest, and guidance.

\section{DEDICATION}

This dissertation is dedicated to my parents, whose constant concern, interest, moral support, understanding, and high principles have acted as a guiding light throughout my life. 


\section{CHAPTER 1}

\section{INTRODUCTION}

\section{1.1. Bagic Background.}

This dissertation deals with the comparison of nuclear effects in high energy leptonic and hadronic scattering. When an incident particle interacts in a nucleus, the secondary system produced in the interaction can then interact with constituents in the remainder of the nucleus. These reinteraction effects are our subject of inquiry, as well as effects involving the nucleus as a whole.

(The nucleus may have another influence different from such reinteraction or group interaction effects. Nuclei are usually considered to be a superposition state of nucleons $[1]$, described by some wave function $\boldsymbol{\Psi}\left(\overrightarrow{\mathbf{P}}_{1}, \mathbf{F}_{2}, \ldots, \mathbf{F}_{i}, \boldsymbol{\theta}_{1}, \theta_{2}, \ldots, \theta_{i}, \ldots\right)$, where the $\overrightarrow{\mathbf{F}}_{i}$ are the coordinates of the constituent nucleons and the $\theta_{i}$ represent other quantum numbers of these nucleons, such as spin, isospin, etc. In addition nuclear models often include an effective field of exchanged virtual pions as a model of the nuclear binding force ${ }^{|2|}$; but the nucleons are considered to be the same as free nucleons. Because nucleons are composite objects, this is not necessarily the case. Squeezing nucleons together in a nucleus might very well alter the properties of their constituents.

(There is recent evidence of just such an effect ${ }^{[3]}[4]$. Experimental results show that the structure function $F_{2}(x)$, which can in turn be related to the momentum distributions of constituent quarks, is different for nucleons in nuclei than for free nucleons, and in a manner opposite to any difference coming from Fermi momentum alone ${ }^{|5|}$. It has been suggested that this effect can be explained by including scattering from the virtual $\pi$ field of the nucleus ${ }^{|6|}$; however, careful calculations with realistic models for this scenario evidently rule this out ${ }^{|7|}$. Currently there is no good explanation of this "EMC" effect (for European Muon Collaboration ${ }^{|8|}$, the discoverers of the effect). It seems entirely reasonable, however, that some such effect could arise from superposition phenomena of quark wave functions. In short, free nucleons and nucleons in nuclei would not be the same.

(Such an EMC effect is outside the scope of this dissertation, and will not be dealt with here.)

Given that nucleons are constituent objects themselves, and that the mechanism of hadronization of constituents is not very well understood even in particle-nucleon scattering, one might well ask, "Why go to the added complication of using a nuclear target? Won't it just confuse the situation more, and make the interaction hopelessly complex?" 
Perhaps the first response to such \& question should be that any well-defined problem is a valid subject of scientific inquiry; the fact that such a problem is not well understood perhaps cries for more inquiry, not less. Beyond such a purely philosophical reply, there are entirely practical reasons to investigate high energy nuclear interaction phenomena. The very complexity of the problem may be a boon in disguise; perhaps some overall statistical model becomes valid in such a many-interaction picture, which can in turn be both simpler and very revealing of the properties of the interaction and matter in general (for example, the statistical theory of gases). Entirely new effects, such as the EMC effect $[0]$, can be discovered and yield new physical insight. And perhaps most importantly, nuclear reinteractions serve as an analyser of the newly produced hadronic state, and as such can provide important information on the hadronization mechanism, one of the central unsolved problems of modern day physics.

Prior investigations have focused primarily on comparing properties of different nuclei in high energy scattering ${ }^{|10|}$. This investigation uses a different tack. Data for the same nucleus ( $\mathrm{Ne}$ ) are compared under identical circumstances but for four different types of incident beam: $\pi^{+}, \pi^{-}, \nu$, and $\nu$. Nuclear effects of neutrinos have not been extensively investigated.

Having both particle and antiparticle beams permits us to compare isospin-rotated final states (the Ne nucleus is isospin 0 ), and thus to identify additional baryons in the final state where they cannot be identifled by ionisation alone (due to high momentum ${ }^{[11]}$ ). Differences in the average path length traversed by a $\pi$-induced system and $\mathbf{a}(\bar{\nu})$-induced system provide a link to the number of reinteractions ${ }^{[12]}$. Further nuclear rein- teraction differences may arise from the four-jet pion system and two-jet neutrino system, or the different initial quark content of the system ${ }^{[12]}$.

What tools are available in the final state to study nuclear reinteractions? Clearly protons in the final state are directly linked to nuclear reinteractions, and both protons identified by ionization and the isospin technique are extensively studied and used in the analysis. Nuclear reinteractions will increase the net multiplicity, and many multiplicity features are studied and compared for leptonic and hadronic systems in our experiment. Inclusive distributions, normalized as $\left(1 / \sigma_{r}\right) d \sigma_{r} / d \theta$, which represent the average multiplicity for reaction-type $\sigma$, per $d \theta$ interval, are analyzed and compared. Correlations of the lorents invariants of the interaction (such as $x_{B J}$ and $W^{2}$ ) are considered. Two-particle rapidity correlations are analyzed. A search for coherent events is carried out, and a search for a resonance signal of proton production $\left(\Delta_{\text {faet }}^{++}\right)$. An algorithm (called the NUCTST algorithm) has been developed to divide events into nuclear-enriched and nuclear-depleted categories, and is often used to analyze our data.

Briefly, the experimental technique used was as follows.

The neutrino experiment took place at Fermilab, near Batavia, III., USA. High energy pions and kans focused by a quadrupole train generated the neutrino beam used from their decays to lepton and neutrino. Interactions on neon were analyzed in the Fermilab 15 foot bubble chamber. The muon produced in charged current interactions was identifled by the External Muon Identifier ${ }^{\mid 13]}$, an array of proportional wire chambers separated from the chamber by absorber, by extrapolating leaving tracks from the bubble chamber to the EMI. Interactions with $a$ 
muon meeting cut criteria were then fully measured on precision digitization measuring devices, including all neutrals within two radiation lengths. The results of these measurements form the neutrino data sample used in this experiment.

The pion experiment took place at CERN, near Geneva, Switzerland Pions were generated from $200 \mathrm{GeV} / \mathrm{c}$ protons incident on a beryllium target, and $\pi^{+}$or $\pi^{-}$beams were selected at $30 \mathrm{GeV} / \mathrm{c}$ or $64 \mathrm{GeV} / \mathrm{c}$ Interactions on neon were analy zed in the BEBC bubble chamber, with the beam line tuned to inject only a few pions per chamber cycle. The pictures obtained were fully scanned. Interactions occuring on accepted beam tracks had their charge multiplicity, both positive and negative, recorded, and the multiplicity of identifled proton tracks recorded. The results were corrected for $\mathrm{H}_{2}$ interactions, coherent interactions, and had a multiplicity cut applied to eliminate scan inefficiencies. In addition a sample of events for $\pi^{+}$and $\pi^{-}$incident at $30 \mathrm{GeV} / \mathrm{c}$ had all charged tracks measured. The multiplicity scan results and the measurement sample form the pion data sample used in this experiment.

Finally, I will briefly describe the physical layout of this dissertation.

Section 1.2 is a glossary of symbols used throughout the remainder of the dissertation. Chapter 2 provides theoretical background for the analysis, including a cast of characters (elementary particles), a script for their interaction (the ElectroWeak force, the Strong force), a scenario (high energy neutrino interactions), and finally a brief excursion into nuclear reinteraction effects theories. Chapter 3 describes in detail our neutrino experiment, and chapter 4 details our pion experiment. Chapter 5 deals with a few key analysis techniques used, including the NUCTST algorithm. Chapters 8, 7, and 8 present the results of the analysis, grouped respectively into multiplicity results, kinematic distribution results, and whatever might not fit into the first two categories ("other" results).

For a brief summary of the results, see sections 6.1, 7.1, and 8.1. These sections, titled "Chapter Overview", provide highlights of the results contained in each respective chapter.

\subsection{Some frequently used symbols.}

The following is a glossary of symbols used in this dissertation. A few symbols, by common usage, have more than one meaning, in which case it is usually clear from the context which meaning the symbol carries. If doubt could remain after considering the context of usage, the symbol meaning is explicitly given where it appesrs in the text.

The ordering is alphabetical. Greek symbols are alphabetized by their english transcription. Generally there is a reference (enclosed in square brackets) to the section where the symbol is defined or used following the brief description given here.

$$
\sim \text { Glossary }
$$

A number of nucleons in a nucleus

$A_{\mu} \quad$ gauge fields or photon field [2.2]

b baryon

b impact parameter [appendix A]

$\beta \quad$ velocity in units where $c=1$ 
c speed of light in racuum

$c_{0} \quad$ speed of sound in nuclear matter [2.5]

$C_{p r}^{k-} \quad$ the normalized absolute moment in $N_{p r}^{-}$: $300 \mathrm{pt}\left\langle\left(N_{\mathrm{pr}}^{-}\right)^{k}\right\rangle /\left\langle N_{\mathrm{pr}}^{-}\right\rangle^{k}[8.2]$

CC neutrino Charged Current event

d diquark

$\Delta_{\text {faet }}^{++} \quad \Delta^{++} \rightarrow p+\pi$ with the proton not identifled by ionization [8.4]

$\delta_{\alpha \beta} \quad$ Kroneker delta

$\delta_{G} \quad$ the variation under a group symmetry G [2.2]

$D_{p r}^{-} \quad$ the dispersion in $N_{p r}^{-}: \sqrt{\left\langle\left(N_{p r}^{-}\right)^{2}\right\rangle-\left\langle N_{p r}^{-}\right\rangle^{2}}$ [B.2]

$D_{\mu} \quad$ covariant derivative $\theta_{\mu}-i g A_{\mu}[2.2]$

e electron charge or electron

E energy

$\epsilon_{\alpha \beta \ldots} \quad$ completely antisymmetric tensor with values 0 or 1 (LeviCivita symbol)

$E_{\nu} \quad$ energy of incident neutrino [2.4]

$f_{p r}^{2--} \quad$ Mueller's correlation function in $N_{p r}^{-}$: $\left.\left\langle N_{p r}^{-}(a) N_{p r}^{-}(b)\right\rangle-\left\langle N_{p r}^{-}(a)\right\rangle\left\langle N_{p r}^{-}(b)\right\rangle\right\}$ for $a \neq b[$ b.6]

$f_{p r}^{8---} \quad$ correlation function in $N_{p r}^{-}:\left\langle\left(N_{p r}^{-}-\left\langle N_{p r}^{-}\right\rangle\right)^{8}\right\rangle+2\left\langle N_{p r}^{-}\right\rangle-$ $3\left(D_{\mathrm{pr}}^{-}\right)^{2}[6.6]$

$\int_{\text {coh }} \quad$ fraction of all events that are coherent events [4.2]

$f^{\text {ruc }}$ ratio of nuclear to (nonnuclear + nuclear) events: $N^{N u c t s t-+2} /\left(N^{N u c t s t-+2}+N^{N u c t s t--2}\right)[7.2]$
Soiop fraction of all protons that can be identiffed by ionization [4.2]

$F_{i} \quad$ weak structure function $[2.4]$

$F_{\mu \nu} \quad$ the $U(1)$ gauge invariant field combination $\partial_{\mu} A_{\nu}-\partial_{\nu} A_{\mu}$ [2.2]

$F_{\mu \nu}^{i} \quad$ the extension of $F_{\mu \nu}$ to a non-abelian group [2.2]

$g \quad$ coupling constant $[2.2]$

G Fermi coupling constant

$G_{B o n n}$ energy correction scale factor for Bonn neutrino energy correction method [3.2]

$G_{p T b a l}$ energy correction scale factor for $p_{T}^{\text {bal }}$ neutrino energy correction method [3.2]

lorentz factor $1 / \sqrt{1-\beta^{2}}$

$\gamma_{\mu} \quad$ 4-dimensional Dirac $\gamma$ matrices (see appendix B)

$\gamma_{5}$ the product $\gamma_{1} \gamma_{2} \gamma_{8} \gamma_{4}$

$\gamma_{p r}^{-} \quad$ skewness in $N_{p r}^{-}:\left\langle\left(N_{p r}^{-}-\left\langle N_{p r}^{-}\right\rangle\right)^{8}\right\rangle /\left(D_{p r}^{-}\right)^{8}[$ [B.6]

$\overrightarrow{\mathbf{H}}$ or $\boldsymbol{H}^{i} \quad$ the generators of a compact continuous group [2.2]

$j_{\lambda}^{+}, j_{\lambda}^{-}, j_{\lambda}^{0} \quad Q_{\text {finat }}-Q_{\text {initial }}=+1,-1,0$ weak leptonic currents $[2.2]$

$J_{\lambda}^{+}, J_{\lambda}^{-}, J_{\lambda}^{0}$ weak hadronic currents $[2.2]$

$J^{P} \quad$ (spin) $^{\text {parity }}[2.3]$

$\boldsymbol{k}_{\boldsymbol{F}} \quad$ Fermi momentum [7.3]

$\mathcal{L} \quad$ lagrangian density $[2.2]$

M matrix element [2.2] 


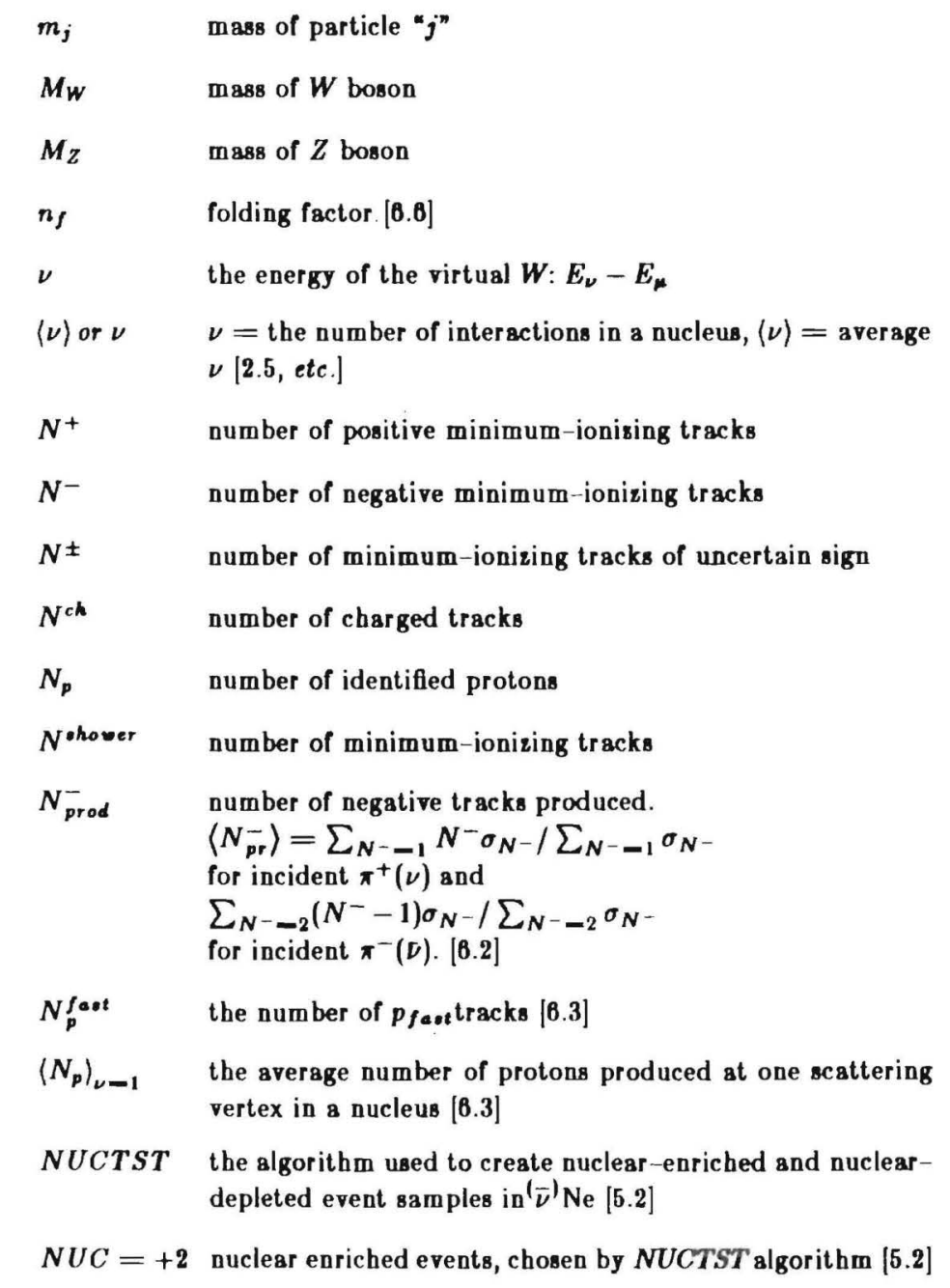

$N U C=0 \quad$ all events $[5.2]$

$N U C=-2$ depleted events, chosen by NUCTST algorithm [5.2]

$p \quad$ four-momentum or magnitude of $\overrightarrow{\mathbf{p}}$

$\overrightarrow{\mathbf{p}} \quad$ three momentum

$\psi \quad$ fermion field

$\psi(z) \quad$ KNO scaling function [6.5]

$p_{\text {fast }}$ a non-identified proton, due to $p_{\text {prot }}>800 \mathrm{MeV} / \mathrm{c}$ [7.5]

$p_{T} \quad$ transverse momentum (to incident beam)

$P(i) \quad$ the probability of condition " $i$ "

- $\Pi(\nu) \quad$ the probability of $\nu$ interactions in a nucleus [6.3]

$q_{m}(x)$

$Q$

$Q^{2}$

$r$

$\overrightarrow{\mathbf{F}}$

r Ne radius of Neon

$R \quad$ the ratio $\sigma\left(e^{+} e^{-} \rightarrow\right.$ hadrons $) / \sigma\left(e^{+} e^{-} \rightarrow \mu^{+} \mu^{-}\right)[2.3]$

$R_{A}$ the ratio of multiplicity in $X+N$ ucleus $(A)$ to multiplicity in $X+N$ ucleon $[$ 6.4] 
$\tilde{R}_{A}^{(2)} \quad R_{A}$ evaluated in our neutrino data using the NUCTST algorithm

$R_{D} \quad$ ratio of production of baryon decuplet states to baryon octet states [8.4]

$R_{\Delta} \quad$ the efficiency of a $\Delta$ cut $[8.4]$

$R\left(y_{1}, y_{2}\right)$ the normalized two-particle rapidity correlation function [8.2]

- the square of the hadronic four-momenturn in hadronic scattering, ie $(\pi+p)^{2}$

$\sigma \quad$ cross section

$t$ the square of the four-momentum transfer between incident current and target in a diffractive process [8.3]

$\tilde{t}$ the four-momentum transfer between incident current and target in a diffractive process $[8.3]$

$t_{\min } \quad$ the minimum possible value of $|t|$, given $M_{X}$, incident current $\overrightarrow{\mathbf{q}}$, and magnitude of the momentum of the $X$ system [8.3]

for $r^{x}, r^{y}, r^{*}$ Pauli matrices (see appendix B)

$\theta_{C} \quad$ Cabbibo mixing angle between quark generations [2.3]

$\theta_{W} \quad$ Weak mixing angle between $S U(2)$ and $U(1)$ coupling constants [2.2]

$W^{2} \quad$ the square of the hadronic four-momentum in hadronic scattering, ie $(q+p)^{2}$

$x_{ \pm}$

$t \pm z$, light cone variables $[2.5$

$x_{B J}$ or
Feynman $x, p_{\|} / p_{\max }$, where $p_{\max }$ is the maximum possible momentum of a particle

leptonic variable, $y=q \cdot p / k \cdot p[2.4]$

rapidity, $y=\frac{1}{2} \ln \left[\left(E+p_{\|}\right) /\left(E-p_{\|}\right)\right]$, with $p_{\boldsymbol{\|}}$ along incident beam direction

rapidity in lab frame

rapidity in CM frame

$E / E_{m a x}$, the fraction of maximum possible energy a particle carries

$z$ in the lab frame

$z$ in the CM frame

atomic number 


\section{Notes for Chapter 1}

1. see, for example, A. deShalit and H. Feshbach, "Theoretical Nuclear Physics", John Wiley and Sons (New York, 1974)

2. see, for example, A. B. Migdal, Rev. Mod. Phys. 50,107 (1978)

3. the EMC effect was first noted by the European Muon Collaboration in J.J. Aubert et al., CER N-EP/83-14 (1983), and rapidly confirmed by SLAC experiments (A. Bodek et al., "Electron Scattering from Nuclear Targets and Quark Distributions in Nuclei", SLAC-PUB3041 (1983))

4. for extensive measurements of the EMC effect, see R.G. Arnold et al., "Measurements of the A-Dependence of Deep-Inelastic Electron Scattering from Nuclei", SLAC-PUB-3257 (1983)

5. A. Bodek and J.L. Ritchie, Phys. Rev. D23, 1070 (1981); Phys. Rev. D24, 1400 (1981)

b. C.H. Llewellyn Smith, Phys. Lett. 128B, 107 (1983); M. Ericson and A.W. Thomas, Phys. Lett. 128B, 112 (1983)

7. E.L. Berger, F. Coester, and R.B. Wiringa, Phys. Rev. D29, 398 $(1984$

8. J.J. Aubert et al., Phys. Lett. 105B, 315, 322 (1981)

9. $\quad$ see references 3 and 4

10. see, for example, W. Busza et al., Phys. Rev. Lett. 34, 836 (1975); J.E. Elias et al., Phys. Rev. D22, 13 (1980)

11. for a discussion, see section 6.3B and section 7.5.

12. see section 2.5 for a discussion
13. see J. Orthel, PhD Thesis, University of California at Berkeley (1979) 


\section{CHAPTER ?}

\section{THEORETICAL BACKGROUND}

See Appendix B for the notational convention used in this chapter.

\subsection{Elementary particles.}

Since Democritus ${ }^{[1]}$ first intuited that there must be some "smallest" particle of matter, the "atom", the nature of such "elementary particles" has been a subject of theoretical and experimental inquiry. One of the requirements we might posit for an "elementary" particle is that there should be only a few types of such elementary particles, from which we could build an arbitrarily complicated reality by using a specifled iteration scheme, or "theory".

On this basis of number of types, science has been a downhill inquiry since the days of Aristotle, who (unfortunately inaccurately) managed to reduce all matter to only four basic constituents: Earth, Air, Fire, and
Water. With the discovery of elements, or in modern parlance atoms, and the enumeration of the periodic table ${ }^{|2|}$ the number of basic constituents grew to the size of the periodic table, or something the order of 100 , if we don't count isotopes as anything new. But then a great leap forward (or downward, as the case may be, to a smaller number of "elementary" particles) occured with the discovery of the electron and the elucidation of the structure of the atom as a lump of heavy positive charge surrounded by orbiting electrons ${ }^{|a|}$. Since atoms are electrically neutral, and all electrons have the same charge, the implication follows that the positive charge in the atomic nucleus also must come in lumps, with some neutral lumps thrown in to account for atomic isotopes.

Calling the positive lumps "protons" and the neutral lumps "neutrons", science seemed to have at last caught up with Aristotle and reduced all of nature of four constituents: Electrons, protons, neutrons, and something to carry light around (either an sther or particles of light, "photons"). Unfortunately the ability to probe distance scales on the order of atomic distances and smaller, while providing an initial simpliflcation, proved a mixed blessing and soon the "elementary particle" cat was again out of the bag. Heavy leptons, or muons, which were exactly like electrons except for some added weight, were discovered ${ }^{|4|}$. Pions, lightweight spin-0 particles which act as an effective carrier of the strong force to bind together the positive charge in an atomic nucleus, turned up ${ }^{|5|}$. Neutral leptons, or neutrinos, were found in varieties to match both electrons and muons ${ }^{101}$.

A veritable plethora of particles associated with distance scales $\leq 1$ $\mathrm{fm}$ came popping out of nucleons as soon as energies appropriste for this distance scale became available ${ }^{[7]}$. All together, the elementary particle 
candy shop was again offering on the order of 100 different varieties. Such a complicated scenario veritably cried out for a further simplification analagous to the periodic table: Finding a few constituents from which the complicated list of particles could be built by following some simple rules.

Leptons, which we can define as fermions that do not feel the strong force, were poor candidates for having constituents. Tests related to the theory of the electromagnetic force (Quantum ElectroDynamics, or QED ${ }^{[8]}$ ) established that electrons had to be structureless, pointlike particles, down to distances of $10^{-3} \mathrm{fm}$, or very much smaller than nucleons ${ }^{|0|}$. However the puffy nucleons and their associated grab bag of particles were ideal candidates for having constituents. In a manner exactly equivalent to Rutherford's original experiments finding the heavy nucleus in the atom ${ }^{[10]}$, constituents of the strong-interaction mesons and baryons were discovered ${ }^{[11]}$ and their nature slowly ferreted out.

These constituents, quirkily called "quarks" ${ }^{[12 \mid}$ from a quote in James Joyce's Finnegans Wake ("three quarks for Muster Mark!", with the obscure connection being the "three"), could be used to build up all the strong-interaction particles according to a set of combinatorial rules called $S U(3)$. Again the number of elementary particles contracted dramatically, down to 2 leptons and their neutrinos in the lepton sector and 3 quarks in the hadronic sector, for a total of 7 . (Here we are counting antiparticles as being part of the "rules" of making particles, and we have magically explained away light as merely a symmetry transformation property of the electromagnetic theory, analagously to gravitation in General Relativity. $\left.{ }^{\mid[3 \mid}\right)$
However, the story is not yet closed. As science probed to ever-smaller distance scales, or equivalently ever-higher masses, new particles on the lowest constituent level currently known were discovered. The three old quarks, called $u, d$, and $s$, for up, down, and strange, were joined by $c$ and $b$, for charm ${ }^{|14|}$ and bottom ${ }^{|15|}$, and a new even heavier lepton called $r$ (which presumably has an associated neutrino, $\nu_{r}$ ) was discovered in $e^{+} e^{-}$ annihilation experiments ${ }^{[10]}$

To round out this cast of characters there are 12 "gauge fields", 80 called because they appear as manifestations of a symmetry property of the theory, just as gravitation appears as a manifestation of General Coordinate lnvariance. Of these only four have been observed directly, the photon $\left(A_{\mu}\right)$, the two $\Delta Q=1$ weak interaction flelds $\left(W^{ \pm}\right)$, and the $\Delta Q=0$ weak interaction field $\left(Z^{0}\right)$, which together are the carriers of the electroweak force ${ }^{[17]}$. The strong force carriers make up the remaining 8 gauge flelds, called gluons.

Due to the regular arrangement of particles under the rules of the electroweak interaction (see Section 2.2), a sixth quark, the $t$ (for top) quark is postulated and believed to exist. In addition, the procedure for giving the weak interaction gauge flelds a mass so that the weak interaction will be short range leaves behind an artifact, at least one spin0 field called a Higgs boson ${ }^{|18|}$. Currently no experimental indication of a Higgs boson has been found.

This leaves two questions in the list of truly "elementary" particles.

First, no quark has ever been observed in a free state, raising questions about the validity of having an "elementary parucle" that cannot be directly observed, even though $u$ and $d$ quarks can essentially be treated 
as massless objects. As discussed in section 2.3 , the strong interaction may require a type of inverse vacuum screening, which in turn would imply that quarks always come in the multiquark combinations, such as $q \bar{q}$ or $q q q$. That is, quarks are "conflned" inside hadrons, or multi-quark objects, making the question of direct observation moot. This conflnement would account for lack of observation of free quarks.

Secondly, will the list of quark-and lepton-level elementary particles continue to fll out indefinitely as prior "elementary particle" lists have? Two reassurances that this may indeed be the bottom level are at hand One is that a cosmological argument ${ }^{[10]}$ puts the maximum number of light neutrinos at 4 , implying a maximum of one more lepton and quark generation. (In any case, a forthcoming measurement of the $Z^{\mathbf{0}}$ decay rate will determine the number of light neutrinos exactly $\left.{ }^{[10]}\right)$. The second is that strong interaction theory establishes a limit of 18 types of light quarks in order to retain an observed property of the theory known as "asymptotic freedom" ${ }^{[20]}$.

The current list of elementary particles, including the projected $t$ quark and the artifact Higgs field $h$, is shown in figure 2.1.1. The quarks and leptons are arranged in left-handed doublets and right-handed singlets, as is appropriate for the $S U(2)_{L} \otimes U(1)_{E M}$ electroweak theory. Since the masses of the quarks are not eigenstates of the electroweak interaction hamiltonian, the quark fields appearing in the doublets are actually mixtures, with Cabbibo mixing $\left.{ }^{\mid 21}\right|_{\text {shown }}$ on the figure (see Section 2.2).

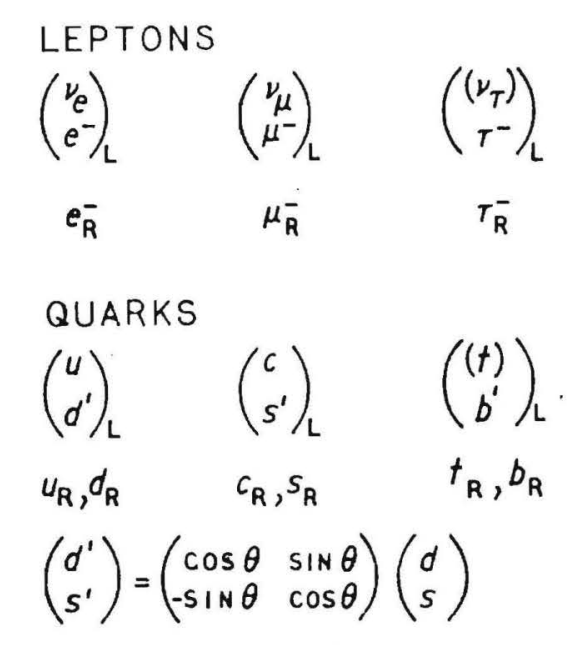
GAUGE FIELDS
ELECTROWEAK: ح
$A_{\mu}, W_{\mu}^{ \pm}, z_{\mu}^{\circ}$
STRONG : eeceeleee
$q_{\mu}^{i}, i=1 \ldots 8$

HIGGS

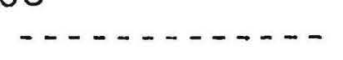

TIGURE 8.1.1.

Elementary particles. (Arranged according to $S U(2)_{L} \otimes U(1)_{E M}$, with Cabbibo mixing.) 


\section{2.2. The ElectroWeak Interaction.}

The electroweak interaction ${ }^{[22\}}$ is described by a unified, renormalizable quantum theory of the electromagnetic and weak interactions due to S. Weinberg ${ }^{[28 \mid}$ and A. Salam ${ }^{[24]}$, who developed the theory independently. This theory is the simplest realistic model that can be devised to unify the weak and electromagnetic interactions using the principle of gauge invariance, and is termed the "standard model." As of this writing, the standard model is in agreement with all measurements testing it ${ }^{[25]}$, including the famous prediction of the masses of the $W^{ \pm}$and $Z$ gauge bosons, recently observed for the first time at the CERN $p \bar{p}$ colider ${ }^{[26]}$.

Quarks couple to all known interactions, whereas leptons do not couple to the strong interaction; that is, leptons do not carry a "strong" charge. Since neutrinos are electrically neutral, neutrinos cannot couple to the maguetic interaction and couple only to the weak interaction (gravitation is ignored in this discussion). Neutrinos are thus ideally suited for isolating weak interaction effects.

At low momentum transfers $\left(|q|<M_{W}\right.$, where $q$ is the four momentum transfer $\boldsymbol{k}-\boldsymbol{k}^{\prime}$ and $\boldsymbol{M}_{\boldsymbol{W}}$ is the mass of the $\boldsymbol{W}$ boson, which mediates the weak interaction), the weak interaction is well described by a local current $x$ current form, where the currents are evaluated at the same space-time point, as opposed to the electromagnetic interaction. This phenomenological form is a result of the large mass of the $W$ boson, with the CERN values for $M_{W} \approx 81 \mathrm{GeV} / \mathrm{c}^{2}$ and $M_{Z} \approx 93 \mathrm{GeV} / \mathrm{c}^{2}$ Fermi first postulated such a current $X$ eurrent interaction in $1934^{|27|}$ to provide a model for nuclear $\beta$-decay. The first-order low energy limit of the standard model reduces to the current $X$ eurrent form and the usual electromagnetic lagrangian.

"Gauge theories" are so called because a symmetry of the lagrangian provides an invariance under some set of transformations; if these transformations are connected to a space-time symmetry, they provide a "gauge". However, there is no reason to restrict this symmetry to such geometrical gauges, and the weak interaction gauge symmetry is actually an isospin symmetry coupling to weak isopin charges of quarks and leptons. Such a symmetry not connected with space-time coordinates is referred to as an "internal" symmetry of the interacting field.

To derive the rules of calculation of a model we demand that the action be stable under arbitrary variations, i.e. that

$$
\delta \int_{t_{1}}^{t_{3}} \mathcal{L}\left(\phi_{i}, \partial_{\mu} \phi_{i}\right) d^{3} r d t=0
$$

for arbitrary $t_{1}$ and $t_{2}$ and where the variation vanishes at $t_{1}$ and $t_{2}$. L is the lagrangian density and is a function of a set of fields $\phi_{i}, i=1 \ldots N$, and their gradients. The lagrangian must be a Lorente scalar to yield a Lorents invariant theory. The lagrangian that yields the Dirac equation of motion for a free spin-1/2 fermion field $\psi$ is

$$
\mathcal{L}=-\psi^{\dagger} \gamma_{4}\left(\gamma_{\mu} \partial_{\mu}+m\right) \psi,
$$

where the $\gamma_{\mu}, \mu=1 \ldots 4$, are the usual 4-dimensional Dirac gamma matrices and $m$ is the mass of the field.

Any even power of a feld, such as the mass term in equation 2.8.1, is invariant under a unitary transformation of the field

$$
\psi \rightarrow \psi^{\prime}=u \psi
$$


since $\psi^{\dagger} \psi \rightarrow \psi^{\dagger} u^{\dagger} u \psi=\psi^{\dagger} \phi$. This suggests that the full lagrangian may be invariant under such a transformation, as indeed it is if $u \neq u(x)$, that is if the transformation does not depend on the space-time coordinates. Such a transformation is called a global transformation since the field $\psi$ is rotated by the same amount throughout space-time. If, however, we require the lagrangian to have this symmetry at each point of space-time independently, then $u=u(x)$, and terms involving the derivative of the field gain an added term:

$$
\partial_{\mu} \psi \rightarrow \partial_{\mu} \psi^{\prime}=u(x) \partial_{\mu} \psi+\left[\partial_{\mu} u(x)\right] \psi
$$

(This more stringent requirement of independence of $z$ results in "local" symmetry)

Any symmetry has associated with it a group $G$ and a group operation '*), since a symmetry literally means that there is a set of elements that map into themselves under some iterated operation: $S \rightarrow S^{\prime}=S$. The added term in 2.2.3 causes the lagrangian to lose its group symmetry for a local transformation: $\delta_{G} \mathcal{L} \neq 0$. To restore this symmetry we add a set of new fields to compensate for the derivative term:

$$
\partial_{\mu} \psi \rightarrow \partial_{\mu} \psi-i g A_{\mu} \psi \equiv D_{\mu} \psi
$$

and require the $A_{\mu}$ to transform in such a way as to cancel the added term in 2.2.3. Terms involving $D_{\mu}$ will then transform like $\psi$, ie $\delta_{G} D_{\mu} \psi=$ 0 . These added fields are called gauge fields, and since they have the same transformation properties as $\partial_{\mu}$, are always vector flelds. $D_{\mu}$ is the "covariant" derivative.
Any unitary transformation $u$ can always be written as $e^{-i H}$ with $H$ hermitian; for the subcase of a continuous compact group we write $u=$ $e^{-i \vec{\theta}(x) \overrightarrow{\mathbf{H}}}$, where $\overrightarrow{\mathbf{H}}$ spans the group space and $\vec{\theta}(x)$ therefore represents the parameter space of the group. If choose $G=U(1)$ (unitary 1dimensional), then $\overrightarrow{\mathbf{H}}=q$, a real constant. In this case we require

$$
D_{\mu}^{\prime} \psi^{\prime}=u D_{\mu} \psi
$$

or

$$
\left(\partial_{\mu}-i g A_{\mu}^{\prime}\right) e^{-i q \theta(x)} \psi=e^{-i q \theta(s)}\left(\partial_{\mu}-i g A_{\mu}\right) \phi
$$

which implies

$$
A_{\mu}^{\prime}=A_{\mu}-i \frac{q}{g} \partial_{\mu} \theta(x)
$$

that is,

$$
\delta_{U(1)} A_{\mu}=-i \stackrel{q}{g} \partial_{\mu} \theta
$$

Since the new fields $A_{\mu}$ contribute to the stress-energy of the system, they must appear in $\mathcal{L}$ not coupled to in some term $F\left(A_{\mu}\right)$, where $F\left(A_{\mu}\right)$ must satisfy $\delta_{U(1)} F\left(A_{\mu}\right)=0$ to retain gauge invariance. Since $\delta_{U(1)} A_{\mu} \sim \partial_{\mu} \theta$, the obvious solution is to create an operator antisymmetic in derivatives to eliminate the $\theta$ dependence: $F_{\mu \nu} \equiv \partial_{\mu} A_{\nu}-\partial_{\nu} A_{\mu}$. Then $F_{\mu \nu} F^{\mu \nu}$ will satisfy the requirement of $\theta$ invariance and Lorentz invariance.

We have arrived at the full $U(1)$ lagrangian:

$$
L^{B M}=-\frac{1}{4} F_{\mu \nu} F^{\mu \nu}-\psi^{\dagger} \gamma_{4}\left(\gamma_{\mu} D_{\mu}+m\right) \phi
$$

Lagrangians for more complicated symmetry groups are constructed in analagous fashion. 


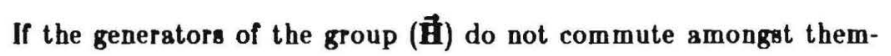
selves, then by the group composition rule $\left[H^{i}, H^{j}\right]=i c_{i j k} H^{k}$, where the $c_{i j k}$ are the structure constants of the group. For each generator we introduce a gauge field $A_{\mu}^{i}$ to compensate for the derivative term in 2.2.3, and $D_{\mu}$ in 2.2 .4 becomes $D_{\mu}=\left(\partial_{\mu}-i g \overrightarrow{\mathbf{A}}_{\mu} \cdot \overrightarrow{\mathbf{H}}\right)$. Then as before condition 2.2.5 requires

$$
\overrightarrow{\mathbf{A}}_{\mu}^{\prime} \cdot \mathbf{H}=u(\theta) \mathbb{\mathbf { A }}_{\mu} \cdot \mathbf{A} u^{-1}(\theta)-\frac{i}{g}\left(\partial_{\mu} u(\theta)\right) u^{-1}(\theta)
$$

For an inflinitesimal transformation $\left(\theta^{i}(x) \rightarrow \epsilon^{i}(x)\right.$, so $\exp (-i \vec{\theta} \cdot \overrightarrow{\mathbf{H}}) \rightarrow$ $1-\overrightarrow{i \epsilon} \cdot \overrightarrow{\mathbf{H}})$ this becomes

$$
\vec{A}_{\mu}^{\prime} \cdot \overrightarrow{\mathbf{H}}=(1-i \vec{i} \cdot \overrightarrow{\mathbf{H}}) \vec{A}_{\mu} \cdot \overrightarrow{\mathbf{H}}(1+i \vec{i} \cdot \overrightarrow{\mathbf{H}})-\frac{i}{g}\left(-i\left(\partial_{\mu} \vec{z}\right) \cdot \overrightarrow{\mathbf{H}}\right)(1+i \vec{z} \cdot \overrightarrow{\mathbf{H}}),
$$

or

$$
\overrightarrow{\mathbf{H}} \cdot \delta \overrightarrow{\mathbf{A}}_{\mu}=-i(\vec{\epsilon} \cdot \overrightarrow{\mathbf{H}})\left(\overrightarrow{\mathbf{A}}_{\mu} \cdot \overrightarrow{\mathbf{H}}\right)+i\left(\overrightarrow{\mathbf{A}}_{\mu} \cdot \overrightarrow{\mathbf{H}}\right)(\vec{t} \cdot \overrightarrow{\mathbf{H}})-\frac{1}{g}\left(\partial_{\mu} \vec{t}\right) \cdot \overrightarrow{\mathbf{H}}
$$

to first order in $\vec{z}$. The first two terms in $\mathbf{2 . 2} .12$ constitute the comutater

$$
-i \epsilon^{i} A_{\mu}^{j}\left[H^{i}, H^{j}\right]=\epsilon^{i} A_{\mu}^{j} c_{i j k} H^{k}
$$

where we have written out the vectors in component notation and use the convention that repeated indices are summed over. Since the $H^{i}$ are linearly independent, this gives the variation in $A_{\mu}^{i}$

$$
\delta A_{\mu}^{i}=-\frac{1}{g} \partial_{\mu} \epsilon^{i}+c_{i j k} \epsilon^{j} A_{\mu}^{k} .
$$

As seen from the derivation, the new term $\sim A_{\mu}$ comes from the noncommuting property of the $\boldsymbol{H}^{i}$. In turn this new term implies that the simple form used for $F_{\mu \nu}$ in 2.2 .9 is no longer gauge invariant. If we can find a modified $F_{\mu \nu}^{i}$ that transforms like the fundamental representation of $\mathrm{G}$, then the combination $F_{\mu \nu}^{i} F^{i \mu \nu}$ will satisfy $\delta_{G} F_{\mu \nu}^{i} F^{i \mu \nu}=0$ by the antisymmetry of the structure constants. From the way in which $A_{\mu}^{i}$ transforms (2.2.14), we see that the terms from $\partial_{\mu} A_{\nu}-\partial_{\nu} A_{\mu}$ that are $\sim\left(\partial_{\mu} \epsilon\right) A$ can be cancelled by adding terms to $F_{\mu \nu}^{i}$ that are of the form $A^{j} A^{k}$, since $\delta A^{i} \sim \partial_{\mu} \epsilon^{i}$. If we add $g c_{i j k} A_{\mu}^{j} A_{\nu}^{k}$ to $\partial_{\mu} A_{\nu}-\partial_{\nu} A_{\mu}$, this exactly cancels all terms $\sim\left(\partial_{\mu} \epsilon\right) A$ and leaves $\partial_{\mu} A_{\nu}-\partial_{n} u A_{\mu}+g c_{i j k} \epsilon^{i} A_{\mu}^{j} A_{\nu}^{k} \sim$ $F_{\mu \nu}$ and therefore this new combination transforms in the required way. So defining

$$
F_{\mu \nu}^{i}=\partial_{\mu} A_{\nu}^{i}-\partial_{\nu} A_{\mu}^{i}+g c_{i j k} A_{\mu}^{j} A_{\nu}^{k}
$$

gives

$$
\delta_{G} F_{\mu \nu}^{i}=e_{i j k} \theta^{j} F_{\mu \nu}^{k}
$$

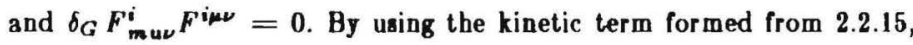
$-\frac{1}{4} F_{\mu \nu}^{i} F^{i \mu \nu}$, and the appropriate covariant derivitive for $\mathrm{G}$

$$
D_{\mu}=\partial_{\mu}-i g \overrightarrow{\mathbf{A}}_{\mu} \cdot \overrightarrow{\mathbf{H}}
$$

we can form a lagrangian such that $\delta_{G} \mathcal{L}=0$ which will have $n$ gauge fields in it, where $\boldsymbol{n}$ is the dimension of $\mathbf{\mathbf { H }}$, or equivalently the fundamental representation of $\mathrm{G}$

This provides the key to unifying the electromagnetic and weak interactions. Since there exist $\Delta Q= \pm 1$ (for example, $\nu_{\mu}+p \rightarrow \mu^{-}+\Delta^{++}$ and $\nu_{\mu}+p \rightarrow \mu^{+}+n$ ) and $\Delta Q=0$ (for example, $\nu_{\mu}+p \rightarrow \nu_{\mu}+p$ ) weak interaction currents and no other weak currents, $n_{\text {wesk }}=3$. The simplest continuous compact unitary group that can have dimension 3 
is $S U(2)(S U(n)$ is the group of $n \times n$ complex unitary matrices with determinant $=+1$, which has $2 n^{2}-n^{2}-1=n^{2}-1$ real parameters). As we have seen electromagnetism is the result of a $U(1)$ symmetric theory. This suggests the direct product $S U(2) \otimes U(1)$ as the symmetry group of the weak interaction, which since it is a direct product can have two independent coupling constants $g$ and $g^{\prime}$, a combination of which will be the electric charge $\alpha=1 / 137$ (in units of $\hbar=c=1$ ).

An immediate problem is that any term of the form $m\left(A^{i}\right)^{2}$ is not gauge invariant, as can be seen from 2.2.14, so all gauge fields are massless. This is fine for the photon, which is indeed massless, but as we have discussed the weak gauge bosons must be massive. This problem is solved by noting that to determine the mass of a field $\phi$, we expand around the ground state of $\phi, \min (\phi)$, or $(\phi)_{V A C}$ (i.e. the expectation value of $\phi$ in the vacuum). Generally $\langle\phi\rangle_{V A C}=0$, but if $\langle\phi\rangle_{V A C} \neq 0$, then the normal modes will be shifted and it may be necessary, depending on $\mathcal{L}$, to consider different combinations of fields as the normal modes. This can result in what we might term an "effective mass" for a fleld. This technique of adding an additional spin-0 field to the lagrangian which acquires a vacuum expectation value, and then through its coupling via $D_{\mu}$ gives a mass to some subset of the gauge bosons, is called the Higgs mechanism

The phenomenological current $\times$ eurrent form of the weak lagrangian for purely leptonic processes is

$$
L_{\text {lep }}^{W}=\frac{G}{\sqrt{2}}\left(j_{\lambda}^{+} j_{\lambda}^{-}+\frac{1}{2} j_{\lambda}^{0} j_{\lambda}^{0}\right)
$$

where

$$
\begin{aligned}
& j_{\lambda}^{-}=i \sum_{1}^{1} \psi_{i}^{\dagger} \gamma_{1} \gamma_{\lambda}\left(1+\gamma_{5}\right) \psi_{\nu_{1}} \\
& j_{\lambda}^{+}=i \sum_{l}^{1} \psi_{\nu_{1}}^{\dagger} \gamma_{4} \gamma_{\lambda}\left(1+\gamma_{6}\right) \psi_{l} \\
& j_{\lambda}^{0}=i \sum_{l}^{1}\left[\psi_{\nu_{1}}^{\dagger} \gamma_{4} g_{\nu} \gamma_{\lambda}\left(1+\gamma_{5}\right) \psi_{\nu_{l}}+\phi_{1}^{\dagger} \gamma_{4} \gamma_{\lambda}\left(g_{V}+g_{A} \gamma_{5}\right) \psi_{l}\right],
\end{aligned}
$$

as is well-determined from muon decay and neutral-current neutrino scattering. (The superscript refers to the charge in charge $Q_{f \text { inal }}-Q_{\text {initial }}$ and $l$ refers to lepton type.). The corresponding semileptonic lagrangian

$$
\mathcal{L}_{\text {oemilep }}^{W}=\frac{G}{\sqrt{2}}\left(J_{\lambda}^{+} \bar{j}_{\lambda}^{-}+J_{\lambda}^{-} j_{\lambda}^{+}+J_{\lambda}^{0} j_{\lambda}^{0}\right)
$$

where the lower-case $\dot{j}$ are the same leptonic currents as in $\mathcal{L}_{\text {lep }}^{w}$ and the upper-case $J_{\lambda}$ are phenomenological hadronic currents whose vector part $V_{\lambda}$ satisfies CVC, or conserved vector current: $\partial V_{\lambda} / \partial x_{\lambda}=0$. The weak interaction is therefore maximally parity violating, or pure left-handed $\boldsymbol{V}-\boldsymbol{A}$ for particles and pure right-handed for antiparticles.

From the structure of the leptonic currents $(V-A)$ and the assumption of an $S U(2) \otimes U(1)$ structure, we can find an appropriate representation of the generators $\mathbf{H}$. To form a 2-dimensional object with $\Delta Q=$ -1 for the $S U(2)$ left-handed weak interaction take the combined vector $\left(\begin{array}{c}\psi_{\nu_{1}} \\ \psi_{1}\end{array}\right)$; then the $S U(2)$ isospin vector of generators is $\mathbf{H}_{(2)}=\frac{1}{4} \int \phi^{\dagger}(1+$ $\left.\gamma_{5}\right) \vec{r} \psi d^{3} r$, where $\overrightarrow{7}$ are the Pauli matrices. The matrix that commutes with $\overrightarrow{\mathbf{H}}_{(2)}$ and generates electromagnetic charge is $Q_{e l}-H_{(2)}^{Z}$, where $Q_{\ell l}=$ $-\int \psi_{1}^{\dagger} \psi d^{3} r$, so

$$
H_{(1)}=\int \phi^{\dagger}\left|\frac{1}{4}\left(1-\gamma_{3}\right) r^{z}-\frac{1}{2}\right| \phi d^{3} r .
$$

Thus we see that $\boldsymbol{H}_{(1)}$ contains a right-handed piece while $\overrightarrow{\mathbf{H}}_{(2)}$ is purely 
left-handed. We can break $\psi$ ap into left-and right-handed pieces by defining $\psi_{l L}=\frac{1}{2}\left(1+\gamma_{\mathrm{b}}\right) \psi_{l}$ and $\psi_{l R}=\frac{1}{2}\left(1-\gamma_{\mathrm{b}}\right) \psi_{l}$; then

$$
\psi_{L}=\left(\begin{array}{c}
\psi_{\nu} \\
\psi_{L L}
\end{array}\right) \text { and } \phi_{R}=\psi_{I R}
$$

The spin-0 Higgs fleld should be in an $S U(2)$ doublet; 3 components will give a third helicity component to each of the $3 \mathbf{H}_{(2)}$ gauge fields and any remaining components become the artifact Higgs field. The simplest way to add the Higgs field is to define

$$
\phi=\left(\begin{array}{l}
\phi_{1} \\
\phi_{2}
\end{array}\right)\left(\phi_{1}, \phi_{2} \text { complex }\right) .
$$

The quarks also lend themselves naturally to grouping into $\Delta Q=-1$ $S U(2)$ left-handed doublets; since all quarks have masses (unlike neutrinos), they will all have right-handed $U(1)$ singlet projections also. Isospin symmetry puts $u$ and $d$ quarks in the same doublet; thereafter quark doublets are basically assigned by charge and mass (see figure 2.1.1) (Quark fields are labeled as $q \equiv \psi_{q}$, so $q_{L}=\frac{1}{2}\left(1+\gamma_{5}\right) q$, etc. .

The isospin triplet of gauge fields corresponding to $\mathbf{H}_{(2)}$ we call $\mathbf{B}_{\mu}$ and the $H_{(1)}$ singlet $C_{\mu}$. The structure constants for $S U(2)$ are $c_{i j k}=$ $\epsilon_{i j k}$ where $\epsilon_{i j k}=1$ for $i, j, k=$ any even permutation of $1,2,3 ; \epsilon_{i j k}=$ -1 for any odd permutation of $1,2,3$; and $\epsilon_{i j k}=0$ otherwise. Thus for $S U(2) c_{i j k} B_{\mu}^{j} B_{\nu}^{k}=\overrightarrow{\mathbf{B}}_{\mu} \times \overrightarrow{\mathbf{B}}_{\nu}$. (Note: The $\mathbf{B}$ transform like $\vec{f}$ and do not commute.) Then with the gauge kinetic terms $\overrightarrow{\mathrm{B}}_{\mu \nu}$ and $C_{\mu \nu}$ constructed according to 2.2.15 and using $D_{\mu}$ from 2.2.17,

$$
D_{\mu}=\partial_{\mu}-i g \vec{r} \cdot \overrightarrow{\mathbf{B}}_{\mu} Q_{(2)}-i g^{\prime} C_{\mu} Q_{(1)}
$$

where $Q_{(2)}$ and $Q_{(1)}$ are the appropriate $S U(2)$ and $U(1)$ charges of the fields acted on, we arrive at the full electroweak lagrangian

$$
\begin{aligned}
L^{E W} & =-\frac{1}{4} \overrightarrow{\mathrm{B}}_{\mu \nu}^{2}-\frac{1}{4} C_{\mu \nu}^{2}-\psi_{L}^{\dagger} \gamma_{4} \gamma_{\mu} D_{\mu} \psi_{R}-\phi_{R}^{\dagger} \gamma_{4} \gamma_{\mu} D_{\mu} \psi_{L} \\
& -q_{R}^{\dagger} \gamma_{4} \gamma_{\mu} D_{\mu} q_{R}-q_{L}^{\dagger} \gamma_{4} \gamma_{\mu} D_{\mu} q_{L}-\left(D_{\mu}^{\dagger} \phi^{\dagger}\right)\left(D_{\mu} \phi\right) \\
& -U(|\phi|)+\text { higgs-lepton and higgs-quark couplings. }
\end{aligned}
$$

$\ln L^{E W}, U(|\phi|)=\mu^{2}\left(\phi^{\dagger} \phi-\rho^{2}\right)^{2} / 4 \rho^{2}$ generates the vacuum expectation value of $\phi,\left\langle|\phi|>_{V A C}=\rho \hat{z}\right.$; and the Higgs-lepton and Higgs-quark couplings need not concern us at the moment. Terms involving $\psi$ and $q$ are taken to be summed over all lepton(quark) generations.

Since $\phi$ transforms like $S U(2) \otimes U(1)$ and has 1 arbitrary phase, we may impose $4-1=3$ constraints corresponding to a choice of gauge. If we choose these 3 constraints so that only 1 real component of $\phi(=x)$ survives (ie $\phi \rightarrow\left(\begin{array}{c}0 \\ \rho+\chi\end{array}\right)$, where $\chi$ is expanded around the VEV $=\rho$ ), this is called the Unitary gauge. In this gauge, from 2.2.24

$$
D_{\mu} \phi=\left(\begin{array}{c}
0 \\
\partial_{\mu} \chi
\end{array}\right)-i \frac{1}{2}(\rho+\chi)\left(\begin{array}{c}
g B_{\mu}^{z}-i g B_{\mu}^{y} \\
-g B_{\mu}^{x}+g^{\prime} C_{\mu}
\end{array}\right)
$$

From 2.2.28 we immediately see that the appropriate gauge feld combinstions to consider in an expansion around the $\mathrm{VEV}=\rho$ are (suggestively defined)

$$
\begin{aligned}
W_{\mu} & =\frac{1}{\sqrt{2}}\left(B_{\mu}^{s}-i B_{\mu}^{y}\right) \quad \text { and } \\
Z^{\mu} & =\cos \theta_{W} B_{\mu}^{z}-\sin \theta_{W} C_{\mu}
\end{aligned}
$$

where

$$
\tan \theta_{w} \equiv \frac{g^{\prime}}{g} .
$$

$\tan \theta_{W}$ is the ratio of the $U(1)$ to the $S U(2)$ coupling constant, and the lower component of 2.2 .28 may be written as $-g Z_{\mu} / \cos \theta_{w}$. We define 
the photon field orthogonal to $Z_{\mu}$ in 2.2 .27

$$
A_{\mu}=\sin \theta_{W} B_{\mu}^{s}+\cos \theta_{W} C_{\mu} \text {. }
$$

$W^{+}$is the conjugate field to $W^{-}$, so

$$
W^{+}=\frac{1}{\sqrt{2}}\left(B_{\mu}^{x}+i B_{\mu}^{y}\right) .
$$

$\left(D_{\mu}^{\dagger} \phi\right)\left(D_{\mu} \phi\right)$, reexpressed in terms of $W^{ \pm}$and $Z$, gives terms

$$
\begin{gathered}
-\frac{1}{2} \rho^{2} g^{2} W_{\mu}^{+} W_{\mu}-\frac{1}{2} \rho^{2} g^{2} Z_{\mu} Z_{\mu} / 2 \cos ^{2} \theta_{W} \text { or } \\
-M_{W}^{2} W_{\mu}^{+} W_{\mu}^{-}-\frac{1}{2} M_{Z}^{2} Z_{\mu} Z_{\mu},
\end{gathered}
$$

from which we see that the $W^{ \pm}$boson has mass $M_{W}=\rho g / \sqrt{2}$ and the $Z$ boson has mass

$$
M_{Z}=\frac{M_{W}}{\cos \theta_{W}} .
$$

The coupling of the gauge fields to the quarks and leptons via the covariant derirative 2.2.24 (taking for example a lepton doublet) leads to:

$$
\begin{aligned}
-\psi_{L}^{\dagger} \gamma_{4} \gamma_{\mu} D_{\mu} \psi_{L} & =-\psi_{L}^{\dagger} \gamma_{4} \gamma_{\mu} \partial_{\mu} \psi_{L} \\
& +\frac{i}{2} \psi_{L}^{\dagger} \gamma_{4} \gamma^{\mu}\left(\begin{array}{c}
g B_{\mu}^{x}-g^{\prime} C_{\mu} g B_{\mu}^{x}-i g B_{\mu}^{y} \\
g B_{\mu}^{x}+i g B_{\mu}^{y}-g B_{\mu}^{x}-g^{\prime} C_{\mu}
\end{array}\right) \psi^{L},
\end{aligned}
$$

a kinetic term and the gauge field coupling. Using the definitions for $W_{\mu}^{ \pm}$, $Z_{\mu}$, and $A_{\mu}$ (2.2.27 and following) the gauge coupling term is reexpressed 28

$$
\frac{i}{\sqrt{2}} \phi_{L}^{\dagger} \gamma_{4} \gamma_{\mu} g\left(\begin{array}{cc}
\frac{z_{\mu}}{\sqrt{2} \cos \theta_{W}} & W_{\mu}^{-} \\
W_{\mu}^{+} & \frac{1}{\sqrt{2}}\left(-2 \sin \theta_{W} A_{\mu}-\cos \theta_{W}\left(1-\tan ^{2} \theta_{W}\right) Z_{\mu}\right.
\end{array}\right) \phi_{L}
$$

Reading off the top $(1,2)$ component we see that the $\boldsymbol{W}^{-}$field couples as

$$
\begin{gathered}
\frac{i}{\sqrt{2}} \psi_{\nu_{l}}^{\dagger} \gamma_{4} g \gamma_{\mu} W_{\mu}^{-} \frac{1}{2}\left(1+\gamma_{5}\right) \psi_{l} \\
=\frac{g}{2 \sqrt{2}}\left(W_{\mu}\right)\left(j_{\mu}^{+}\right)
\end{gathered}
$$

where $j_{\mu}^{+}$is equivalent to the phenomenological lepton current given in 2.2.19. Similarly reading off the photon coupling from the $(2,2)$ component of the matrix in 2.2 .34 , we have

$$
\begin{gathered}
-\frac{i}{2}(2) \frac{1}{2}\left(1+\gamma_{5}\right) \psi_{l}^{\dagger} \gamma_{4} \gamma_{\mu} g \sin \theta_{W} A_{\mu} \frac{1}{2}\left(1+\gamma_{5}\right) \psi_{l} \\
=\frac{1}{2}\left(-i g \sin \theta_{W} A_{\mu} \psi_{l}^{\dagger} \gamma_{4} \gamma_{\mu} \psi_{l}\right) .
\end{gathered}
$$

With the addition of another term identical to 2.2 .38 from the $-\psi_{R}^{\dagger} \gamma_{4} \gamma_{\mu} D_{\mu} \phi_{R}$ term in $L^{E W}$, this is

$$
\mathcal{L}_{I A}=-i g \sin \theta_{W} A_{\mu} j_{\mu}^{E M}
$$

where $j_{\mu}^{E M}$ is the electromagnetic current $\phi_{l}^{\dagger} \gamma_{4} \gamma_{\mu} \phi_{l}$. From 2.2.39 we see that

$$
e=g \sin \theta_{w}
$$

where $e$ is the electric charge

From the symmetry of the electroweak lagrangian 2.2 .25 between quarks and leptons, with quarks grouped in $S U(2)$ doublets as in figure 2.1.1, we see that the hadron currents coupling to $W_{\mu}^{ \pm}$must have the same form as the leptonic current in $\mathbf{2 . 2 . 3 5}$, or that

$$
J_{\mu}^{+}=i q_{u}^{\dagger} \gamma_{4} \gamma_{\mu}\left(1+\gamma_{5}\right) q_{d^{\prime}}+i q_{0}^{\dagger} \gamma_{4} \gamma_{\mu}\left(1+\gamma_{\delta}\right) q_{0^{\prime}}
$$




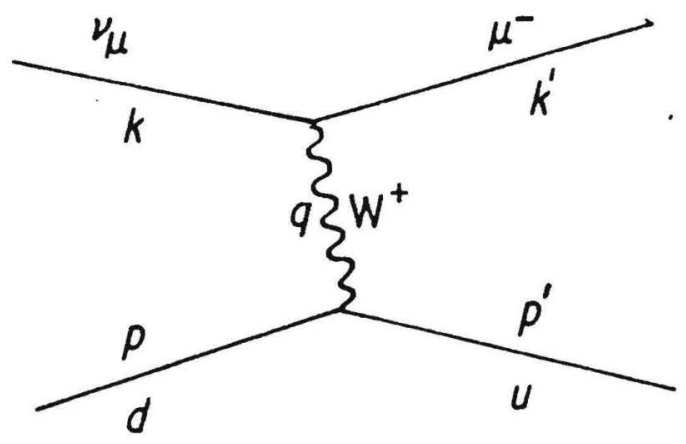

FIGURE 2.2.1.

An example of single $\boldsymbol{W}$-exchange with $J_{\mu}^{-}$defined as the conjugate current. The neutral currents, both leptonic and hadronic, are more complicated due to the mixing of the photon and the $Z$.

The second order weak $\Delta Q= \pm 1$ interaction will be from single $W$-exchange, as shown in the example in figure 2.2.1. Diagrams such as figure 2.2.1 will come from the contraction of the $W_{\mu}^{-}$and $W_{\mu}^{+}$currents (evaluated at different space-time points) between the interaction term 2.2.36 and an equivalent term with the hadron current $J_{\mu}^{+}$given in 2.2.41:

$$
M \sim \frac{g^{2}}{8} J_{\lambda}^{+}(y) W_{\lambda}(y) W_{\mu}^{+}(x) J_{\mu}^{-}(x) .
$$

The $W$ propogator in 2.2 .42 is the propogator for a massive spin-1 particle

$$
-i \frac{\delta_{\lambda \mu}+q_{\lambda} q_{\mu} / M_{W}^{2}}{q^{2}+M_{W}^{2}-i \epsilon} .
$$

In the low energy limit (applicable to E546), $q^{2}<M_{W}^{2}$, this propogator reduces to $-i \delta_{\lambda_{\mu}} / M_{W}^{2}$ and the matrix element reduces to

$$
M \sim \frac{\rho^{2}}{8 M_{W}^{2}} J_{\mu}^{+} j_{\mu}^{-},
$$

equivalent to the phenomenological semileptonic lagrangian 2.2.20. The phenomenological lagrangisn 2.2.20, with the charged hadron currents given by 2.2 .41 and its conjugate, is adequate for all weak-interaction phenomena studied in this dissertation.

\subsection{The strong Interaction.}

The strong interaction is responsible for binding nucleons into nuclei, and like the weak interaction, this nuclear binding force is a short range 
force. Only quarks couple to the strong interaction. The strong interaction is also assumed to bind quarks into hadrons. The fact that no free quark has ever been observed suggests that quarks are permanently "confined" in hadrons, and this confinement considersbly complicstes the problem of understanding the strong force. As a result the strong force is much less well understood than the electroweak force.

A candidate theory for the strong force does exist, however. The dramatic successes of the electroweak theory and the proven renormalizability of $S U(N)$ gauge theories suggests an $S U(N)$ gauge theory as a candidate $^{|28|}$. The rank $N$ of the candidate theory can be determined from $e^{+} e^{-}$scattering: the ratio $R=\sigma\left(e^{+} e^{-} \rightarrow q \bar{q} \rightarrow\right.$ hadrons $) / \sigma\left(e^{+} e^{-} \rightarrow\right.$ $\mu^{+} \mu^{-}$) is equal to the sum of the quark charges squared (of all quarks light enough to be produced for given s), multiplied by a constant scale factor $C_{N}$,

$$
R=C_{N} \sum_{q-u, 0, d, \ldots} Q_{q}^{2} .
$$

Because we are just counting the number of quarks that can couple to the virtual gamma $\left(\gamma^{\circ}\right)$ in figure 2.3.1, $C_{N}$ counts the number of "types" of up quark, down quark, and so on. Experimentally $C_{N}=3^{[20]}$, implying that the rank of the symmetry group (equivalent to the number of types of "strong" charge) is $N=3$.

The 3 charges suggest the 3 primary colors, and the $S U(3)$-invariant gauge theory of the strong interactions is called Quantum ChromoDynamics, or QCD. An $S U(3)$-invariant lagrangian is built in exactly the same way as in the preceding section, and the result is

$$
L^{S}=-\frac{1}{4} F_{\mu \nu}^{i} F^{i \mu \nu}-\psi^{\dagger} \gamma_{4}\left(\gamma_{\mu} D_{\mu}+m\right) \psi_{;}
$$

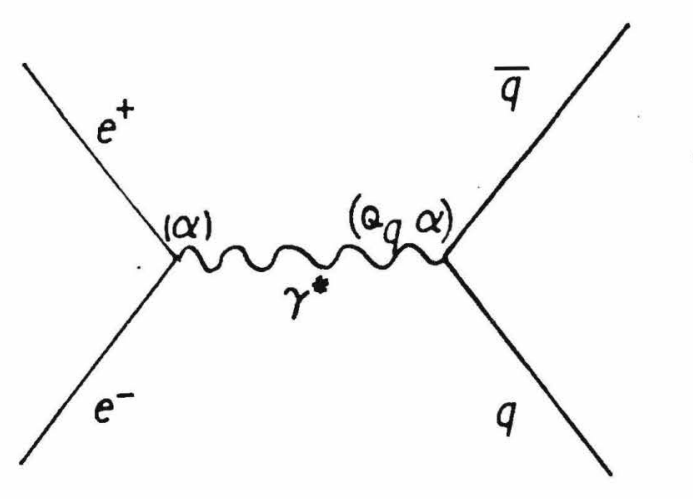

FIGURE 8.8.1.

$e^{+} e^{-} \rightarrow$ hadrons via a virtual photon. $Q_{q}$ is the electric charge of quark $q$ in units of $e$. 
with

$$
\begin{gathered}
\phi=\left(\begin{array}{l}
\phi_{1} \\
\phi_{2} \\
\phi_{3}
\end{array}\right), \quad\left(\phi_{1}, \phi_{2}, \phi_{3}=\text { Dirac spinors }\right) ; \\
F_{\mu \nu}^{i}=\partial_{\mu} A_{\nu}^{i}-\partial_{\nu} A_{\mu}^{i}+g c_{i j k} A_{\mu}^{j} A_{\nu 1}^{k}
\end{gathered}
$$

where the $A_{\mu}^{j}$ are the gauge fields (there are $3^{2}-1=8$ generators and so 8 gauge field in $S U(3)$, called gluons), the $c_{i j k}$ are the $S U(3)$ structure constants; and

$$
D_{\mu}=\partial_{\mu}-i g H^{i} A_{\mu}^{i},
$$

with the $H^{i}=\frac{1}{2} \lambda^{i}$ the 8 generators of $S U(3)$. Unlike the weak interaction, $S U(3)$ is considered to be an unbroken symmetry, so the 8 gauge flelds remain massless.

The origin of conflnement is uncertain, but $S U(3)$ does possess suggestive properties ${ }^{[30]}$. A perturbation expansion of QCD to order $g_{0}^{2}$, where $g_{0}$ is the unrenormalized QCD coupling constant, shows that the strong coupling constant scales with length as $[31]$

$$
\frac{g_{l}^{2}}{\theta_{L}^{2}}=\frac{1}{1+\frac{g^{2}}{8 \pi^{2}}\left(11-\frac{2}{3} n\right) \ln \psi^{\prime}},
$$

where $L$ and $l$ are two length scales and $n$ is the number of quark flavors (the $-\frac{2}{3} n$ term comes from virtual quark pair charge screening, just as $e^{+} e^{-}$pairs screen the bare charge in QED). From 2.3.4 we see that

$$
\lim _{1 / L \rightarrow 0} \frac{g_{i}^{2}}{g_{L}^{2}}=0
$$

which shows that at very short distance scales (equivalent to high momentum transfers) quarks act like free particles, a property of $S U(3)$ called asymptotic freedom. Experimental results bear out this property up to currently accessible momenta. Alternatively, as $l / L$ increases, so does $g_{l}^{2} / \rho_{L}^{2}$, implying a stronger coupling and ultimately, confinement. This increased coupling indicates that the QCD vacuum is antiscreening, provided $n<17$.

Suppose that the $Q C D$ vacuum is perfectly polarizable. For screening, this would correspond to $\kappa \rightarrow \infty$, where $\kappa$ is the dielectric constant. But for antiscreening, $\alpha \rightarrow 0$. The total energy in the field $\sim \int \overrightarrow{\mathbf{E}} \cdot \overrightarrow{\mathbf{D}} d^{3} r$, and $\overrightarrow{\mathbf{E}}$ (the color electric fleld) in turn $\sim \frac{1}{\kappa}$, so as $x \rightarrow 0$ the energy of an isolated color charge $\rightarrow \infty$. This in turn implies that isolated color charges are forbidden. But for a color multipole with net color charge 0 , we can form a small "hole" in the $\kappa=0$ medium. In the hole $\kappa=1$; outside $\kappa=0$. If we arrange the color electric field so that is parallel to the surface at the surface (see figure 2.3.2), then $\overrightarrow{\mathbf{D}}=\mathbf{0}$ outside and the charge distribution has a finite energy. This simple argument implies that quarks only come in color charge 0 combinations, called color singlets. This can occur by combining a quark and an antiquark $(q \bar{q})$ or three quarks antisymmetric in their color indices $(q q q)$. Similarly 6-quark combinations are allowed, along with higher color multipoles.

$u, d$, and $s$ are the lowest-lying quarks and the only ones that can be produced with any frequency at WA51 and E546 experimental energies. Arranging these 3 flavors in $q \bar{q}$ and $q q q$ combinations produces the $S U(3)$ flavor group $S U(3)_{F} . S U(3)_{F}$ has a primary 3 representation and a conjugate $\overline{3}$ representation (which are not equivalent). The $q \bar{q}$ representations form $3 \otimes \overline{\mathbf{3}}=\mathbf{8}_{M} \oplus 1_{A}$, an octet and a singlet in quark flavors; the qqq representation forms $3 \otimes 3 \otimes 3=10_{S} \oplus 8_{M} \oplus 8_{M} \oplus 1_{A}$, or decuplets, 
octets, and singlets. The overall symmetry group with the addition of $S U(2)$ spin is $S U(6)$. Hadrons do indeed arrange themselves in these combinations.

Mesons arise from $q \bar{q}$ combinations, baryons from $q q q$ combinations. The lowest-lying meson octets $\left(J^{P}=0^{-}\right.$and $\left.J^{P}=1^{-}\right)$and singlets are shown in figure 2.3.3 in standard $S U(3)$ representation. The lowest-lying baryon decuplet and octet are shown in figure 2.3.4. Because the singlets have identical quant um numbers to the central states in the octet, all these states are mixed states. (Note: if charm quarks are included, $S U(4)_{F}$ must be used, producing $4 \otimes \overline{4}=15_{M} \oplus 1_{A}$. Because $c$ quarks have $m_{c} \sim 2$ $\mathrm{GeV} / \mathrm{c}^{2}$, they are greatly suppressed at these energies and ignored here).

The process by which quarks produced in an interaction combine themselves into hadrons is only poorly understood. The idea of confined color flux discussed above suggests a simple model. After a quark interacts, it moves away from other quarks involved in the interaction. Color flux binding the quark to the other quarks is squeezed down to a color flux tube by the vacuum dielectric. The energy density in this flux tube may create $q \bar{q}$ pairs, which combine to form mesons, etc. As a second order effect, diquark-antidiquark pairs may be created and form baryons (a diquark is a $q q$ or $\not \bar{q}$ pair, and is in the $\mathbf{3}$ or $\mathbf{3}$ representation, respectively). Thus a string of mesons and possibly baryons is created with their average momentum along the original quark direction, called a jet. Such an effectively 1-dimensional picture of hadronization is a "string model." Lattice gauge theory calculations indicate a string model is a good $S U(3)$ color model approximation providing dimensions less than the transverse

dimension of the flux tube are not considered ${ }^{|32|}$. How $q \bar{q}$ pairs should be

\section{FIGURE 2.8.8.}

Quark confinement from a perfect vacuum color dielectric. 
41
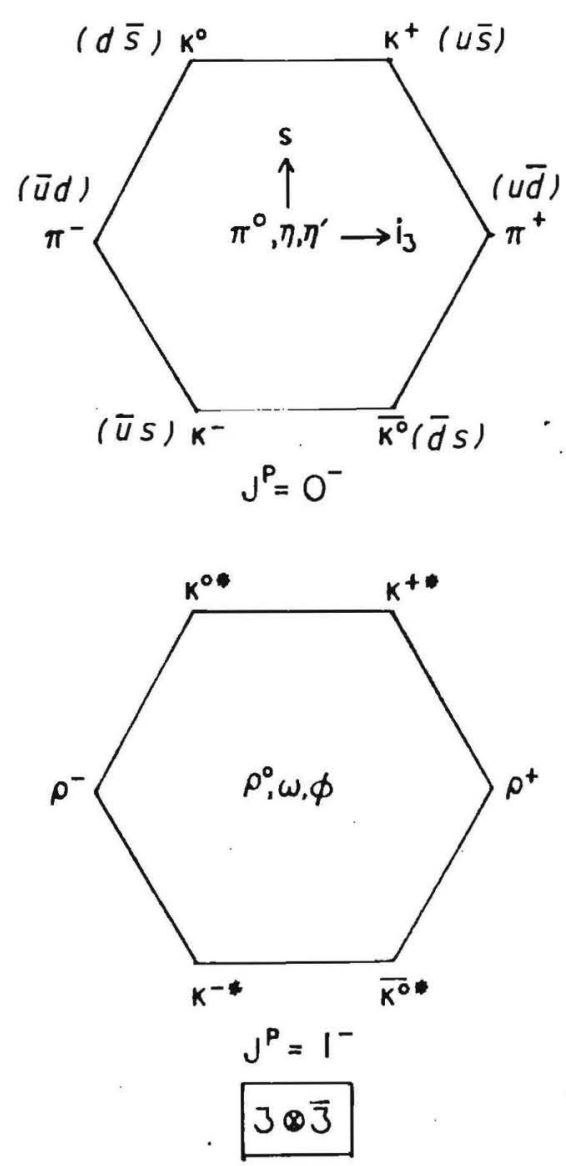

FIGURE 8.8.8.

The $J^{P}=0^{-}$and $J^{P}=1^{-}$meson $(q \bar{q})$ octets and corresponding singlets.
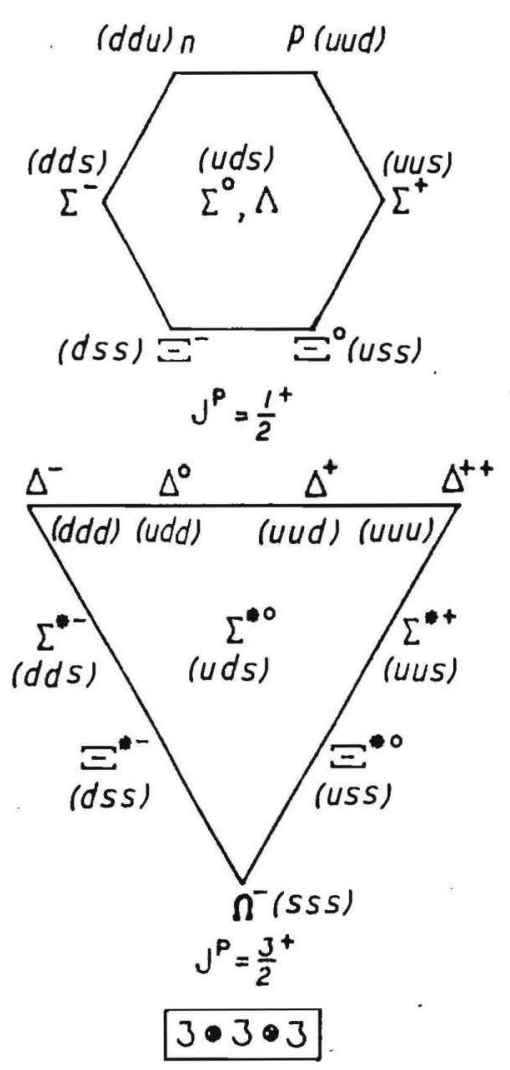

FIGURE 2.8.4.

The $J^{P}=\frac{1}{2}^{+}$baryon (qqq) octet and singlet, and the $J^{P}=\frac{8}{2}^{+}$baryon decuplet. 
created and how the arailable kinetic energy should be distributed among them is unknown; simply distributing them according to the phase space available evidently works as well as any method.

Because quarks are conflned objects, one must be careful in dealing with their masses, as the mass obtained depends intimately on the confinement mechanism, which is not well understood. In addition, quark masses are not eigenstates of the weak hamiltonian, and the quarks appearing in the weak $S U(2)$ doublets are mixtures of the strong interaction quarks. If we consider only $u, d, s$, and $c$ quarks, then a unitary mixing between quark doublets will fall in $U(2)$, which has 4 real parameters. There are 3 arbitrary relative phases between $u, d, s$, and $c$, leaving a 1-parameter transformation. This unitary matrix is ${ }^{[33]}$

$$
\left(\begin{array}{l}
d^{d} \\
d^{\prime}
\end{array}\right)=\left(\begin{array}{cc}
\cos \theta_{c} & \sin \theta_{c} \\
-\sin \theta_{c} & \cos \theta_{c}
\end{array}\right)\left(\begin{array}{l}
d \\
d
\end{array}\right)
$$

where the mixing angle $\theta_{c}$ is the "Cabbibo angle." (If the bottom quark is added, then a more complicated model using $U(3)[9-5=4$ parameters $]$ is needed ${ }^{[34]}$ )

The short range character of the nuclear binding force is viewed as a secondary effect of QCD; $q q q$ color-singlet baryons with "hidden" color create a secondary fleld of $q \bar{q}$ pairs around them (of which the lowest-lying state is the pion), and exchange of these massive virtual pions produces the short-range nuclear binding force.

f 2.4. High Energy Neutrino Interactions.

${ }^{[35]}$ The reaction

$$
\nu+\alpha \rightarrow l+\beta,
$$

where $\alpha$ and $\beta$ are hadronic systems, and the momenta are labeled in figure 2.4.1, is referred to as a Charged Current, or CC neutrino interaction. For the energies available in E548, it is described by the semileptonic lagrangian given in section 2.2. The square of the matrix element (summed over the helicities of $\alpha, l$, and $\beta$ ) will be

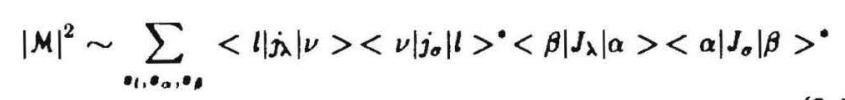

which we may group into a lepton tensor for the lepton currents $j$ and a hadron tensor for the hadronic currents $J$ :

$$
|M|^{2} \sim \sum L_{\alpha \beta} H_{\alpha \beta}
$$

In the limit where we can ignore the lepton mass $m_{l}$, the leptonic current is conserved and $\partial_{\lambda} \dot{j}_{\lambda}=0$, or equiralently $q_{\lambda} \dot{j}_{\lambda}=0$. This implies that any component of $H_{\alpha \beta}$ involving $q_{\lambda}$ drops out of $|M|^{2}$, leaving only 3 tensor combinations of the hadronic system that can contribute to $H_{\alpha \beta}$ :

$$
\delta_{\alpha \beta}, \quad p_{\alpha} p_{\beta}, \quad \text { and } \epsilon_{\alpha \beta \mu \nu} p_{\mu} p_{\nu}^{\prime}
$$

The cross section for reaction 2.4.1 will therefore contain 3 scalar functions playing the role of "form factors" which, because of the local nature of the interaction, can be functions of $q^{2}$ and one other Lorentz invariant at the hadronic vertex. Two Lorentz invariants that are commonly used in neutrino reactions are

$$
x_{B J}=-\frac{q^{2}}{2 p \cdot q}=\left(\frac{-q^{2}}{2 M \nu}\right)_{L A B}
$$


and

$$
y=\frac{q \cdot p}{k \cdot p}=\left(\frac{E_{\nu}-E_{l}}{E_{\nu}}\right)_{L A B}
$$

$\left(x_{B} \mathrm{~J}\right.$ is hereafter referred to as $\left.x\right)$. In terms of these invariants, and adding the appropriate phase space factor, the cross section can be written

$$
\begin{aligned}
\frac{d^{2} \sigma}{d x d y}=\frac{M E_{\nu}}{\pi} G^{2}\left[x y^{2} F_{1}\left(q^{2}, x\right)+\left(1-y-\frac{M}{2 \nu} x y^{2}\right) F_{2}\left(q^{2}, x\right)\right. \\
\\
\left. \pm x y\left(1-\frac{1}{2} y\right) F_{3}\left(q^{2}, x\right)\right],
\end{aligned}
$$

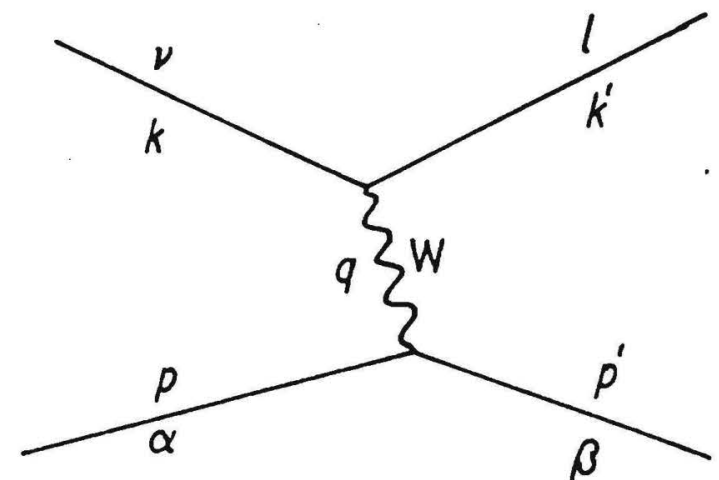

FIGURE \$.4.1.

High energy neutrino charged current (CC) diagram. with $M$ the nucleon mass, $\nu=q_{0}, G$ the fermi constant, the upper sign of \pm for $\nu$ and the lower sign for $\nu$.

If we assume that we are at a large enough $q^{2}$ that all mass scales drop out of the problem, then $M / \nu \rightarrow 0$ and the $F_{i}$ become functions of dimensionless variables only, i.e. only $x$. This gives the scaling limit

$\frac{d^{2} \sigma}{d x d y}=\frac{M E_{\nu}}{\pi} G^{2}\left[x y^{2} F_{1}(x)+\left(1-y^{2}\right) F_{2}(x) \pm x y\left(1-\frac{1}{2} y\right) F_{3}(x)\right]$. (2.4.8)

There is a natural interpretation of $x$ in terms of the nucleus' constituent quarks, gluons, and $q \bar{q}$ ocean. The asymptotic freedom property of the strong interaction lets us treat the quark as a free particle during the interaction and worry about hadronization later. Consider reaction 2.4.1 when $\alpha$ is a quark and $\beta$ another quark, and the 4-momenta are $k+k^{\prime} \rightarrow p_{q}+p_{q}^{\prime}$, with $k-k^{\prime}=q=p_{q}^{\prime}-p_{q}$ the exchanged momentum. Then from

$$
p_{q}^{\prime}=\left(q+p_{q}\right)^{2}=p_{q}^{2}+q^{2}+2 p_{q} \cdot q
$$

we have

$$
q^{2}=-2 p \cdot q
$$


where since we are at a momentum beyond all mass scales, $p_{q}^{\prime 2}=p_{q}^{2}$ From the original definition of $x$ (2.4.5), we see that

$$
x=\frac{q \cdot p_{q}}{q \cdot p} \text { or } \quad x(q \cdot p)=q \cdot p_{q},
$$

where $p$ is the momentum of the nucleon. Since all transverse momenta are limited by the only transverse scale available, the confinement radius, we have $p_{q} r \sim \mathcal{O}\left(1 / R_{N}\right)$, where $R_{N}$ is the radius of the nucleon. If $\overrightarrow{\mathrm{p}}$ is along the $\hat{z}$ axis, then we have

$$
\begin{aligned}
& p_{0}=p_{z}+O\left(\frac{M^{2}}{p_{x}}\right) \text { and } \\
& p_{q 0}=p_{q z}+O\left(\frac{m_{q}^{2}}{p_{q x}}, \frac{p_{q} T}{p_{q z}}\right)
\end{aligned}
$$

so that $p_{q}$ is parallel to $p$ in the frame $\overrightarrow{\mathbf{p}} \rightarrow \infty$. Thus in this frame relation 2.4.11 becomes

$$
p_{q}=x p
$$

i.e. $x$ is the fraction of the nucleon momentum carried by the quark. Because as $x \rightarrow 1$ for a quark, that quark must carry the full helicity of the nucleon, all ocean quarks must be concentrated in the $x \ll 1$ region (from their definitions 2.4.5 and 2.4.6, we see that $x$ and $y$ can range between 0 and 1). Finally, we can rewrite the structure functions $F_{i}(x)$ in terms of combinations of quark structure functions $q_{m}(x)$, where $m=$ $u, d, s, c, \ldots$, and write down reaction 2.4.1 purely in terms of fundamental leptons and quarks. $\left(q_{m}(x) d x\right.$ is the probabilty of finding an $m$ quark with momentum fraction between $x$ and $x+d x$ ). In the scaling limit, we find that

$$
F_{2}(x)=2 x F_{1}(x)
$$

a)

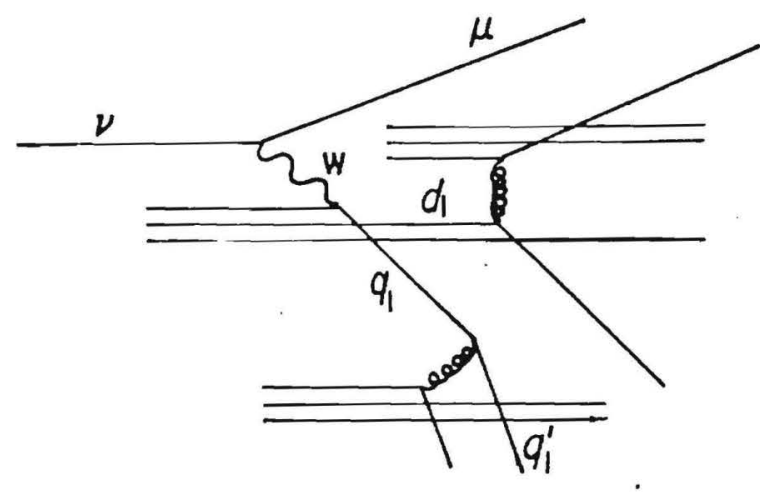

b)

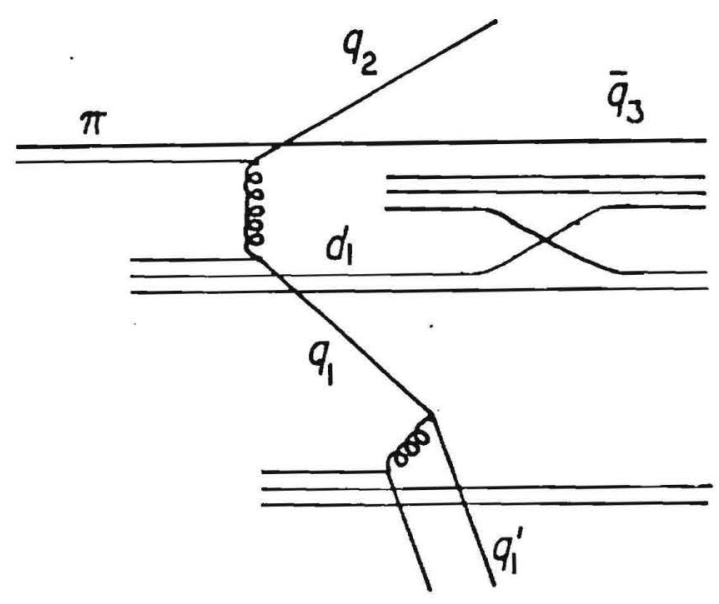

FIGURE 2.5.1.

Schematic nuclear reinteraction diagrams. $(a)^{(-)} \mathrm{Ne}$. (b) $\pi^{ \pm} \mathrm{Ne}$. 
called the Callan-Gross relation ${ }^{|86|}$.

\section{2.5. Nuclear Effects.}

If the target in a high energy interaction is a nucleus instead of a nucleon, then the system produced in the interation has the potential of reinteracting with the remaining nucleons in the nucleus. Nuclear reinteractions therefore probe the properties of an hadronic system shortly after its creation, ie over distance scales of a few fermis. This can be a valuable tool for analy zing the hadronization mechanism of quarks, a process which is currently poorly understood. Figure 2.5 .1 gives a schematic impression of how such reinteractions can occur in $\left(\bar{\nu}^{\prime} \mathrm{Ne}\right.$ and $\pi^{ \pm} \mathrm{Ne}$.

If we assume that the momenta involved in these interactions is large enough that we can ignore transverse momentum scales relative to longitudinal momentum scales, then a high-momentum quark will leave behind a string of particles formed from $q \bar{q}$ pair production in the color flux tube. This string of particles with average momentum along the initial quark direction is called a "jet". In neutrino scattering (figure 2.5.1(a)), if there are no rescatters then there are two jets, from the scattered quark $\left(q_{1}\right)$ and the remnant diquark $\left(d_{1}\right)$. In pion scattering (figure 2.5.1(b)) if there are no rescatters then there are four hadronic jets, one each from the two interacting quarks $\left(q_{1}\right.$ and $\left.q_{2}\right)$, one from the remnant pion quark ( $\bar{q}_{3}$ in the figure), and one from the remnant diquark $\left(d_{1}\right)$. (In both cases the possibility of hard gluon emmision has been ignored. Also it should be noted that the momenta involved in our pion scattering experiment are too small to resolve four jets.) Nuclear reinteractions can, in turn, add arbitrary complications to the final state, as suggested in the figure.

The average number of interactions in the nucleus (in this section called $(\nu))$ is shown in appendix $\mathrm{A}$ to be

$$
\langle\nu\rangle=\frac{A \sigma_{N}}{\sigma_{A}},
$$

where $A$ is the number of nucleons in the nucleus, $\sigma$ is the cross section for a given reaction, and $\sigma_{N}$ and $\sigma_{A}$ refer to the nucleon and the nucleus, respectively. Using this definition, $\langle\nu\rangle$ for $\pi^{ \pm} \mathrm{Ne}$ is about 1.5 , showing that nuclear reinteractions are indeed a significant factor in particle production off auclei. However, nuclei multiply particle production much more slowly than would be expected in a simple cascade model. In such a model each interaction is exactly equivalent to a hadron-nucleon interaction, and all produced secondaries are assumed to have cross sections equivalent to that type of secondary incident on a nucleon in a laboratory scattering experiment. Experimentally we find that $\sigma(A) \sim A^{2 / 8} \sigma_{N}$, where $\sigma(A) \sim$ $A \sigma_{N}$ in a simple cascade model.

This is a plausible result in terms of quark diagrams, such as those in figure 2.5.1. The hadronization process discussed in section 2.3 will occur over significant length scales. If a scattered quark is unlikely to interact until "dressed" as a hadron, then we expect little rescattering if the quark has sufficient energy to leave the nucleus before hadronizing. Viewed in another manner, in the string model we stretch a tube of color flux between the quark and the diquark remnant of the nucleon the quark was scattered out of. If the quark is a low energy scatter, the flux tube will be mainly contained in the nucleus and $q \bar{q}$ pairs formed in the tube can reinteract. A high energy quark will have most of the flux 
tube outside of the nucleus and little reinteraction will occur. The lack of a simple cascading picture indicates that few $q \bar{q}$ pairs are formed in the reinteraction region.

These suppression arguments involve a fundamental scale larger than the nuclear radius. Typically they can be thought of as formation-time arguments, where some time lag $\Delta t$ must elapse before a reinteraction can occur. For high energies this time lag is Lorentz dilated to give a characteristic time

$$
\tau \sim \frac{E}{m} \Delta t
$$

which scales like the energy involved.

There are several fundamental differences between $(\bar{\nu}) \mathrm{Ne}$ and $\pi^{ \pm} \mathrm{Ne}$ scattering relevant to reinteraction effects. Because the $(\bar{\nu}) \mathrm{N}$ cross section is so small (about $10^{-0} \mathrm{mb}$ at the energies of this experiment ${ }^{|37|}$ ), $\mathrm{a}^{(} \bar{\nu}^{\prime} \mathrm{Ne}$ interaction is equally likely to occur anywhere in the Ne nucleus, and so on the average the produced system will traverse $r_{N e}$, the radius of a Ne nucleus, before exiting the nucleus. A pion, on the other hand, has a significant probability of interacting before the halfway point. Given that the $\pi^{ \pm} \mathrm{N}$ cross section is $\approx 21 \mathrm{mb}$ (inelastic) for our experimental energies ${ }^{|88|}$, and treating the Ne nucleus as a dense ensemble of nucleon matter with $r_{0}=(1.12 \mathrm{fm}) A^{1 / 3}$, we calculate that on the average the $\pi^{ \pm}$-incident produced system must traverse roughly $1 \frac{1}{3}$ to $1 \frac{1}{2}$ more path length than the neutrino-incident produced system. Assuming that the probability of reinteracting $\sim$ the pathlength traversed, we expect $1 \frac{1}{3}$ to $1 \frac{1}{2}$ more reinteractions in $\pi^{ \pm} \mathrm{Ne}$ than in $\left(\bar{\nu}^{-}\right) \mathrm{Ne}$ when compared at identical hadronic CM energies.
Another fundamental difference of $\left(\bar{\nu}^{-}\right) \mathrm{Ne}$ and $\pi^{ \pm} \mathrm{Ne}$ is the remnant quark of the pion (labeled $\bar{q}_{3}$ in figure 2.5.1), which has no analog in ' $(\bar{\nu}) \mathrm{Ne}$. If this quark carries a significant fraction of the pion's momentum, it can create a forward jet (relative to the incident beam) where no such jet exists in the ${ }^{(-)^{\prime}} \mathrm{Ne}$ system. If we assume that partons must be close together in momentum space in order to interact, the remnant pion quark will frequently carry a large fraction of the pion momentum. We thus expect to often see strongly forward particles in $\pi^{ \pm} \mathrm{Ne}$ that have only minimal connection with the interaction (Evidence of this "remnant quark" effect is seen in the $\pi-\nu$ comparison and discussed in section 7.3).

Finally, except for the flavor coupling of the $W$ current, the remnant diquark ( $d_{1}$ in figure 2.5.1 for both systems) should have the same properties for both systems. Thus both systems are expected to bear strong similarities in the backward hadronic CM hemisphere. Because reinteraction products in both systems result from quarks with similar properties reacting with nuclear constituents, we expect nuclear effects will be concentrated almost exclusively in the backwards hadronic CM hemisphere, and the resulting reinteraction system will be similar for $\pi^{ \pm} \mathrm{Ne}$ and $(\bar{\nu}) \mathrm{Ne}$. One should note that reinteractions produce further remnant diquarks that will generate more baryons in the final state, some of which will be protons with significant momenta. These protons, which cannot be identifled by ionisation alone, are identifled and studied in this experiment via an isospin subtraction technique.

There exists no good description of nuclear reinteraction effects in terms of fundamental quarks and gluons, due to the close tie between reinteraction effects and the unsolved confinement problem. Most models 
of reinteraction effects are phenomenological and describe the problem in terms of bulk properties of the nucleus or in terms of some statistical average. This can be a useful approach, permitting us to average over very complicated initial states and final state evolution equations, to perhaps gain some insight into the statistical properties of the ensemble as a whole. To give some flavor of these phenomenological models, I will briefly describe several such models here.

\section{A. The Hydrodynamical Model.}

This model considers longer time scales, where $1 / \tau>$ the mean free path in nuclear matter, and is a classical model. The nucleus is treated as an ideal relativistic fluid. When the collision occurs, the nuclear fluid is first compressed and then expands; this corresponds to a collision with a target of mass $\langle\nu\rangle \cdot M_{\text {nucleon }}$. This model is originally due to Landau, and will be called the Landau Hydrodynamical Model (LHD) ${ }^{|30|}$.

In the model the incident particle is taken to have some effective cross section $\pi a^{2}$. The amount of nuclear fluid involved in the interaction is then that in a tube $\pi a^{2} d$, where $d$ is the Lorentz contracted length of the tube, $d=\left(m / E_{c m}\right) d_{0}$ where $m$ is the mass of the incident particle, $E_{c m}$ is the hadronic CM energy, and $d_{0}$ is the rest frame length of the tube. The picture is of 1 "blob" of nuclear matter fluid interacting with $\nu$ "blobs" of nuclear matter in the nucleus, for a total of $\nu+1$ "blobs"
Viewed from the equal velocity frame of the projectile and target, the sequence of events in a collision is as follows:

(1) The target is impacted and both projectile and target start to compress and heat (the compressed fluid is stationary). The compression occurs behind a shock wave that spreads out from the contact point at a velocity $v_{\mathrm{s}}=c_{0}^{2}$, where $c_{0}$ is the speed of sound in nuclear matter in units where $c=1$.

(2) Particle emission begins when the rear-going shock wave encounters the end of the projectile fluid and when the temperature of the fluid $\approx T_{c}$, the critical temperature $\left(T_{c} \approx m_{m}\right.$, the characteristic energy of free hadrons). A rarefaction wave starts forward with velocity $c_{0}\left(>v_{s}\right)$.

(3) If the tube has less that $\nu_{e}$ "blobs" in it, the forward shock wave reaches the end of the tube before being overtaken by the rarefaction wave, and particle production proceeds inward from both sides of the tube. In this case the tube will be isothermic before reaching $T_{e}$ and the ratio of particle formation to the hadron-nucleon rate will depend only on the relative volume of nuclear fluid in hadron-nucleus compared to hadron-nucleon:

$$
R\left(\nu<\nu_{c}\right)=\frac{1}{2}(\nu+1) .
$$

(4) If $\nu>\nu_{e}$, then the rarefaction wave overtakes the forward shock wave and the isothermal case no longer applies. One must solve the full hydrodynamic equations with appropriate boundary conditions, resulting in a complicated $R$ of the form

$$
R\left(\nu>\nu_{c}\right) \sim a+b\left(\nu-\nu_{0}\right)^{a},
$$

where $a, b$, and $a$ are functions of $c_{0}$, and $\nu_{0}$ is a function of $\nu_{c}$ and $c_{0}$. 
The critical value of $\nu, \nu_{c}$, is

$$
\nu_{c}=\frac{1+c_{0}}{1-c_{0}}
$$

\section{B. The Diffractive Excitation Model.}

The diffractive excitation model (DEM) ${ }^{[40]}$ considers the special case of a cascade model where the secondary interactions are of a diffractive nature. In a simple cascade model, all the interaction products have the same probability of interacting at any order of the cascade (ie the cross section $\sigma$ is a constant for all interactions). This leads to a linear growth of multiplicity with energy,

$$
N \sim \frac{E}{E^{\bullet}},
$$

where $E^{*}$ is the threshold energy for producing a single particle per collision (see figure 2.5.2( $\mathrm{a})$ )).

The DEM suppresses this unacceptable growth with energy by considering a diffractive process. In order for such a diffractive scatter to occur, the scattered state must have a lifetime long compared to the transit time of the nucleus, and this necessary formation time suppresses the cross section for secondary interactions. (This is a formation time argument, as discussed above).

In the DEM, the incident particle and the nucleus are excited to states with the same quantum numbers (excluding spin and parity) and more or
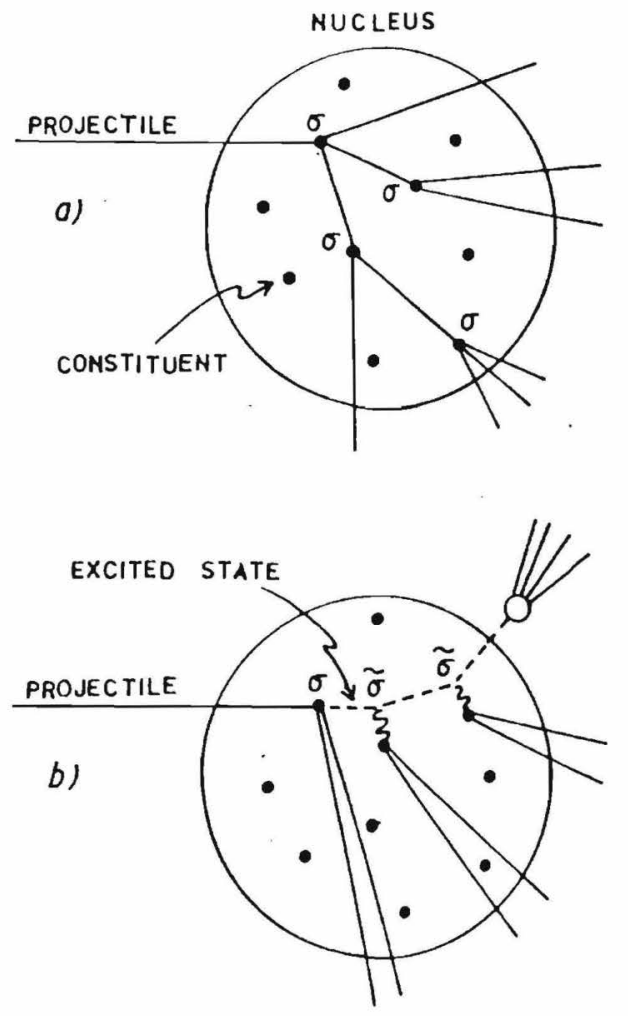

FIGURE 2.5.8.

Nuclear reinteractions for the Cascade and Diffractive Excitation Models. (a) Cascade Model picture. (b) Diffractive Excitation Model picture. 
less the same momentum. We expect that the important excitations will be of low mass, and if these excitations have lifetimes comparable to observed resonances, time dilation in the lab frame will make the beam excited state stable while it traverses the nucleus. Each nuclear excitation decays within the nucleus, but they are assumed not to have sufficient energy to produce further secondary interactions, since the beam excitation carries away most of the energy (in short, the beam excitation undergoes a chain of "diffractive"-type collisions, see figure 2.5.2(b)).

The DEM results in an $R(\nu)$ of the form

$$
R(\nu)=\frac{1}{2}\left(\nu+\frac{2}{1-p_{2}}\right)-\left(1+p_{2}\right) \frac{\nu}{4(N(E))} .
$$

In eqtn. 2.5.7 $p_{2}$ is the probability of exciting two beam diffractive states instead of one, and $N(E)$ is the characteristic multiplicity at energy $E$. Usually $p_{2}$ is taken as $0.1 \leq p_{2} \leq 0.5$.

The Two-Fireball Model (TFM) is a particular instance of DEMtype models (the excited states are refered to as "fireballs"). Because the beam fireball's decay to mesons is not a very sensitive function of its degree of excitation, one estimates that this fireball's contribution to the multiplicity will be $\frac{1}{2}\left(N_{h-n u c i e o n}\right)$, and each target fireball excited contributes roughly the same (there are $\nu$ target fireballs). Then we have

$$
R(\nu)=\frac{1}{2}(1+\nu)
$$

in the TFM.
C. The Energy Fiux Cascade Model.

The Energy Flux Cascade Model (EFC) ${ }^{|41|}$ bears a strong resemblance to the LHD in that it considers the most important characteristics of the nuclear interaction process to be described by following the evolution of the stress-energy tensor and ignores constituent subprocesses. Only the net fux of energy through the nucleus is considered important.

The EFC considers the question: If one evolves the final multi-particle state back wards into a compressed state, how does the energy flux proceed? The EFC uses a classical picture because it is constructed in rapidity space, and it assumes that only very short range correlations in rapidity are relevant. In the EFC, particles will be formed when the rapidityevolution equation indicates a rapidity width comparable to a typical hadronic width has been reached; whereas in the LHD particles were formed when the fluid cooled to an energy density comparable to that of free hadrons, and were broken up according to Bose-Einstein statistics.

All calculations are done in rapidity space using the light cone variables $x_{ \pm} \equiv t \pm z$ ( $z$ is along the beam axis and transverse motion is ignored). By noting that $(E-p) /(E+p)=(t-z) /(t+z)$, we find the free-particle trajectories to be

$$
\frac{x_{-}}{x_{+}}=\exp \left[(-2)\left(\frac{1}{2}\right) \ln \frac{E+p}{E-p}\right]=e^{-2 y} .
$$

The characteristic distance in which we are interested is one mean free path $\lambda$ (note that $x_{+}=2 \lambda$ ) 
In the EFC we slice the energy flux so that each slice has a thickness appropriate to the hadronic width. Let $y_{\max }$ be the maximum rapidity in the lab frame and $y_{\min }$ the minimum in that frame. Then if $y_{1}$ is the rapidity where we take the leading slice we should consider the appropriate width for this slice of average rapidity $\bar{y}=\left(y_{1}+y_{\max }\right) / 2$. Let $T_{0}$ be the rest frame thickness of a hadron. The width in $x_{-}\left(\Delta x_{-}\right)$will be $\mathbf{4} T_{0}$. Then from the fact that $x_{-}$transforms as

$$
\begin{aligned}
x_{-}^{\prime} & =\gamma\left(x_{-}+\beta x_{-}\right) \\
& \approx 2 \gamma x_{-}
\end{aligned}
$$

and the relation of the rapidity to the rest frame $(y=0)$

$$
y=-\ln [\gamma(1+\beta)]
$$

$$
\approx-\ln 2 \gamma
$$

from which we see that $\gamma \approx \frac{1}{2} e^{-\eta}$, we have

$$
\left.\Delta x_{-}\right|_{0}=4 T_{0} e^{-1}
$$

(terms of the order $1 / \gamma$ have been ignored throughout).

Equating the $\Delta x_{-}$of eqtn. 2.5.12 to the $\Delta x_{-}$of the evolution equations:

$$
\Delta x_{-}=\left|\left(x_{-}\right)_{b a c k}-\left(x_{-}\right)_{\text {front }}\right|
$$

$$
=e^{-2 y_{1}} x_{+} \text {, }
$$

(where 2.5.13 follows because $\left(x_{-}\right)_{\text {front }}$ is on the light cone), we have

$$
y_{1}=\frac{1}{3} y_{\max }+\frac{2}{3} \ln \left(\frac{x_{+}}{4 T_{0}}\right)
$$

Because the characteristic distance $\lambda \approx T_{0}$ so that $x_{+} \approx 2 T_{0}$, we can ignore the logarithm term in eqtn 2.5.14 and obtain

$$
y_{1} \approx \frac{1}{3} y_{\max }
$$

We see that the rapidity breakup is split $2 / 3$ to $1 / 3$ at each interaction, with a corresponding breakup into multiplicity of $2 / 3$ to $1 / 3$. Assuming that each " $1 / 3$ " slice will not have enough energy to reinteract, every rapidity "chunk" contributing to the breakup adds $\frac{1}{3}\left(N_{\text {nueleon }}\right)$ to the multiplicity, resulting in a $R(\nu)$ of the form

$$
R(\nu)=1+\frac{1}{3}(\nu-1) .
$$

If we had chosen a different breakup in rapidity space, then the $R(\nu)$ would have been parameterized by $\eta$ :

$$
R(\nu)=1+\eta(\nu-1) \text { where } \quad \eta \leq \frac{1}{2}
$$

\section{The Coherent (Collective) Tube Model.}

Models like the Landau Hydrodynamical Model are "collective" in nature. They describe a nuclear interaction in terms of some collective response of some portion of the nucleus; for the LHD, the collective response is that of a perfect relativistic fluid corresponding to $\nu$ nucleons. The extreme generalization of such collective models is the Coherent (or Collective) Tube Model, the CTM ${ }^{|42|}$. The CTM assumes that in a nuclear interaction a "tube" of $\nu$ nucleons recoils collectively from the interaction (see figure 2.5.3), and the remaining nucleons are spectators with no part in the interaction. At energies high enough to ignore (mass) ${ }^{2}$ terms 


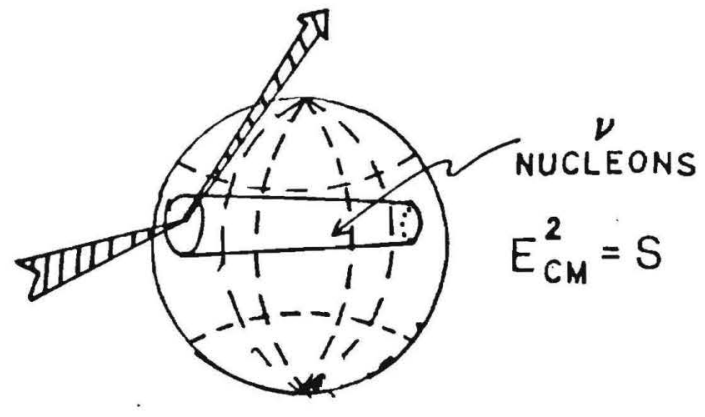

scales to

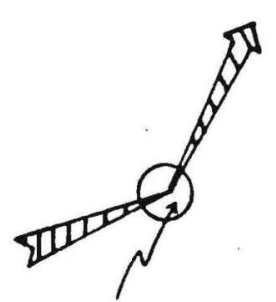

$E_{C M}^{\prime 2}=S_{C T M}$

$=\nu \mathrm{S}$

relative to the momenta involved, the square of the hadronic CM energy is $s \approx 2 M p_{\text {lab }}$, with $M$ the mass of the target and plab the laboratory momentum. Thus a "collective tube" interaction of $\nu$ nucleons of mass $M_{N}$ occurs at an "effective" of

$$
{ }^{-C T M}=2\left(\nu M_{N}\right) p_{l a b}=\nu \theta .
$$

In addition the CTM assumes a rigorous scaling law or universality principle, namely that in the center of mass system this "tube" collision has exactly the same characteristics as a collision with a nucleon at an equivalent center of mass energy $\sqrt{{ }^{\circ} C T M}$ (this universality is assumed to hold only for quantities that are independent of the quantum numbers of the colliding particles).

Using the two CTM assumptions, we see that any distribution independent of the quantum numbers in, for example, $h$-nucleus, can be described as a sum over $h$-nucleon distributions evaluated at ${ }^{8} C T M$. We sum over the probability of finding $\nu$ nucleons in the interaction tube $(P(\nu))$ to obtain:

$$
\rho_{h A}(s, \theta(s), \ldots)=\sum_{\nu=1}^{A} P(\nu) \rho_{h N}\left(s_{C T M}, \theta\left(s_{C T M}\right), \ldots\right) .
$$

$P(\nu)$ can in turn be calculated from first-principle assumptions about the nucleus; for example, a Woods-Saxon nuclear density, an independent particle model of nuclear structure, or a constant density approximation. (Note: $\boldsymbol{P}(\nu)$ is derived for the independent particle model in appendix A).

The CTM prediction for an average multiplicity from a nuclear target

FIGURE 8.5.8.

in terms of the same multiplicity on a nucleon target is

$$
\langle n(a)\rangle_{A}=\sum_{\nu=1}^{A} P(\nu)\left(n\left(a_{C T} M\right)\right\rangle_{N} .
$$


Because multiplicities in $h \mathrm{~N}$ can be roughly described by a power law growth with CM energy, ie $\langle n(s)\rangle_{k N}=\left(\delta / s_{0}\right)^{\alpha}\left(n\left(s_{0}\right) h_{h N}\right.$, this leads to the prediction

$$
\langle n(s)\rangle_{A}=\sum_{\nu=1}^{A} P(\nu)\left(\frac{s^{A} C T M}{s}\right)^{\alpha}\langle n(s)\rangle_{N} .
$$

Because of 2.5.18 this implies

$$
\langle n(s)\rangle_{A}=\langle n(s)\rangle_{N} \sum_{\nu=1}^{A} P(\nu) \nu^{\alpha} \equiv\langle n(s)\rangle_{N}\left\langle\nu^{\alpha}\right\rangle_{A},
$$

which leads immediately to

$$
R(\nu) \equiv \frac{\langle n(s)\rangle_{A}}{\langle n(s)\rangle_{N}}=\left\langle\nu^{\alpha}\right\rangle_{A}
$$

where $a$ is the exponent of the power law fit $\langle n\rangle_{N}=n_{0} 8^{\alpha}$.

If we let $\rho(s, \theta)$ be the inclusive rapidity distribution (that is, $\theta=y$ ) in eqtn. 2.5.19, so $\rho=\left(1 / \sigma^{i n e l}\right) d \sigma / d y$, then the CTM predicts that

$$
\frac{1}{\sigma_{A}^{\text {inel }}} \frac{d \sigma_{A}}{d y}=\sum_{\nu=1}^{A} P(\nu) \frac{1}{\sigma_{N}^{\text {inel }}} \frac{d \sigma_{N}}{d y}\left(\nu 8, y+\frac{1}{2} \ln \nu\right),
$$

where the $\frac{1}{2} \ln \nu$ derives from the rapidity shift between different CM frames:

$$
v^{\prime} \sim \ln \left[\sqrt{\frac{8}{s_{0}}}\left(E+p_{\|}\right)\right]=\ln \left(E+p_{\|}\right)+\ln \sqrt{\nu} \sim y+\frac{1}{2} \ln \nu .
$$

Perhaps the most impressive feature of the CTM is its unreasonable effectiveness. Unreasonable because quantum mechanically we expect collective reactions only at low momentum transfer because the relevant wavelength must be greater than the target size: If $\Delta x \sim$ a few fermis, then at most $\Delta p \sim$ a few hundred $\mathrm{MeV} / \mathrm{c}$. Experimentally we know that the bulk of interactions are above this range of momentum transfers. Furthermore, it seems unreasonable to expect such a simple sum of nucleon distributions to reproduce the vastly more complicated scenario of figure 2.5.1. In short, it is very hard to imagine reconstructing a CTMtype picture from the quark-parton picture. Considering these fundamental drawbacks, the CTM does a remarkably good job of describing nuclear interactions.

\section{E. Quark Models and Chains.}

Current nuclear interaction models using quarks and chains are also largely phenomenological models $|48|$. These models generally attempt to describe nuclear scattering in terms of weighted sums over structure functions, fragmentation functions, etc., of quarks in nucleon scattering. As previously discussed, quarks participating in an interaction form "strings" of color flux between colored products of the interaction, and these strings form multi-quark hadrons via $q \bar{q}$ production. These "strings" of hadrons are referred to as "chains" (the 'linking' color flux is broken by 'nodes' of pair production). By associating an average multiplicity, a rapidity density, or other measurable quantity with a chain, nuclear scattering properties can be described by taking sums over all possible chain configurations, weighted by the probability of that chain configuration.

Consider for example the model of Capella and Tran Thank Van for $p A$ scattering (herein called the CTTV model ${ }^{|41|}$ ). As shown in figure 


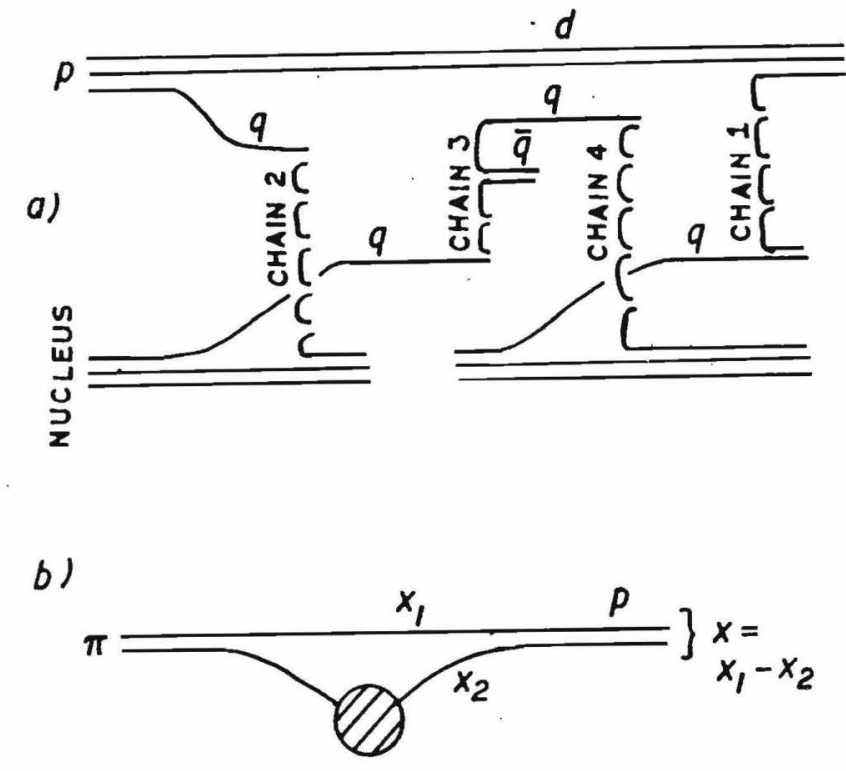

FIGURE 8.5.4.

Quark model picture of nuclear interaction. (a) A chain model pieture of p nucleus scattering (see text). (b) Momentum fractions for a leading quark (see text). 2.5.4(a), there are four possible types of chain: Chains 1 and 2, which are equivalent to the chains produced in $p p$ scattering, between ralence quarks and valence diquarks; Chain 4, between a sea quark and a valence diquark; And chain 3, between a sea quark and sea antiquark. Assuming that each chain fragments in the same way for nuclear and nucleon cases, and that the quark momentum fraction distributions are also the same, we can immediately write down the nuclear scattering results in terms of a sum over these chains.

Chains 1 and 2 appear only once in a nuclear interaction; if there are $\nu$ scatters total, there must be $\nu-1$ occurances of both chains 3 and 4 . Letting $N_{i}(y), i=1 \ldots 4$, be the rapidity density $(1 / \sigma) d \sigma / d y$, and $\sigma_{\nu}$ the cross section for $\nu$ collisions in nucleus $A$, we have

$N^{\dot{p} A}(y)=\frac{1}{\sigma^{p A}} \frac{d \sigma^{p A}}{d y}=\sum_{\nu=1}^{A} \frac{\sigma_{\nu}}{\sigma^{p A}}\left[N_{1}(y)+N_{2}(y)+(\nu-1)\left(N_{8}(y)+N_{4}(y)\right)\right]$.

If we assume that the $N_{i}(y)$ are independent of $\nu$ (a good approximation except for a slight $\nu$ dependence of $N_{1}$ coming from 4-momentum conservation) and use

$$
\sum_{\nu=1}^{A} \nu \sigma_{\nu} \equiv(\nu) \sigma^{p A}=A \sigma^{p p}
$$

(see appendix A for the expression for $(\nu)$ used in relation 2.5.27), we have

$$
N^{p A}(y)=N_{1}(y)+N_{2}(y)+((\nu\rangle-1)\left(N_{3}(y)+N_{4}(y)\right) .
$$

The $N_{i}(y)$ can then be written down in terms of integrals over quark structure functions and fragmentation functions.

As a simple approximation in the CTTV model, note that the universality assumption indicates that in terms of average multiplicities, given 
that $N_{1}$ and $N_{2}$ are the two chains occuring in $p p$ scattering,

$$
\langle N\rangle_{1}=\langle N\rangle_{2}=\frac{1}{2}\langle N\rangle_{p p}
$$

Taking the simple approximation that sea and valence quark momentum fraction distributions are equivalent, we have $\langle N\rangle_{4}=\langle N\rangle_{2}$. Thus

$$
R(\nu)=\frac{\langle N\rangle_{p A}}{\langle N\rangle_{p p}}=1+\frac{1}{2}(\nu-1)+O\left(\frac{\langle N\rangle_{s}}{(N)_{p p}}\right),
$$

which, in the limit that $O\left(N_{3}\right) \ll O\left(N_{1}\right), O\left(N_{2}\right), O\left(N_{4}\right)$ (equivalent to assuming that at experimental energies a $q \bar{q}$ chain has low invariant mass compared to a $q d$ chain), recovers the familiar $R(\nu)=\frac{1}{2}+\frac{1}{2} \nu$.

Note that strongly forward particles (particles with $x_{F} \approx 1$ ) will contain the remnant quark in figure 2.5.1(b), and these forward particles' properties will depend only on the momentum fraction of the remnant quark $\left(x_{1}\right)$. To see this, let $x_{2}$ be the momentum fraction of the quark picked up to hadronize the remnant quark (see figure 2.5.4(b)), and $x$ be that of the forward meson $\left(x=x_{1}-x_{2}\right)$. The momentum of the forward meson $p$ will be $p \approx x_{1} p_{1}+x_{2} p_{2}$, with $p_{1}$ the momentum of the incident meson and $p_{2}$ the momentum of the target nucleon. Then

$p^{2}=m^{2} \approx x_{1}^{2} p_{1}^{2}+x_{2}^{2} p_{2}^{2}+2 x_{1} x_{2} p_{1} \cdot p_{2} \approx x_{1} x_{2}\left(2 p_{1} \cdot p_{2}\right) \approx x_{1} x_{2} \theta(2.5 .31)$

(where second-order masses have been ignored throughout) so that

$$
x_{1} x_{2}=\left(x+x_{2}\right) x_{2}=\frac{m^{2}}{g} .
$$

(the definition of $x$ has been used in 2.5.32). From 2.5.32 we see that for large $s\left(\right.$ ie $\left.\mathrm{m}^{2} / \mathrm{s} \rightarrow 0\right)$ and high $x$ of the produced meson, $x_{2} \rightarrow 0$ and the forward meson's properties depend only on the projectile ${ }^{[45]}$. 


\section{Notes for Chapter 2}

1. Democritus, b.c. 460BC - d.c. 370BC. Evidently a wealthy citizen of Thrace, he posited that all physical phenomens are produced by compounds of atoms, "indivisibles" which are fundamentally the same, thereby foreshadowing the modern picture of elementary particles by roughly 25 centuries.

2. Antoine-Laurent Lavosier, French chemist, carefully set out the concept of elements as the fundamental chemical constituents in "Traité élémentaire de chemien in 1789. Dmitri Ivanovich Mendeleyev laid out the principles of the periodic table in the late 1860s - early 1870s.

3. E. Rutherford, Phil. Mag. 21, 668 (1911); H. Geiger and E. Marsden, Proc. Roy. Soc. A82, 495 (1909), Phil. Mag. 25, 604 (1913)

4. C.D. Anderson and S. Neddermeyer, Phys. Rev. 23, 884 (1937); Phys. Rev. 54, 88 (1938)

5. C.M.G. Lattes et al., Nature 159, 885 (1947)

6. F. Reines and C. Cowan, Phys. Rev. 113, 273 (1959)

7. see M. Roos et al., "Review of Particle Properties", LBL-100 Revised (UC-34d), which currently runs to 294 pages

8. see J.D. Bjorken and S.D. Drell, "Relativistic Quantum Mechanics" and "Relativistic Quantum Fields", McGraw Hill (1964-1985)

9. J.G. Bronson, "Proceedings 1981 International Symposium on Lepton and Photon Interactions at High Energies," edited by W. Pfeil, 279 (Bonn, 1981)

10. see reference 3
11. A. Bodek et al., Phys. Rev. Lett. 30, 1087 (1973); G. Miller et al., Phys. Rev. D5, 528 (1972)

12. M. Gell-Mann, Phys. Lett. 8, 214 (1964)

13. $\quad$ see section 2.2

14. J.J. Aubert et al., Phys. Rev. Lett. 33, 1404 (1974); J.E. Augustin et al., Phys. Rev. Lett. 33, 1408 (1974)

15. S.W. Herb et al., Phys. Rev. Lett. 39, 252 (1977); J. Ellis et al., Nucl Phys. B131, 285 (1977)

18. M.L. Perl et al., Phys. Rev. Lett. 35, 1489 (1975); Phys. Rev. Lett. 38, 117 (1978)

17. The $W$ and $Z$ bozons have only recently been observed. See $M$. Banner et al., Phys. Lett. 122B, 476 (1983); G. Arnison et al, Phys. Lett. 126B, 398 (1983)

18. P.W. Higgs, Phys. Lett. 12, 132 (1964); Phys. Rev. Lett. 13, 508 (1964); Phys. Rev. 145, 1156 (1968)

19. see D.N. Schramm, Physics Today 36, 27 (A pril 1983), and references therein

20. H.D. Politzer, Phys. Rev. Lett. 30, 1346 (1973); D. Gross and F. Wilczek, ibid, 1343

21. N. Cabibbo, Phys. Lett. 10, 513 (1963); S.L. Glashow, J. Iliopoulus, and L. Maiani, Phys. Rev. D2, 1258 (1970)

22. Much of the discussion in this section follows E.S. Abers and B.W. Lee, Phys. Rep. 9, no. 1, 1 (1973); and T.D. Lee, "Particle Physics and Introduction to Field Theory", Harwood Academic Publishers (1981). The book by T.D. Lee is an invaluable tool for understanding modern principles of gauge theory and is atrongly recommended by the author.

23. S. Weinberg, Phys. Rev. Lett. 19, 1284 (1967) 
24. A. Salam and J.C. Ward, Nuo. Cim. 11, 568 (1959); Phys. Lett. $13,168(1964)$

25. F. Niebergall, invited talk at Neutrino ' 82 Conference, Balatonfüred Hungary (1982)

28. see reference 17

27. E. Fermi, Z. für Physik 88, 181 (1934)

28. W. Marciano and H. Pagels, Phys. Rep. 36, 137 (1978)

29. see "Proceedings of the 1979 International Symposium on Lepton and Photon Interactions at High Energies", edited by T.B.W. Kirk and H.D.I. Abarbanel, Fermilab, Ill.

30. This discussion of quark confinement follows that given in T.D. Lee, "A Festschrift for Maurice Goldhaber", edited by G. Feinberg, A.W. Sunyar, and J. Weneser (New York, New York Academy of Sciences, 1980)

31. D.J. Gross and F. Wilczek, Phys. Rev. Lett. 30, 1343 (1973); H.D. Politzer, ibid, 1346

32. J. Ambjørn, P. Olesen, and C. Peterson, LU-TP-84-5 (March 1984)

33. $\quad$ see reference 21

34. M. Kobayashi and K. Maskawa, Progr. Theor. Phys. 49, 652 (1973)

35. This discussion follows T.D. Lee, reference 22

38. C.G. Callan and D.G. Gross, Phys. Rev. Lett. 22, 158 (1969)

37. $\quad$ see reference 7

38. A.K. Wroblewski, "Topologic Cross Sections in $\pi p$ Interactions", Univ. of Washington, Visual Techniques Laboratory, Physics Note PHY-90 (1982)
39. L.D. Landau, "Collected Papers of L.D. Landau" (New York, New York 1988); S.Z. Belenkij and L.D. Landau, Suppl. Nuov. Cim. 3 15 (1956); see also B. Andersson, Nuov. Cim. 38A, 329 (1977)

40. see, for example, A. Dar and J. Vary, Phys. Rev. DQ, 2412 (1972)

41. K. Gott fried, "High-Energy Physics and Nuclear Structure”, edited by Gunnar Tibell (Uppsala, Sweden, 1973); B. Andersson, Nucl. Phys. B95, 237 (1975)

42. G. Berlad, A. Dar, and G. Eilam, Phys. Rev. D13, 161 (1978); Y. Afek, G. Berlad, A. Dar, and G. Eilam, TECHNION-PH-76-87 (1978); S.A. Azimor et al., Phys. Lett. 73B, 339 (1978)

43. Some models take a more fundamental approach but have low predictive power. These "soft field theory" models are not discussed here; but see, for example, E.S. Lehman and G.A. Winbow, cussed here; but see, for exam
Phys. Rev. D10, 2962 (1974)

44. A. Capella and J. Tran Thank Van, LPTPE-80/7 (1980)

45. Bee A. Dar and Fujio Takagi, Phys. Rev. Lett. 44, 788 (1980) 


\section{CHAPTER \&}

\section{EXPERIMENT E540}

5 8.1. The experiment run, apparatus, and event reconstruction. Experiment E546 ${ }^{[1]}$ consisted of a high energy mixed $\nu$ and $\nu$ beam incident on a Neon- $\mathrm{H}_{2}$ mixture, and was conducted at Fermilab, a U.S. national laboratory. The experiment was designed to search for dimuon production in $\nu \mathrm{Ne}$ (which is a signature of charm production in weak interactions), and to generate a large sample of high- $\left\langle Q^{2}\right\rangle$ neutrino data to study deep-inelastic $\nu$ interactions in a regime where perturbative techniques of gauge theory are more likely to apply.

The experiment E546 run took place from September 1977 to January 1978. The neutrino beam was generated using the Quadrupole-Triplet neutrino beam line. $400 \mathrm{GeV} / \mathrm{c}$ protons were extracted from the Fermilab main ring and directed onto an alumina target, with a total of about
$10^{12}-10^{18}$ protons per pulse in a $2 \mathrm{msec}$ spill. Resulting secondaries (mainly $\pi s$ and $K \mathrm{~s}$ ) were focused using a quadrupole train tuned for an optimum of $200 \mathrm{GeV} / \mathrm{c} \pi^{+}$. Accepted $\pi s$ and $K \mathrm{~s}$ entered a 400 meter evacuated decay pipe where $(\bar{\nu})$ were produced from the decays $\pi \rightarrow \mu \nu$ and $K \rightarrow \mu \nu$. The decay pipe is followed by a 1 kilometer earth and rubble berm which absorbed all secondary particles except neutrinos, resulting in the final neutrino beam.

Due to the wide range of generating $\pi$ and $K$ energies, the neutrino beam contains a wide range of neutrino energies. The momentum content of the final neutrino beam, as calculated from the current of the $\pi$ and $K$ decay beam, is shown in flgure 3.1.1. The average neutrino energy was $\left\langle E_{\nu}\right\rangle=90 \mathrm{Gev}$, and the ratio of $\nu$ to $\bar{\nu}$ induced events was approximately 6:1.

The target for the experiment was the 15-foot Fermilab Bubble Chamber (FBC), filled with a $\mathrm{Ne}-\mathrm{H}_{2}$ mixture. This mixture was $47 \%$ (atomic) Ne at a temperature of $29.5^{\circ} \mathrm{K}$ and a density of $0.56 \mathrm{gm} / \mathrm{cm}^{3}$. Such a mixture provides a radiation length of $53 \mathrm{~cm}$ and a pion interaction length of approximately $193 \mathrm{~cm}$. A $3.0 \mathrm{~T}$ magnetic field momentum analyzed charged particles in the chamber. The observed rate of $\left(-\bar{\nu}^{\prime} \mathrm{Ne}\right.$ interactions in the FBC was about 1 per 10 to 15 accelerator cycles.

Five separate physics laboratories participated in experiment E546. These laboratories were: Department of Physics and Lawrence Berekeley Laboratory, University of California, Berkeley, California; Fermi National Accelerator Laboratory, Batavia, Illinois; Department of Physics, University of Hawaii at Manoa, Honolulu, Hawaii; Visual Techniques Laboratory Department of Physics, University of Washington, Seattle, WA; and De- 


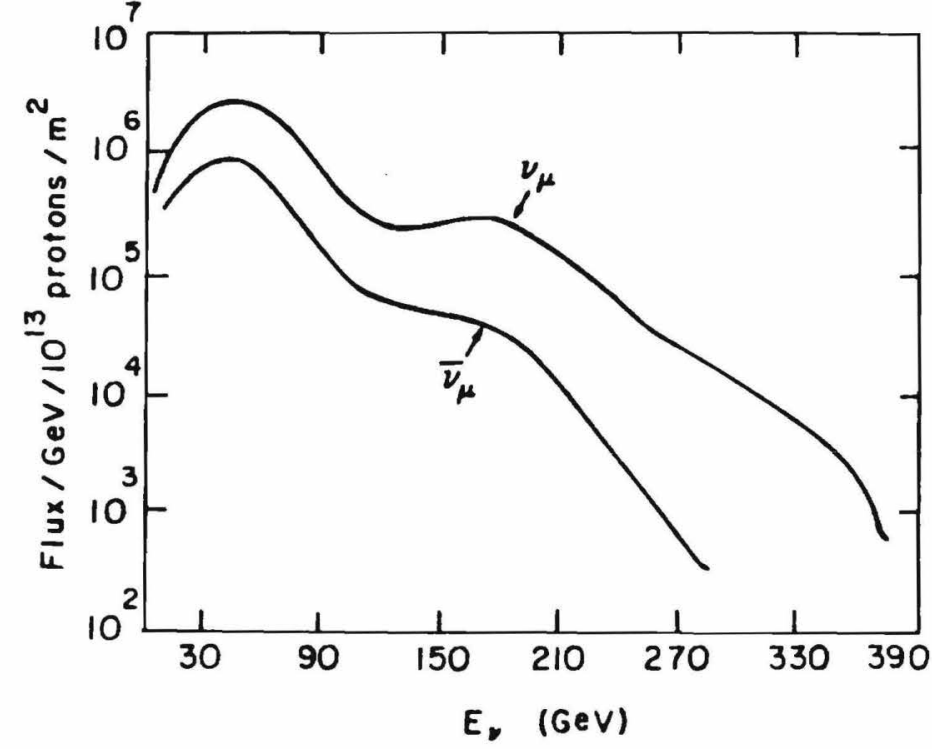

FIGURE 8.1.1.

partment of Physics, University of Wisconsin, Madison, Wisconsin. All five participated in both scanning and measuring phases of data reduction.

A total of some 328000 pictures were obtained, and all pictures were scanned by the collaboration. Candidate charged current neutrino interactions were required to occur in a central fiducial volume of $\mathbf{1 4 . 5}$ $\mathrm{m}^{3}$, have no incoming track, and to have at least three outgoing tracks, of which at least one (the $\mu$ candidate) had to leave the chamber through the exit wall with no visible kinks, interactions, or obvious energy loss. All candidate neutrino interactions had all their $\mu$ candidate tracks measured; the results of the $\mu$ candidate measurements were extrapolated from the FBC and compared with data from the External Muon Identifler (EMI) to determine if the $\mu$ candidate met the muon acceptance criteria.

The External Muon Identifier (EMI) consisted of two planes of Proportional Wire Chambers (PWCa) downstream of the FBC (see figure 3.1.2). Zinc absorber separates the first plane from the FBC, and lead absorber between the first and second planes provides a total of between 7 to 11 hadron absorption lengths, depending on particle path, to the second plane. Since muons do not undergo strong interactions and lose energy only through ionization ( $d E / d x$ ) and multiple coulomb scattering, they easily penetrate this absorber and are recorded in the EMI PWCs.

Each PWC had about $1 \mathrm{~m}^{2}$ of active area. There were $18 \mathrm{PWCs}$ in the first plane and 21 in the second plane. Any charged particle traversing these PWCs produced an output voltage pulse; the location and time for each pulse was digitized and recorded, where the time clock was synchronized with the neutrino beam pulse, and a clock tick was 35.7 nsec. The measured electronic efficiency of each FMl plane for muons was 


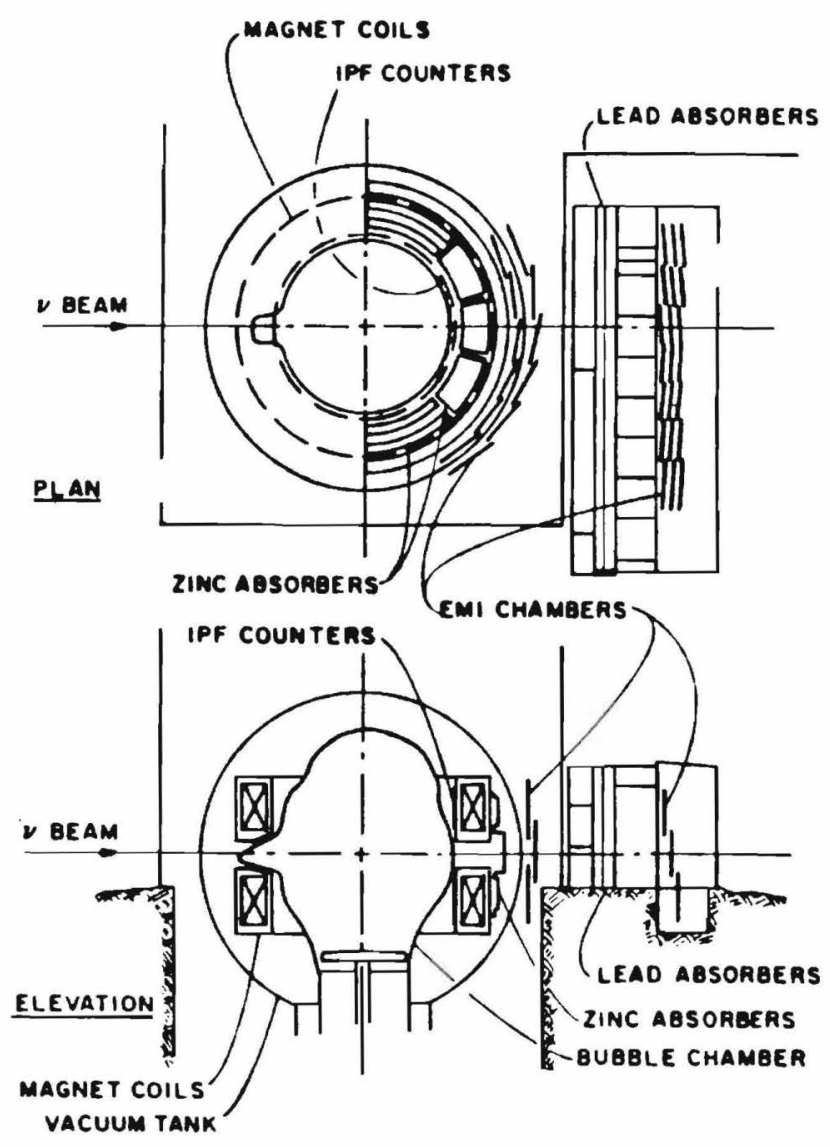

FIGURE 8.1.2.

EMI layout and bubble chamber.
$90 \%$, and the combined electronic and geometric efficiency for both planes was $73 \%$.

In order for a candidate $\mu$ to be classifled as a $\mu$, its extrapolation to the EMI had to generate a simultaneous hit in both planes (defined as within 10 clock ticks), had to satisfy a 3- or 4-constraint fit to the two planes, and the fit in turn had to have a two-plane EMI confidence level $C L \geq 10^{-4}$.

Candidate ${ }^{\left.(-)^{\prime}\right)}$ Ne interactions with a $\mu^{ \pm}$identifled by the EMI were taken as CC neutrino intersctions. All CC neutrino interactions were fully measured, including all neutrals decaying, converting, or interacting within two radiation lengths of the event vertex (the momentum of a neutral track was reconstructed from the momenta of its decay or conversion products). Measurements were done on film plane measurement devices. Tracks that did not develop suffeient curvature for a momentum determination before interacting had their momentum reconstructed from the measured momenta of all tracks from the secondary vertex.

The resulting fully measured $\mathrm{CC}$ neutrino sample used in this analysis consisted of: $\nu \mathrm{Ne}, 8479$ events; $\nu \mathrm{Ne}, 1384$ events.

\subsection{Neutrino energy correction.}

Because the incident neutrino in $\left.\mathbf{a}^{(} \bar{\nu}\right)$ Ne interaction is neutral there is no way to directly measure the incident $\left(\bar{\nu}^{\prime}\right)$ energy, as opposed to an experiment with a charged particle beam, such as WA51. Furthermore, as discussed in section 3.1 , the E546 neutrino beam covers a wide range of neutrino energies $E_{\nu}$. This spread in $E_{\nu}$ comes from the spread in 
energies of the $\pi^{ \pm}$and $K^{ \pm}$from which the neutrinos are produced. In turn this spread in the decay-beam momentum is necessary to generate an adequate neutrino flux in the neutrino beam, since the total cross section $\left.\operatorname{for}^{(} \bar{\nu}\right) N$ at $\mathbf{E} 546$ energies is only $\sim 10^{-0} \mathrm{mb}$.

While the overall momentum spectrum of the neutrino beam is known from the momentum profile of the decay-beam, the incident ${ }^{(} \bar{\nu}^{\prime}$ energy in any individual interaction must be determined from the propertie of the interaction itself. If all finsl state particles were detected and their total energy was contained in the detector, then we could simply "add up" the final state energy to find the neutrino energy. Unfortunately some neutrals are not detected in $\mathrm{E} 546$ ( VOs that do not decay in the bubble chamber or gammas that convert outside the gamma measurement radius), and so this fraction of the total energy will be lost and must be compensated for.

Techniques to compensate for this missing energy generally rely on the assumption that the undetected, or "missing", neutral energy will also unbalance the momentum transverse to the incident $(-\bar{\nu})$ direction (which is well known). Clearly if all final state particles are detected, then $\sum \overrightarrow{\mathbf{p}}_{T}=$ 0 . Once we have a relation between the total amount of missing transverse momentum ( $\left.p_{T}^{m i o s}=\left|\sum \overrightarrow{\mathbf{p}}_{T}\right|\right)$ and the amount of missing energy ( $\left.E^{\text {mioe }}\right)$, we can find the correct neutrino energy by $E_{\nu}=E^{\text {det }}+E^{\text {mies }}\left(p_{T}^{m i e s}\right)$, where $E^{\text {det }}$ is the total detected final state energy.

The simplest assumption to make is that the total neutral momentum axis $\overrightarrow{\mathbf{P}}_{\text {neus }}$ coincides with the charged momentum axis $\overrightarrow{\mathbf{P}}_{\text {charge }}$ of the hadronic system. In this case we can just scale the detected energy by $\left(1+p_{T}^{\text {mico }} / p_{T}^{\text {det }}\right)$. This is the " $p_{T}$ balance" technique ${ }^{|2|}$. However, if the neutral and charged axes are not parallel, then $p_{T}$ balance can yield un- reasonable results. To compensate for such difficulties, a modifled method was introduced by the Bonn group ${ }^{[3]}$

Large values of the correction scale factor $G_{p T b a l}=\left(1+p_{T}^{\text {mioe }} / p_{T}^{\text {det }}\right)$ tend to indicate a failure of the method. To compensate the Bonn group used the triangle inequality to limit the correction scale factor $G$ : instead of taking the actual $p_{t}^{\text {det }}=\left|\sum_{\text {hedrone }} \vec{p}_{T}^{\text {det }}\right|$, they used $p_{t}^{\text {det }}=$ $\sum_{\text {hadrona }}\left|\overrightarrow{\mathbf{p}}_{T}^{\text {det }}\right|$. This is the "Bonn method" of energy correction.

From this we see that any $p_{T}^{m i c o}$ based correction is inherently dependent on the topology of the event: for large topologies it is much more likely that $\mathbf{p}_{T}^{\text {neut }} \| \overrightarrow{\mathbf{p}}_{T}^{\text {eherge }}$ than for small topologies. Small topologies are inherently harder to correct for.

The LUND monte-carlo ${ }^{[4]}$ was used to study the relative efficacy of the two correction techniques in E546. The modified version of the montecarlo ${ }^{[5]}$ used has the option of smearing flnal state momenta according to the measured $\mathrm{E} 546$ error distribution as a function of $W^{2}$, and similarly of "failing" final state tracks according to E546 failure rates. In general the monte-carlo reproduces the E546 measurements well and, of course, gives the option of comparing "actual" and "corrected" energies.

The results of this study are summarized in table 3.2.1. The results point out the difference between theory and experiment. If all detected tracks (including neutral fits) are measured well, then $p_{T}$ balance must be accurate when averaged over a large sample of events, because in the large statistics limit the missing and detected axes are parallel. However, in the monte-carlo comparison, the Bonn method proves more accurate than the $p_{T}^{\text {bal }}$ method when track failures and momentum errors are taken into account. 


\section{TABLE 8.2.1.}

Comparison of Bonn and $p$ en energ correction techniques using the LUND monte-carlo.

\begin{tabular}{|c|c|c|c|c|c|c|}
\hline \multirow{2}{*}{$\begin{array}{c}\text { Tracks } \\
\text { Detected }\end{array}$} & \multicolumn{3}{|c|}{$E_{E^{P A L} / E^{A C T U A L}}^{B A}$} & \multicolumn{3}{|c|}{$E^{\text {BONN } / E^{\text {ACTUAL }}}$} \\
\hline & Mean & Width & Skewness & Mean & Width & Skewnes: \\
\hline 2 & 1.00 & 0.13 & 0.29 & 1.00 & 0.11 & -0.12 \\
\hline 3 & 1.02 & 0.12 & 0.18 & 1.02 & 0.11 & 0.24 \\
\hline 4 & 1.02 & 0.15 & 0.18 & 1.00 & 0.11 & -0.11 \\
\hline 5 & 1.03 & 0.16 & 0.15 & 1.01 & 0.12 & -0.20 \\
\hline 6 & 1.02 & 0.14 & 0.19 & 1.01 & 0.11 & -0.38 \\
\hline 7 & 1.04 & 0.15 & 0.19 & 1.02 & 0.10 & -0.24 \\
\hline 8 & 1.05 & 0.18 & 0.24 & 1.01 & 0.11 & -0.29 \\
\hline 9 & 1.06 & 0.19 & 0.16 & 1.03 & 0.11 & -0.11 \\
\hline
\end{tabular}

In the case of "unsmeared" events, $\left\langle E_{\nu}^{\text {phbal }}\right\rangle=\left\langle E_{\nu}^{\text {actual }}\right\rangle$, as expected. But in the realistic case of "smeared" events $\left\langle E_{\nu}^{B o n n}\right\rangle \approx\left\langle E_{\nu}^{\text {eotuel }}\right\rangle$, with $\left\langle E_{\nu}^{\text {Tbal }}\right\rangle$ about $35 \mathrm{MeV}$ higher than the actual value and $\left\langle E_{\nu}^{B o n n}\right\rangle$ only about $10 \mathrm{MeV}$ higher. Furthermore, the normalized Bonn energy distribution. width is about $1 / 2$ that of the normalized $p_{T}^{\text {bet }}$ energy distribution width, and as expected the Bonn distribution is skewed downward while the $p_{T}^{\text {bal }}$ distribution is skewed upward.

This change of the optimal technique from $p_{T}^{\text {bed }}$ to Bonn when track errors are added must come from momentum smearing and not track failures, since track failures only add to the $p_{T}^{m i s}$ value and do not change the relative efficiency of the two techniques. Most of the change will come from high momentum tracks with relatively higher measuring errors. Since the muon has a long measurement length and is constrained by the EMI, it is relatively a higher quality measurement than the hadronic tracks. We can thus consider $\mathbf{p}_{\mu}$ fixed. Then smearing the hadron tracks effectively moves $\overrightarrow{\mathbf{p}}^{\text {neut }}$ away from $\overrightarrow{\mathbf{p}}^{\text {charge }}$ and increases the relative effliency of the Bonn method.

Since the quantity actually measured is the currature $k$, the errors are Gaussian-distributed in $\boldsymbol{k}$ :

$$
P(\delta k) \sim \exp \left(-(\delta k)^{2} / 2\right),
$$

but the errors in the momentum $p=1 / k$ will go as

$$
P(\delta p) \sim \exp \left(-(\delta p)^{2} / 2 p^{4}\right)
$$

Thus the width of the error distribution in $p$ increases as $p$ increases, and in general tracks will have their momentum smeared upward over a finite p range. This favors the Bonn technique. 
The conclusion is that for data samples where the events meet some "quality criteria" indicating they are very well measured, then the $p_{T}^{b a l}$ technique is optimal; but for more general dats amples with significant errors, such as the general E54B sample, the Bonn technique is optimal.

In light of this, the Bonn energy correction technique was used for all calculations in this dissertation. To summarize:

$$
E_{\nu}=p_{L}^{\mu}+G_{B o n n} \cdot p_{L}^{\text {had }}
$$

where $p_{L}^{\mu}\left(p_{L}^{h a d}\right)$ is the component of the muon (hadronic) momentum parallel to the incident $(\bar{\nu})$ direction, ie the longitudinal component; and where

$$
G_{\text {Bonn }}=1+\frac{\left|\mathbf{p}_{T}^{\mu}+\sum_{i-h a d r a n d} \overrightarrow{\mathbf{p}}_{T}^{i}\right|}{\sum_{\text {i-hedrone }}\left|\overrightarrow{\mathbf{p}}_{T}^{i}\right|} .
$$

In addition, events with $G_{B o n n}>10$ were considered uncorrectable, and not used in this analysis.

\section{Notes for Chapter \&}

1. For a complete list of papers from Experiment E546 and a more detailed discussion of the experiment, see E.J. Wolin, "Neutra Strange Particle Production in High Energy Neutrino Interactions”, PhD Thesis, University of Washington, Seattle, Washington, USA (1984). See also J. Orthel, PhD Thesis, University of California at Berekely, USA (1979)

2. G. Myatt, CERN/EFCA/72-4 Vol. II p. 117 (1972)

3. H.B. Heilmann, Univ. of Bonn Internal Report WA21-INT-1 (1978)

4. T. Sjöstrand, LU-TP-82-3 (1982)

5. see reference 1 for more details 


\section{CHAPTER}

\section{EXPERIMENT WA51}

\subsection{The experiment run and event scanning.}

Experiment WA51 consisted of a momentum-separated pion beam incident on a Neon- $\mathrm{H}_{2}$ mixture, and was conducted at the Centre for European Nuclear Research (CERN). The experiment was designed with two purposes in mind: (1) to search for flnal state protons with momentum $\geq 1 \mathrm{GeV} / \mathrm{c}$ (which cannot be identified by ionization techniques because of the relativistic $d E / d x$ plateau) at energies where no prior data was available; and (2) to provide $\pi \mathrm{Ne}$ data under conditions as close as possible to existing $\left(\bar{\nu}^{\prime} \mathrm{Ne}\right.$ data, so that the two data samples could be compared. In particular, Yeager et al ${ }^{[1]}$ had reported such fast protons (termed $p_{\text {fast }}$ tracks in this dissertation) at a level of about $0.5 p_{\text {faot }}$ tracks per event for $\pi^{ \pm} \mathrm{Ne}$ at $10.5 \mathrm{GeV} / \mathrm{c}$ incident momentum, and it was desirable to see if such a large number of $p_{\text {fart }}$ tracks continued to higher CM energies. This large number of $p_{\text {fast }}$ tracks proved difficult to explain using current theoretical models.

The experiment WA51 run occured in April 1979, with another run in May 1979 to gather more data. The incident pion beam was generated using the S3 beam line from the SPS (Super Proton Synchrotron), from $200 \mathrm{GeV} / \mathrm{c}$ protons incident on a beryllium target. There were $0.5 \times 10^{11}$ protons per pulse on target for a spill of approximately $2 \mu \mathrm{s}$. The beam line was tuned to select either $\pi^{+}$or $\pi^{-}$at momenta of either $30 \mathrm{GeV} / \mathrm{c}$ or $64 \mathrm{GeV} / \mathrm{c}$, and approximately equal amounts of data were generated at each energy and sign. Upstream Cherenkor counters indicated that the incident pion beam had $\leqslant 5 \%$ total proton contamination.

- The target for the experiment was the Big European Bubble Chamber (BEBC), filled with a $\mathrm{Ne}-\mathrm{H}_{2}$ mixture. This mixture was $75 \%$ (molar) $\mathrm{Ne}$ at a temperature of $29.15^{\circ} \mathrm{K}$ and a density of $0.707 \mathrm{~g} / \mathrm{cm}^{3}$ at particle injection. Such a mixture provides a radiation length of $41 \mathrm{~cm}$ and a pion interaction length of approximately $120 \mathrm{~cm}$. A $3.50 \mathrm{~T}$ magnetic field momentum analyzed charged particles in the chamber. To facilitate scanning the $\mathrm{S3}$ beam line was tuned so that $\sim 2-4$ beam pions entered the bubble chamber per frame.

Experiment WA51 ran parasitically (that is, on alternating bubble chamber expansions) with experiment WA52 ${ }^{[2]}$, which was a neutrino beam dump experiment to be analy zed using BEBC.

Three separate physics laboratories participated in experiment WA51 These laboratories were: Visual Techniques Laboratory, Department of Physics, University of Washington, Seattle, WA, USA; Centre de Recher- 
ches Nucleaires et Université L. Pasteur, Strasbourg, France; and Institute of Experimental Physics, University of Warsaw and Institute of Nuclear Research, Warsaw, Poland. All three participated in the scanning phase of data reduction, but only the Seattle and Warsaw labs provided measurement data.

A total of some 36000 pictures were obtained, and all pictures were scanned by the collaboration. In the scan, legitimate beam tracks were required to have the correct sign for the type of incident pion, to traverse a minimum distance before any interaction occured, and to match a curvature template. Legitimate interactions had to be on a legitimate beam and to occur a minimum distance from the exit wall, so that the sign of the charge of all outgoing tracks could be determined. In addition, for the $64 \mathrm{GeV} / \mathrm{c}$ data one and only one legitimate beam track was required on a frame in order to minimize scan inefficiencies from the higher multiplicities and more extensive showers of these events.

The number of positive outgoing tracks $\left(N^{+}\right)$, the number of negative outgoing tracks $\left(N^{-}\right)$, the number of protons $\left(N_{p}\right)$, and number of tracks whose sign could not be decided $\left(N^{ \pm}\right)$- becsuse of close interactions, usually - were recorded for each event. In addition, in the Seattle scan the interaction vertex was measured to insure that the event was in the fiducial volume, and the number of shower tracks ( $N^{\text {ehower }} \equiv N^{+}+N^{-}+N^{ \pm}$) was entered independently to check for misentries.

"Proton" tracks were required to have a minimum length to exclude very small $\delta$-rays (electrons ejected from an atomic shell by interacting with a charged particle), which can form "blobs" resembling a short stub. A proton had to be 1.5 times minimum ionizing, with the beam track defined as minimum ionizing; had to have positive charge if the track had sufficient curvature to determine its charge; and had to stop in the bubble chamber. Special care was taken in the scan to identify protons, with physicists initially working with scanners to locate difficult protons, and with periodic rechecks for proton efficiency.

All electrons and positrons were excluded from the multiplicity counts. At $75 \%$ molar $\mathrm{Ne}$, essentially all $e^{+}\left(e^{-}\right)$have identifying signatures of spiralization, brehmstrahlung, annihilation, or any combination thereof, so there is no contamination from asymmetric Dalitz decays in this data.

Results of each collaboration group were checked against other groups for consistency. After problems were resolved, all three groups were consistent. The final total number of accepted interactions resulting from the scan for each type of incident beam were:

$\begin{array}{ll}\pi^{+} 30 \mathrm{GeV} / \mathrm{c}: & 3858 \text { events } \\ \pi^{-} 30 \mathrm{GeV} / \mathrm{c}: & 5729 \text { events } \\ \pi^{+} 64 \mathrm{GeV} / \mathrm{c}: & 1516 \text { events } \\ \pi^{-} 64 \mathrm{GeV} / \mathrm{c}: & 1450 \text { events. }\end{array}$

\subsection{Corrections to the sean data.}

After the final sample of accepted events was assembled, the dats were corrected for rarious effects. As noted in section 4.1, some events contain tracks whose charge could not be determined $\left(N^{ \pm}\right)$due to close interactions, and these tracks must be corrected for. Although nearly all interactions in the $\mathrm{Ne}-\mathrm{H}_{2}$ mixture will be on $\mathrm{Ne}$, a small percentage will be on $\mathrm{H}$ and such events should be statistically removed from the 
sample. Also prior experiments have shown that a few percent of $\pi$-nucleus interactions at this energy will be coherent events; that is, the reaction will be with the entire nucleus instead of a nucleon in the nucleus. In order to analyze reinteraction effects these coherent events should be statistically excluded.

To correct for $N^{ \pm}$tracks, which comprise about $3 \%$ of all charged tracks in the final sample, all events with no $N^{ \pm}$tracks were selected from the data sample. These events were broken down according to the type of incident pion $\left(\boldsymbol{\pi}_{\mathbf{3 0}}^{+}, \overline{\boldsymbol{\pi}_{\mathbf{3 0}}}, \boldsymbol{\pi}_{04}^{+}, \boldsymbol{\pi}_{04}^{-}\right)$and the number of shower tracks ( $N^{\text {ekower }}$ ) in the event. Because there are 4 types of incident pion, and given that the maximum number of shower tracks for any event in the entire sample is $N_{\max }^{\text {shower }}$, this results in $4 \cdot N_{\max }^{\text {ehower }}$ categories, called "shower categories". For each incident pion type and shower count the probability that any given shower track in that shower category was a positive track was computed as $\mathrm{P}^{+}=\mathrm{N}^{+} /\left(\mathrm{N}^{+}+\mathrm{N}^{-}\right)$, where $\mathrm{N}^{+}$is the total number of positive shower tracks in that shower category, and $\mathrm{N}^{-}$ is the total number of negative shower tracks in that shower category. If there were no events for that shower category with $N^{ \pm}=0$, then $P^{+}$ was assigned as $P^{+}=0.5$.

During analysis, any event with one or more $N^{ \pm}$tracks then had those tracks redistributed to the $N^{+}$and $N^{-}$classifications according to the probability $\boldsymbol{P}^{+}$that it was a positive track.

To correct for pion interactions taking place on Hydrogen instead of Ne, the topological cross section for $\pi^{ \pm} p \rightarrow X$ were determined for $30 \mathrm{GeV} / \mathrm{c}$ and $84 \mathrm{GeV} / \mathrm{c}$ from data in the published literature ${ }^{[3]}$. From the topological cross sections, the fraction of $\pi p$ events with $N^{\text {charge }}=$
$0,2,4,6,8,10$, etc., for each incident pion type was calculated. Corrections for more than 10 prongs were ignored since such corrections are at the 0.01 event level.

The resulting expected "fraction of $\pi p$ events" were:

\begin{tabular}{ccccccc} 
& $N^{c h}=0$ & $N^{c h}=2$ & $N^{c h}=4$ & $N^{c h}=6$ & $N^{c h}=8$ & $N^{c h}=10$ \\
\hline$\pi_{30}^{-}:$ & 0.007 & 0.134 & 0.196 & 0.325 & 0.215 & 0.091 \\
$\pi_{30}^{+}:$ & 0 & 0.159 & 0.382 & 0.290 & 0.128 & 0.036 \\
$\pi_{84}^{-}:$ & 0.009 & 0.148 & 0.281 & 0.276 & 0.167 & 0.076 \\
$\pi_{84}^{+}:$ & 0 & 0.099 & 0.224 & 0.233 & 0.164 & 0.099
\end{tabular}

These distributions are of course corrected from observed experimental distributions to meet total charge constraints in $\pi^{ \pm} p$. To correct the $\pi^{ \pm} \mathrm{Ne}$ data one should technically use either "raw data" distributions or smear the above distributions to correspond to observed distributions in $\pi^{ \pm} \mathrm{Ne}$. However, since the total $\pi^{ \pm} p$ correction is of order $5 \%$ for all events, the maximum shift in any fractional category would be $\sim 0.75 \%$. Since the WA51 data itself has a statistical inaccuracy of a few percent, it seems unreasonable to perform such smearing. Also, such a smearing correction can not be done with any assurance of accuracy. For example, if we decide that $5 \%$ of the $N^{\text {ch }}=4$ events should be moved to the $N^{c h}=3$ category, then since this " $5 \%$ " depends on many arbitrary factors such as scanning efficiency, mixture density, and very close rescatters, the statistical error goes as $1 / \sqrt{N_{m p}\left(N^{c h}=4\right)} \gg 5 \%$ for experiment WA51. In light of this, the above "unsmeared" distributions were used for the $\mathbf{H}$ correction.

The total inelastic cross section ${ }^{|8|}$ for all beam types in WA51 for $\pi^{ \pm} p$ is $21 \pm 0.5 \mathrm{mb}$. In addition there is about $3 \mathrm{mb}$ of $\pi^{ \pm} p$ elastic cross 
section, of which about $1 \mathrm{mb}$ might effect the $\pi^{ \pm} p$ correction by producing a visible 2 prong event. Using the measured total cross section for $\pi^{ \pm} \mathrm{Ne}$ at these energies ${ }^{[4]} \sigma_{N a}^{T O T}=270 \pm 10 \mathrm{mb}$, a value for $\pi^{ \pm} p$ of $22 \mathrm{mb}$, and the BEBC WA51 experimental run mixture of 0.75 molar $\mathrm{Ne}=\mathbf{0 . 8 0}$ atomic $\mathrm{Ne}, 5.2 \%$ of all interactions are $\pi^{ \pm} p$ interactions.

In correcting the WA51 distributions, which are functions of the number of identified protons $\left(N_{p}\right)$, it is necessary to know how many $\pi^{ \pm} p$ interactions in WA51 will have an identifled proton. A simple physical argument suggests that about $1 / 3$ of all $\pi^{ \pm} p$ interactions will have an identified proton at these energies ${ }^{[5]}$.

We can check this with a simple calculation. At $30 \mathrm{GeV} / \mathrm{c}$ in WA51, $\left\langle N^{c h}\right\rangle \approx 6$, so $E^{l a b} /\left(N^{c h}\right\rangle \approx 5.2$. If we assume that the proton has $\left\langle E^{P}\right\rangle=5.2$ in the lab and that the distribution in $E^{P} \sim \exp \left(-E^{P} l\right)$, where $b$ is some inverse scale length, then setting

$$
\left\langle E^{p}\right\rangle=\int_{0}^{\infty} E^{p} e^{-E^{p} l} d E^{p}=5.2
$$

implies that $\boldsymbol{l}=\mathbf{0 . 4 4}$. Assuming the proton is "identified" if $\left|\overrightarrow{\mathbf{p}}_{\mathbf{p}}\right|<800$ $\mathrm{MeV} / \mathrm{c}$, which implies that $E_{\max }^{p} \approx 1.2 \mathrm{GeV}$, then the fraction of the distribution that has $E^{p}<E_{\max }^{p}$ is

$$
\begin{aligned}
f_{\text {oio p }} & =\int_{0}^{E_{\text {max }}^{p}} e^{-E^{p l}} d E^{p} / \int_{0}^{\infty} e^{-E^{p} 1} d E^{p} \\
& =0.41,
\end{aligned}
$$

which is indeed of the right order. Repeating this calculation at $64 \mathrm{GeV} / \mathrm{c}$, where $\left\langle N^{c h}\right\rangle \approx 8$, yields $f_{\text {viop }}=0.34$. Therefore the value of $f_{\text {oiop }}$ used was $1 / 3$
Given these values, the WA51 distributions are corrected for $\pi^{ \pm} p$ interactions by

$N^{N e o n l y}\left(N^{\text {ohower }}, \ldots\right)=N^{\text {all }}\left(N^{\text {ohower }}, \ldots\right)\left(1-f_{H / N e} \cdot f_{H}\left(N^{\text {ehower }}\right) \cdot f_{\text {oiop }}\right)$

where

$$
\begin{aligned}
f_{H / N e} & =0.052 \\
f_{H}\left(N^{\text {ehower }}\right) & \text { is given by "fraction of events" } \\
f_{\text {viop }} & =\left(\begin{array}{c}
2 / 3 \\
1 / 3 \\
0
\end{array}\right) \text { for }\left(\begin{array}{c}
0 \\
1 \\
\geq 2
\end{array}\right) \text { visible p. }
\end{aligned}
$$

To correct for coherent scattering, the following values for the coherent cross section $\left(\sigma^{c o h}\right)$ in $\pi \mathrm{C}_{12}$ at $40 \mathrm{GeV} / \mathrm{c}$ are used $(0)$ :

\begin{tabular}{cc}
$\substack{\text { final } \\
\text { state }}$ & $\sigma^{c o h}$ \\
\hline$\pi \pi^{0} \pi^{0}$ & $(2.3 \mathrm{mb})$ \\
$\pi \pi^{+} \pi^{-}$ & $3.5 \mathrm{mb}$ \\
$\pi+2\left(\pi^{+} \pi^{-}\right)$ & $0.38 \mathrm{mb}$.
\end{tabular}

(The $\pi \pi^{0} \pi^{0}$ value is not a measured value but derived by isospin weighting ${ }^{[7]}$ from the $\pi \pi^{+} \pi^{-}$value.)

These values are scaled to the corresponding $\pi^{ \pm} \mathrm{Ne}$ values by using ${ }^{[8]}$

$$
\sigma^{o o h}(\pi \mathrm{Ne})=\left(\frac{A_{N_{e}}}{A_{C}}\right)^{2 / s} \sigma^{c o h}(\pi \mathrm{C})
$$

and the known energy dependence ${ }^{|8|}$ of $\sigma^{\text {coh }}$ ( $\pi$ nucleus). The resulting $\pi$ Ne expected fractions of coherent events $f_{\text {coh }}$ are:

\begin{tabular}{lcc} 
& $30 \mathrm{GeV} / \mathrm{c}$ & $64 \mathrm{GeV} / \mathrm{c}$ \\
\hline 1 prong: & 0.008 & 0.011 \\
3 prong : & 0.016 & 0.021 \\
5 prong : & 0.0014 & 0.0024
\end{tabular}


Of course, a $\pi^{ \pm} \mathrm{Ne}$ coherent event must have no identified protons, ie $N^{p}=0$.

Given these results, the WA51 distributions are corrected according to

$$
\begin{aligned}
N^{\text {non coh }}\left(N^{\text {ehower }},\right. & \left.N^{p}=0, \ldots\right)= \\
& N^{N e \text { only }}\left(N^{\text {ehower }}, N^{p}=0, \ldots\right)\left(1-f_{\text {coh }}\left(N^{\text {ehower }}\right)\right)
\end{aligned}
$$

for $N^{\text {ohower }} \in(1,3,5)$ and $N^{( \pm)} \in(1,2,3)$, respectively, for $\pi^{( \pm)}$incident.

Finally, because of the low scanning efficiency for 0 and 1 showertrack events in WA51, the cut $N^{\text {ehow }} \geq 2$ is required for all analyzed events. A corresponding cut ( $N^{\text {ohower }} \geq 2$ in the hadronic system) has been applied in the ${ }^{(} \bar{\nu}^{\prime} \mathrm{Ne}$ data for identical reasons. The net results of all the corrections discussed in this section are shown in table 4.2.1.

\section{4.8. Measured events.}

From the overall WA51 scan sample a representative sample was chosen for measurement. Measurements were done by the Seattle and Warsaw groups only. The goal of these measurements was to provide a representative sample with the incoming beam and all outgoing charged tracks measured for both $\pi^{+}$and $\pi^{-}$incident, so that (1) the inclusive distributions of the nonidentified protons, or $p_{\text {fast }}$ tracks, could be studied as per the analysis in section 7.5; and (2) to enable comparison with ${ }^{(} \bar{\nu}^{\prime} \mathrm{Ne}$ data under equivalent circumstances. Because of the greater complexity and general "messiness" of the $84 \mathrm{GeV} / \mathrm{c}$ events, only $\pi^{ \pm} \mathrm{Ne} 30 \mathrm{GeV} / \mathrm{c}$ data was measured.

\section{TABLE 4.2.1.}

\begin{tabular}{|c|c|c|c|c|c|}
\hline Bean & Totaㅛ & $\begin{array}{l}\text { Multiplicity } \\
\text { Cut. }\end{array}$ & $\begin{array}{c}\text { Coherent } \\
\text { Cus }\end{array}$ & $\begin{array}{l}\mathrm{H}_{2} \\
\mathrm{Cut}\end{array}$ & $\begin{array}{c}\text { Total } \\
\text { corrected }\end{array}$ \\
\hline$n^{+} 30 \mathrm{GeV} / \mathrm{c}$ & 3858 & 273 & 60 & 180 & 3345 \\
\hline$\pi^{\sim} 30 \mathrm{GeV} / \mathrm{c}$ & 5729 & 283 & 90 & 259 & 4997 \\
\hline$n^{+} 64 \mathrm{GeV} / \mathrm{c}$ & 1516 & 64 & 34 & 63 & 1355 \\
\hline $64 \mathrm{ccV} / \mathrm{c}$ & 1450 & 65 & 32 & 70 & 1283 \\
\hline
\end{tabular}

Analyzed events in WA51, showing corrections. 
Events measured by the Warsaw group were processed through an offline system, where the event is measured and stored on magnetic tape, and later reconstructed by a geometry program to teat the quality of the measurement. This unfortunately leads to a series of remeasures of remeasures, where sheer bookkeeping problems nearly guarantee that some events will have failing tracks.

Seattle measurements were done on a film-plane measuring device which had a setting error of about $3 \mu$, known as the BRIDE, for BRIght DEvice (ie, it had an onboard microprocessor). The BRIDE was interfaced to a PDP10 computer, on which an overall supervisory program for the BRIDE measurements ran. The program was named ONLINE, since this was a full online measurement system. By "full online", I mean that the final results for the measurement were generated at measurement time, so that the event reconstruction is completely finished at the end of the measurement.

The ONLINE program demanded that all charged tracks in the event be measured before the event could be declared finished, and handled all bookkeeping by generating a catalogued output for each event. The raw data was also recorded in a separate log fle in case any later revisions or catastrophic losses forced reprocessing. For geometry reconstruction, ONLINE used the HYDRA bubble-chamber geometry program developed at CERN, with suitable modifications for the Seattle measuring environment. The order of measurement was (1) fiducials, (2) all vertices, and (3) all tracks, with reconstruction done after each vertex and each track. All track and vertex matching was by hand (ie the BRIDE operator), which is feasible for the $\pi^{ \pm} \mathrm{Ne} 30 \mathrm{GeV} / \mathrm{c}$ data but difficult for more complicated events.

The reconstruction requirements used by ONLINE were that a fitted vertex have at least a $3 \%$ probability of being a single point fit, and that a fitted track have $\delta p / p \leq 0.2$ for a $\pi$ or a $p$ mass fit, with a minimum of 3 views participating in the fit. If a track measurement still failed this criteria after 3 measurements, the BRIDE operator was allowed to fag the track and proceed; the best of the 3 measurements was kept for the output structure.

The total number of measured events in WA51 are: $\pi^{+} \mathrm{Ne} 30 \mathrm{GeV} / \mathrm{c}$, 595 events; $\pi^{-} \mathrm{Ne} 30 \mathrm{GeV} / \mathrm{c}, 583$ events.

Since the multiplicity distribution of the events in the measurement sample differs from that of the total scan sample, measured events in $\pi^{+} \mathrm{Ne}$ and $\pi^{-} \mathrm{Ne}$ are weighted to meet two requirements. First, that the weighted measurement shower track multiplicity distribution be the same as that for the full corrected scan sample with the same beam. Second, that the sum of all weighted events be the same as the original number of events in the measurement sample (it is convenient to retain this normalization because distributions then still give a "feel" of the absolute number of events and/or tracks involved). That is, the weight of an event with $n$ shower tracks is

$$
w(n)=\frac{N^{\bullet}(n)}{N^{m}(n)} \frac{\sum_{n} N^{m}(n)}{\sum_{n} N^{\bullet}(n)},
$$

where $m$ labels the measurement sample, olabels the scan sample, and $N(n)$ is the number of events with $n$ shower tracks. 


\section{Notes for Chapter 4}

1. W.M. Yeager et al., Phys. Rev. D16, 1294 (1977)

2. Experiment WA52 was carried out by the Aachen-Bonn-CERNLondon-Oxford-Saclay Collaboration. See P. Fritze et al., Phys. Lett. $96 B, 427(1980)$

3. A.K. Wroblewski, "Topologic Cross Sections in $\pi p$ Interactions" University of Washington, Visual Techniques Laboratory, Physics Note PHY-90 (1982)

4. J.C. Allaby et al., Sov. J. Nucl. Phys. 12, 295 (1971); Yu. P. Gorin et al., Sov. J. Nucl. Phys. 18, 173 (1974)

5. A.K. Wroblewski, private communication

6. E.V. Anzon et al., Phys. Lett. 31B, 241 (1970); also B. Yuldashev, private communication

7. F. Cerulus, Nuov. Cim. 19, 528 (1961)

8. V.G. Grishin, B.S. Yuldashev, and G. Jancso, Sov. J. Nucl. Phys. 14, 712 (1872)

\section{CHAPTER 5}

\section{ANALYSIS TECHNIQUES}

\section{5.1. Comparion of ${ }^{(-\bar{\nu})}$ and $\pi^{ \pm}$lneldent.}

When comparing $(\bar{\nu}) \mathrm{Ne}$ snd $\pi^{ \pm} \mathrm{Ne}$ systems, the two systems should be at the same hadronic CM energy. Since, as discussed earlier, the E546 ${ }^{\prime}(\bar{\nu}) \mathrm{Ne}$ data cover a wide range of hadronic CM energies, this implies choosing a band in the $(-\bar{\nu}) \mathrm{Ne}$ data to correspond to the $30 \mathrm{GeV} / \mathrm{c}$ (incident momentum) $x^{ \pm} \mathrm{Ne}$ data and $64 \mathrm{GeV} / \mathrm{c} \boldsymbol{x}^{ \pm} \mathrm{Ne}$ data. Due to different historical developement, the square of the hadronic CM energy is called $W^{2}$ in neutrino interactions, and $s$ in hadronic interactions. Clearly we should have $W^{2}=\boldsymbol{s}$.

The general technique for choosing a band width in $W^{2}$ is to compromise between taking the narrowest possible band width while leaving adequate statistics for the task at hand, choosing a band in $W^{2}$ of the 
form $\left\langle W^{2}\right\rangle-\Delta W^{2} / 2 \leq W^{2} \leq\left\langle W^{2}\right\rangle+\Delta W^{2} / 2$, where $\Delta W^{2}$ is the width of the band. Since this dissertation is primarily concerned with nuclear effects on the scattering system, and since the primary measure of level of nuclear effects is the multiplicity $N$, the band for comparison between $\pi^{ \pm} \mathrm{Ne}$ and $(\bar{\nu}) \mathrm{Ne}$ is chosen for constant width in $(N)$, instead of constant width in $\boldsymbol{W}^{2}$.

As is discussed in section 6.6, the multiplicity in both neutrino and hadron scattering can be fit by the form $(N)=a+b \cdot \ln \left(W^{2}\right)$ (in hadron scattering there is sufficient range and sufficient accuracy that usually a "scaling violation" term $c \cdot \ln ^{2}\left(W^{2}\right)$ is also included in the fit). Thus to have a constant width in $N$ implies choosing a constant width in $\ln \left(W^{2}\right)$ instead of $W^{2}$.

The constraints chosen to determine the $W^{2}$ band used in comparison were: (1) $\left\langle W^{2}\right\rangle=s$, and (2) $\Delta N=1$, that is, the band is 1 unit wide in in multiplicity. Constraint number (2) uses only the slope parameter $b$ in the multiplicity 6t, which is the more stable of the two parameters. The $\mathrm{CM}$ energy, or $\sqrt{s}$, for $30 \mathrm{GeV} / \mathrm{c} \pi^{ \pm}$is $7.56 \mathrm{GeV}$, and $\sqrt{s}$ for $64 \mathrm{GeV} / \mathrm{c}$ $x^{ \pm}$is 11.08. Using these values and the slope parameters given in table 6.6.1, the $W^{2}$ bands are determined to be:

$$
\begin{array}{ll}
30 \mathrm{GeV} / \mathrm{c}: & 6.25 \leq W \leq 8.88 \\
64 \mathrm{GeV} / \mathrm{c}: & 9.16 \leq W \leq 13.01
\end{array}
$$

The $W^{2}$ distribution for the $\mathbf{E 5 4 6}$ data over these ranges is essentially flat, implying that no weighting corrections are necessary for data taken in these bands.

\subsection{The NUCTST algorlthm.}

The NUCTST algorithm is used extensively to obtain the results presented in this dissertation. This algorithm places $\nu \mathrm{Ne}$ and $\mathrm{vNe}$ events into three categories: (a) nuclear events, or events that show evidence of having nuclear reinteractions; (b) nonnuclear events, or events that are compatible with no nuclear reinteractions, $i e^{(} \bar{\nu}^{\prime} N$; and (c) undecided events, or events where some aspect of the event prevents a clear choice of nuclear or nonnuclear. The NUCTST algorithm also has an option designed to improve statistics, where undecided events are placed into either the nuclear or nonnuclear category with a weight corresponding to a pseudo-probability that such an assignment is correct.

This section discusses the implementation of the NUCTST algorithm.

If any of four criteria are met and if the event is not undecided, the event is classified as nuclear. These four criteria are: (1) the event contains more than one visible proton, subject to a momentum constraint on the protons (see below); (2) the event has any "backwards" protons in the lab frame; (3) any non-proton non-gamma track in the event has $x_{F}$ (Feynman $x$ ) less than or equal to -1.0 ; and (4) the net charge of the event is inconsistent with the type of incident $\boldsymbol{W}$ interacting with $\boldsymbol{n}$ or $\boldsymbol{p}$.

Criterion ( 1 ) is used because clesrly there can be a maximum of one visible proton in the event if the interaction is on a single nucleon, and other protons must come from the Ne nucleus (A similar decision criterion could be implemented for neutrons, but neutron identification in this experiment is essentially nonexistant. Even in events with a recorded neutral star - a "mishmash" of tracks from a neutral vertex, presumably due to $n+N e \rightarrow$ ? - there is no unambiguous way to associate the $n$ 
with the event.).

However, even in cases where the interaction is on a single nucleon in $\mathrm{Ne}$, and this aucleon then leaves the Ne nucleus without further interaction, the absence of the nucleon may leave the nucleus in an excited state, from which it then decays to generste visible protons. Since the fermi momentum for $\mathrm{Ne}$ is $k_{F}=1.35 \mathrm{fm}^{-1} \approx 270 \mathrm{MeV} / \mathrm{c}$, this establishes an absolute upper limit for visible protons from such a case. However most protons in this case will be far below $\boldsymbol{k}_{\boldsymbol{F}}$, so the limit taken is $200 \mathrm{MeV} / \mathrm{c}$. Thus criterion (1) applies only to visible protons with $p \geq 200 \mathrm{MeV} / \mathrm{c}$. However backwards protons are taken as strong evidence of direct nuclear involvement, leading to criterion (2).

$x_{F}$ has the bounds $\left|x_{F}\right| \leq 1.0$, where we evaluate $x_{F}$ in the CM and assume the initial interaction is $W^{ \pm}+n(p) \rightarrow$ (anything). This leads to criterion (3), as only a nuclear effect can take $x_{F}$ out of this range. This criterion for $x_{F}$ is used as opposed to taking tracks with backwards momenta in the lab because resonance decays to particles with much less mass than the parent resonance (for exsmple, $p^{0} \rightarrow \pi^{+} \pi^{-}$) can throw a decay product into the backwards hemisphere in the lab frame, using the available mass energy.

Clearly charge balance must be consistent with the interaction type unless additional charge comes from the nucleus. However, in checking charge balance, we are assuming that the sign of the charge of all charged tracks in the event is well known. For too high a relative error in the momentum $(\delta p / p)$, or for a failing charged track, this is not the case.

From the geometry, we have

$$
\frac{\delta p}{p}=\frac{\delta k}{k} \equiv \frac{1}{N_{o}}
$$

where $k$ is the track curvature, and so the variance of the curvature $(\sigma)$ is $\sigma=\delta k$. From figure 5.2.1, we see that we must go $N_{\sigma} \cdot \sigma$ to reverse the sign of $k$. Thus the probability the sign of $k$ is wrong is

$$
P_{\bullet}\left(N_{\sigma}\right)=\frac{1}{\sqrt{2 \pi}} \int_{N_{\sigma}}^{\infty} e^{-x^{2} / 2} d x
$$

ie the area under the gaussian beyond $k=0$ away from $k$ (the cross-hatch area in figure 5.2.1). $P_{0}$ is evaluated by noting that this is just $1 / 2$ the confidence level (ie including the dotted area in figure 5.2.1 also).

The bound chosen for having a "well known" sign of the charge is $2 \sigma$, or $95 \%$, which by the above calculation corresponds to $\delta p / p \leq 0.6$. If an event contains a track that exceeds this limit, it is placed in the undecided category; otherwise, charge balance is applied.

Applying the NUCTST algorithm to the $\mathrm{E} 546 \nu \mathrm{Ne}$ dats, for example, $33 \%$ of all events fell in the nuclear category, $54 \%$ fell in the nonnuclear category, and the remaining $13 \%$ were undecided. Of those events assigned to the nuclear category, $43 \%$ were assigned because of a backwards proton, $15 \%$ for more than 1 visible proton, $5 \%$ for having $x_{F} \leq-1$, and $37 \%$ for failing charge balance (Some care is necessary because these numbers are contingent on the order of evaluation of the criteria. For the numbers cited, the order of evaluation was the same as the order in which the percentages are given.). To check that no systematics were introduced by the proton cuts (through choice of the visible proton sample, say), $\left\langle W^{2}\right\rangle$ and $\left\langle Q^{2}\right\rangle$ were plotted as functions of the number of visible protons $\left(N_{p}\right)$ in the nuclear sample. No variation is seen. 


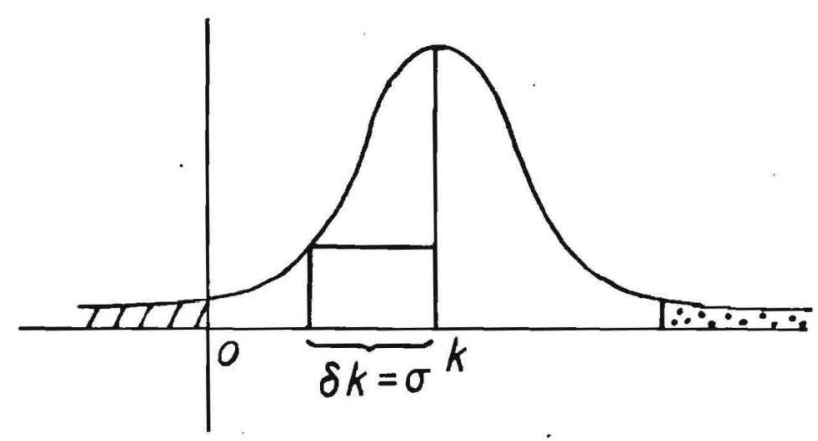

FIGURE 6.8.1.

Computing the probability the sign of the charge is wrong from a Gaussian error distribution in curvature. (See text)
In the option where the undecided events are entered into a nuclear or nonnuclear category with a weight corresponding to a psuedo-probability, the weight is assigned as follows. All charge tracks failing the $\delta p / p$ requirement are ordered according to the probability that the sign of their charge is wrong, ie according to $P_{\text {. }}$. Let $n$ be the number of tracks needed to bring the event into charge balance correspondence with the category to be assigned (either nuclear or nonnuclear). If $n$ is less than or equal to the number of indeterminate tracks in the event, we take the $n$ tracks with highest $P_{0}$, and the weight is then

$$
w=1-\prod_{i}^{n}\left(1-P_{0}(i)\right)
$$

If $n$ is greater than the number of indeterminate tracks in the event, the weight is 1.0 or 0 , depending on the calegory.

Clearly this weight has the correct behavior. If a large number of tracks must change sign to meet the event criteria, the weight is small, even if the probability for all tracks is large. The weight can only be large if a single track is considered, and if the probability for that track's sign to be the correct sign for the category considered is also large. A study of distributions with weighted events compared to distributions without weighted events found no significant differences, other than some improvement in statistics.

The goal of the NUCTST algorithm is not to assign all individual events correctly to the three categories, but to divide the data into samples of events whose overall comparison gives insight into nuclear vs. nonnuclear processes. Some contamination of events from the wrong category will be present in other categories, but we are trying to determine only in 
what manner the quantities under investigation change if we enrich the nuclear content of the sample, or enrich the nonnuclear content of the sample. For this purpose the algorithm is adequate.

Finally, a matter of notation. In reference to the manner in which the NUCTST algorithm is actually coded, in figures and tables the nuclear category is often referred to as "NUCTST $=+Z$, the nonnuclear category as "NUCTST $=-Z$, and the entire data sample as "NUCTST $=\sigma$. These categories are not purely nuclear or nonnuclear, but only enriched samples as decided by the NUCTST algorithm.

\section{5.8. $W^{2}$ welghting.}

When comparing $\nu \mathrm{Ne}$ and $D \mathrm{Ne}$ data in E546, if the range of comparison is over a broad range in $W^{2}$ (for example, the entire data sample), then the $W^{2}$ distributions of the two samples can be significantly different. Since nuclear effects and indeed properties of the final state hadronic system in general are a strong function of $W^{2}$, spurious differences may be introduced into the comparison because of the different $W^{2}$ distributions. (As discussed in section 6.6, other leptonic variables have little effect on the final state hadronic system for the quantities examined in this dissertation.)

To overcome this problem, a system of $W^{2}$ weighting was used to guarantee that two dats samples with a large width in $W^{2}$ would have the same $W^{2}$ distribution. This section describes the system of $W^{2}$ weighting used. (It should be stressed that such weighting is necessary only for very large $\Delta W^{2}$ samples, which in practice nearly always means for com- parisons of the entire data sample. Whenever $W^{2}$ weighting has been used, notation to that effect is made.)

Assume that the two samples are divided into $j$ bins each, all of constant width $\Delta W$. Then each one of these $\Delta W$ bins will contain $N_{i}$ total events and $n_{i}$ total tracks ( ${ }^{i}{ }^{*}$ is the bin label, and $i$ runs from 1 to $j$ ). Let the weight assigned to each bin be $w_{i}$. Then there are three obvious weighting conditions:

$$
\begin{aligned}
w_{i}^{a} N_{i}^{a} & =w_{i}^{b} N_{i}^{b} \quad(i=1 \ldots j) \\
\sum_{i} w_{i}^{a} N_{i}^{a} & =1 \\
\sum_{i}^{i} w_{i}^{b} N_{i}^{b} & =1
\end{aligned}
$$

In these conditions, $a$ labels sample no. 1 , and $b$ labels sample no. 2 (usually $\nu \mathrm{Ne}$ and $D \mathrm{Ne}$ ). Condition 5.3 .1 forces the $W^{2}$ distributions to be equivalent for the two samples. Conditions 5.3.2 and 5.3.3 express the fact that the $w_{i}$ are normalized weights, that is $\sum w_{i}^{a} n_{i}^{0}=\langle n\rangle_{a^{\prime}}$, where the sum runs over all tracks. The weights are taken to be normalized weights because the weighting is done for inclusive distributions, and normalized weights are appropriate for distributions of the form $1 / \sigma d \sigma / d \theta$, where $\theta$ is any kinematic variable.

There are $2 j$ total weights and $j+2$ constraints, so for any practicle $\Delta W$ this constitutes an underconstrained system. One can invert $2 j-(j+$ 2) constraints to find a weight set, but this carries with it the possibility of biasing the sample: for example, one could weight a bin so that it had a negative number of events in it. Also ideally one would like to have the $w_{i}$ independent of the $n_{i}$

The solution adopted here is to preserve the total number of events in 
each combined bin $N_{i}=N_{i}^{a}+N_{i}^{b}$ by weighting the events so that there is an equal number of events in a given $i$ bin for both $a$ and $b$ samples. Then in the total sample $a+b$ exactly half the total events $N=\sum N_{i}$ are in the $a$ sample, and the other half are in the $b$ sample. Then the normalized weights are found by dividing out $N / 2$ from each sample. The final weights are

$$
w_{i}^{a}=\frac{N_{i}^{a}+N_{i}^{b}}{N_{i}^{a} \cdot \sum_{i}\left(N_{i}^{a}+N_{i}^{b}\right)},
$$

and $w_{i}^{b}$ is given by interchanging $a$ and $b$.

For the actual implementation of $W^{2}$ weighting, the $W$-width $\Delta W$ was $1 \mathrm{GeV}$. Since the weighting technique requires prior knowledge of the $W$ distribution of the events (which can change as cuts, etc., change), for calculations involving weighted data the $W$ distribution of the events selected was accumulated. After all events had been accumulated, the final $W$ distribution ras required to agree with the input $W$ distribution used to calculate weights. If the distributions did not agree, the analysis was discarded, and a new $W$ distribution was recorded for use in subsequent analysis.

\section{CHAPTER *}

\section{MULTIPLICITY RESULTS}

\section{6.1. Chapter Overvlew.}

This chapter deals with multiplicity-related results and their comparison between the ${ }^{(-)} \mathrm{Ne}$ system and the $\pi^{ \pm} \mathrm{Ne}$ system at equiralent hadronic CM energies. Multiplicities are strongly affected by nuclear reinteractions and provide a key measure of the importance, effects, and mechanism of such reinteractions. In view of the important role multiplicities play in analyzing nuclear reinteractions, the WA51 scan phase was specifically designed to take grest care with multiplicity features in $\pi^{ \pm} \mathrm{Ne}$. The comparison of these multiplicity results for $\pi^{ \pm} \mathrm{Ne}$ with those for $\left(\bar{\nu}^{\prime}\right) \mathrm{Ne}$ probes differences between hadronic and weak interaction systems shortly after their formation.

Section 6.2 studies various features of shower (ie minimum ionising) 
track distributions in the two systems. A linear relationship between the dispersion in $N_{p r}^{-}, D_{p r}^{-}$(see section 6.2 for the definition of $N_{p r}^{-}$), and $\left\langle N_{p r}^{-}\right\rangle$is found, but the $\left(\bar{\nu}^{\prime}\right)$ Ne slope and intercept differ from the universal hadron-hadron values. $C_{\mathrm{pr}}^{k-}$ moments are studied, and the ratio of average $N$ with a nuclear target to a nucleon target $\left(R_{A}\right)$ is presented for $\pi^{ \pm} \mathrm{Ne}$ and $(-\bar{\nu}) \mathrm{Ne}$. The $R_{A}$ dependence on the hadronic CM energy is found, and $R_{A}$ for $(\bar{\nu}) \mathrm{Ne}$ is considerably below $R_{A}$ for $\pi^{ \pm} \mathrm{Ne}$ at equivalent energies.

Section 6.3 presents features of identified and non-identified proton distributions (non-identified protons have sufficient momentum to be minimum ionizing, and are called $p_{\text {faot }}$ tracks). The section includes a derivation of the Andersson model ${ }^{[1]}$ of proton production and comparison to the data. We find about 0.6 feot tracks per event, with slightly more in $\left({ }^{\prime}\right)$ Ne than $\pi^{ \pm} \mathrm{Ne}$. There is no evidence of correlation between identifled proton and $p_{\text {faot }}$ production, but this may be a saturation effect in $\mathrm{Ne}$, with larger nuclei possibly showing a correlation.

Section 6.4 desls with correlations between nuclear reinteractions and the number of identified protons, $N_{p}$. Clearly $N_{p}$ provides a measure of the average number of nuclear reinteractions, $\langle\nu\rangle . N_{p r}^{-}$and $D_{p r}^{-}$, rise linearly with $N_{p}$. The slope of $D_{p r}^{-}\left(N_{p}\right)$ is compared for $(-) \mathrm{Ne}$ and $\pi^{ \pm} \mathrm{Ne}$, and the relation between $\langle\nu\rangle$ and $N_{p}$ is plotted for both systems. This relation is, as we might expect, evidently a universal function. $C_{p r}^{k-}$ moments are presented as a function of $N_{p}$ (or equivalently $\langle\nu\rangle$ ).

Section 6.5 studies KNO scaling ${ }^{[2]}$ for many different aspects of $\pi^{ \pm} \mathrm{Ne}$ and ${ }^{(} \bar{\nu}^{-} \mathrm{Ne}$ systems, and discusses some of the caveats involved with the ides of KNO scaling. In particular, there is strong evidence that KNO scaling has its origin in statistical properties of production systems, as opposed to dynamical properties. KNO scaling is found to hold at least as well when comparing events with no nuclear reinteractions to events with nuclear reinteractions as in other types of comparisons.

Section 6.6 looks at the relationship between multiplicity features and primary event variables in $\left({ }^{-}\right) \mathrm{Ne}$, such as $Q^{2}, W^{2}, \nu, x_{B J}$, etc. The only important variable is found to be $W^{2}$, as opposed to some prior papers ${ }^{|3|}$. The behavior of many types of multiplicity moments, such as the skewness and the integral of the two-particle correlation function, are presented as a function of $W^{2}$. Several ty pes of multiplicity distribution are shown to obey the scaling law $\langle N\rangle=a+b \cdot \ln \left(W^{2}\right)$ well, and the parameters $a$ and $b$ are compared for many types of scattering systems. A detailed search for any $Q^{2}$ dependence shows no dependence whatsoever.

\section{6.2. Shower track multiplicity distributions.}

This section presents various features of the multiplicity distributions measured in experiments E546 and WA51. As discussed in Chapter 2, the increase in multiplicity for interactions on nuclei as opposed to interactions on nucleons is a direct measure of reinteractions in the nucleus. Thus studying multiplicity distributions can shed light on the mechanism of reinteraction. Comparing distributions between $\pi^{ \pm} \mathrm{Ne}$ and $\left(\bar{\nu}^{\prime}\right) \mathrm{Ne}$ provides yet another probe of nuclear reinteractions, since the probability of reinteraction in $\pi^{ \pm} \mathrm{Ne}$ is roughly $1 \frac{1}{8}$ to $1 \frac{1}{2}$ that of reinteraction in $(\bar{\nu}) \mathrm{Ne}$.

Many of the following multiplicity results are presented in the variable $N_{p r}^{-}$. This eliminates any contamination by non-identified protons and compensates for the different initial charge present when comparing 
incident-particle and incident-antiparticle. $\left\langle N_{p r}^{-}\right\rangle$ів defined as

$$
\left\langle N_{\mathrm{pr}}^{-}\right\rangle=\sum_{N^{-}-1} N^{-} \sigma_{N^{-}} / \sum_{N^{-}-1} \sigma_{N^{-}}
$$

for incident $x^{+}(\nu)$ and as

$$
\left\langle N_{\mathrm{pr}}^{-}\right\rangle=\sum_{N^{-}-2}\left(N^{-}-1\right) \sigma_{N^{-}} / \sum_{N^{-}-2} \sigma_{N^{-}}
$$

for incident $\pi^{-}(D)$. Taking the summation over $N^{-} \geq 2$ for incident negative charge and over $N^{-} \geq 1$ for incident positive charge eliminates contamination from quasi-elastic interactions.

Similarly $D_{\mathrm{pr}}^{-}$is defined as

$$
D_{p r}^{-}=\sqrt{\left\langle\left(N_{p r}^{-}\right)^{2}\right\rangle-\left\langle N_{p r}^{-}\right\rangle^{2}} .
$$

Other moments used in this chapter are given in section 1.2.

Figure 6.2.1 shows the dispersion $D_{p r}^{-}$plotted against the average multiplicity $\left\langle N_{p r}^{-}\right\rangle$. It has long been recognized that the dispersion is a universal linear function of the average multiplicity for all energies for a given interaction type ${ }^{|4|}$. (However, different slopes and intercepts may be needed for different types of incident particle. For example, different straight lines may be needed for $e^{+} e^{-}$compared to hadron-badron scattering.)

This result is expected in the limit where KNO scaling holds. If one plots $P(n)$ versus $n$, where $P(n)$ is the probability of observing $n$ tracks, then the normalization condition $\int P(n) d n=1$ fixes the area under the curve to 1 . Rescaling the $n$ axis by $(n)^{-1}$ and the $P(n)$ axis by $(n)$ preserves the normalization. Then the half-width of this curve $\sim D /(n)$. If all the

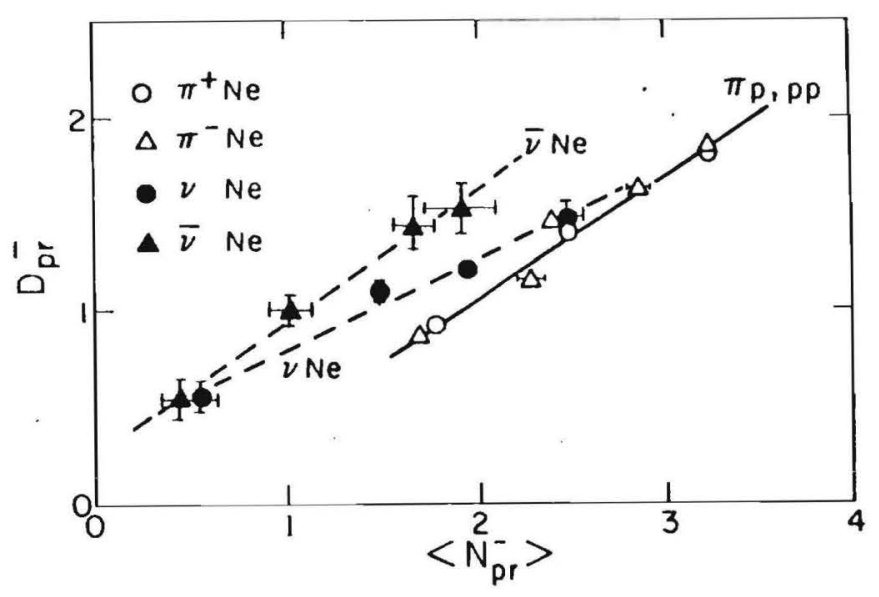

FIGURE 6.8.1

The dispersion $D_{\mathrm{rr}}^{-}$versus average multiplicity $\left\langle N_{\mathrm{pr}}^{-}\right\rangle$. The $*$ Ne data are at $10.5^{\mid 10]}, 25^{\mid 11]}, 30,50^{[11]}$, and $64 \mathrm{GeV} / \mathrm{c}$. The solid line represents a parameterization of $x p$ and $p p$ data ${ }^{[8]}$. The $\nu \mathrm{Ne}$ and $\nu \mathrm{Ne}$ data are from E546, extrapolated to zero bin width. The 30 and $64 \mathrm{GeV} / \mathrm{c} \approx \mathrm{Ne}$ data are from WA51. 
scaled curves are to be identical, as KNO asserts, they must have the same width, so $D /\langle n\rangle=$ Const. Thus deviations from the linear relationship measure deviations from KNO scaling.

Figure 6.2.1 shows that $\pi \mathrm{Ne}, \pi p$, and $p p$ data all fall on the same straight line to within a few percent. $\nu \mathrm{Ne}$ and $\nu \mathrm{Ne}$ fall on straight lines on the plot, but these lines are different from the hadron-Ne line, and also different from each other, having different slopes. The $\bar{\nu}$ Ne line has the same slope as the hadron-Ne line within errors; the $\nu \mathrm{Ne}$ line has a significantly smaller slope. This result is similar to the result obtained in $(\bar{\nu})^{|5|}$.

Average multiplicities and dispersions for $\pi^{ \pm} \mathrm{Ne}$ and ${ }^{(} \bar{\nu}^{\prime} \mathrm{Ne}$ are shown in table 6.2.1. Averages for $N^{+}$(positive minimum-ionizing tracks with identifled protons excluded) and $N^{-}$(negative minimum-ionizing tracks) are given, as well as the average, dispersion, and their ratio, in $N_{p r}^{-}$. Values for $p \mathrm{Ne}$ at $28 \mathrm{GeV} / \mathrm{c}^{[0]}$ and $300 \mathrm{GeV} / \mathrm{c}^{[7]}$ are included for comparison. $\left(N^{+}\right)$and $\left\langle N^{-}\right\rangle$tend to be about $0.4-0.5$ lower for $\nu$ Ne than for $\pi$ Ne. $\left\langle N_{p r}^{-}\right\rangle$tends to be about 0.6 lower for $\nu$ Ne while $D_{p r}^{-}$is much closer to the $\pi$ Ne values.

In addition to the dispersion, in the limit of exact KNO scaling the normalized higher-order moments, defined as

$$
C_{p r}^{k-}=\frac{\left\langle\left(N_{p r}^{-}\right)^{k}\right\rangle}{\left\langle N_{\overline{p r}}\right\rangle^{k}},
$$

are identical for all energies for the same scattering system. (The $C_{\mathrm{pr}}^{\mathbf{k}}$ are assumed to determine the KNO scaling function $\psi(z)$ via an integral expansion $\int \psi(z) z^{k} d z=C_{p r}^{k-}$. For a complete discussion of KNO scaling,
TABLE 6.2.1.

Average multiplicities and dispersions.

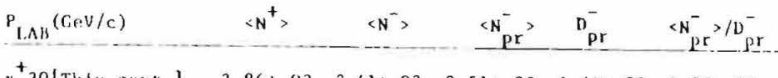

$$
\begin{aligned}
& \begin{array}{llllll}
30 \text { This expt.] } & 3.861 .03 & 2.41 \pm .03 & 2.51 \pm .02 & 1.40 ! .02 & 1.80 * .03
\end{array} \\
& \begin{array}{llllllll}
030 \quad \text { " } & 3.68 \pm .04 & 2.06 \pm .03 & 2.03 \div .04 & 1.31 \pm .04 & 1.55 \div .06
\end{array} \\
& \begin{array}{lllllll}
\pi & -30 \quad 3.09 \pm .03 & 3.19 \pm .02 & 2.43 \pm .02 & 1.44 \pm .02 & 1.68 \pm .03
\end{array} \\
& \begin{array}{lllllll}
330 \quad \text { " } & 2.61 \div .08 & 2.70 \div .08 & 1.86 \pm: 11 & 1.47 \div .09 & 1.26 ! .11
\end{array} \\
& \begin{array}{llllllll}
{ }_{n}^{+} 64 \quad " \quad 4.55 \pm .06 & 3.17 \pm .05 & 3.24 \pm .05 & 1.87 \pm .04 & 1.78 \pm .05
\end{array} \\
& \begin{array}{llllllll}
064 & \| & 4.12 \div .04 & 2.52+.05 & 2.63 \pm .05 & 1.54 \pm .04 & 1.71 \pm .05
\end{array} \\
& \begin{array}{lllllll}
{ }^{-} 64 & \text { " } & 3.87 \pm .06 & 4.06 \pm .05 & 3.26 \pm .05 & 1.86 \pm .04 & 1.75 \pm .05
\end{array} \\
& \begin{array}{llllllll}
\bar{v} 64 \quad " & 3.32 ! .11 & 3.38 \pm .10 & 2.42 \pm .14 & 1.61 ! .14 & 1.50 \pm .16
\end{array} \\
& \begin{array}{llllllll}
\pi^{+} 10.5 & {[10]} & 2.86 \pm .04 & 1.37 \pm .02 & 1.77 \pm .07 & 0.92 \pm .07 & 1.92 \pm .02
\end{array} \\
& \begin{array}{llllllll}
{ }^{-} 10.5 & {[10]} & 1.83 \div .02 & 2.08 \pm .02 & 1.69 \pm .07 & 0.86 \pm .07 & 1.96 \pm .02
\end{array} \\
& \begin{array}{lllllll}
\pi & -25 & {[11]} & 2.60 \pm .07 & 2.29 \pm .07 & 1.16 \div .05 & 1.97 \div .07
\end{array} \\
& \begin{array}{llllll}
{ }^{-} 50 & 111] & 3.41 \pm .07 & 2.85 \div .06 & 1.63 \div .05 & 1.75 ! .05
\end{array} \\
& \begin{array}{lllll}
p 28 & {[6]} & 2.33 \pm .08 & 1.31 \div .06 & 1.77 \pm .07
\end{array} \\
& \begin{array}{llllll}
\text { p } 300 \quad[7] & 5.40 \div .04 & 3.554 .04 & 1.52 \pm .01
\end{array}
\end{aligned}
$$




\section{TABLE 6.8.8.}

$C_{p r}^{k-}$ moments for $N_{p r}^{-}$distributions in $\pi^{ \pm} \mathrm{Ne}$ and $\left(\bar{\nu}^{\prime}\right) \mathrm{Ne}$. The ${ }^{(} \bar{\nu}^{\prime} \mathrm{Ne}$ ralues are extrapolated to zero bin width.

\begin{tabular}{|c|c|c|c|c|}
\hline \multicolumn{2}{|c|}{$P_{L A B}(G e V / c)$} & $\mathrm{c}_{2}^{-p r}$ & $\mathrm{c}_{3}^{-\mathrm{pr}}$ & $\mathrm{c}_{4}^{-\mathrm{pr}}$ \\
\hline \multicolumn{2}{|c|}{$n^{+} 30$ [This expt.] } & $1.31 \pm 0.01$ & $2.10 \pm 0.04$ & $3.92 \div 0.13$ \\
\hline$\checkmark 30$ & $"$ & $1.41 \div 0.02$ & $2: 54 \pm 0.11$ & $5.53 \div 0.50$ \\
\hline$\approx 30$ & $"$ & $1.35 \pm 0.01$ & $2.30 \pm 0.04$ & $4.61 \pm 0.13$ \\
\hline ¿ 30 & $"$ & $1.63=0.07$ & $3.41 \div 0.33$ & $8.46 \div 1.38$ \\
\hline$x^{+} 64$ & $"$ & $1.31 \pm 0.01$ & $2.12 \pm 0.06$ & $4.05=0.25$ \\
\hline .64 & $"$ & $1.34 \div 0.02$ & $2.23 \pm 0.08$ & $4.41 \div 0.29$ \\
\hline$n^{-64}$ & $"$ & $1.33 \div 0.01$ & $2.15 \pm 0.06$ & $4.00 \div 0.20$ \\
\hline $\bar{v}_{64}$ & $"$ & $1.44 \pm 0.06$ & $2.66 \pm 0.33$ & $5.95=1.34$ \\
\hline$n^{+} 10.5$ & {$[10]$} & $1.27 \div 0.01$ & $1.98 \pm 0.02$ & $3.63 \div 0.07$ \\
\hline${ }^{-} 10.5$ & {$[10\}$} & $1.26 \pm 0.01$ & $1.93 \div 0.02$ & $3.46 \div 0.07$ \\
\hline$m^{-} 25$ & {$[11]$} & $1.26: 0.02$ & $1.87 \pm 0.07$ & $3.14 \pm 0.21$ \\
\hline$\pi$ - 50 & [11] & $1.32=0.02$ & $2.12 \pm 0.07$ & $3.93 \div 0.24$ \\
\hline
\end{tabular}

see section 6.5. ). The $C_{p r}^{k-}$ moments are given in table 6.2.2. These moments for $\pi^{ \pm} \mathrm{Ne}$ are remarkably similar for different energies, but differ considerably from the ${ }^{(} \bar{\nu}^{\prime} \mathrm{Ne}$ moments. The $\left.{ }^{(}\right)^{\prime}$ moments also show considerable variation amongst themselves, with the $\bar{\nu}$ moments greater than the $\nu$ moments at equivalent energies.

The ratio of an average multiplicity in nuclear scattering to the same average multiplicity in nucleon scattering can only be sensitive to differences due to nuclear effects, provided there are no systematic differences in the two measurements. Thus the ratio

$$
R_{A}=\frac{(N)_{N e}}{\langle N\rangle_{p}}
$$

plays a key role in snaly zing nuclear effects.

Table 6.2.3 compares $R_{A}$ for $\pi^{ \pm} \mathrm{Ne}$ and $\left.{ }^{(} \bar{\nu}^{\prime}\right) \mathrm{Ne} . R_{A}$ (minus) is computed using $\left\langle N^{-}\right\rangle$and $R_{A}(a l l)$ is computed using $\left\langle N^{\text {ehower }}\right\rangle$. The values for $(N\rangle_{s p}$ are computed using an interpolation given in ${ }^{[8]}$. Two sets of values for $R_{A}\left(\bar{\nu}^{\prime} N e\right)$ are given; one set is calculated using BEBC fitg ${ }^{[b]}$ to $\bar{\nu}^{\prime} p$, the other is called $\tilde{R}_{A}^{(2)}$ and is calculated using the NUCTST algorithm. The assumption is that NUCTST $=-2$ events represent a good approximation $\operatorname{lo}\left(\bar{\nu}^{\prime} p\right.$ events, taken as a statistical sample. Using $\tilde{R}_{A}^{(2)}$ has the advantage of avoiding any systematic or normalization differences between BEBC and E548 (the BEBC data have been adjusted using results of a Monte Carlo based on a theoretical model, which may skew their results relative to $\mathrm{E} 546) . \tilde{R}_{A}^{(2)}$ is defined as

$$
\tilde{R}_{A}^{(2)}=\left[\frac{\langle N\rangle}{\langle N\rangle_{N U C T S T--2}}\right]_{\bar{\nu}_{N e}{ }_{N e}} .
$$

$R_{A}$ is clearly greater for $\pi^{ \pm} \mathrm{Ne}$ than for $\left(\bar{\nu}^{\prime}\right) \mathrm{Ne}$, as expected from the arguments of Chspter 2. More surprising, while $R_{A}(\operatorname{minus})$ is about 0.1 


\section{TABLE 0.2.8.}

$R_{A}$ for $\pi^{ \pm} \mathrm{Ne}$ and $\left(\bar{\nu}^{-} \mathrm{Ne}\right.$ at 30 and $64 \mathrm{GeV} / \mathrm{c}$. See text for discussion.

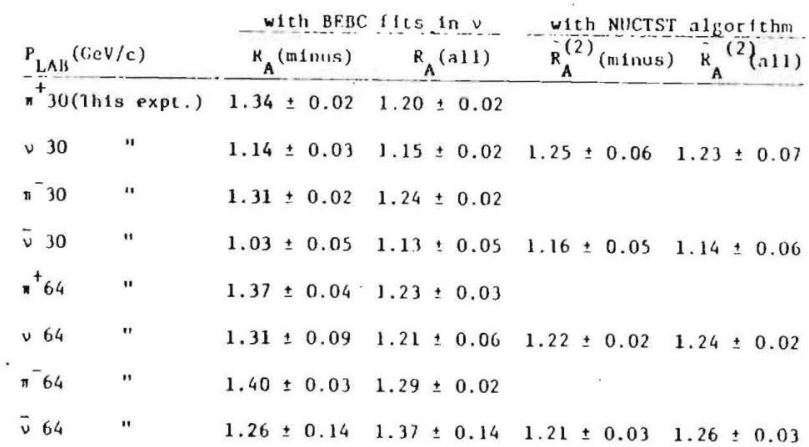

greater than $R_{A}(a l l)$ for $\pi^{ \pm} \mathrm{Ne}, R_{A}(a l l)$ is greater than or approximately equal to $R_{A}(\operatorname{minus})$ for $\left(\bar{\nu}^{\prime}\right) \mathrm{Ne}$. Näively one would expect the two definitions of $R_{A}$ to give the same results. Protons included in the shower track multiplicities for $\pi^{ \pm} \mathrm{p}$ may contribute to the difference for the $\pi^{ \pm} \boldsymbol{R}_{\boldsymbol{A}}$. For example, the average number of slow (plab $<0.8 \mathrm{GeV} / \mathrm{c}$ ) protons in $\pi^{-} p$ interactions at $16 \mathrm{GeV} / \mathrm{c}^{[0]}$ is measured to be approximately 0.25 per event. Reducing the hydrogen multiplicities by 0.25 raises $R_{A}(a l l)$ for $\pi^{ \pm} \mathrm{Ne}$ by roughly 0.04 . The larger differences in $\left(\bar{\nu}^{\prime}\right) \mathrm{Ne}$ at $64 \mathrm{GeV} / \mathrm{c}$ may arise from the fact that the BEBC fit is at the limits of the BEBC phase space in this regime, and from smaller statistics than in $\pi^{ \pm} \mathrm{Ne}$ In any case, the $\tilde{R}_{A}^{(2)}$ values, which are free from all normalization and systematic differences, agree within errors. $\tilde{R}_{A}^{(2)}$ seems to cluster strongly around $\tilde{R}_{A}^{(2)} \approx 1.23$ at these energies.

Figure 6.2.2 shows the evolution of $\boldsymbol{R}_{A}$ with energy. $\boldsymbol{R}_{A}$ is increasing rapidly for $(\bar{\nu})$ Ne and slowly for $\pi^{ \pm} \mathrm{Ne}$. This is probably because $R_{A}(\pi N e)$ has nearly saturated at these energies, while $R_{A}\left(\bar{\nu}^{\prime} N_{e}\right)$ is still approaching saturation. Clearly there must be some upper limit to the number of rescatters that can occur in the nucleus; that is, some $\langle\nu\rangle_{\text {mas }}$. Since the probability of a rescatter is roughly $1 \frac{1}{2}$ times as great in $\pi^{ \pm} \mathrm{Ne}$ than in $(\bar{\nu}) \mathrm{Ne}$, this limiting value of $R_{A}$ will be reached at lower energy for $\pi^{ \pm} \mathrm{Ne}$ $\operatorname{than}^{(-)} \mathrm{Ne}$.

6.8. Proton multiplieity distributions. 
A. Identified protons

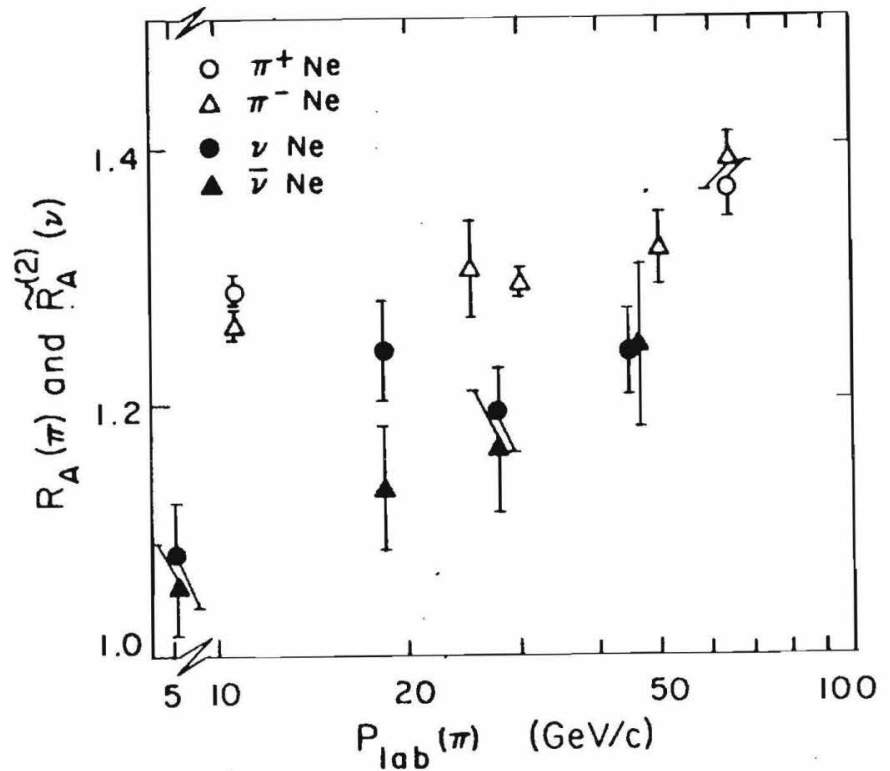

FIGURE 6.8.8.

The erolution of $R_{A}$ with energy for $\pi^{ \pm} \mathrm{Ne}$ and $\left(\bar{\nu}^{\prime} \mathrm{Ne}\right.$. All $\boldsymbol{R}_{A}$ are calculated using $\left\langle N_{p r}^{-}\right\rangle$. The $\left(\bar{\nu}^{\prime}\right)$ Ne values are $\tilde{R}_{A}^{(2)}$ (see text).
The identifled proton multiplicity distribution in E546 differs radically from the equivalent distribution in $\pi^{ \pm} \mathrm{Ne}$, and, indeed, does not have the characteristics expected for such a distribution. Since the main thrust of E548 during the scanning and measuring process was to accurately reconstruct most of the energy flow in the event, the efficiency for identified protons in experiment $\mathrm{E54B}$ is relatively low. Therefore the identifled proton distribution in E546 is considered inherently unreliable and will not be reported here. However, there is considerable evidence that the properties of events with some number $N_{p}$ of identifled protong remains unbiased in E546. That is, while the ratio $\left(N_{p}=1\right):\left(N_{p}=0\right)$ may be unreliable in $\mathbf{E 5 4 6}$, the average shower multiplicity of events with 1 proton $\langle N\rangle_{N_{p}-1}$, for example, is still comparable to the same quantity in other experiments. This is equivalent to saying that the successful identification and measurement of a proton in 2546 is totally uncorrelated to the measurement of the remainder of the event, which is an entirely reasonable statement considering the vast differences between an "identifled proton" track and any other kind of track. A check of this reveals that the ratios $\langle N\rangle_{N_{p}-0}:\langle N\rangle_{N_{p}-1}:\langle N\rangle_{N_{0}-2}$ are identical for the $\pi^{ \pm} \mathrm{Ne}$ experiment and the $\left(\bar{\nu}^{\prime}\right) \mathrm{Ne}$ experiment.

The average number of identifled protons produced at 30 and 04 $\mathrm{GeV} / \mathrm{c}$ in $\pi^{ \pm} \mathrm{Ne}$ is given in table 6.3.1. Values at $10.5^{[10]}, 25^{[11]}, 50^{[11]}$, and $200 \mathrm{GeV} / \mathrm{c}^{[12]}$ are included for comparison. The $\left(N_{p}\right)$ at $10.5 \mathrm{GeV} / \mathrm{c}$ and $200 \mathrm{GeV} / \mathrm{c}$ are considerably larger than the other values in the table; 
TABLE 6.8.1.

Average multiplicity and dispersion for identified protons $N_{p}$ in $\pi^{ \pm} \mathrm{Ne}$

\begin{tabular}{|c|c|c|c|}
\hline$P_{1 . A B}(G$ & $v / c)$ & $<\mathrm{N}>$ & ${ }_{p}$ \\
\hline$n^{+} 301$ & his expt.] & $1.31 \pm 0.02$ & $1.47 \div 0.02$ \\
\hline-30 & $"$ & $1.21: 0.02$ & $1.40 \div 0.02$ \\
\hline$x^{+} 64$ & $"$ & $1.29 \pm 0.04$ & $1.44 \pm 0.04$ \\
\hline$n-64$ & $"$ & $1.22 \div 0.04$ & $1.43 \div 0.04$ \\
\hline$n^{+} 10.5$ & {$[10]$} & $1.71 \div 0.09$ & $1.84 \div 0.09$ \\
\hline$\pi^{-}-10.5$ & $|10|$ & $1.50 \div 0.08$ & $1.77 \pm 0.08$ \\
\hline$\pi^{-} 200$ & {$[12]$} & $1.77 \div 0.10$ & \\
\hline$"-25$ & $\{11\}$ & $1.32 \leq 0.07$ & \\
\hline nso & {$[111]$} & $1.37+0.05$ & \\
\hline
\end{tabular}

excluding these, it would seem that $\left\langle N_{p}\right\rangle$ for Ne depends only on the incident particle type and not on the incident energy. This could be a saturation effect, since nuclear effects can add a maximum of 10 protons to the final state. The higher $\left\langle N_{p}\right)$ values may possibly be explained by the lower density of the $\mathrm{H}_{2}-\mathrm{Ne}$ mixture used for these experiments. A lower density allows the detection of protons with a smaller minimum momentum than that in WA51.

Figure 6.3.1 gives the multiplicity distribution for $N_{p}$. Also shown on the figure is the predicition of the Andersson model ${ }^{[1]}$, which describes the multiplicity distribution of protons in nuclear interactions.

The Andersson model can be derived from two simple assumptions. We consider the picture of nuclear scattering where there are $\nu$ (no relationship to the $\nu$ of neutrino scattering) interactions in the nucleus. In this picture we assume: 1) Each of the $\nu$ interactions are totally independent and any one interaction can be interchanged with any other, and 2) each of the $n$ protons produced at any one of the $\nu$ interactions are also independent and interchangable. From this we deduce that if the probability of producing one proton at one vertex is $x$, then the probability of two protons is $x^{2}$, and so on. The probability of something happening ( 0 protons to $\infty$ protons) must be one, so the condition

$$
a \sum_{n=0}^{\infty} x^{n}=1
$$

determines the normalization constant $a=(1-x)$, and the probability of producing $n$ protons at one vertex is $(1-x) x^{n}$.

We can calculate the average number of collisions in the nucleus from a Glauber formalism (impact parameter representation + Wood-Saxon 
nuclear density), yielding $\langle\nu\rangle$ independent of experimental results. If on the average there are $\left\langle N_{p}\right\rangle$ protons observed, then by assumption (1) the average number of protons produced at one vertex is $\left\langle N_{p}\right\rangle_{\nu-1}=\left\langle N_{p}\right\rangle /\langle\nu\rangle$.

We then solve for $\mathrm{x}$ from the equation

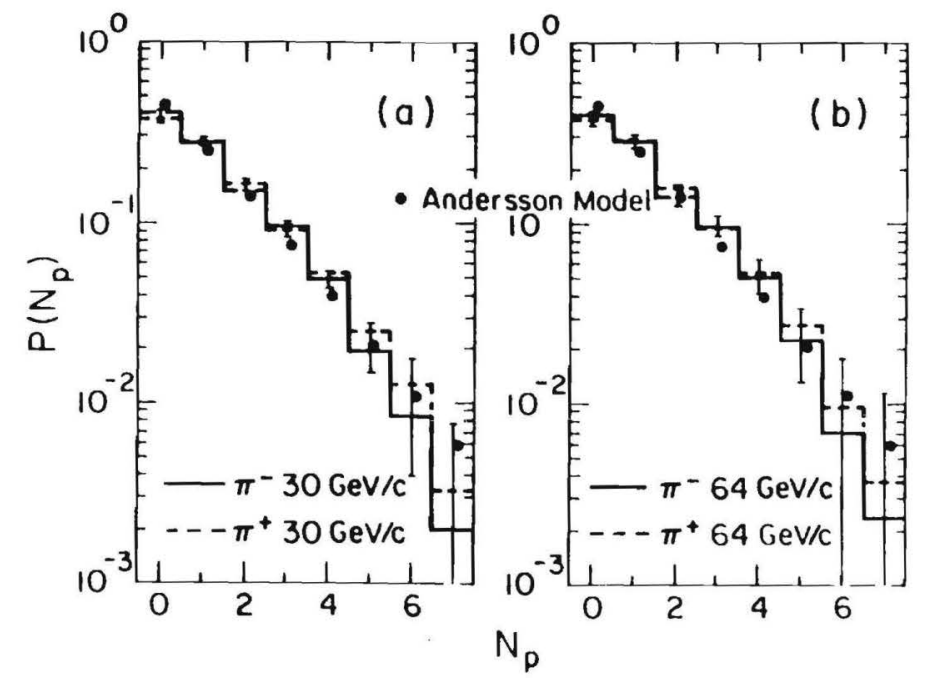

FIGURE 6.8.1.

Normslised multiplioity distribution of identified protons $\left\langle N_{p}\right\rangle .(\bullet) \pi^{ \pm} \mathrm{Ne}$ at $30 \mathrm{GeV} / \mathrm{c}$ and (b) $\pi^{ \pm} \mathrm{Ne}$ at $64 \mathrm{GeV} / \mathrm{c}$. The points show the theoretical predictions of the Andersson model ${ }^{[1]}$ (see text)

$$
\begin{aligned}
\left\langle N_{p}\right\rangle_{\nu-1} & =(1-x) \sum_{N_{p}=0}^{\infty} N_{p} x^{N_{p}} \\
& =(1-x) \frac{x}{(1-x)^{2}}
\end{aligned}
$$

to obtain

$$
P_{\nu-1}\left(N_{p}\right)=(1-x) x^{N,} \text { where } x=\frac{\left(N_{p}\right)_{\nu-1}}{1+\left(N_{p}\right)_{\nu-1}}
$$

Now if we produce $n$ total protons at $\nu$ vertices, we must make sure we count each way of distributing the protons among the vertices such that the sum is $n$ (see figure 6.3.2). Thus the probsbility for $n$ protons with $\nu$ vertices is

$$
P_{\nu}(n)=\sum_{\substack{\text { oll } \\ \text { oomb }}}\left(\prod_{i=1}^{\nu} P_{n_{1}}\right) \text { where } \sum n_{i}=n .
$$

Taking all combinations of $N_{p}$ things in $\nu$ boxes $=\left({ }_{N_{p}}^{N_{p}-1}\right)$ we arrive at the result

$$
P_{\nu}\left(N_{p}\right)=\left(\begin{array}{c}
N_{p}+\nu-1 \\
N_{p}
\end{array}\right)(1-x)^{\nu} x^{N_{p}}
$$

Given the $P_{\nu}$ we calculate the total probability by summing over all $\nu$ that are relevant:

$$
P\left(N_{p}\right)=\sum_{\nu} \Pi(\nu) P_{\nu}\left(N_{p}\right)
$$




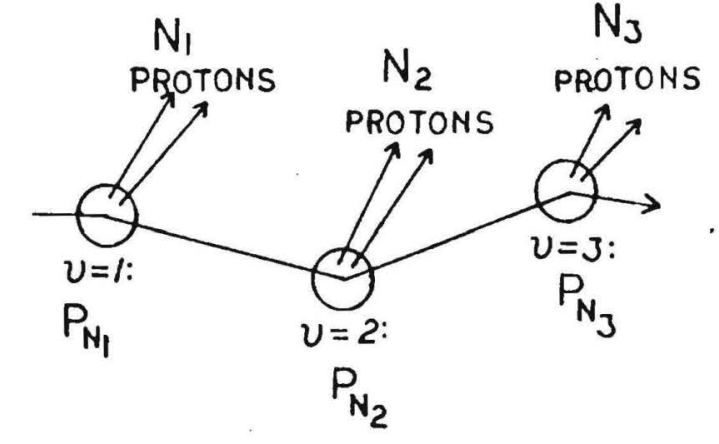

$N_{1}+N_{2}+N_{3}=N_{p}$

3 COLLISIONS

\section{FIGURE 6.8.8.}

An example of proton production in the Andersen model $[1] . P_{\mathbf{i}}=$ probability of producing $i$ protons. We must have $n_{1}+n_{2}+n_{3}=n$, ie $\sum_{i=1}^{\nu} n_{i}=n$ where $\Pi(\nu)$ is the probability of $v$ interactions, calculated from the Glauber formalism. Thus we see that the two assumptions produce a model with only one experimental parameter, $\left\langle N_{p}\right\rangle$, which only plays the role of a normalization constant.

Previously the Andersson model has been compared to data from emulsion experiments, involving a mixture of heavy and light nuclei. Since the $\pi^{ \pm}$data presented here involve only Ne nuclei, they constitute a cleaner test of the Andersson model. Using the parameters $\langle\nu\rangle=1.8, \Pi(\nu=$ 1) $=0.59, \Pi(\nu=2)=0.30, \Pi(\nu=3)=0.11,(\Pi(\nu>3)$ are ignored) and $\left\langle N_{p}\right\rangle_{\nu-1}=0.78 \pm 0.04$, the model yields the points shown in figure B.3.1. Agreement between the model and the $\pi^{ \pm} \mathrm{Ne}$ data is excellent.

\section{B. Fast (nonidentified) protons}

By using the isospin symmetry of the Ne nucleus we can deduce that the number of fast (nonidentifled) protons in the final state for $\pi^{ \pm} \mathrm{Ne}$ and $(-\bar{\nu}) \mathrm{Ne}$ is given by

$$
\left\langle N_{p}^{f}\right\rangle_{+}=\left\langle N^{+}\right\rangle_{+}-\left\langle N^{-}\right\rangle_{-}
$$

or alternatively by

$$
\left\langle N_{p}^{f}\right\rangle_{-}=\left\langle N^{+}\right\rangle_{-}-\left\langle N^{-}\right\rangle_{+},
$$

where the " + " subscript refers to either $\pi^{+} \mathrm{Ne}$ or $\nu \mathrm{Ne}$, and the "-" subscript then refers to either $\pi^{-} \mathrm{Ne}$ or $\nu \mathrm{Ne}$, respectively. The two definitions 
fair to say that current models cannot explain fast proton production.

\section{6.4. Multiplieity correlations between identified protons and} shower tracks.

As discussed in section 6.3 , the number of observed protons $N_{p}$ is directly related to the number of "effective scatterings" $\langle\nu\rangle$ in the nucleus. Since this is the case, any average multiplicity should increase monotonically with $\boldsymbol{N}_{\boldsymbol{p}}$. Indeed, figure 6.4.1 shows that $\left\langle\boldsymbol{N}_{p r}^{-}\right\rangle$rises linearly with $N_{p}$. Evidently the rate of increase is greater for $\pi^{ \pm} 64 \mathrm{GeV} / \mathrm{c}$ than for $\pi^{ \pm} 30 \mathrm{GeV} / \mathrm{c}$, indicating the slope is energy dependent. Although there is more scatter in the $\left(\bar{\nu}^{\prime}\right) \mathrm{Ne}$ points due to poorer statistics, they are also consistent with a linear rise. The $\left.{ }^{(} \bar{\nu}\right)$ Ne points are about 0.5 units below the corresponding $\pi^{ \pm} \mathrm{Ne}$ points, but their slope is consistent with being the same as that for $\pi^{ \pm} \mathrm{Ne}$.

Figure 6.4.2 shows the dispersion $D_{p r}^{-}$versus $N_{p}$. Scatter due to poor statistics in the $\mathrm{e}^{(-\bar{\nu})}$ Ne points is too severe to extract the trend of the $\left(\bar{\nu}^{\prime}\right) \mathrm{Ne}$ dats, but it would seem that although $N_{p r}^{-}$is significantly lower for $\left.{ }^{(-)}\right) \mathrm{Ne}$ than $\pi^{ \pm} \mathrm{Ne}$ at equivalent energies, $D_{p r}^{-}$is approximately equivalent. This implies that the difference shown on the $D_{p r}^{-}$vB. $N_{p r}^{-}$plot (figure 6.2.1) for $\left(\bar{\nu}^{\prime} \mathrm{Ne}\right.$ and $\pi^{ \pm} \mathrm{Ne}$, with $\left(\bar{\nu}^{\prime}\right) \mathrm{Ne}$ above $\pi^{ \pm} \mathrm{Ne}$, comes from a decrease in $N_{p r}^{-}$for $^{(} \bar{\nu}^{-} \mathrm{Ne}$, with $D_{p r}^{-}$remaining essentially constant.

$D_{p r}^{-}$, like $N_{p r}^{-}$, is linear in $N_{p}$ for $\pi^{ \pm} \mathrm{Ne}$, with the slope of $D_{p r}^{-} \mathbf{v s}$. $N_{p}$ increasing for higher energies. However, $D_{p r}^{-}$does not rise as quickly as $N_{p r}^{-}$, implying that the multiplicity distribution is becoming more "bunched" as $\langle\nu\rangle$ increases.

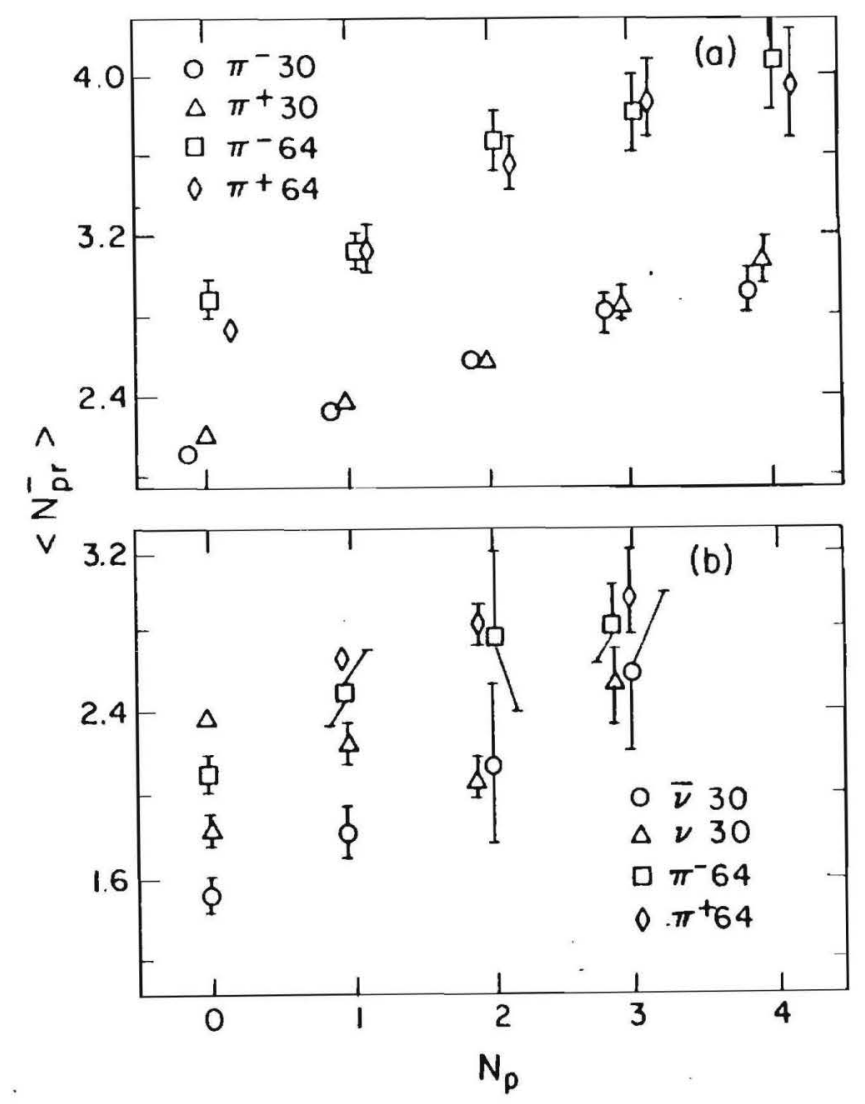

\section{FIGURE 6.4.1.}

Average multiplicity of produced negative particles $\left\langle N_{p r}^{-}\right\rangle$as a function of the identifled protons $N_{p}$. Errors are statistical only. 
133

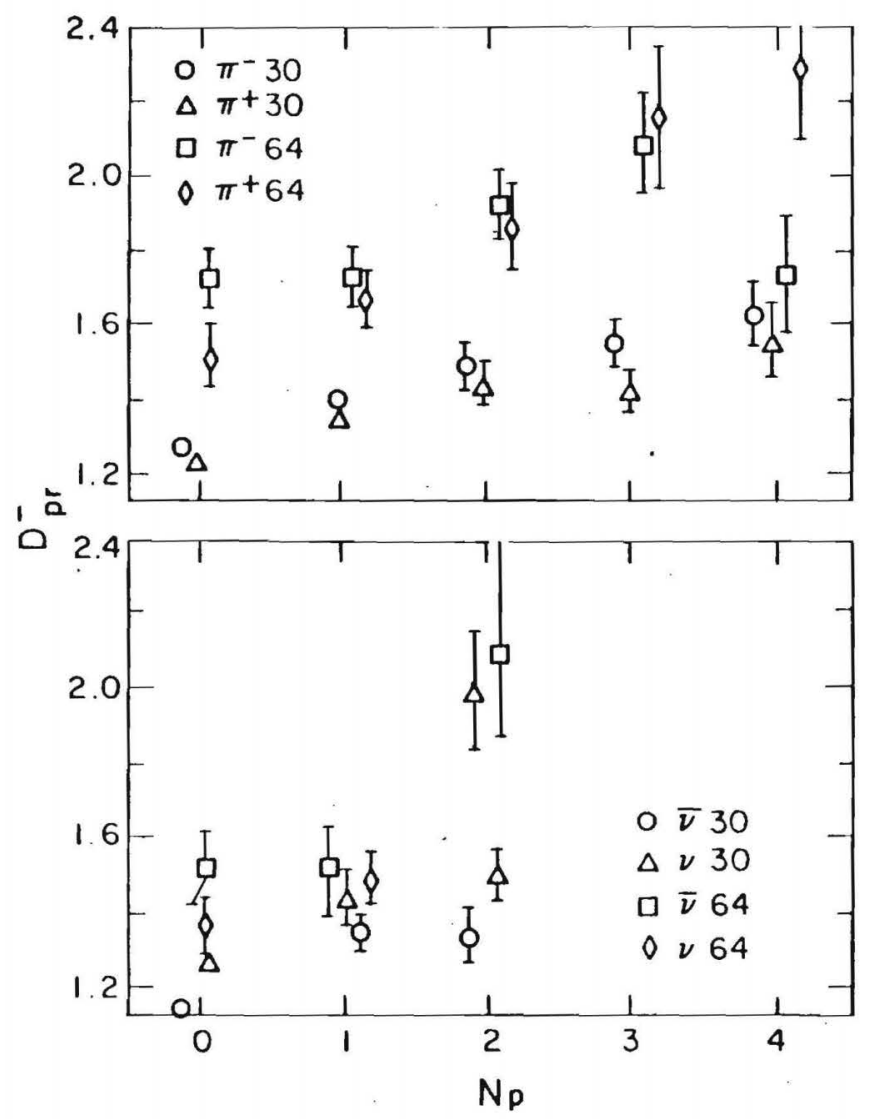

FIGURE 6.4.2.

Dispersion $D_{p r}^{-}$as a function of $N_{\mathrm{p}}$. Errors are statistical only.
134

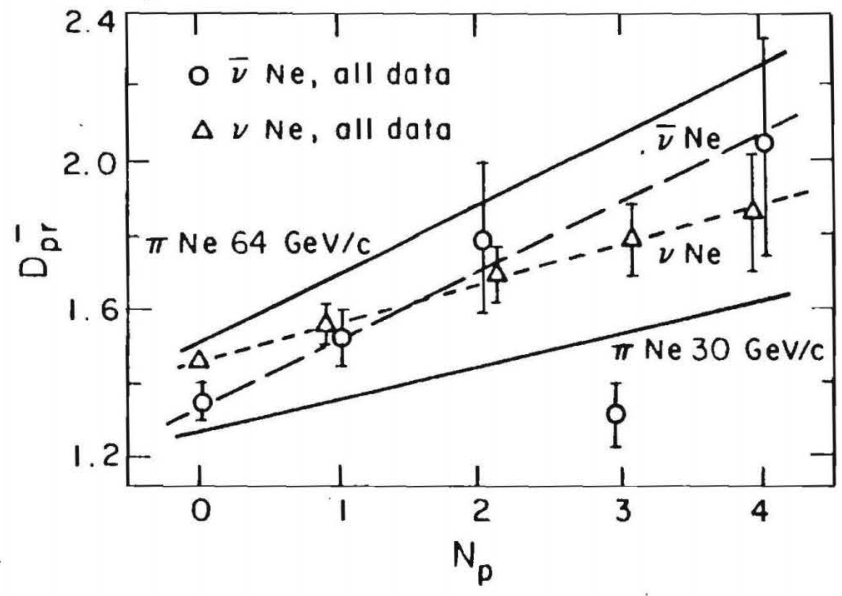

FIGURE 6.4.8.

The dispersion in $N_{p r}^{-}\left(D_{p r}^{-}\right)$for all neutrino data. The two solid lines show the linear grewth of $D_{p^{-}}^{-}\left(x^{ \pm} \mathrm{Ne}\right)$ for $30 \mathrm{GeV} / \mathrm{c}$ and $84 \mathrm{GeV} / \mathrm{e}$. The dashed lines are linear fits to the neutrino data (the $N_{p}=3$ point for $D \mathrm{Ne}$ is excluded from the fit). Errors are statistical only. 
are equivalent to first order. For convenience in reference, deffnition 6.3.1 is refered to, for example, as " $\left\langle N_{p}^{\prime}\right\rangle$ in $\pi^{+} 30 \mathrm{GeV} / \mathrm{c}^{n}$ for $30 \mathrm{GeV} / \mathrm{c}$ incident $\pi^{ \pm}$beams, and definition B.3.2 as “ $\left\langle N_{p}^{f}\right\rangle$ in $\pi^{-} 30 \mathrm{GeV} / \mathrm{c}^{\prime} . N^{+}$and $N^{-}$refer to the minimum-ionizing positive and negative tracks, respectively, with identified protons excluded. (For a more complete discussion of isospin subtraction, see section 7.5.)

Table 6.3.2 shows the average multiplicity and percentage of minimum-ionizing positive tracks for fast (nonidentified) protons in $\pi^{ \pm} \mathrm{Ne}$ and $\left(\bar{\nu}^{\prime}\right) \mathrm{Ne}$, obtained from table $\mathbf{8 . 2 . 1}$. All the values are roughly consistent except for $\nu \mathrm{Ne}$, which is high, and the $\pi^{-}$values at $10.5 \mathrm{GeV} / \mathrm{c}$ and $200 \mathrm{GeV} / \mathrm{c}$, which are slightly low. $(\bar{\nu}) \mathrm{Ne}$ tends to be slightly higher than $\pi^{ \pm}$Ne. There appears to be $\approx 0.0-0.7$ fast protons per event. This is about $20 \%$ of the positive charge multiplicity.

There will be some contribution to the average fast proton multiplicity $\left\langle N_{p}^{\text {fact }}\right\rangle$ from Kaons since $\mathrm{K}^{+}$is not the isospin partner of $\mathrm{K}^{-}$. This contribution is, however, negligible; both experiment $[10]$ and the Lund Monte Carlo calculations show any such contribution $<5 \%$ of the total charge difference at the most. In addition the observed fast protons cannot be due to baryon-antibaryon production since any such pairs are subtracted out in taking $\left\langle N^{+}\right\rangle-\left\langle N^{-}\right\rangle$.

In addition to $\pi^{ \pm} \mathrm{Ne}$ and $(\bar{\nu}) \mathrm{Ne}$, Azimov et al. ${ }^{[7]}$ estimate $\left\langle N_{p}^{f a o t}\right\rangle=$ $0.6 \pm 0.1$ for $p N e$ at $300 \mathrm{GeV} / \mathrm{c}$, consistent with the values given in table 6.3.2. This suggests the $\left\langle N_{p}^{f a s t}\right\rangle$ can depend only weakly on the incident energy or the incident particle type.

We can obtain a rough estimate of the number of fast protons expected from scattering on individual nucleons in the nucleus, ie not com-
TABLE 6.8.8.

A verage multiplicity and percentage of minimum-ionising positive tracks for fast protons for $\pi^{ \pm} \mathrm{Ne}$ and $\left.\bar{\nu}^{(}\right) \mathrm{Ne}$. Values for $\pi^{ \pm} \mathrm{Ne}$ at $10.5 \mathrm{GeV} / \mathrm{c}$ and $200 \mathrm{GeV} / \mathrm{c}$ are added for comparison.

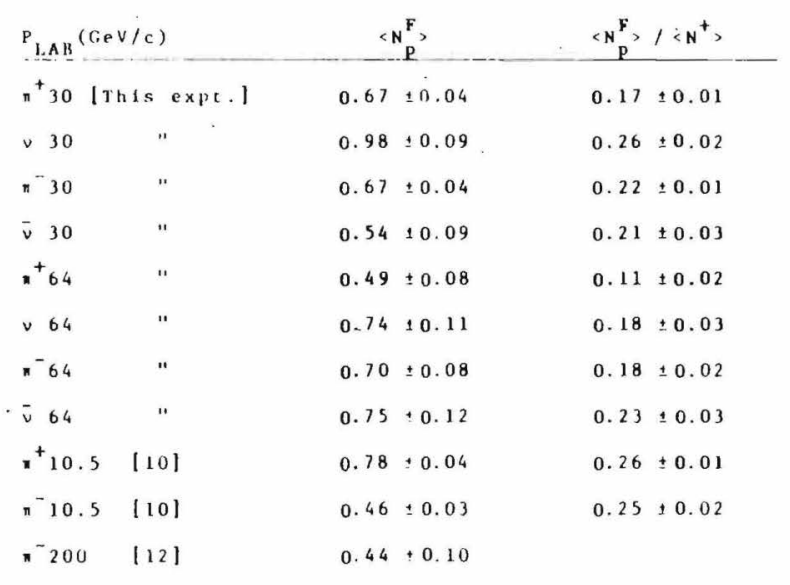


ing from nuclear effects (a more detailed study of this using the Lund Monte Carlo is given in Chapter 7). Since Ne contains equal numbers of protons and neutrons, there will be approximately 0.5 recoiling protons per interaction. The sverage number of slow protons expected is obtained using the momentum spectra of observed protons in $\pi p$ at $16 \mathrm{GeV} / \mathrm{c}^{|0|}$ and in $\pi n$ at $21 \mathrm{GeV} / \mathrm{c}^{[13]}$. Averaging these $\left\langle N_{p}\right\rangle$ values for $\pi p$ and $\pi n$ and subtracting from 0.5 yields approximately $0.33 \pm 0.05$ fast protons per event. This indicates that much of the fast proton excess is associated with production in the Ne nucleus.

Assuming that 0.33 fast protons are produced at each collision in the nucleus, then the total $\left\langle N_{p}^{\text {fast }}\right\rangle=0.33\langle\nu\rangle$. This would imply that $\langle\nu\rangle \approx$ $1.5-2.2$, which is consistent with the results given in Section 6.4. Since it is also true that $\left\langle N_{p}\right) \sim\langle\nu\rangle$, one expects that $\left\langle N_{p}\right\rangle \sim\left\langle N_{p}^{\text {fast }}\right\rangle$, ie $N_{p}$ and $N_{p}^{\text {fast }}$ should be correlated.

Such a correlation can be tested by assuming that charge symmetry also holds for each value of $N_{p}$, and computing $\left\langle N_{p}^{\text {fact }}\right\rangle$ for each $N_{p}$. Figure 6.3.3 gives $\left\langle N_{p}^{f a s t}\right\rangle$ versus $N_{p}$ for $\pi^{ \pm} \mathrm{Ne}$ and $\left(\bar{\nu}^{\prime}\right)$ Ne. The similarity of the results for + incident and - incident gives some confldence that charge symmetry can indeed be applied in this way. The $\nu(\bar{\nu})$ Ne results at $30 \mathrm{GeV} / \mathrm{c}$ show some variation; however, this is where the poorest $\left(\bar{\nu}^{\prime}\right)$ statistics occur, tending to produce variations in any case.

No significant correlation is seen in flgure 6.3.3, contradicting the hypothesis that $\left\langle N_{p}^{f a c t}\right\rangle$ and $N_{p}$ are correlated through scattering in the Ne nucleus. It is possible that such a correlation is prohibited by the limits for Ne for $(\nu)$ and $\left(N_{p}\right)_{\text {max }}$. It has been reported ${ }^{[14]}$ that larger nuclei do show evidence of a correlation between $\left\langle N_{p}^{\text {fact }}\right\rangle$ and $N_{p}$. Still, it seems
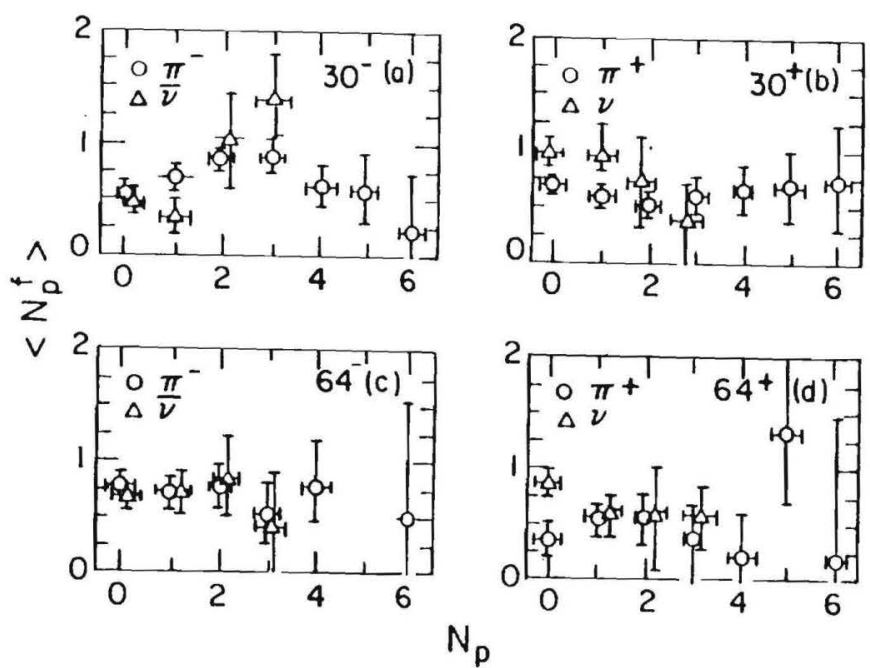

FIGURE 6.8.8.

A verage number of fast protons $\left\langle N_{p}^{\text {faot }}\right\rangle$ as a function of $N_{p}$ for $\pi^{ \pm} N e$ and $\left(\bar{\nu}^{\prime} \mathrm{Ne}\right.$. (a) $\pi^{-}(\bar{\nu}) \mathrm{Ne}$ at $30 \mathrm{GeV} / \mathrm{c},(\mathbf{b}) \pi^{+}(\nu) \mathrm{Ne}$ at $30 \mathrm{GeV} / \mathrm{c},(\mathbf{c}) \pi^{-}(\bar{\nu}) \mathrm{Ne}$ at $64 \mathrm{GeV} / \mathrm{c},(\mathrm{d}) \pi^{+}(\nu) \mathrm{Ne}$ at $64 \mathrm{GeV} / \mathrm{c}$ 
To improve the statistics for $D_{p r}^{-}\left(\left(\hat{\nu}^{-}\right)\right.$, figure 6.4 .3 plots $D_{p r}^{-}$for all $W^{2}$ in the neutrino data. The trends for $\pi^{ \pm} \mathrm{Ne}$ are shown at 30 $\mathrm{GeV} / \mathrm{c}$ and $84 \mathrm{GeV} / \mathrm{c}$. With the improved statistics an excellent linear relationship between $D_{p r}^{-}\left({ }^{-} \bar{\nu}^{\prime} \mathrm{Ne}\right)$ and $N_{p}$ is revesled. The slope for $\nu \mathrm{Ne}$ parallels that of $\pi^{ \pm} \mathrm{Ne}$ at $64 \mathrm{GeV} / \mathrm{c}$, while the $\nu \mathrm{Ne}$ slope parallels the $30 \mathrm{GeV} / \mathrm{c}$ line. Since the $\left\langle\boldsymbol{W}^{2}\right\rangle$ for $\nu \mathrm{Ne}$ is considerably higher than the $\left\langle W^{2}\right\rangle$ for $\nu \mathrm{Ne}$, and $D_{p r}^{-}$is an increasing function of the CM energy, this would seem to indicate that $D_{p r}^{-}$increases faster with $(\nu)$ for antineutrino than for neutrino (Note that $\langle\nu$ ) is a monotonically increasing function of $N_{p}$ ). However, the comparison of two data samples over large ranges of $W^{2}$ entails intrinsic difficulties of normalization which may strongly affect multiplicity distributions, so this conclusion should be considered tentative at best.

$R_{A}(x)$ and $\tilde{R}_{A}^{(2)}(\nu)$ are shown in figure 6.4.4. The $\pi \mathrm{Ne}$ data are averaged for $\pi^{+}$and $\pi^{-}$at each energy to improve statistics, since no significant differences were observed for the $\pi^{+}$and $\pi^{-}$samples. To improve statistics, all $W^{2}$ for $(\bar{\nu}) \mathrm{Ne}$ are included in the plot. The $\nu$ and $\nu$ data are averaged as the $\pi$ data for the black dots; the black triangles represent $\nu$ Ne only. $R_{A}(\pi)$ is derived from a parameterisation of $\pi p$ data ${ }^{|8|} ; \tilde{R}_{A}^{(2)}(\nu)$ uses the NUCTST algorithm - for a discussion of both, see Section 6.2.

The $\nu$ Ne points track the $\pi$ Ne points well except for the $N_{p}=4$ point, which is probably a fluctuation due to poor statistics. The $\pi 64$ $\mathrm{GeV} / \mathrm{c}$ points tend to be slightly higher than the $\pi 30 \mathrm{GeV} / \mathrm{c}$ points. The $\bar{\nu}$ data are lower than the $\nu$ data.

Berlad et al ${ }^{[18]}$ have derived the Collective Tube Model prediction for $R_{A}\left(N_{p}\right)$ (see section 2.5 for a discussion of the Collective Tube Model).

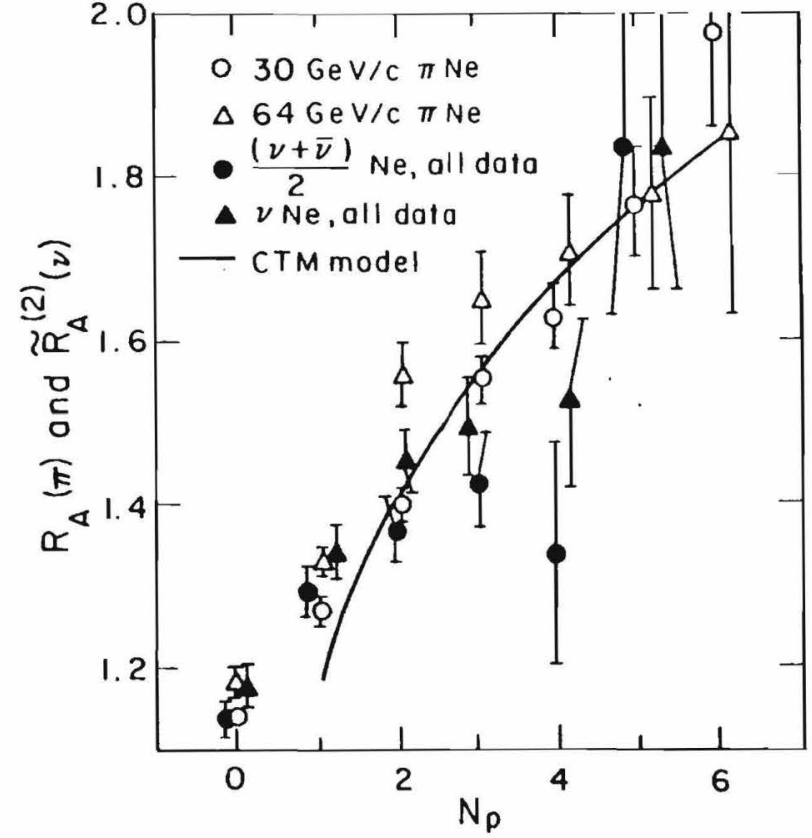

FIGURE 0.4.4.

$R_{A}(x)$ and $\tilde{R}_{A}^{(2)}(\nu)$ versus $N_{p}$ for $x^{ \pm} \mathrm{Ne}$ and $\left(\bar{\nu}^{\prime}\right)$ Ne. The $\pi$ Ne data are averaged for $\pi^{+}$and $\pi^{-}$at each energy. The $\nu$ and $D$ data are similarly averaged for the black dots; the black triangles represent $\nu$ Ne only. All $W^{2}$ for $\left(\bar{\nu}^{-}\right)$are included in the plot. $\boldsymbol{R}_{\boldsymbol{A}}(\boldsymbol{\pi})$ is derived from a parameterisation of $\pi p$ data ${ }^{|8|} ; \tilde{R}_{A}^{(2)}(\nu)$ uses the NUCTST algorithen - for a discussion of both, see Section 6.2. The solid curve is the CTM model prediction ${ }^{[15]}$. 
They assume that the average multiplicity varies with $\mathrm{CM}$ energy as $\langle n(E)\rangle \sim C E^{1 / 4}$, so that the exponent $\alpha$ in eqtn. 2.5.23 is $\alpha=1 / 4$. Then since in the CTM $\langle\nu\rangle$ is the number of nucleons in the "tube", and assuming that on the average, for $N_{p}$ protons observed, there are $[(A-Z) / Z] N_{\mathrm{p}}$ neutrons in the tube, so that the total number of nucleons in the tube is $\nu=(A / Z) N_{p}$, they derive

$$
R_{A}\left(N_{p}\right)=\left(\frac{A N_{p}}{Z}\right)^{1 / 4} .
$$

This prediction is included on figure 8.4.4. In view of the fact that this relation has no adjustable parameters, it agrees remarkably well with the data for 2 identifled protons and greater.

Given $R_{A}$ as a function of $N_{p}$, as in figure 6.4.4, the average number of interactions $\langle\nu\rangle$ can be extracted as a function of $N_{p}$ if we have a parameterization of $R_{A}$ in terms of $\langle\nu\rangle$. In view of the fact that $\left\langle N_{p r}^{-}\right\rangle$ increases linearly with $N_{p},\langle\nu\rangle$ should also increase linearly with $N_{p}$. Since many models predict a relationship of this form ${ }^{|10|}$, the parameterization used for $R_{A}$ in terms of $(\nu)$ is

$$
R_{A}=C+0.5\langle\nu\rangle,
$$

where the constant $C$ is fixed by normalization to our data as $C=0.4$.

Using this parameterization, $\langle\nu\rangle$ is obtained as a function of $N_{p}$ in figure 6.4.5. $\langle\nu\rangle$ rises linearly with $N_{p}$ from about 1.5 for $N_{p}=0$ to about 3.0 for the highest values of $N_{p}$.

The $C_{p r}^{k-}$ versus $N_{p}$ are shown in flgure 6.4.6 for $x^{ \pm} \mathrm{Ne}$ (the $t^{\left(-\nu^{\prime}\right.} \mathrm{Ne}$ data are not shown due to poor statistics). These moments show a small but systematic decrease with increasing $N_{p}$. Similar behavior has been

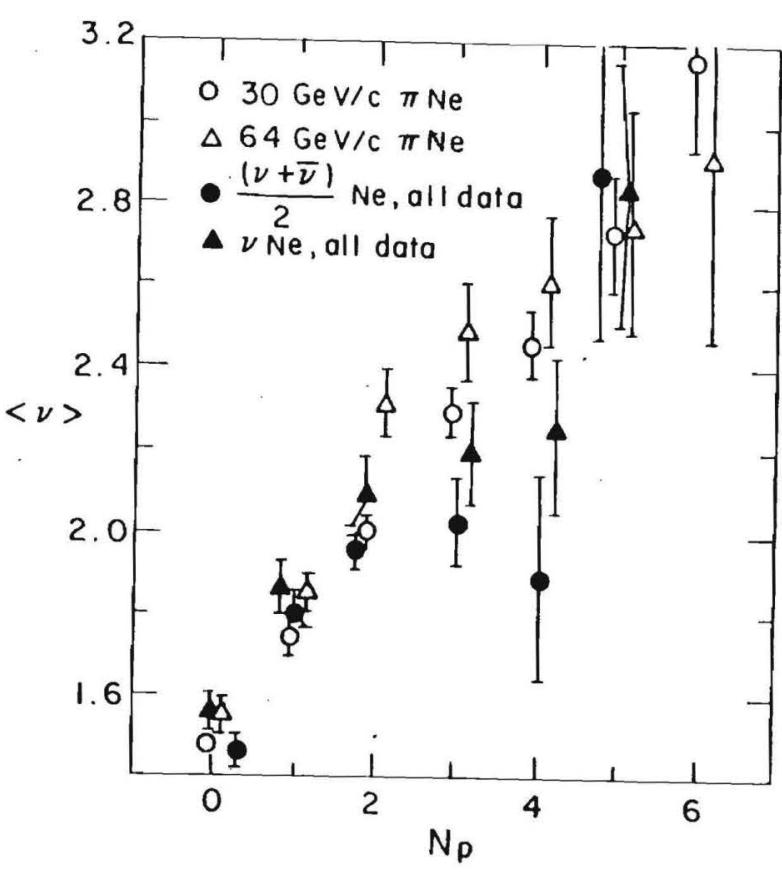

FIGURE 6.4.5.

A rerage number of "effective" collisions $(\nu)$ versus $N_{p}$. Results are derived using the relation $R_{A}=0.4+0.5(\nu)$ (see text) and the $R_{A}$ shown in figure 6.4.4. 


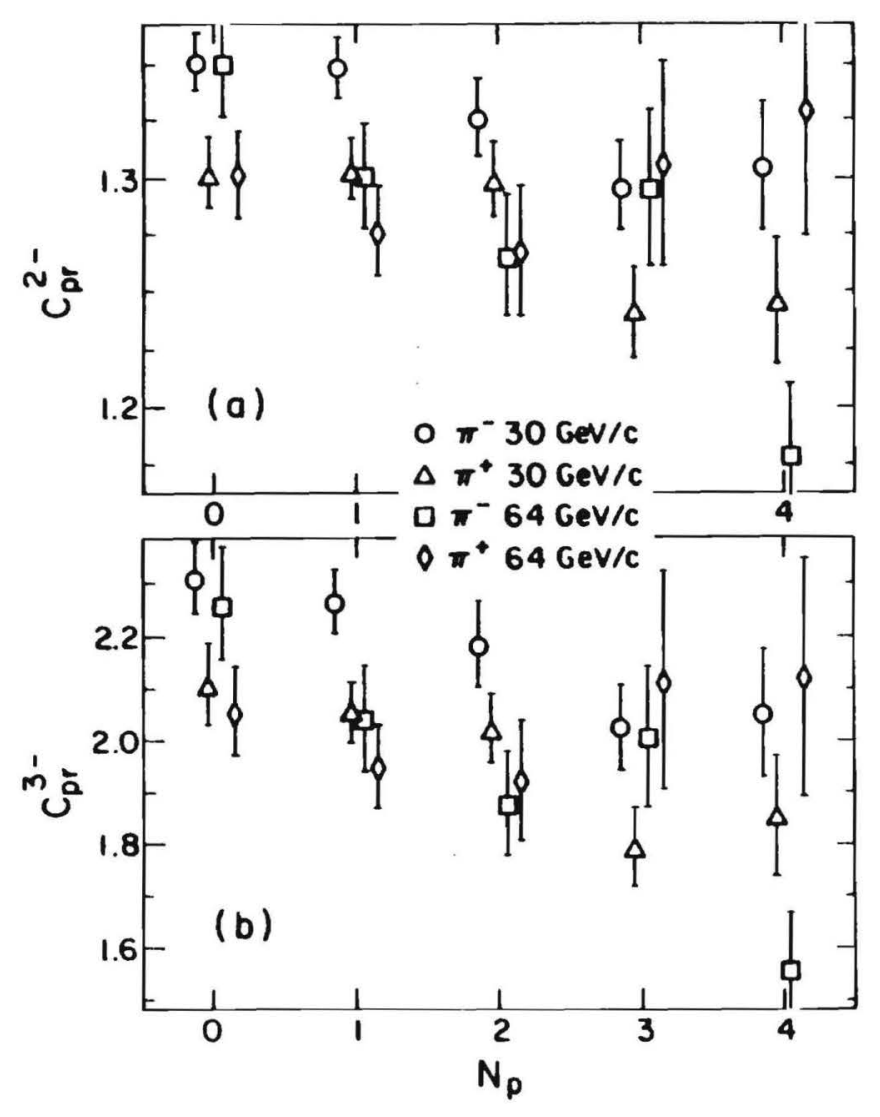

TIQURE 0.4.6.

Normalised moments $C_{\mathrm{pr}}^{2-}$ and $C_{\mathrm{pr}}^{8-}$ for $N_{\mathrm{pr}}^{-}$for $\pi^{ \pm} \mathrm{Ne}$ as a function of N. $\left(\bar{\nu}^{\prime}\right.$ Ne data not shown due to poor statistics.) observed in $p N e$ interactions at $300 \mathrm{GeV} / \mathrm{c}^{|\eta|}$. Since $\langle\nu\rangle$ is a linearly increasing function of $N_{p}$, and the $C_{p r}^{k-}$ moments by deffition determine the shape of the KNO function in $N_{p r}^{-}$, this suggests that multiple scattering in the nucleus produces a slight violation of KNO scaling.

\section{6.5. KNO Sealing.}

In $1972 \mathrm{Kobs}$, Nielsen, and Olesen ${ }^{[2]}$ proposed a scaling law for multiplicity distributions based on the idea of Feynman scaling. The KNO scaling law states that

$$
\begin{aligned}
P_{n}(s) & =\frac{1}{\langle n\rangle} \phi\left(\frac{n}{\langle n\rangle}\right)+o\left(\frac{1}{\langle n\rangle^{2}}\right) \text { where } \\
P_{n}(s) & =\frac{\sigma_{n}(s)}{\sigma_{\text {inel }}(s)} \\
s & =\text { CM energy squared } \quad \text { and } \\
\psi & =\text { a univeral function. }
\end{aligned}
$$

KNO scaling is only expected to hold to order $1 /(n)^{2}$, or equivalently to $O\left(1 /(\ln s)^{2}\right)$. This result bolds for any given type of produced particle, or any given mixture of types of produced particles. Further, this scaling law should be "invariant under resonance decay" (that is, still hold valid even when including decay products of resonances in a given particlespecies multiplicity), provided all resonsnce production contributing to a particle species grows with energy in the same way. In short, the ratios of resonance types produced (for example, $\left(n_{p}\right) /\left(n_{\Delta}\right)$ ) should remain constant with energy, which will be the case if the energy is high enough to avoid threshold effects. This is an important exception, because at typical 
experimental energies some baryonic resonances are still in the threshold regime and this may lead to KNO violations.

The form of the KNO scaling function $\phi(z)$, where $z=n /(n)$, is to be determined by experiment. In the limit $\rightarrow \infty$, the absolute moments $C^{k}$, defined as $C^{k}=\langle n\rangle^{k} /\left\langle n^{k}\right\rangle$, determine $\psi(z)$ via

$$
\int_{0}^{\infty} z^{k} \psi(z) d z=C^{k}
$$

KNO speculated that $\phi(z)$ was independent of incident particle type; however, they based this speculation on results of a Regge-type model with pomeron exchange. While such a model may have its regions of applicability, it is not viewed as fundamental in the modern QCD approach, and so there is no strong reason to believe KNO scaling should be independent of particle type. Experiment has since shown that different scattering systems do indeed follow different KNO scaling curves (in the sense of $e^{+} e^{-}$vs. hadron-nucleon, say). The initial quark content of the system would seem to be the important factor here.

In general, KNO scaling is obeyed surprisingly well considering the relatively low $s$ and $(n)$ arailable to experimentalists. It would seem that KNO scaling is obeyed to the level of $10-20 \%$, but evidently not at the level of $3 \%$. Different schemes exist for improving this discrepancy: One may exclude the energy necessary for baryon conservation in hadronnucleon interactions, and consider only an "effective" energy $E_{e f f}$ available for particle production; since events coming from a diffractive process are a special case and clearly do not scale with energy, one may wish to exclude these events; since leading particles may be viewed as carrying some memory of the original "quarkiness" of the system, these may also be excluded $^{[17]}$. In general, KNO enthusiasts attempt to remove any "nonthermal" processes from the multiplicity, where by non-thermal I mean any process not following some type of statisticsl distribution- for example, Bose-Einstein statistics. Not surprisingly, such approaches do indeed increase agreement with KNO scaling. It is my feeling, however, that such deviations as are seen from KNO scaling are still within theoretical (in the sense of approximating a sum by an integral) and experimental uncertainties (systematic and other difficulties), so attempting corrections reveals little new knowledge.

What determines the underlying nature of the scaling distribution $\psi(z)$ itself is unknown. The interesting question is whether $\psi(z)$ is determined by fundamental properties of high energy physics or whether $\psi(z)$ is determined only by statistics - equipartition of energy into final states, say. Due to the wide range of scattering systems where KNO applies, one might be tempted to say that a statistical mechanism has more to do with the result than any detailed dyamical result. Carruthers and Shih ${ }^{|18|}$ have recently noticed that the generalized Bose-Einstein distribution (or negative binomial distribution) given by

$$
\begin{aligned}
P_{n}^{(k)} & =\frac{(n+k-1) !}{n !(k-1) !}\left(\frac{\langle n\rangle / k}{1+(n) / k}\right)^{n} \frac{1}{(1+(n) / k)^{k}} \text { with } \\
k & =3 \text { to } 4 \text { (for KNO scaling) }
\end{aligned}
$$

does a good job of describing KNO scaling (here $k$ is the number of "cells"). This distribution describes photoelectron counts from thermal sources, coherent light transmitted through liquids at their critical point, and, evidently, the probability of finding $\boldsymbol{n}$ galaxies in a $Z$ wicky cluster. Carruthers and Shih therefore suggest that the generalized Bose-Einstein 
distribution describes counting from any physical system with $\boldsymbol{k}$ cells with rapidly varying emitting fields at the emitting surface (ie, Gaussian distributed).

They also note that there exists a smooth interpolation between Poisson counting and KNO scaling (Poisson distributions do not scale) This implies there may be some Poisson component in KNO distributions, hidden by the acaling properties of KNO. In any case, the argument for a general statistical origin of KNO scaling would seem to be persuasive.

For low $(n)$, violations of KNO scaling also arise from the approximation of a sum in computing the $C^{k}$ by an integral. Even given a universal $\psi(z)$, in the experimental case where we choose from a finite set $n \in$ $\left\{1 \ldots N_{\max }\right\}$ then when we evaluate

$$
\left\langle n^{k}\right\rangle=\frac{1}{\langle n\rangle} \sum_{n=1}^{N_{m a *}} \psi\left(z \equiv \frac{n}{\langle n\rangle}\right) n^{k}
$$

we evaluate $\psi(z)$ at different $z$ and the $C^{k}$ moments may not be constant. Further obscuring any information contained in KNO scaling plots is the fact that we evaluate $z$ over a very limited range (for small, ie accessible,

$(n))$. Thus if $P(n)$ is any fairly smoothly varying function, when we transform to $z$ we would expect to see something like "scaling" in any case.

Figure 0.5.1 shows the KNO scaling curve for $\pi^{ \pm} \mathrm{Ne}$ at 30 and 64 $\mathrm{GeV} / \mathrm{c}$ and for all the ${ }^{(-\bar{\nu})} \mathrm{Ne}$ data.

Figure 6.5.2 compares KNO results for the nuclear, non-nuclear, and mixed algorithm $($ NUCTST $=+2$, NUCTST $=-2, N U C T S T=0)$ in $(-)$. If observed nuclear effects come strictly from reinteractions in $\mathrm{Ne}$, with each interaction being more or less like $\bar{\nu}^{\prime} p$, then KNO scaling will not be affected by nuclear effects; alternatively, if there is a modification of the
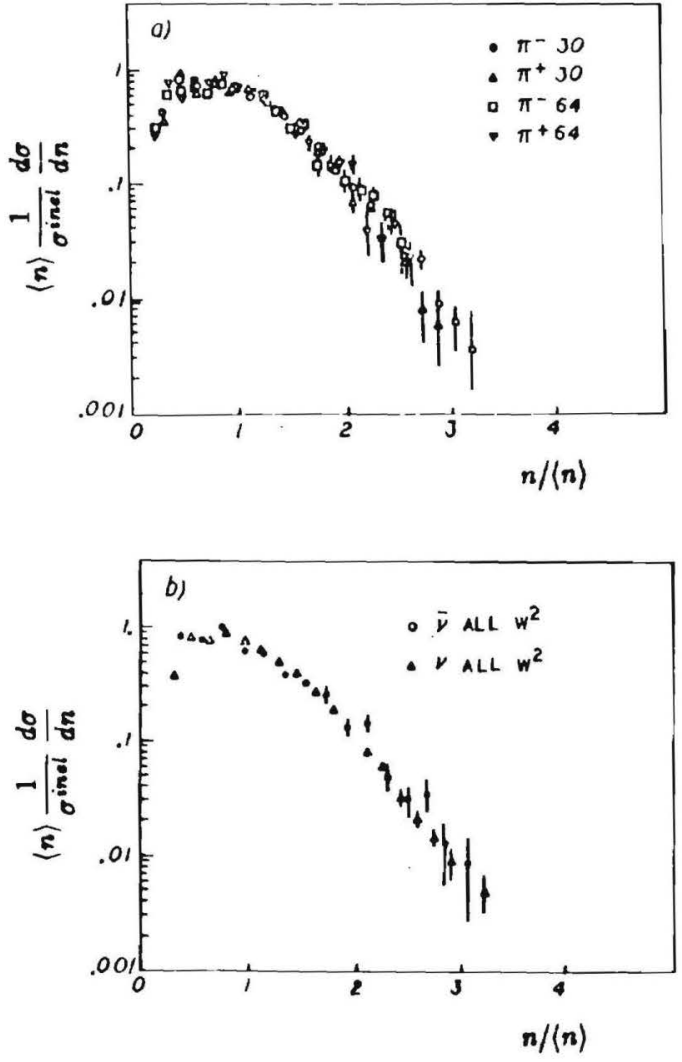

FIGURE 6.8.1.

The KNO scaling distribution for shower partlcles comparing different hadronic $\mathrm{OM}$ energies. (a) $\pi^{ \pm} \mathrm{Ne}$ at 30 and $64 \mathrm{GeV} / \mathrm{c}$, and (b) $\nu$ and $D$ for the full range of $W^{2} . \nu$ and $D$ are weighted to have equivalent $W^{2}$ distributions. 

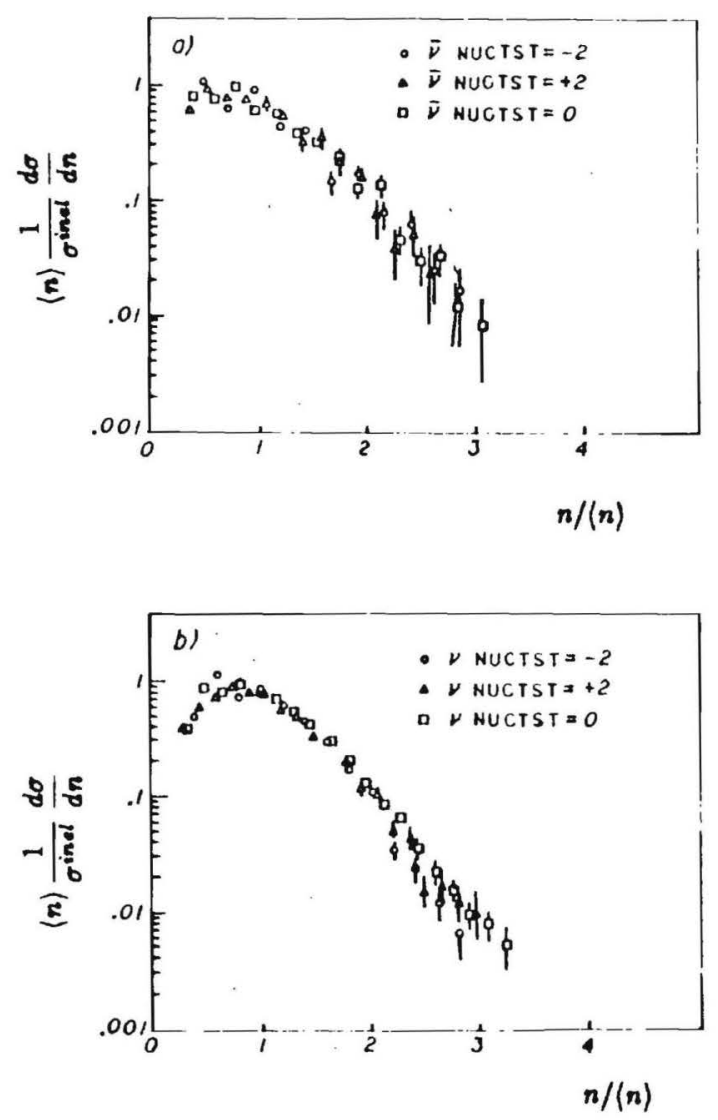

FIGURE 6.5.8.

The KNO scaling distribution for shower particles for nuclear, non-nuclear, and total algorithms. (a) $\nu \mathrm{Ne}$ and (b) $\nu \mathrm{Ne}$. quark structure functions in nuclei as opposed to nucleons, as the EMC effect ${ }^{[10]}$ suggests, then the KNO distributions could vary. However, if KNO is an essentially statistical effect, then modiffcation of the structure functions would not alter KNO. The figure shows that all curves are compatible. Furthermore, KNO plots of $\nu$ and $\bar{\nu}$ data at different $\boldsymbol{W}^{2}$ show no evidence of any variation with $W^{2}$ in $\left(\vec{\nu}^{\prime} \mathrm{Ne}\right.$.

Finally, figure 6.5.3 compares the KNO scaling curves for $\pi^{ \pm} \mathrm{Ne}$ and $\left.{ }^{(}{ }^{-}\right) \mathrm{Ne}$ at equivalent hadronic $\mathrm{CM}$ energies, and also with ${ }^{(-)} \mathrm{Ne}$ evaluated over the full experimental $W^{2}$ range. Again all curves are consistent with following a universal curve.

\section{6.6. Features of multiplielty distrlbutlons as funetions of lep-} tonle and hadronle event variables in $(\bar{\nu})$.

As discussed in Chapter 5, the experimental dynamics of creating a neutrino beam necessitate that a relatively broad range of neutrino energies will be present in the beam. Also the virtual momentum transfer to the target in deep inelastic lepton-nucleus scattering may vary over a considerable dynamic range. As a result E546 covers a broad range of $W^{2}$, equivalent to $s$ in hadronic scattering, and also a broad range of $Q^{2}$, the absolute value of the mass of the spacelike four-momentum transfer.

It is perhaps more accurate to view $\mathbf{E 5 4 6}$ as an ensemble of experiments carried out under identical conditions, but with different virtual $W^{+}$and $W^{-}$beams. Given this ensemble, it becomes possible to study experimental results over the full $\mathbf{E 5 4 6}$ range of hadronic system CM energies. 
147
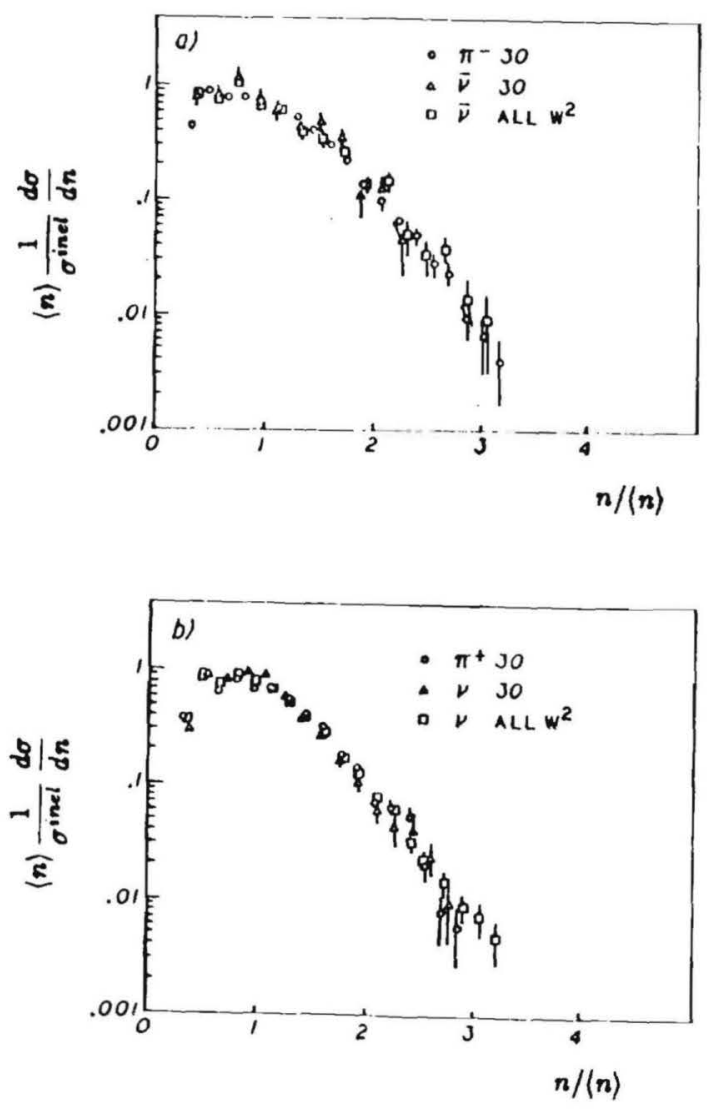

TIGURE 6.5.8.

Comparison of the KNO scaling curves for shower particles between $\left(\bar{\nu}^{\prime} \mathrm{Ne}\right.$ and $\pi^{ \pm} \mathrm{Ne}$. (a) $\nu \mathrm{Ne}$ and $\pi^{-} \mathrm{Ne}$ and (b) $\nu \mathrm{Ne}$ and $\pi^{+} \mathrm{Ne}$.
148

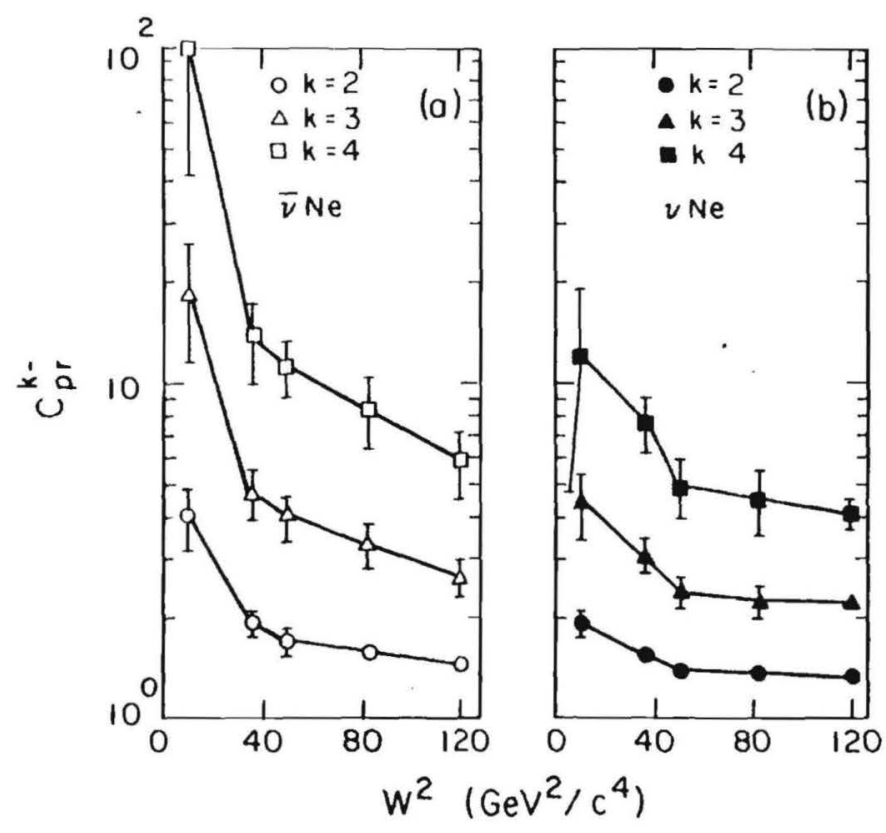

FIGURE 0.6.1.

$C_{p r}^{k-}$ moments for $k=2,3,4$ as a function of $W^{2}$. (a) $D N e$ and (b) $\nu \mathrm{Ne}$. The $C_{\mathrm{pr}}^{k-}$ are extrapolated to zero bin width. 
In figure 6.8.1, the behavior of the $C_{\mathrm{pr}}^{k-}$ moments with $W^{2}$ is shown.

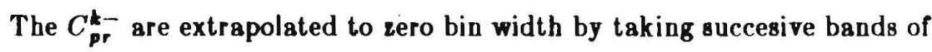
successively smaller width and extrapolating linearly to zero width. All

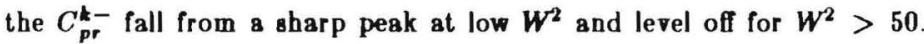
As discussed in section 6.5, this would indicate some violation of KNO

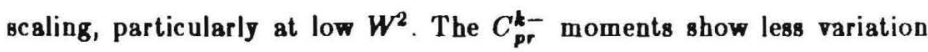
in $\nu$ than in $\nu$. This may be due to the incident $W^{-}$in $\nu$ tending to spread the negative charge produced over a larger region of the accessible phase space (that is, to larger values of Feyman $\mathrm{x}$ ) than is the case in $\nu$, where there is no incident negative charge. $C_{\mathrm{pr}}^{2-}$ seems to be approaching an asymptotic value of approximately 1.5 at high $W^{2}$ for both $\nu$ and $\nu$. The other $C_{\mathrm{pr}}^{\mathrm{k}-\mathrm{are}}$ still showing some variation, although $C_{\mathrm{pr}}^{3-}$ may be approaching the asymptotic value of about 2.3-2.5.

Other moments in $N_{\mathrm{pr}}^{-}$are shown in figure 6.6.2 as functions of $W^{2}$. $D_{p r}^{-}$is identical for both $\nu$ and $\nu$ and is rising as $\ln \left(W^{2}\right)$, just as $N_{p r}^{-}$ does and as we expect from the existence of the relationship $D_{\mathrm{pr}}^{-}=A+$ $B \cdot N_{p r}^{-}$for all energies. The skewness in $N_{p r}^{-}, \gamma_{p r}^{-}$, is essentially constant at $\gamma_{\overline{p r}}^{-} \approx 1.0$, with the $\nu \mathrm{Ne}$ values perhaps slightly above this. Since the skewness is a measure of how much the distribution is "skewed" to one side or the other, with positive values of $\gamma_{p r}^{-}$meaning that the "tail" of the distribution is for greater $N_{p r}$, this indicates that as $N_{p r}^{-}$grows with $W^{2}$ it is essentially scaling upwards, retaining a tail of constant relative area.

$f_{p r}^{2--}$ is the integral of the two-particle distribution function in $N_{p r}^{-}$ ie $f_{\mathrm{pr}}^{2--}=\int C\left(y_{1}, y_{2}\right) d y_{1} d y_{2}$, and provides a measure of the "clumpiness" of produced negative particles in rapidity space $\left(f_{\mathrm{pr}}^{2--}=0\right.$ for a Poisson

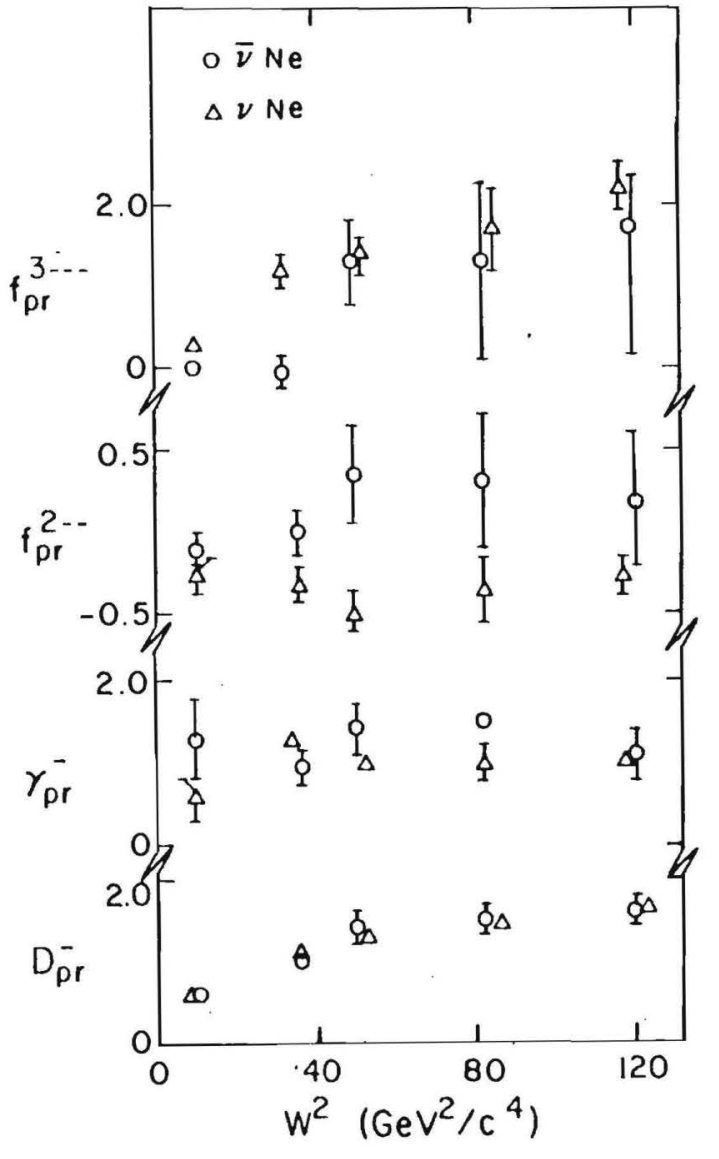

FIGURE 6.6...

$W^{2}$ dependence of $D_{p r}^{-}, \gamma_{p r}^{-}, f_{p r}^{2--}$, and $f_{p r}^{8---}$ in $\bar{\nu}^{-}$Ne. The momente are extrapolated to zero bin width. 
distribution). Similarly $f_{\mathrm{pr}}^{8---}$ is the integral of the three-particle rapidity distribution function $C\left(y_{1}, y_{2}, y_{3}\right) . f_{p r}^{2--}$ is negative at low $W^{2}$ for both $\nu$ and $\nu$, but becomes convex and positive for $\nu$, while becoming concave and remaining negative for $\nu$. This parallels the behavior of $f_{p r}^{2--}$ in $\left(\bar{\nu}_{p}|b|\right.$ Evidently the net negative charge carried into the interaction by the $W^{-}$ in $\nu \mathrm{Ne}$ is enough to provide a correlation in $N_{p}^{-}$, where no such correlation is created in $\nu \mathrm{Ne}$. This would show up as a clustering of negative charge in the projectile region of rapidity space for $D$, whereas no such clustering would be present in $\nu$. This is also consistent with the behavior of the $C_{p r}^{k-}$ moments discussed above.

$f_{\mathrm{pr}}^{3---}$ also appears to increase as $\ln \left(W^{2}\right)$ with $\nu$ and $D$ following the same trend, as in the case of $D_{p r}^{-}$. Since it is hard to imagine an effect that would have this property in both $\nu \mathrm{Ne}$ and $\nu \mathrm{Ne}$, this is probably only a manifestation of phase space constraints: It is harder to distribute three particles uniformly in a small phase space than to have some clumping of the three. If $N_{\text {pr }}$ grows more rapidly with $W^{2}$ than $y_{\max }$ does, where $V_{\text {max }}$ is the maximum possible rapidity for a given $W^{2}$, then $f_{p r}^{3---}$ will just increase as $N_{\mathrm{pr}}^{-}$. That is, $f_{\mathrm{pr}}^{8-\cdots-} \sim \ln \left(W^{2}\right)$.

Tables 6.6.1, 6.6.2, and 6.6.3 give the results of fitting $\left\langle N^{\text {ch }}\right\rangle,\left\langle N^{-}\right\rangle$, and $\left\langle N^{+}\right\rangle$to the form $(N)=a+b \cdot \ln \left(W^{2}\right)$. Table 6.8.1 gives results of a fit of this form for $\left\langle N^{c h}\right\rangle$ to a number of different scattering systems for comparison; tables 6.6.2 and 6.6.3 in $\left(N^{-}\right)$and $\left(N^{+}\right)$in $\left(\bar{\nu}^{\prime}\right.$ Ne give comparisons only $\mathrm{td}^{(} \bar{\nu}^{\prime} p$.

The bin width chosen for these fits is $1 \mathrm{GeV}^{2}$ and a smoothing algorithm consisting of a running average has been used to smooth fluctuations. The degree of smoothing is indicated by "width $=n_{f}$ ", where $n_{f}$ is
TABLE 6.6.1.

Results of fits to the form $\left\langle N^{\text {ch }}\right\rangle=a+b \cdot \ln \left(W^{2}\right)$ for various scattering systems. 〈Nohower $\rangle$, which excludes identifled protons, is also shown for E546.

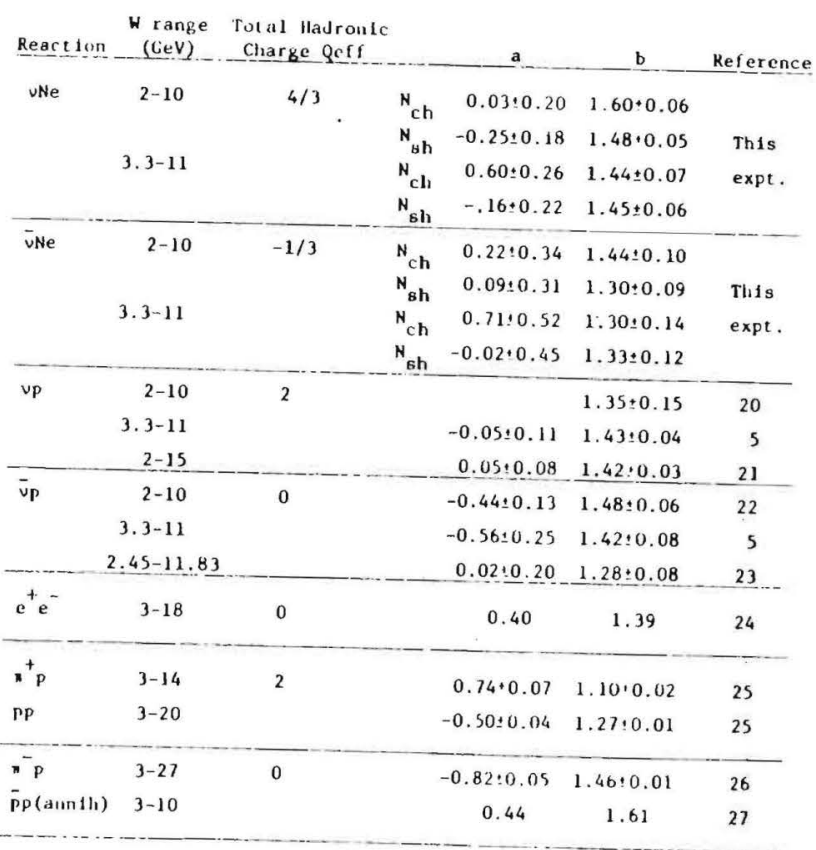




\section{TABLE 6.0.8.}

Results of fits to the form $\left(N^{-}\right)=a+b \cdot \ln \left(W^{2}\right)$ for $\left(\bar{\nu}^{\prime}\right) \mathrm{Ne}$ and $\left(\bar{\nu}^{\prime} p\right.$.

\begin{tabular}{|c|c|c|c|c|}
\hline Reaction & & W Range & $-a$ & b \\
\hline$v \mathrm{Ne}(w 1 \mathrm{dth}$ & $-2)$ & $2-10$ & $-1.11 \pm 0.08$ & $0.78 \div 0.02$ \\
\hline$v \mathrm{Ne}(w 1 \mathrm{dth}$ & $-5)$ & & $-1.06 \pm 0.05$ & $0.77 \div 0.02$ \\
\hline UNe(w1dth & $-2)$ & $3.3-11$ & $-1.04 \pm 0.12$ & $0.76 \div 0.03$ \\
\hline$v \mathrm{Ne}(w 1 \mathrm{dth}$ & $-5)$ & & $-1.02 \pm 0.08$ & $0.76 \div 0.02$ \\
\hline vp & {$[s]$} & 3. 3-11 & $-1.03 \pm 0.06$ & $0.72 \div 0.02$ \\
\hline ṼNe(w1dth & $-2)$ & $2-10$ & $-0.24 \pm 0.23$ & $0.72+0.07$ \\
\hline vine (w1dth & $-5)$ & & $-0.22 \pm 0.15$ & $0.72: 0.05$ \\
\hline VNe(w1Ath & $-2)$ & $3.3-12$ & $-0.43 \pm 0.34$ & $0.76 \pm 0.09$ \\
\hline vine (w1dth & -5) & & $-0.39 \pm 0.23$ & $0.76=0.06$ \\
\hline vp & {$[5]$} & 3. $3-11$ & $-0.28 \div 0.12$ & $0.71 \div 0.04$ \\
\hline
\end{tabular}

TABLE 6.6.8.

Results of fits to the form $\left\langle N^{+}\right\rangle=a+b \cdot \ln \left(W^{2}\right)$ for $\left(\bar{\nu}^{-}\right)$se snd $\bar{\nu}^{-} p$.

\begin{tabular}{|c|c|c|c|c|}
\hline Keacilo & & W Range & a & b \\
\hline VNe (width & $=2)$ & $2-10$ & $0.79 \pm 0.20$ & $0.71 \div 0.06$ \\
\hline vNe(wldth & $=5)$ & & $0.81 \div 0.13$ & $0.71 \div 0.04$ \\
\hline$v \mathrm{Ne}(w / d t h$ & $=2)$ & $3.3-11$ & $0.87 \pm 0.23$ & $0.69 \pm 0.06$ \\
\hline VNe (width & $=5)$ & & $0.88 \pm 0.15$ & $0.69+0.04$ \\
\hline up & [s] & $3.3-11$ & $0.95 \div 0.06$ & $0.73 \pm 0.02$ \\
\hline ine (width & $-2)$ & $2-10$ & $0.36 \div 0.25$ & $0.55 \pm 0.08$ \\
\hline vNe(width & .5) & & $0.34 \pm 0.17$ & $0.57 \pm 0.05$ \\
\hline $\bar{u}_{\text {Ne }}($ Wideh & -2) & $3.3-11$ & $0.41 \pm 0.36$ & $0.53 \pm 0.10$ \\
\hline vNe(uldth & $-5)$ & & $0.38 \pm 0.24$ & $0.56 \div 0.06$ \\
\hline vp & [s] & $3.3-11$ & $0.28 \pm 0.10$ & $0.72 \pm 0.03$ \\
\hline
\end{tabular}


the smoothing folding factor. This smoothing algorithm works as follows: A $W^{2}$ smoothing width, $\Delta W^{2}$, is chosen independent of the number of bins in the original histogram of $(N)$ versus $W^{2}$, provided only that $\Delta W^{2}>$ the original bin width. Since in this case the original bin width was 1 $\mathrm{GeV}^{2}, \Delta W^{2}$ is equivalent to the folding factor $n_{f}$, where $n_{f}$ is the number of bins of the original histogram "folded" into a smoothed bin. If $W_{\text {center }}^{2}$ is the central value of a bin in the histogram, that bin is accumulated if

$$
\left|W_{\text {eenter }}^{2}-W_{\text {event }}^{2}\right| \leq\left(\Delta W^{2}\right) / 2 \text {, }
$$

where $W_{\text {event }}^{2}$ is the $W^{2}$ of a given event.

Thus a single event actually appears over the width $\Delta W^{2}$ in the histogram, giving a folding factor of $\Delta W^{2} /$ bin width. (The folding factor is the number of times a single event contributes to the histogram). This effectively averages nearest neighbors in the histogram but does not distort the histogram shape, if we are assuming that all variations are only statistical fluctuations around a function that changes slowly with respect to the fluctuations. This is certainly the case here, since $\langle N\rangle$ varies only as $\ln \left(W^{2}\right)$

Near the boundaries of the histogram the effective folding factor will be smaller and a slight distortion will occur because averaging will be in one direction only: upward for the low edge and downward for the high edge. However these "edge effects" are compensated for by the increased error bars due to the lower effective folding factor of these edge bins, providing appropriate weighting for the fit.

In table 6.6.1, values are given for $\left(\bar{\nu}^{-}\right) \mathrm{Ne}$ for both $N^{\text {ch }}$ and $N^{\text {ohower. }}$ $N^{\text {ch }}$ counts all charged tracks, including identifled protons. $N^{\text {ehower }}$ counts only "shower" tracks (identifled protons are excluded) and so is a better quantity to compare to $\left({ }_{\nu}\right) \mathrm{Ne}$ if one wants to exclude nuclear boil-off protons and other low energy protons. The fit for the data in E546 extends over two $W$ ranges: $2-10 \mathrm{GeV}$ and $3.3-11 \mathrm{GeV}$, in order to compare with similar energy ranges in other experiments. The fits for $\mathbf{E 5 4 6}$ have a folding factor $n_{f}=5$ in table 6.8.1. Comparison of fits with different folding factors indicates that $a$, the intercept, increases by about 5$10 \%$ for large $n_{f}$, and that the slope $b$ is quite stable under changes in $n_{f}$.

Comparison of $N^{\text {ohower }}$ in $\left(\bar{\nu}^{)}\right.$Ne with the corresponding values ${ }^{\left[{ }^{[b}\right]}$ in

$(\bar{\nu})$ shows that the two are similar. This might seem surprising, since one expects higher multiplicities from nuclear rescattering, until the effective net charge $Q_{\text {efs }}$ involved in the reaction is taken into account. Since the $W^{+}$produced in $\nu$ Ne charged current only couples to $I_{3}=-\frac{1}{2}$ quarks and the ocean should be symmetric between $p$ and $n, \nu$ reactions are twice as likely to occur on $n$ than on $p$. Therefore $Q_{\text {eff }}$ for $\nu \mathrm{Ne}$ is $4 / 3$ instead of 2 in $\nu p$, and so multiplicity multiplication is indeed evident.

Table 8.8.2 for fits of $\left(N^{-}\right)=a+b \ln \left(W^{2}\right)$ shows that the slope $b$ is about 0.05 greater for both $\nu \mathrm{Ne}$ and $\bar{\nu} \mathrm{Ne}$, compared to $\left(\bar{\nu}^{\prime}\right)$. The intercept $a$ is evidently comparable in both systems. In contrast, the slopes given in table 6.6.3 for $\left(N^{+}\right)$are evidently lower for $(-\bar{\nu})$ Ne than for $\bar{\nu}^{-} p$, significantly lower in the case of $\bar{\nu}$ incident. This is reasonable since the $Q_{\text {eff }}$ of table 6.8.1 is relatively negative for both $\nu \mathrm{Ne}$ compared to $\nu p$ and for $\nu \mathrm{Ne}$ compared to $\nu p$. Some interplay is visible between $a$ and $b ; b$ should be considered the more strongly determined parameter.

There have been some report that multiplicities are correlated with the leptonic variables $Q^{2}, x, y$, as well as the hadronic rariable $W$. First, 


\section{TABLE 6.6.4}

Results of fits to the form $\left\langle N^{\text {ohowar }}\right\rangle=a+b \cdot Q^{2}$ for $(\bar{\nu})$ Ne. The fits are for $0 \leq Q^{2} \leq 40$ and for $W$ ranges $2 \leq W \leq 5,5 \leq W \leq 8$, and $8 \leq \boldsymbol{W} \leq 11$.

\begin{tabular}{|c|c|c|c|}
\hline Reaction & W Range & a & b. \\
\hline vNe (width $=1$ ). & $2-5$ & $4.2 \pm 0.2$ & $0.04 \div 0.04$ \\
\hline WNe(w1dth -2$)$ & & $4.2 \div 0.2$ & $0.05 \div 0.03$ \\
\hline$v \mathrm{Ne}(\mathrm{w} / \mathrm{dth}-1)$ & $5-8$ & $6.2 \pm 0.2$ & $-0.02 \pm 0.01$ \\
\hline UNe (w1dth $=2)$ & & $6.1 \pm 0.1$ & $-0.01 \leq 0.01$ \\
\hline WNe (w1dth $=1)$ & $8-11$ & $7.1 \pm 0.2$ & $-0.01 \div 0.01$ \\
\hline vNe (w1dth $=2)$ & & $7.1 \pm 0.2$ & $-0.01 \div 0.01$ \\
\hline $\bar{\nu}_{\mathrm{Ne}}(w 1 \mathrm{dth}=1)$ & $2-5$ & $4.0 \pm 0,4$ & $0.09 \div 0.08$ \\
\hline $\bar{v} \mathrm{He}($ w1dth $=2)$ & & $4.0 \pm 0.3$ & $0.04 \pm 0.06$ \\
\hline V̈ne (w1deh $=1$ & $5-8$ & $6.0 \pm 0.3$ & $-0.09 \pm 0.02$ \\
\hline vNe (w1dth $=2)$ & & $6.0 \pm 0.2$ & $-0.08 \leq 0.02$ \\
\hline VNe $($ wIdth $=1)$ & 8-11 & $7.0 \pm 0.5$ & $-0.07 \div 0.03$ \\
\hline VNe (wideh - 2$)$ & & $6.9 \pm 0.4$ & $-0.04: 0.02$ \\
\hline
\end{tabular}

such a leptonic correlation does not take into account kinematic correlations between the event variables (discussed in Section 7.2); Second, there is no evidence of any such correlation of $(N)$ with leptonic rariables in E548. Results of fits of the form $\left\langle N^{\text {ohower }}\right\rangle=a+b \cdot Q^{2}$ for three $W$ ranges $(2 \leq W \leq 5,5 \leq W \leq 8$, and $8 \leq W \leq 11)$ in $\left(\hat{\nu}^{\prime}\right) \mathrm{Ne}$ are presented in table 8.6.4. $Q^{2}$ was taken over the range $0 \leq Q^{2} \leq 40$; results are given for both unsmoothed distributions $\left(n_{f}=1\right)$ and minimally smoothed distributions $\left(n_{f}=2\right)$.

From the table it is evident that all slopes are consistent with $\boldsymbol{b}=\mathbf{0}$, and the more events entering into the $Q^{2}-W^{2}$ slice, the more strongly the slope is determined to be 0 . In conclusion, there is no evidence of any correlation of multiplicities with leptonic event parameters in $(\bar{\nu}) \mathrm{Ne}$ in our experiment. 


\section{Notes for Chapter}

1. B. Andersson, I. Otterlund, and E. Stenlund, Phys. Lett. 73B 343 (1978); B. Andersson et al., "A Monte-Carlo Simulation of Distributions of Recoiling Protons in High Energy Hadron-Nucleus Reactions", LU-TP-80-13 (1980); E. Stenlund and I. Otterlund, Reactions", LU-TP-
LUIP 8106 (1981)

2. Z. Koba, H.B. Nielsen, snd P. Olesen, Nucl. Phys. B40, 317 (1972)

3. M. Derrick et al., Phys. Rev. D17, 1 (1978)

4. see A. Wroblewski, "Proceedings of the IIIrd Multiparticle Symposium”, 140 (Zakapana 1972); Acta Physica Polonica B4, 857 (1873)

5. H. Grässler et al., Nucl. Phys. B223, 269 (1983)

6. D.J. Miller and R. Nowak, Lett. Nuov. Cim. 13, 39 (1975)

7. S.A. Azimov et al., Phys. Rev. D23, 2512 (1981)

8. A.K. Wroblewski, "Topologic Cross Sections in $\pi p$ Interactions", Univ. of Washington, Visual Techniques Laboratory, Physics Note PHY-90 (1982)

9. Unpublished data of the Aachen-Berlin-Bonn-CERN-CracowHeidelberg-Warsaw collaboration

10. W.M. Yeager et al., Phys. Rev. D16, 1294 (1977)

11. B.S. Yuldasher et al., Acta Phys. Pol. B9, 513 (1978)

12. W.D. Walker, " $\pi$ Neon Collisions at High Energy, High Energg Physics 1980", XX Intl. Conf., Madison, Wis., USA, edited by L. Durand, L. Pendram, 77 (New York, Amer. Inst. Phys., 1981)
13. H. Abramowicz et al., Z. Phys. C-Particles and Fields 7, 199 (1981)

14. G. Bohm et al., "On the Correlations between Slow and Fast Protons in Hadron-Nucleus Interactions", contribution to the High Energy Physics Conference (Brighton, UK, 1983)

15. G. Berlad, A. Dar, and G. Eilam, Phys. Rev. D13, 161 (1976); Y. Afek, G. Berlad, A. Dar, and G. Eilam, TECHNION-PH-76-87 (1976); S.A. Azimov et al., Phys. Lett. 73B, 339 (1978)

16. see section 2.5. See also P.M. Fishbane and J.S. Trefl, Phys. Lett $51 B, 139(1974)$

17. for example, see reference 4. See also A. Buras et al., Phys. Lett. 47B, 251 (1973)

18. P Carruthers, Phys Lett 114B, 189 (1982); P Carruthers and C.C. Shih, Phys. Lett. 127 B, 242 (1983); also P. Carruthers, "Hadronic Multiplicity Distributions: Example of a Universal Stochastic Mechanism", talk given at Los Alamos Natl. Laboratory, Los Alamos, NM, USA

19. R.G. Arnold et al., "Measurements of the A-dependence of DeepInelastic Electron Scattering From Nuclei”, SLAC-PUB-3257 (1983)

20. J. Bell et al., Phys. Rev. D19, 1 (1979)

21. M. Derrick et al., Phys. Rev. D25, 624 (1982)

22. D. Zieminska et al., Phys. Rev. D27, 47 (1983)

23. S. Barlag et al., Z. Phys. C11, 283 (1982)

24. K. Fialkowski, "A User's Note on $e^{+} e^{-}$and $\nu p$ A rerage Multiplicities" (Feb. 1982)

25. E. Albini et al., Nuov. Cim. 32A, 101 (1976)

2B. R. Barloutad et al., Nucl. Phys. B178, 285 (1980) 
27. R.M. Roberson et al., Phys. Rev. D21, 3064 (1980)

\section{CHAPTER T}

\section{KINEMATIC DISTRIBUTION RESULTS}

\subsection{Chapter overvlew.}

This chapter deals with various inclusive distributions, the effects of nuclear reinteractions, and their comparison between $(\bar{\nu}) \mathrm{Ne}$ and $\pi^{ \pm} \mathrm{Ne}$. The varisble ylab, the laboratory rapidity, is used extensively to avoid uncertainties in the Center of Mass correction. Other inclusive varisbles used are $\boldsymbol{p}$, the magnitude of the three-momentum $\mathbf{p} ; p_{T}$, the momentum transverse to the incident beam momentum (in neutrino this is $\mathbf{q}$ ); $\boldsymbol{x}_{\boldsymbol{F}}$, or Feynman $x$, the fraction of maximum possible longitudinal momentum a particle carries; and $z$, the fraction of maximum possible energy a particle carries. The rapidity is the Lorentz velocity parameter, that is, rapidities add, unlike normal velocities.

Section 7.2 compares the $W^{2}, Q^{2}$, and $\nu$ distributions of events 
that show evidence of nuclear reinteractions, events that show no such evidence, and the full data sample. The NUCTST algorithm classifies about $1 / 3$ of all events as "nuclear", and there is no deviation in the nuclear events from the nonnuclear events for these distributions. There is some indication that nuclear events exceed nonnuclear events at low $W^{2}$ (except at the lowest $W^{2}$ ), which would be expected. The deviation at lowest $W^{2}$ may be a kinematic limitation of the NUCTST algorithm. Also studied are correlations between the event variables in the neutrino data. In particular, a very tight correlation between $\boldsymbol{W}^{2}$ and $\nu$ in our data has the interesting implication that the $\gamma$ to the hadronic $\mathrm{CM}$ is $\gamma_{c} m \approx$ $0.65 W$ for all the E546 data.

Section 7.3 compares inclusive distributions in $\left(\bar{\nu}^{-} \mathrm{Ne}\right.$ and $\pi^{ \pm} \mathrm{Ne}$. A theorem by Adler ${ }^{[1]}$ has established that the combination of massless neutrinos, CVC (Conserved Vector Current) and PCAC (Partically Conserved Axial Current) are enough to guarantee that in the $Q^{2} \rightarrow 0$ limit, final state systems for $\left(\bar{\nu}^{\prime}\right.$ (anything) and $\pi^{ \pm}$(anything) are identical for equivalent hadronic CM energies. This section studies how they differ as $Q^{2}$ grows from 0 . Most striking is an excess of tracks at high $y_{c m}$ and $x_{F}$ for $\pi^{ \pm} \mathrm{Ne}$ relative to $\left(\bar{\nu}^{\prime} \mathrm{Ne}\right.$; this is interpreted as a leading particle effect for $\pi^{ \pm} \mathrm{Ne}$ not present in $\left(\bar{\nu}^{\prime} \mathrm{Ne}\right.$. Other differences are also seen. Differences in the $p_{T}$ distribution are unlikely to come from nuclear rescatter effects, as is explained in the text.

Section 7.4 studies $R_{A}$ as a function of $y$ in $\nu \mathrm{Ne}$ and $\nu \mathrm{Ne}$ using the NUCTST algorithm. $R_{A}$ indicates that there is a depletion of tracks at high rapidity (relative to ${ }^{(} \hat{\nu}^{\prime}$ (nucleon)) and an excess at low rapidity, an effect expected in string and formation-time models, as explained in section 7.4. However this depletion occurs only for low $W^{2}$, which is where we expect nuclear reinteraction effects to be important. Asymptotically (ie in the double limit $W^{2} \rightarrow \infty$ and $Q^{2} \rightarrow \infty$ ) there is no difference between nuclear and nucleon scatter; that is, $R_{A} \rightarrow 1$.

Section 7.5 is a study of unidentified proton $\left(p_{\text {fast }}\right)$ production. Using an isospin subtraction technique explained in the section, rapidity distributions are derived for the $p_{\text {faot }}$ tracks in both $\pi^{ \pm} \mathrm{Ne}$ and $\left(\bar{\nu}^{\prime}\right) \mathrm{Ne}$, and compared between the two systems. The distributions agree well. These are added to the identified proton rapidity distribution to obtain the total proton distribution. The $\boldsymbol{p}_{\text {faet }}$ distributions are compared with predictions of the LUND model ${ }^{[2]}$, compared between nuclear and nonnuclear events, and examined as functions of $W^{2}$. There is evidence in the distributiona for a separation between protons produced at the primary vertex and protons coming from nuclear reinteractions. The evolution of the $p_{\text {faot }}$ distribution with $W^{2}$ is interpreted as a phase-space effect, since the maximum possible rapidity grows with $W^{2}$ in the same way.

\subsection{General KInematic Dlstrlbutlons and Correlatlons between Kinematle Variables.}

The question arises whether events that show evidence of nuclear effects ( NUCTST $=+2)$ show any different trend in the basic kinematic event variables than all events taken as a whole $(N U C T S T=q)$ or those events that are classified as non-nuclear events $($ NUCTST $=-2)$. Figure 7.2.1 shows the trend of the three classes of events as a function of $W^{2}$ in $\nu \mathrm{Ne}$ and $\nu \mathrm{Ne}$ and figure 7.2.2repeats this for $Q^{2}$. These plots also serve 
165
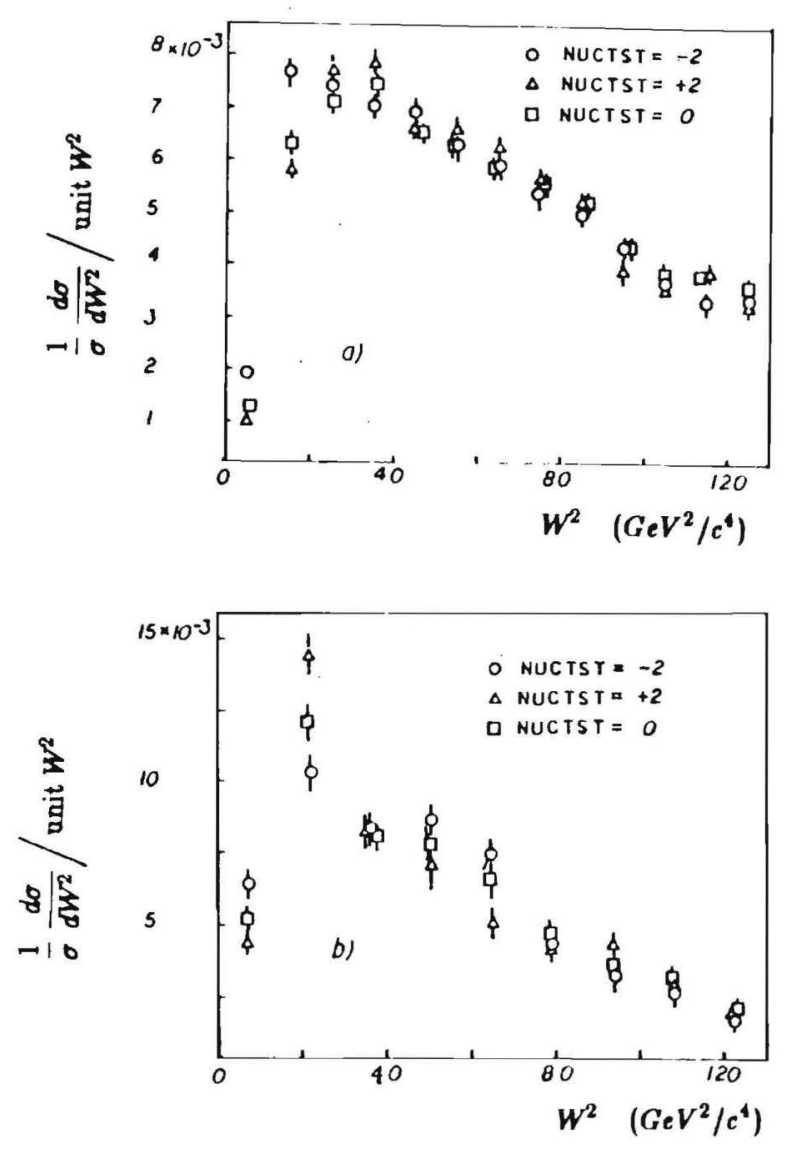

FIGURE ๆ.ฯ.1.

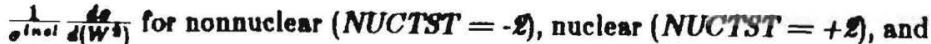
all events $(N U C T S T=g)$. (a) $\nu \mathrm{Ne}$ and (b) $D \mathrm{Ne}$.
166
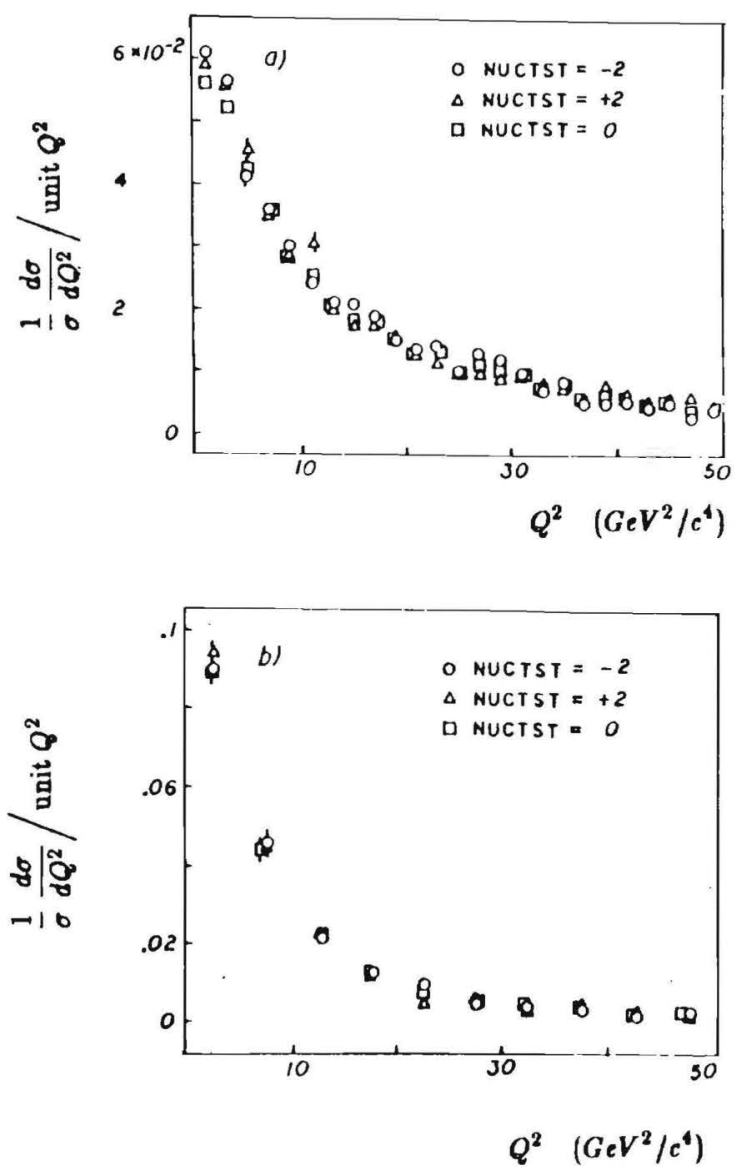

FIGURE 7.4.1.

$\frac{1}{a^{\text {inat }}} \frac{d^{\circ}}{d\left(Q^{2}\right)}$ for nonnuclear (NUCTST $\left.=-2\right)$, nuclear $($ NUCTST $=+2)$, and all events $(N U C T S T=G$ ). (a) $\nu \mathrm{Ne}$ and (b) $D \mathrm{Ne}$. 
to show the basic E546 distributions of $W^{2}$ and $Q^{2}$ (the NUCTST $=0$ points).

It is clear from these figures that there is no difference in the $W^{2}$ or $Q^{2}$ dependence of the three categories. Some dispersion does develop at low $W^{2}$ with non-nuclear events rising over nuclear-type events; this may, however, be due to low CM energies constraining particle production in such a way that multiplicities must fall the in $N U C T S T=-2$ category. For example, at $W^{2}=20$ there is only about $2 \mathrm{GeV}$ for each hemisphere in the CM. Subtracting the minimal rest mass for the baryon in the backwards hemisphere where we expect to see strong nuclear effects leaves only about $1 \mathrm{GeV}$ to be split between particle production and kinetic energy, so we are unlikely to produce more than, say, 1 pion.

Conversely, if we accept this splitting at low $W^{2}$ as real (since it occurs in both $\nu \mathrm{Ne}$ and $\nu \mathrm{Ne}$ at roughly the same magnitude and there is no evidence for a similar effect in $Q^{2}$ ), then it is in disaccord with expectations of either string or formation time models.

For example, in formation time models, a "formation time" $\Delta t$ must elapse before a rescatter can occur (for a discussion, see chapter 2). From the uncertainty principle we expect $\Delta t \sim 1 / E$, where $E$ is the relevant energy involved. Thus for large $E$ we would expect to see few nuclear rescatters since most produced particles will leave the nucleus before $\Delta t$ elapses. Since we would expect $E \sim W^{2}$, we would expect to see nuclear effects enriched, not depleted, at low $W^{2}$. Also we would not expect a correlation with $Q^{2}$ in this model. Similar arguments apply for string models. (For more on this point, see Section 7.4).

Figure 7.2.3 shows the ratio of nuclear-type events to nonnuclear-ty pe

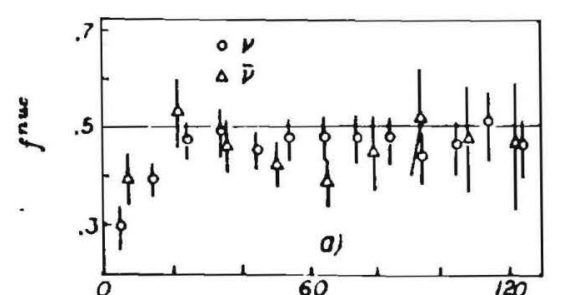

$W^{2}\left(G e V^{2} / c^{4}\right)$

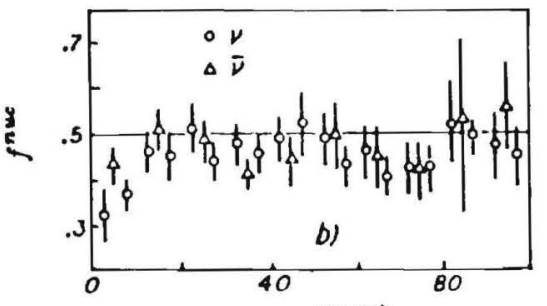

$\nu(\mathrm{GeV})$

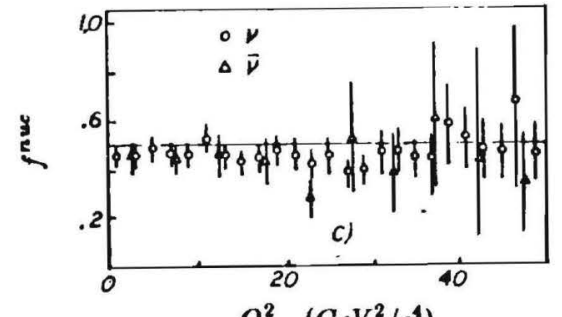

$Q^{2}\left(G e V^{2} / c^{4}\right)$

FIGURE 7.8.8.

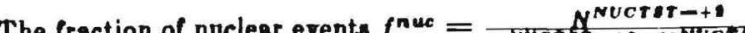
and $D N e$ versus (a) $W^{2},(b) \nu$, and (c) $Q^{2}$. 
events plus nuclear-type events,

$$
f^{\text {nue }} \equiv \frac{N^{N U C T S T-+2}}{N^{N U C T S T-+2}+N^{N U C T S T--2}} .
$$

$f^{n u c}$ is given as a function of $W^{2}, \nu$, and $Q^{2}$. $f^{\text {nuc }}$ is consistent with being $\frac{1}{2}$ throughout all ranges, except for very low values of $W^{2}$ and $\nu$ ( $W^{2}$ and $\nu$ are highly correlated; see below). The NUCTST algorithm essentially divides the E546 events into NUCTST $=+2:$ NUCTST $=-2$ : unassignable in the ratios 1:1:1, ie about $1 / 3$ of all events show evidence of nuclear effects. This is large compared to the canonical value of 10-20\% nuclear effect events; however as many as $\approx \frac{1}{2}$ of the NUCTST $=+2$ events may be classified as such because of having more than 1 identified proton, and many of these events may show no other evidence of nuclear effects. In other words, the disintegration of the Ne nucleus can be largely decoupled from the original deep inelastic scatter and come mainly from the removal of a nucleon from the nucleus.

To fully understand the properties of neutrino events as functions of the basic event rariables $W^{2}, Q^{2}, x_{B J}, y$, and $\nu$, it is important to understand the correlations among these variables. In particular, a functional dependence on one variable may only be a reflection of a correlation with another variable. From the definition $W^{2} \equiv(q+p)^{2}$, where $q$ and $p$ are respectively the four-vectors of the virtual momentum transfer and target, it immediately follows that

$$
W^{2}=-Q^{2}+m_{p}^{2}+2 \nu m_{p}
$$

Then from the definitions of the remaining event parameters it follows that knowledge of any three of the parameters $E_{\nu}, x_{B J}, y, W^{2}, Q^{2}, \nu$ is

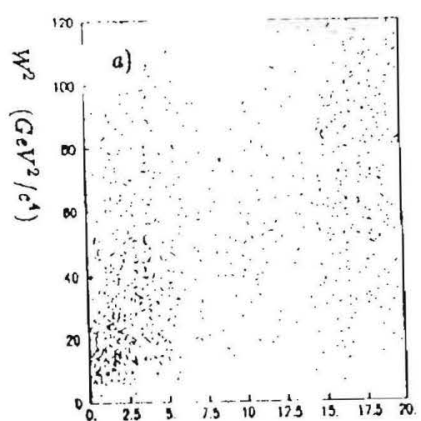

$Q^{2}\left(\mathrm{GeV}^{2} / \mathrm{c}^{4}\right)$

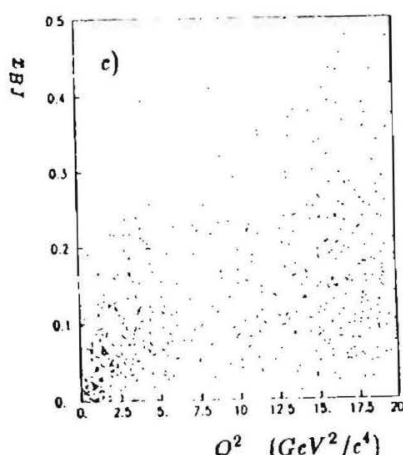

$Q^{2}\left(G c V^{2} / c^{4}\right)$

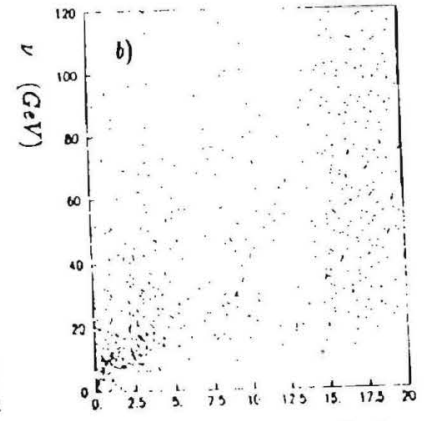

$Q^{2} \quad\left(\mathrm{GeV}^{2} / \mathrm{c}^{4}\right)$

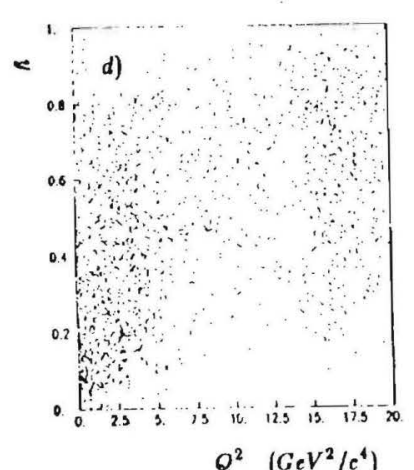

$Q^{2}\left(G e V^{2} / c^{4}\right)$

\section{FIGURE 7.8.4}

Proportional dot plots showing kinematic correlations. (See text.) (a) $Q^{2}$ and $W^{2}$, (b) $Q^{2}$ and $\nu_{1}$ (c) $Q^{2}$ and $\Sigma_{B J}$, (d) $Q^{2}$ and $y$. 

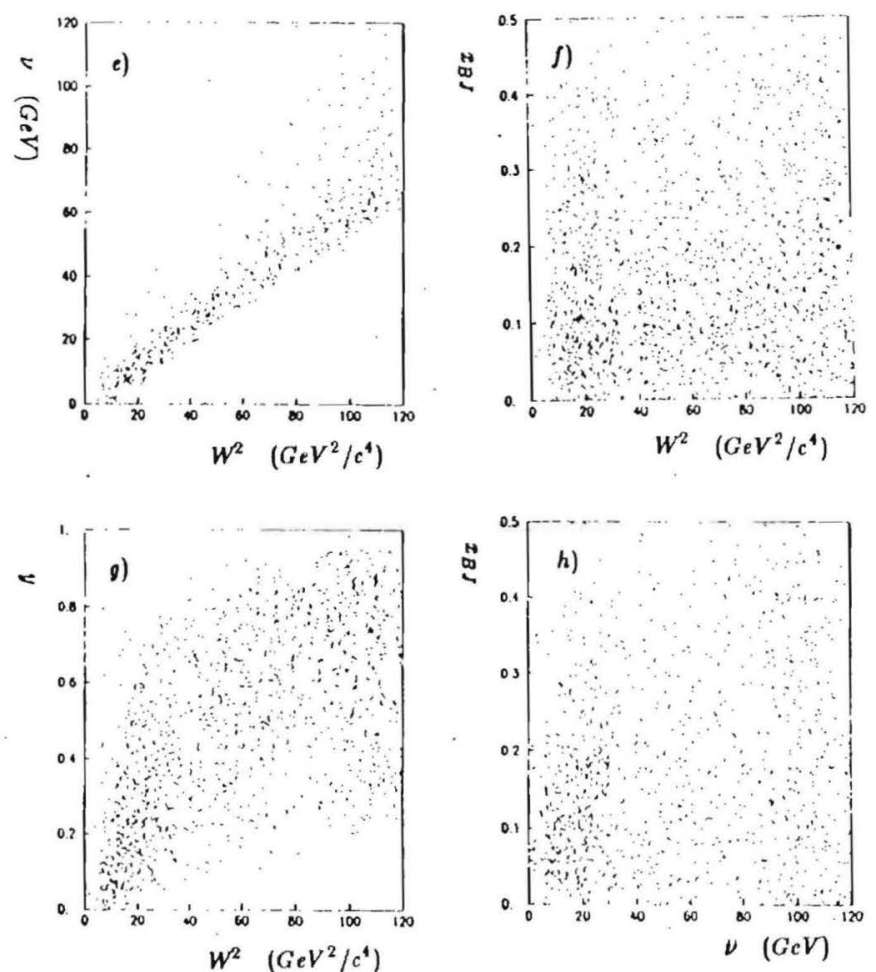

FIGURE 7.2.4 eontinued.

Proportionsl dot plota (see text) ahowing correlations between (c) $W^{2}$ and $\nu$, (n) $W^{2}$ and $x_{B J}$, (g) $W^{2}$ and $y,(\mathrm{~h}) \nu$ and $x_{B J}$.
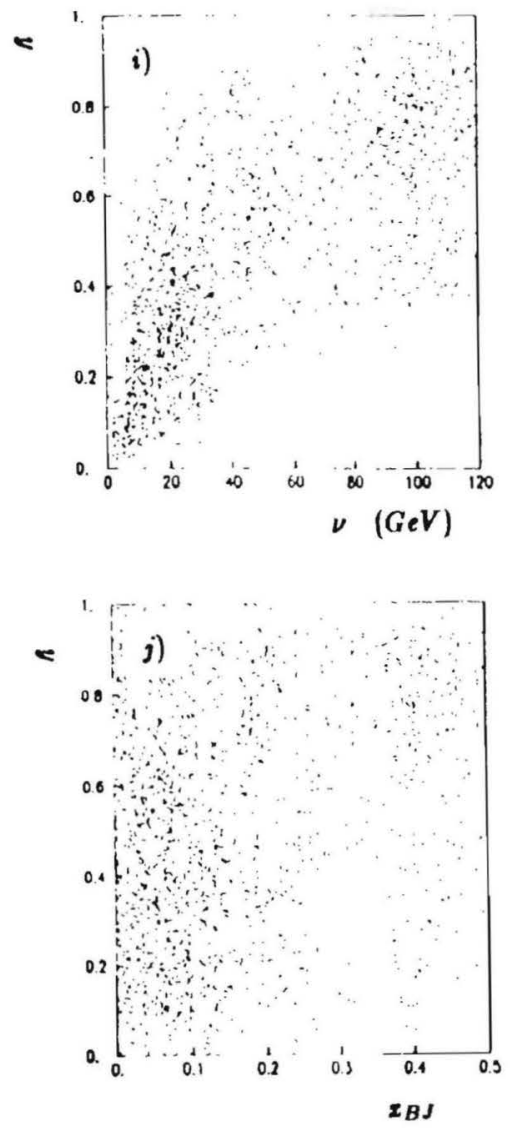

FIGURE 7.8.4 continued.

Proportionsl dot plots (see text) showing correlations between (I) $\nu$ and $y$ and $(\jmath) x_{B} J$ and $y$. 

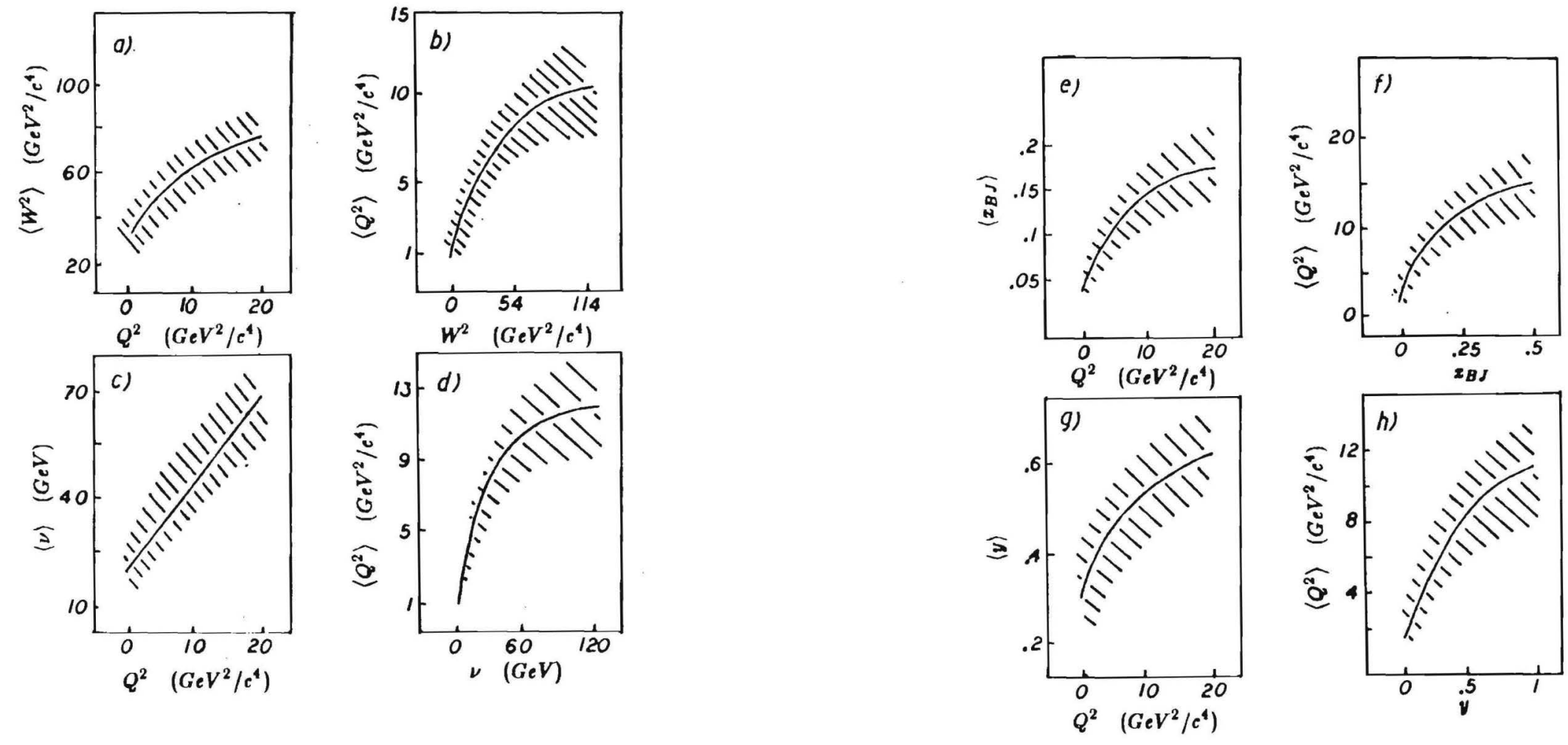

\section{FIGURE 7.1.5.}

Correlation plots ahowing average and dispersion. (a) and (b) $Q^{2}$ and $W^{2}$, (c) and (d) $Q^{2}$ and $\nu$. The solid line in the average, the shaded area shows the dispersion in the average.

FIGURE 7.8.5 continued.

Correlation plots showing average and dispersion for (o) and (f) $Q^{2}$ and $x_{B J},(\mathrm{~g})$ and $(\mathrm{h}) Q^{2}$ and $y$ 

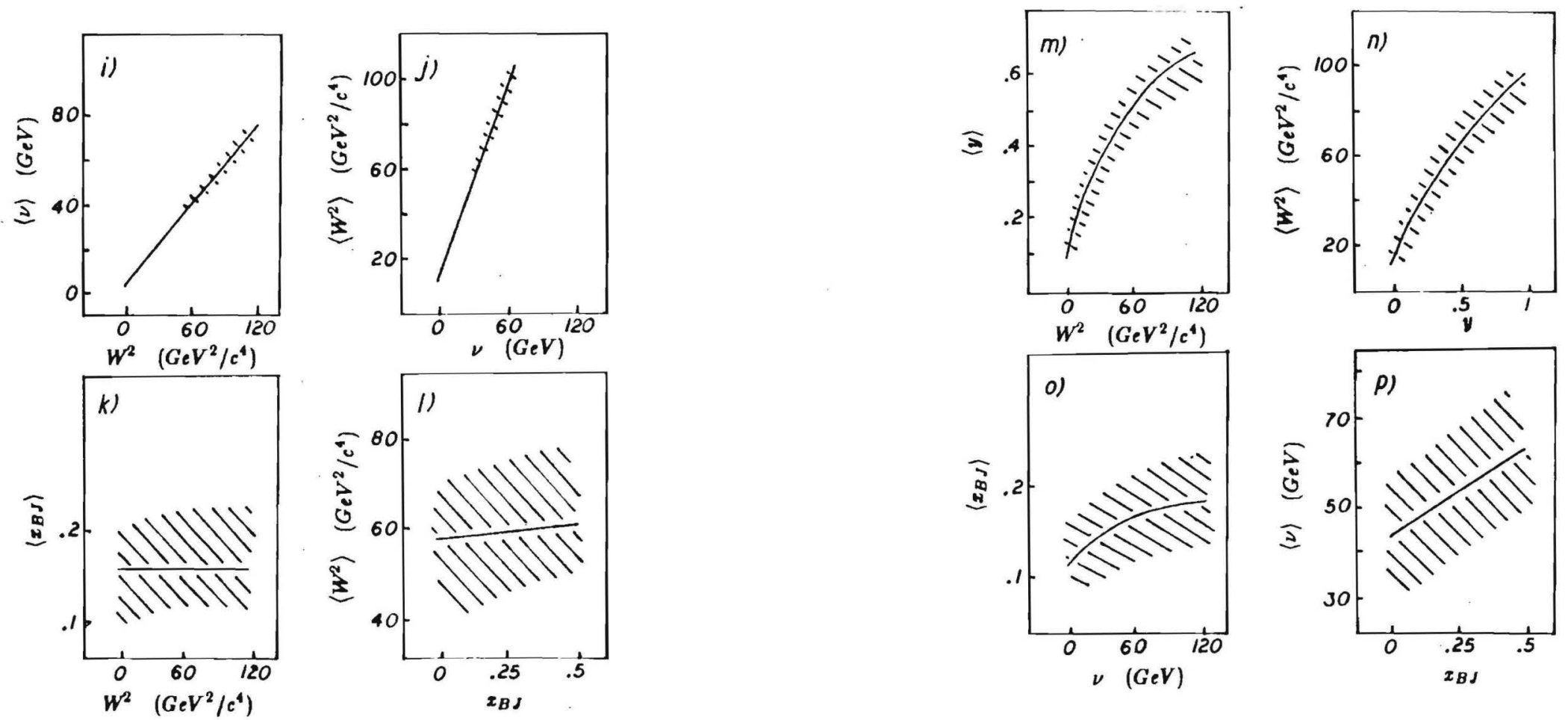

FIGURE 7.8 .5 contlnued.

FIGURE 7.2.5 eontinued.

Correlation plots showing average and dispersion for (1) and (J) $W^{2}$ and $\nu$, (k) and (I) $W^{2}$ and $x_{B}$ J.

Correlation plots showing average and dispersion for (m) and (n) $W^{2}$ and $y,(0)$ and $(\mathbf{p}) \nu$ and $x_{B J}$ 

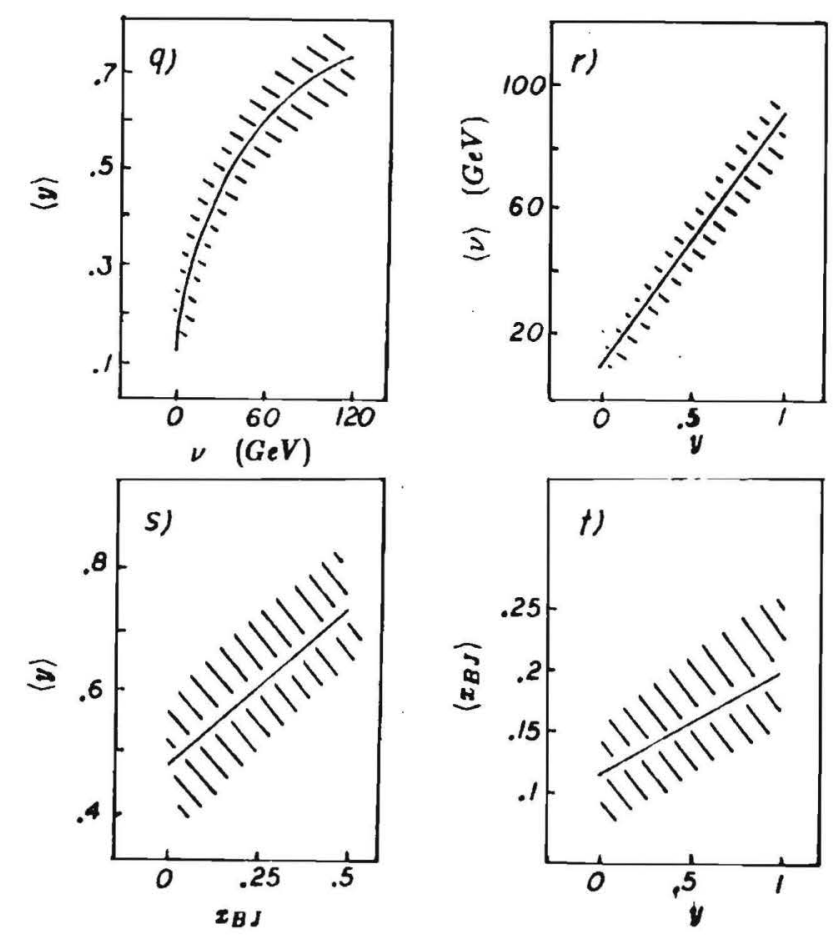

\section{FIGURE 7.8.5 continued.}

Correlation plots showing average and dispersion for $(\mathbf{q})$ and $(\mathbf{p}) \boldsymbol{v}$ and $\boldsymbol{y}$, (s) and $(t) \Sigma_{B J}$ and $y$. enough to calculate the remaining three, excluding the $W^{2}, Q^{2}, \nu$ combination.

Correlations between all possible combinations of $x_{B J}, v, W^{2}, Q^{2}, \nu$ are shown in figures 7.2.4 and 7.2.5 for the $\left.{ }^{(} \bar{\nu}\right)$ Ne data in our experiment. For clarity, these correlations are presented in two ways. Figure 7.2.4 consists of proportional dot plots where the number of dots in any given area on the plot is proportional to the number of events in that area, with seperate event parameters on each axis. Figure 7.2.5 shows the average of one variable plotted as a function of another variable; the shaded area shows the dispersion $D$ in the average - large dispersions indicate poor correlations. The figures are for $\nu \mathrm{Ne}$ only since $\nu \mathrm{Ne}$ has better statistics than $\tilde{\nu} \mathrm{Ne}$. Correlations in $D \mathrm{Ne}$ are similar.

Figures 7.2.4 and 7.2.5 demonstrate that essentially all variables are correlated to some extent in E546, with the exception of $x_{B} J$ and $W^{2}$ (or equivalently $x_{B J}$ and $\nu$ ), which evidently are totally uncorrelated. Particularly striking is the correlation between $W^{2}$ and $\nu$, which are essentially linear functions of each other. $\nu$ and $Q^{2}$ are highly correlated, as are $W^{2}$ and $y$ and $\nu$ and $y \cdot y$ and $x_{B} J$ show a slight correlation but are largely independent.

The linear relationship between $W^{2}$ and $\nu$ leads to an interesting observation. The boost to the hadronic center-of-mass, $\gamma_{c m}$, is given by

$$
\gamma_{e m}=\frac{E_{l a b}}{W}=\frac{\nu+m_{p}}{W}
$$

or in terms of $W^{2}, Q^{2}$

$$
\gamma_{c m}=\frac{W^{2}+Q^{2}+m_{p}^{2}}{2 m_{p} W}
$$


Then the linear relationship between $\nu$ and $W^{2}\langle\nu\rangle \approx 0.85\left\langle W^{2}\right\rangle$ observed in our data implies that

$$
\gamma_{c m} \approx 0.85 W+\frac{m_{p}}{W}
$$

Since $W \gg m_{p}$ for nearly all the data, we have $\left\langle\gamma_{c m}\right) \approx 0.65 W$ or $\left\langle\beta_{c m}\right) \approx$ $\sqrt{1-\left(2.4 / W^{2}\right)}$ for essentially all the E546 data

\section{7.8. Comparison of inclusive distributions in $\left(\bar{\nu}^{\prime}\right.$ Ne and $\pi^{ \pm}$Ne.}

This section compares inclusive distributions $(1 / \sigma) d \sigma / d \theta$ for $\theta=|\overrightarrow{\mathbf{p}}|$, $p_{T}, x_{F}, z_{l a b}, z_{c m}, y_{l a b}$, and $y_{c m}$, between $x^{+}$Ne and $\nu$ Ne and between $\pi-\mathrm{Ne}$ and $\nu \mathrm{Ne}$. The distributions presented here are in $N^{\text {ohower; }}$ equivalent distributions in $\boldsymbol{N}^{-}$have been studied and no significant differences were seen. Therefore the $N^{\text {ohower }}$ distributions are presented due to their increased event statistic

A theorem by Adler ${ }^{[1]}$ has established that in a scattering conflguration he calls the "parallel configuration", $\pi \mathrm{Ne}$ and $\nu \mathrm{Ne} \mathrm{CC}$ scattering give identical results in terms of inclusive distributions. The parallel configuration holds when

\section{$\overline{\mathbf{p}}_{\nu} \| \boldsymbol{B}_{\mu} \quad$ (or equiralently $Q^{2} \approx 0$ ),}

where $\overrightarrow{\mathbf{p}}_{\nu}$ is the incoming momentum of $\left.{ }^{\prime}\right)_{\mu}$ and $\overrightarrow{\mathbf{p}}_{\mu}$ is the outgoing momentum of the $\mu^{ \pm}$. To stay below the order of lepton mass correction effects in the theorem, one should require $Q^{2} \leq m_{r}^{2}$, ie $Q^{2} \leq$ $0.02 \mathrm{GeV}^{2} / \mathrm{c}^{4}$. There is the additional constraint $\nu^{2} / \mathrm{m}_{\mathrm{r}}^{2} \gg 1$, but this is satisfed for all E546 data. The limit $Q^{2} \leq m^{2}$ is, of course, unrealizable in our data.

Given the above constraints, Adler derives for the squared spinaveraged matrix element in $\left(\bar{\nu}^{\prime}\right.$.

$$
\left\langle|\mathcal{M}|^{2}\right\rangle=\frac{4 m_{N}^{2}}{g_{r}^{2}} \frac{k_{10} k_{20}}{\nu^{2}} g_{a}^{2}\left[1-\frac{m_{\mu}^{2}}{2\left(m_{\pi}^{2} k_{20}+m_{\mu}^{2} \nu\right)}\right]^{2}|\mathcal{M}(x+\alpha \rightarrow \beta)|^{2} .
$$

where $g_{a}$ is the axial vector beta-decay coupling constant and $g_{r}$ is the renormalized $x$-nucleon couping constant (for the definition of other quantities in eqtn. 7.3.1, see figure 7.3.1). From eqtn. 7.3.1 it is evident that inclusive distributions for $W^{2}=s$ (weighted to the total cross section) for the produced particles of $\beta$ must be equivalent in $\left(\bar{\nu}^{-} \mathrm{Ne}\right.$ and $x^{ \pm} \mathrm{Ne}$.

The only inputs to this proof other than the standard Dirac formalism for weak interactions are CVC (Conserved Vector Current) and PCAC (Partially Conserved Axial Current), so the proof holds for any V-A interaction. The key feature to note in the proof is that in the double limit $Q^{2} \rightarrow 0$ and $m_{4} \rightarrow 0, k_{1}$ and $k_{2}$ must be null rectors and so $k_{1}$ is proportional to $\boldsymbol{k}_{2}$. Then by symmetry all antisymmetric terms in $\boldsymbol{k}_{1} \boldsymbol{k}_{2}$ drop out of the squared spin-averaged lepton current, leaving only terms $\sim k_{1 \mu} k_{2 \nu}$ in the matrix element. These can be rewritten as proportional to $q_{1}$ ie $q_{\mu} q_{\nu} J_{\mu}^{\text {Had }} J_{\nu}$ Had. Once written in this form (that of a divergence), PCAC immediately lets us extrapolate and establish the theorem.

It is interesting that in this case Adler's use of the old notation ie pre-Standard Model, proves useful and suggestive. It is doubtful that this theorem would ever suggest itself using the standard variables and formalism of the gauge-theory picture of weak interactions (although of 


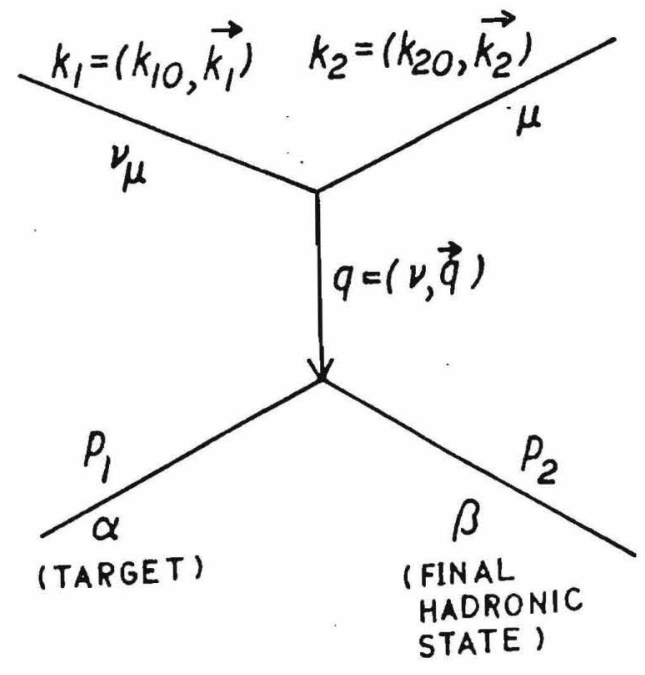

FIGURE 7.8.1.

Deflnition of quantities for the "parallel conffguration" . course the gauge-theory formalism is far more fundamental and revealing of what is actually(?) happening in weak interactions).

Having established that $\pi^{ \pm} \mathrm{Ne}$ and $\left(\bar{\nu}^{\prime}\right) \mathrm{Ne}$ are identical In the $Q^{2} \rightarrow 0$ limit, we look to see where they differ for finite $Q^{2}$. Inclusive distributions were compared for four $Q^{2}$ ranges: $0 \leq Q^{2} \leq 2,2 \leq Q^{2} \leq 10,10 \leq$ $Q^{2} \leq 30$, and $Q^{2} \geq 30$ (where the units are $\mathrm{GeV}^{2}$, and $W^{2}$ is constrained to the $\pi p 30 \mathrm{GeV} / \mathrm{c}$ equivalent band).

All distributions are consistent with the Adler theorem, with the variation between $\pi^{ \pm} \mathrm{Ne}$ and $\left(\bar{\nu}^{)} \mathrm{Ne}\right.$ in all inclusive distributions decreasing as $Q^{2}$ decresses, as expected if the distributions overlap in the limit $Q^{2} \rightarrow 0$. The most significant differences between $\pi^{ \pm} \mathrm{Ne}$ and $\left(\bar{\nu}^{\prime} \mathrm{Ne}\right.$ occur in $(1 / \sigma) d \sigma / d y,(1 / \sigma) d \sigma / d x r$, and $(1 / \sigma) d \sigma / d \varepsilon_{0} m$. These three distributions are given in figures 7.3.2-7.3.5 ( $\left.y_{\mathrm{cm}}\right)$, figures 7.3.0-7.3.9 $(2 \mathrm{r})$, and figures 7.3.10-7.3.13 $\left(\mathrm{s}_{\mathrm{cm}}\right)$ for the four $Q^{2}$ ranges.

We expect that transformations along the rapidity axis from one Lorente frame to another will not alter the shape of the rapidity distribution. The rapidity is the Lorents velocity parsmeter along this axis and so this Lorents transformation corresponds to a global addition of a constant, or a net shift of the distribution. $(1 / \sigma) d \sigma / d y_{\text {las }}$ and $(1 / \sigma) d \sigma / d y_{c m}$ compared between $\pi^{ \pm} \mathrm{Ne}$ and $(\bar{\nu}) \mathrm{Ne}$ do indeed retain their relative form provided we account for the rapidity shift between $\left(\bar{\nu}^{\prime}\right) \mathrm{Ne}$ and $\pi^{ \pm} \mathrm{Ne}$ due to the spacelike mass of the $W$ propogator.

In comparing $y_{i a b}$ distributions, it can be important to note that the neutrino distributions can have a net shift pelative to the $\pi$ distributions. This shift originates from the spacelike mass of the $W$-propogator and changes the boost to the hadronic CM for neutrinos. The $\gamma_{\mathrm{cm}}$ for neutrinos 
has already been calculated in eqtn. 7.2.2. To calculate $\gamma_{c m}$ for $\pi$ incident note that

$$
\gamma_{c m}^{\pi}=\frac{E_{l a b}}{\sqrt{s}}=\frac{E_{\pi}+m_{p}}{\sqrt{s}} .
$$

Ignoring the $\pi$ mass, from $s=(\pi+p)^{2}$ it follows that $E_{\pi}=\left(s-m_{p}^{2}\right) / 2 m_{p}$ and that

$$
\gamma_{c m}^{\pi}=\frac{s+m_{p}^{2}}{2 m_{p} \sqrt{s}} .
$$

Now if two systems have a relative $\beta$, then the rapidity shift between the two systems is $v^{\prime}=-\ln [\gamma(1+\beta)]$, with $\beta$ positive in the direction of increasing rapidity. Then $y_{\nu}^{\prime}-y_{\pi}^{\prime}=\ln \gamma_{c m}^{*} / \gamma_{c m}^{\nu}$, where I take $\beta_{c m}^{\pi} / \beta_{c m}^{\nu} \approx$ 1 , ie considering only relativistic systems, and thus we have

$$
\begin{aligned}
v_{\nu}^{\prime}-v_{\pi}^{\prime} & =\ln \frac{a+m_{p}^{2}}{W^{2}+Q^{2}+m_{p}^{2}} \\
& =-\ln \left(1+\frac{Q^{2}}{W^{2}+m_{p}^{2}}\right)
\end{aligned}
$$

Therefore the $y_{\text {lab }}(\nu)$ distribution is shifted to higher V/ab relative to the $y_{l a b}(x)$ distribution by the absolute value of the amount given in eqtn. 7.3.3.

Whereas this shift may be appreciable in the case of high $Q^{2}$ and low $W^{2}$, for the parallel configuration it is zero. To see how this shift affects the vlab distributions studied here, note that for the $2 \leq Q^{2} \leq 10$ band, the maximum possible shift is given by taking the low edge of the $W^{2}=30 \mathrm{GeV}^{2}$ bin used and the high edge of the $Q^{2}$ bin used:

$$
\begin{aligned}
\Delta y_{\max }\left(Q^{2}<10\right) & =0.22 \\
\Delta y_{\max }\left(Q^{2}<2.5\right) & =0.06
\end{aligned}
$$

and the average shift (taking the centers of the bins) is

$$
\begin{aligned}
\left\langle\Delta_{y}\left(Q^{2}<10\right)\right\rangle & =0.08 \\
\left\langle\Delta y\left(Q^{2}<2.5\right)\right\rangle & =0.03 .
\end{aligned} .
$$

These shifts sre, of course, absent for $y_{\mathrm{cm}}$.

The rapidity shift is still largely negligible for $Q^{2} \leq 10$, but can be significant for $Q^{2}$ greater than 10.

If the $(1 / \sigma) d \sigma / d y_{\text {lab }}$ distribution is compensated on an event-by-event basis for the $Q^{2}$ shift, then $(1 / \sigma) d \sigma / d \nu_{c m}$ and $(1 / \sigma) d \sigma / d y_{\text {lab }}$ compared between $\pi^{ \pm} \mathrm{Ne}$ and $\left(\bar{\nu}^{\prime} \mathrm{Ne}\right.$ are identical except for a net shift, and only $(1 / \sigma) d \sigma / d \nu_{0 m}$ will be presented here. (If no compensation is performed, then using the lab frame fixes the lower edge of the $(1 / \sigma) d \sigma / d y_{1}$ ab distribution at $p_{\boldsymbol{\|}}=\mathbf{0}$ or $\boldsymbol{y}_{\mathrm{m} \text { in }}=\mathbf{0}$ for a stationary target; with a possible backwards fermi momentum in $\mathrm{Ne}$ of $\approx 200 \mathrm{MeV} / \mathrm{c}, V_{\min } \approx-1$. Uncompensated viab distributions do overlap at the lowest rapidities for all $Q^{2}$, with a $y_{\min } \approx-1$.)

For the $y_{o m}$ plots, note how $(1 / \sigma) d \sigma / d y_{\mathrm{cm}}$ in $\nu$ Ne behaves as a function of $Q^{2}$. At the highest $Q^{2}$, the $\nu_{\mathrm{em}}$ plot is symmetric in the forwards and backwards hemispheres; but as $Q^{2}$ decreases an excess of particles developes in the backwards hemisphere, which is associated with target production effects. At the most forward edge of $y_{c m}$, which is associated with projectile production, the distributions are similar or overlap, showing that production associated with the projectile or fast quark is insensitive to $Q^{2}$ effect. The increasing relative particle production in the target hemisphere as $Q^{2}$ decreases evidently indicates greater nuclear contributions at these low $Q^{2}$.

Comparing $(1 / \sigma) d \sigma / d y_{0} m$ between $\pi^{ \pm} \mathrm{Ne}$ and $\left(\bar{\nu}^{\prime} \mathrm{Ne}\right.$, we see that as $Q^{2}$ increases the peak of the distribution in $\left(\bar{\nu}^{\prime} \mathrm{Ne}\right.$ is pushed to lower $\nu_{0}$ and the excess of $\pi^{ \pm} \mathrm{Ne}$ relative to ${ }^{(} \bar{\nu}^{\prime} \mathrm{Ne}$ in the forward hemisphere becomes more pronounced. Even in the $Q^{2}<2$ band, there is a leading particle 
185

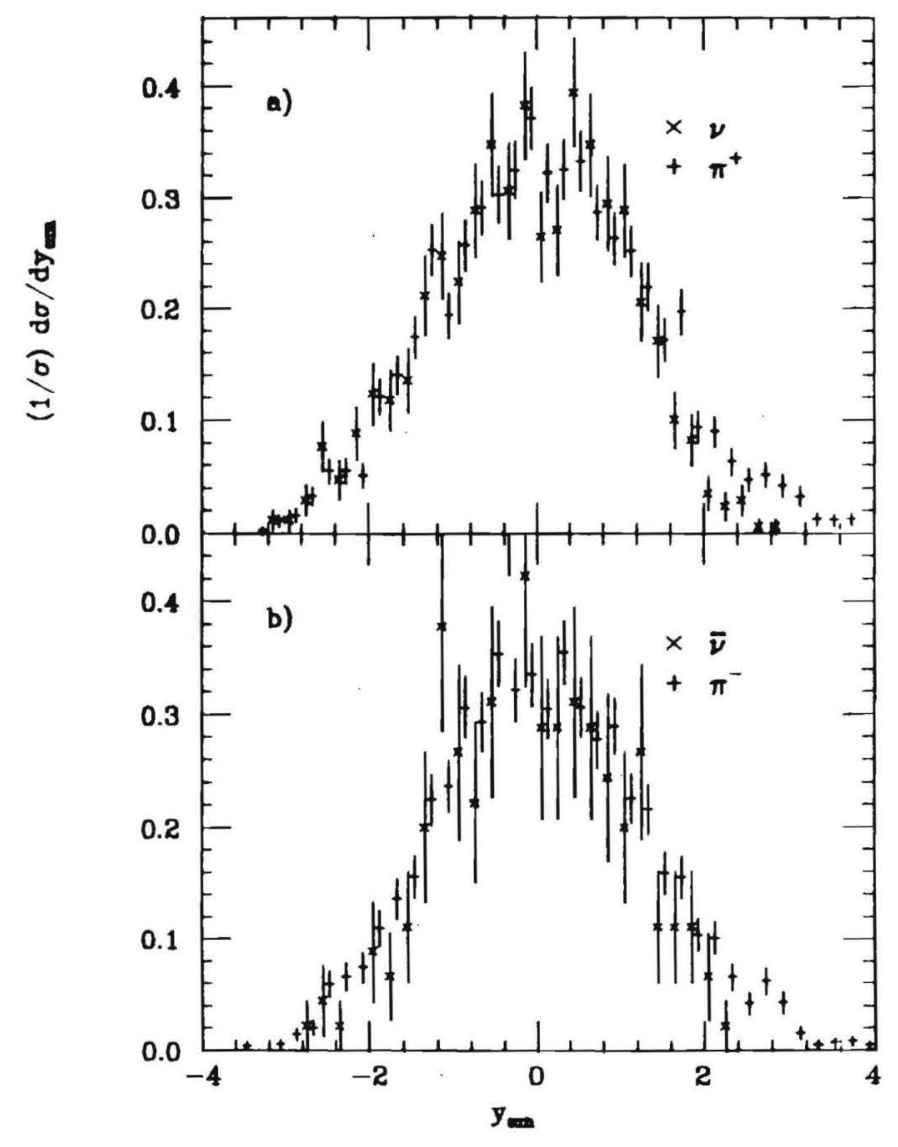

FIGURE 7.8.2.

$(1 / \sigma) d \sigma / d y_{e m}$ for $Q^{2}<2$. (a) $\pi^{+} \mathrm{Ne}$ and $\nu \mathrm{Ne}$ and $(\mathrm{b}) \pi^{-} \mathrm{Ne}$ and $\nu \mathrm{Ne}$ $W^{2}=30 \mathrm{GeV} / \mathrm{c}$ equiv.
186

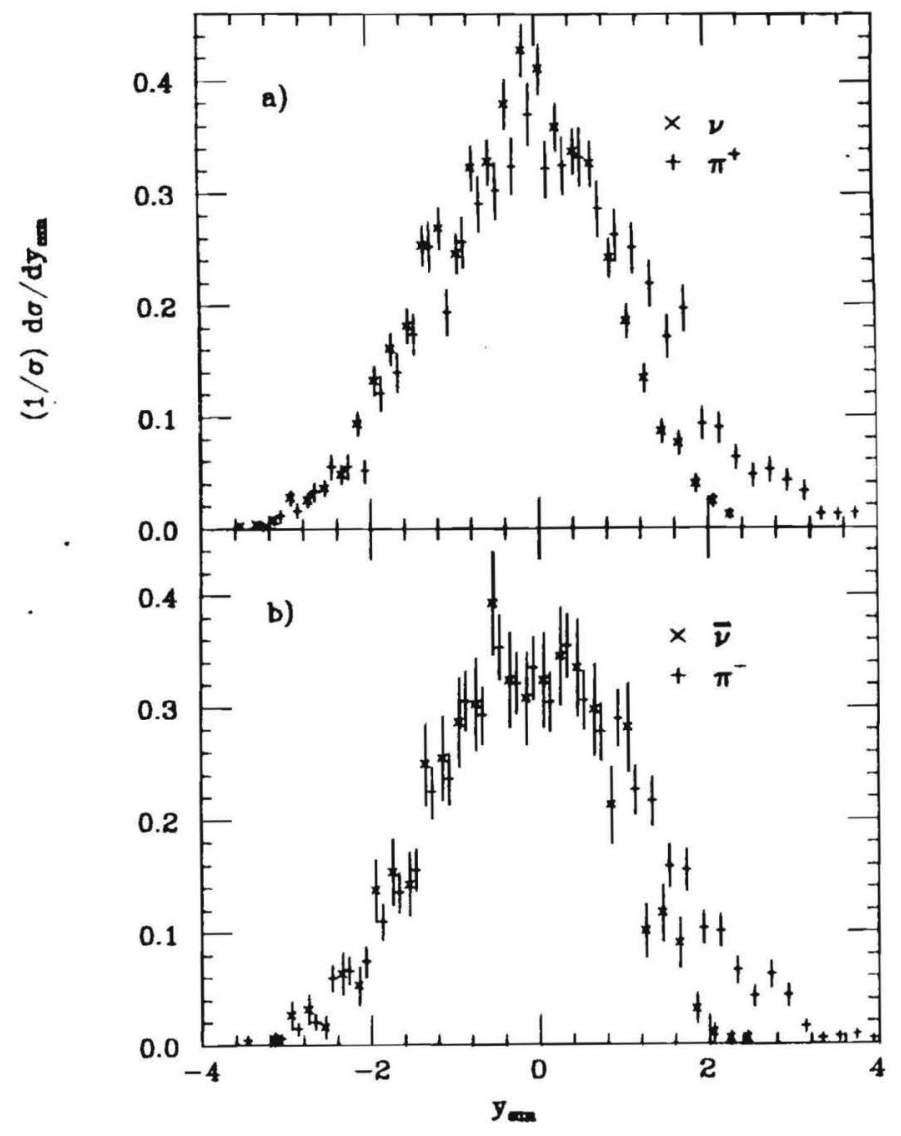

FIGURE 7.8.8.

$(1 / \sigma) d \sigma / d y_{\mathrm{cm}}$ for $2<Q^{2}<10$. (a) $\pi^{+} \mathrm{Ne}$ and $\nu \mathrm{Ne}$ and (b) $\pi^{-\mathrm{Ne}}$ and $\nu \mathrm{Ne} . W^{2}=30 \mathrm{GeV} / \mathrm{c}$ equiv. 


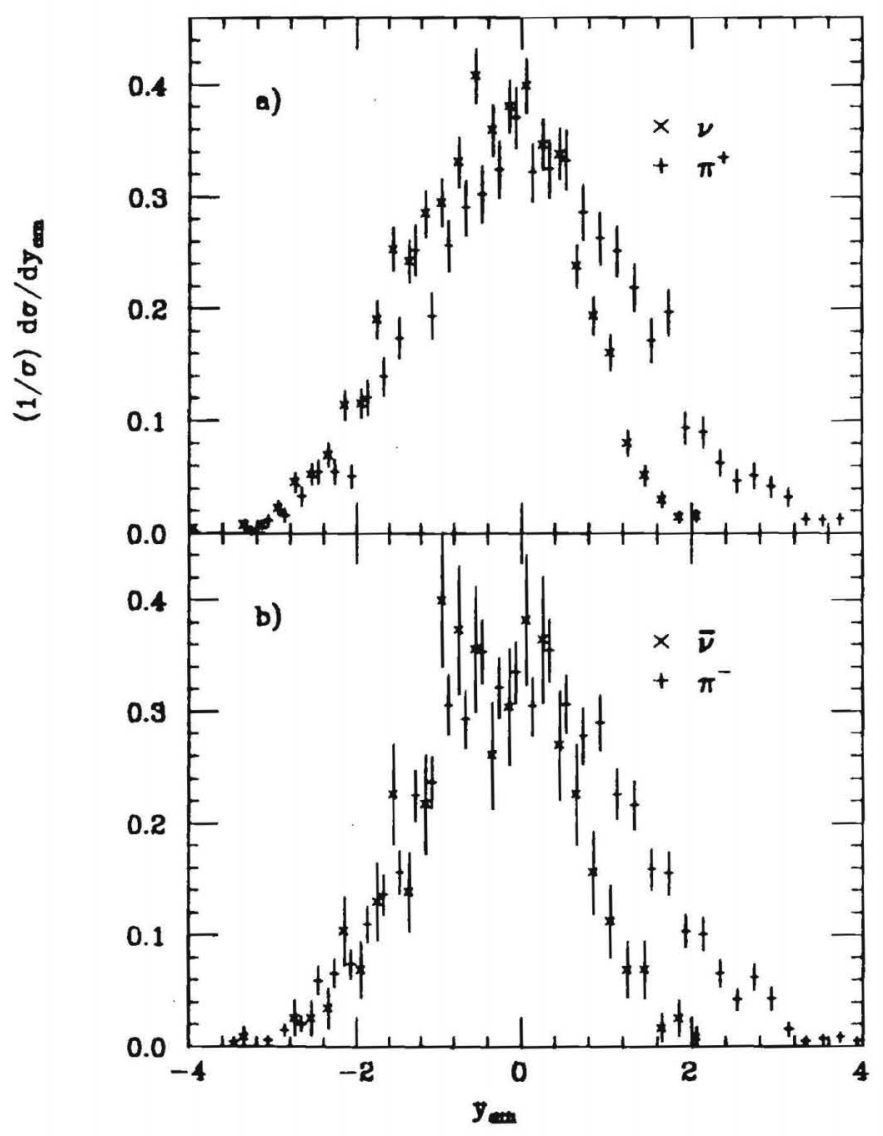

FIGURE 7.8.4.

$(1 / \sigma) d \sigma / d y_{c m}$ for $10<Q^{2}<30$. (a) $\pi^{+} \mathrm{Ne}$ and $\nu \mathrm{Ne}$ and (b) $\pi^{-} \mathrm{Ne}$ and DNe. $W^{2}=30 \mathrm{GeV} / \mathrm{c}$ equir.
188

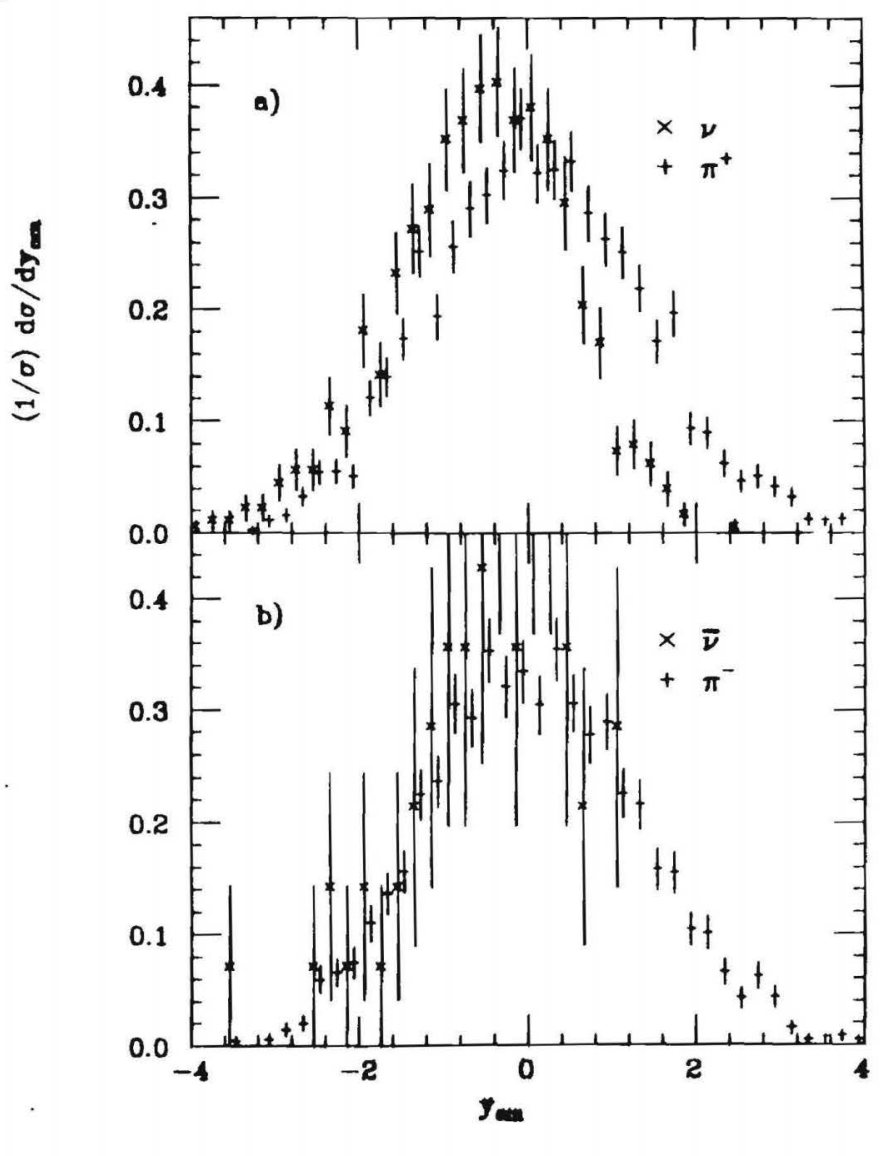

FIGURE 7.8.6.

$(1 / \sigma) d \sigma / d y_{o m}$ for $Q^{2}>30$. (a) $\pi^{+}$Ne and $\nu$ Ne and $(b) \pi^{-}$Ne and $D N e$. $W^{2}=30 \mathrm{GeV} / \mathrm{c}$ equir. 
effect for $y_{c m}>2$ in $\pi^{ \pm} \mathrm{Ne}$ not present in $\left(\bar{\nu}^{-}\right) \mathrm{Ne}$, as expected from the different quark content of the two systems (see section 2.5)

$(1 / \sigma) d \sigma / d z_{c m}$ is equivalent within errors for $\left(\bar{\nu}^{\prime}\right) \mathrm{Ne}$ and $\pi^{ \pm} \mathrm{Ne}$ when $Q^{2}<2$. As $Q^{2}$ increases the ${ }^{(} \bar{\nu}^{\prime}$ Ne distribution loses its symmetry around $z_{c m}=0$ and developes an excess of particles in the backwards hemisphere both relative to the forward hemisphere and relative to $\pi^{ \pm} \mathrm{Ne}$. The distribution is also depleted at the highest $Q^{2}$. The shift to the backwards hemisphere is expected from QCD ocesn scattering

$(1 / \sigma) d \sigma / d x_{F}$ agsin demonstrates the leading particle effect of $\pi^{ \pm} \mathrm{Ne}$ relative to ${ }^{(} \bar{\nu}^{\prime} \mathrm{Ne}$ as $Q^{2}$ increases.

For the distributions not explicitly shown here, $(1 / \sigma) d \sigma / d|\overrightarrow{\mathbf{p}}|$ is equiv. alent within errors for $\pi^{ \pm} \mathrm{Ne}$ and $\left(\bar{\nu}^{-}\right) \mathrm{Ne}$ for all $Q^{2}$ bands. $(1 / \sigma) d \sigma / d z_{\text {lab }}$ is also equivalent for the two systems except for a long tail that develops for $\pi^{ \pm} \mathrm{Ne}$ relative to ${ }^{(} \bar{\nu}^{\prime} \mathrm{Ne}$ as $Q^{2}$ increases.

The $p_{T}$ distributions show that $\pi^{ \pm} \mathrm{Ne}$ has an excess of particles relative to ${ }^{(} \bar{\nu}^{\prime} \mathrm{Ne}$ at the peak of the distribution and below, that is, below about $200 \mathrm{MeV} / \mathrm{c}$. One might attribute this excess to the increased amount of nuclear interactions expected in $\pi^{ \pm} \mathrm{Ne}$ relative to $\left(\bar{\nu}^{\prime} \mathrm{Ne}\right.$. To make a rough estimate of the amount of additional $p_{T}$ expected from nuclear rescattering, consider the nuclear Fermi momentum for $\mathrm{Ne}: \boldsymbol{k}_{F}=1.35 \mathrm{fm}^{-1} \approx$ $270 \mathrm{MeV} / \mathrm{c}$. At each rescatter a particle gains an average additional $p_{T}$ of $\left\langle k_{F \perp}\right\rangle=\int \sin \theta k_{F} d \theta / \int d \theta=2 k_{F} / \pi\left(1\right.$ am assuming that $\left\langle p_{\perp}\right\rangle=0$ from other sources because of the symmetry of the Ne nucleus). Then the $\langle\nu\rangle$ scatters in the nucleus can be considered a random walk in two dimensions away from the original $p_{T}=0$, with a step length of $\left\langle k_{F}\right\rangle$ and for $\langle\nu\rangle$ steps.

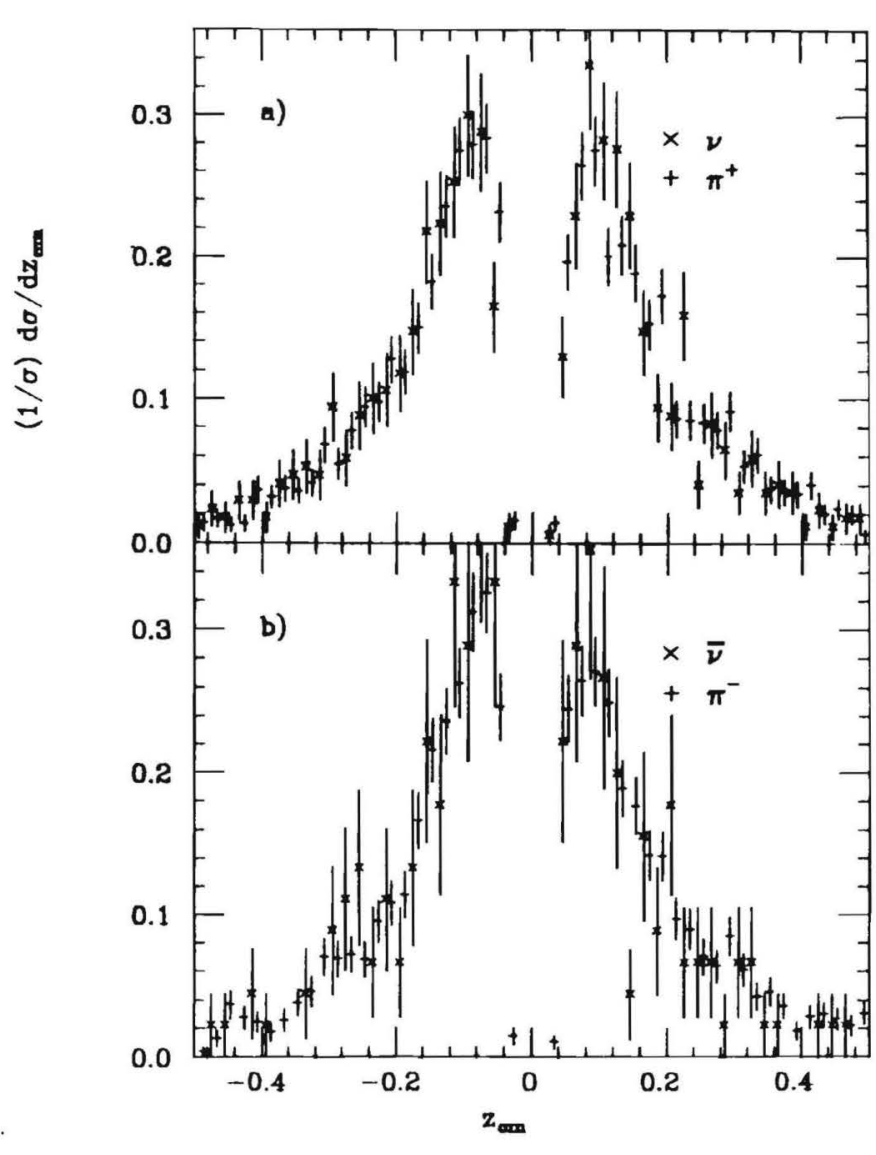

FIGURE 7.8.6.

$(1 / \sigma) d \sigma / d z_{\text {em }}$ for $Q^{2}<2$. (a) $\pi^{+} \mathrm{Ne}$ and $\nu \mathrm{Ne}$ and $(\mathrm{b}) \pi^{-} \mathrm{Ne}$ and $D \mathrm{Ne}$ $W^{2}=30 \mathrm{GeV} / \mathrm{c}$ equir. 
191

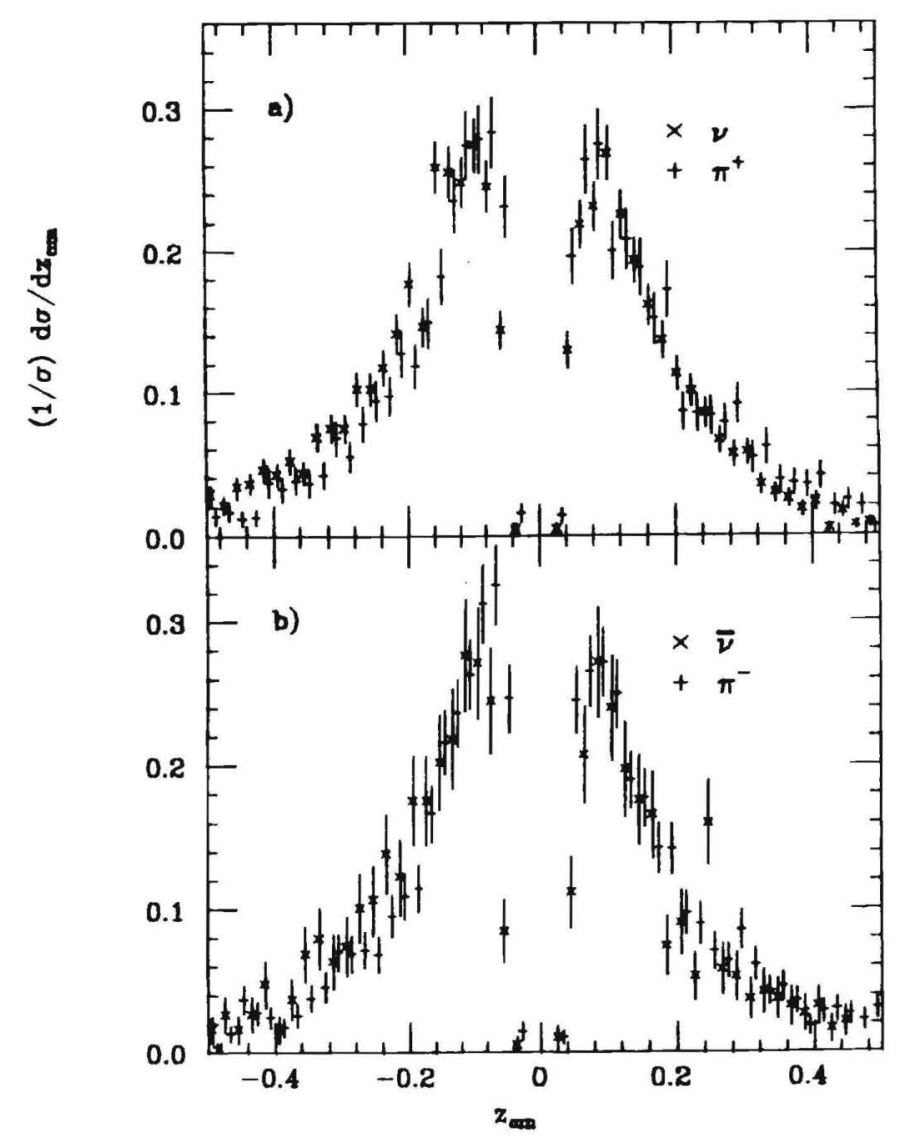

FIGURE 7.8.7.

$(1 / \sigma) d \sigma / d z_{\mathrm{cm}}$ for $\mathrm{z}<Q^{2}<10$. (a) $\pi^{+} \mathrm{Ne}$ and $\nu \mathrm{Ne}$ and (b) $\pi^{-} \mathrm{Ne}$ and DNe. $W^{2}=30 \mathrm{GeV} / \mathrm{c}$ equiv.
182

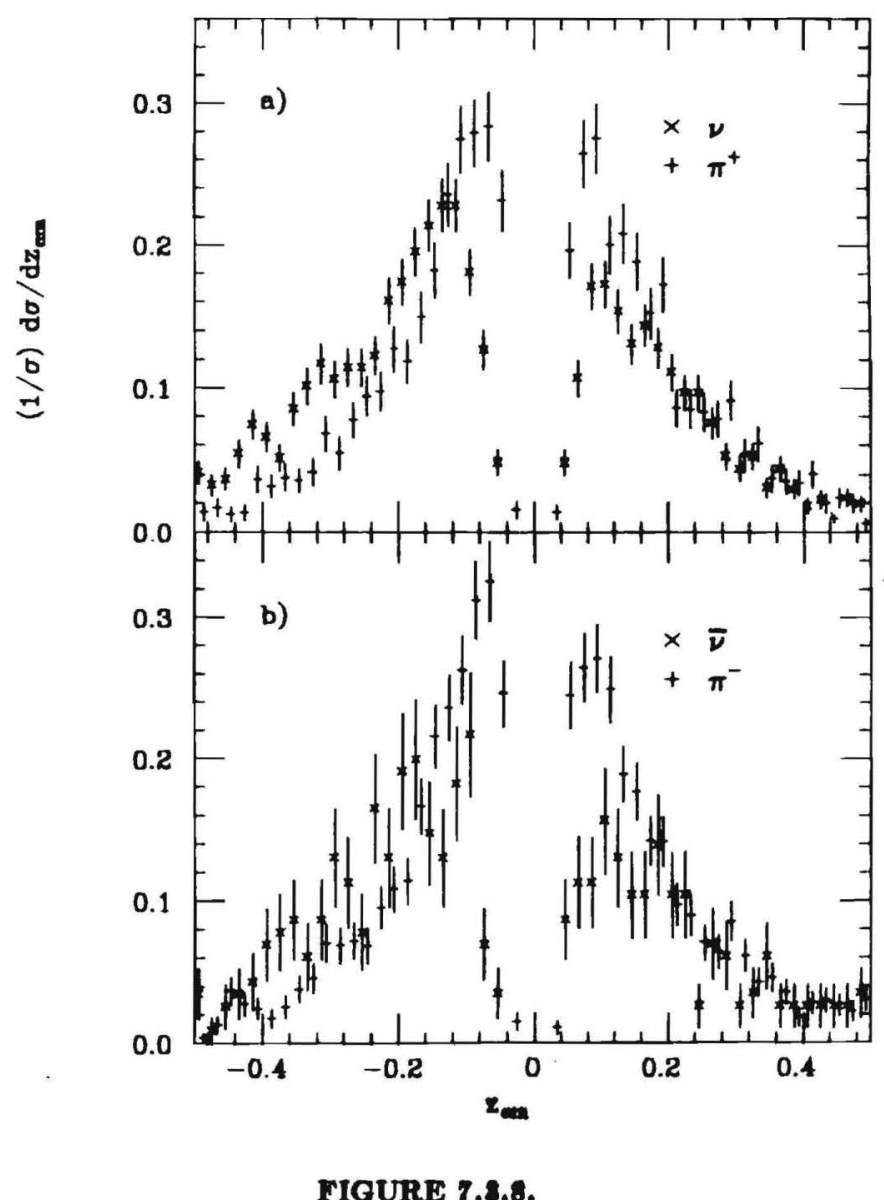

$(1 / \sigma) d \sigma / d z_{c m}$ for $10<Q^{2}<30$. (a) $\pi^{+}$Ne and $\nu$ Ne and (b) $\pi^{-}$Ne and $D N e . W^{2}=30 \mathrm{GeV} / \mathrm{c}$ equir. 


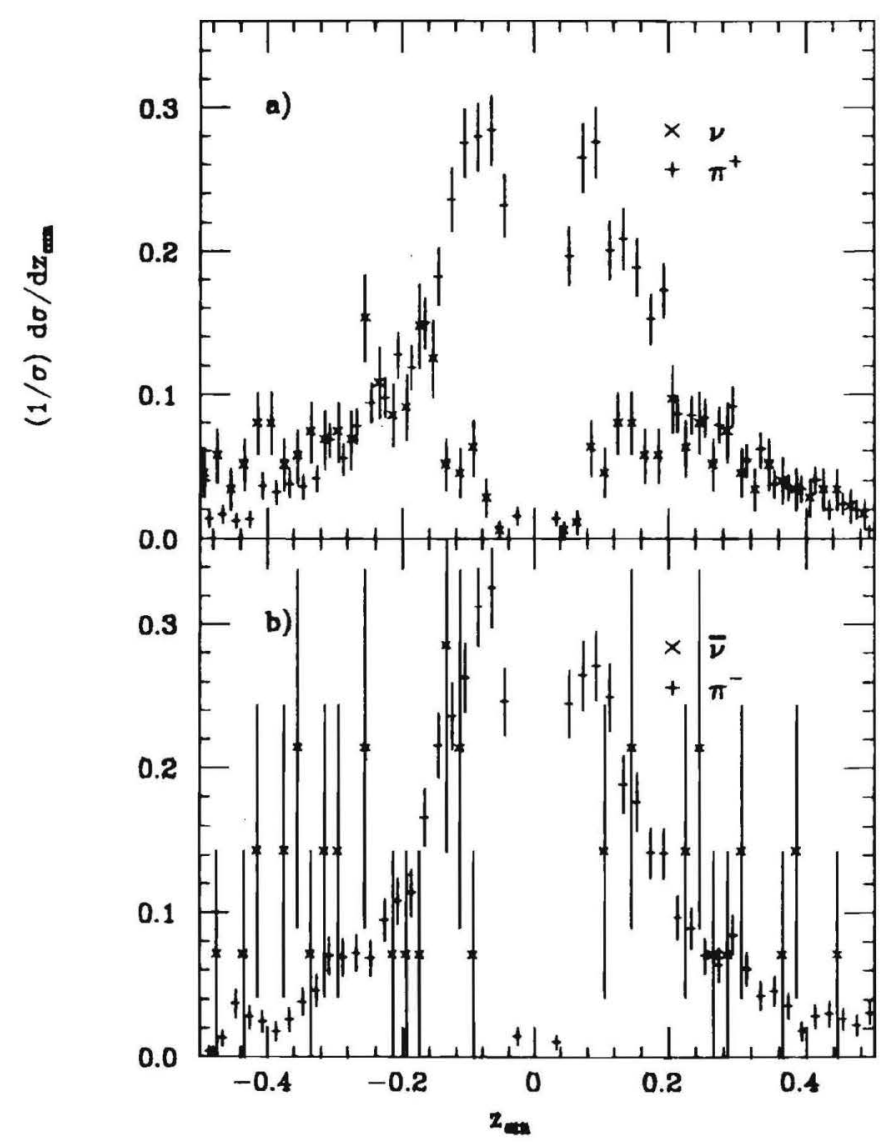

FIGURE 7.8.0.

$(1 / \sigma) d \sigma / d s_{o m}$ for $Q^{2}>30$. (a) $x^{+} \mathrm{Ne}$ and $\nu \mathrm{Ne}$ and $(\mathrm{b}) x^{-} \mathrm{Ne}$ and $\nu \mathrm{Ne}$. $W^{2}=30 \mathrm{GeV} / \mathrm{c}$ equiv.

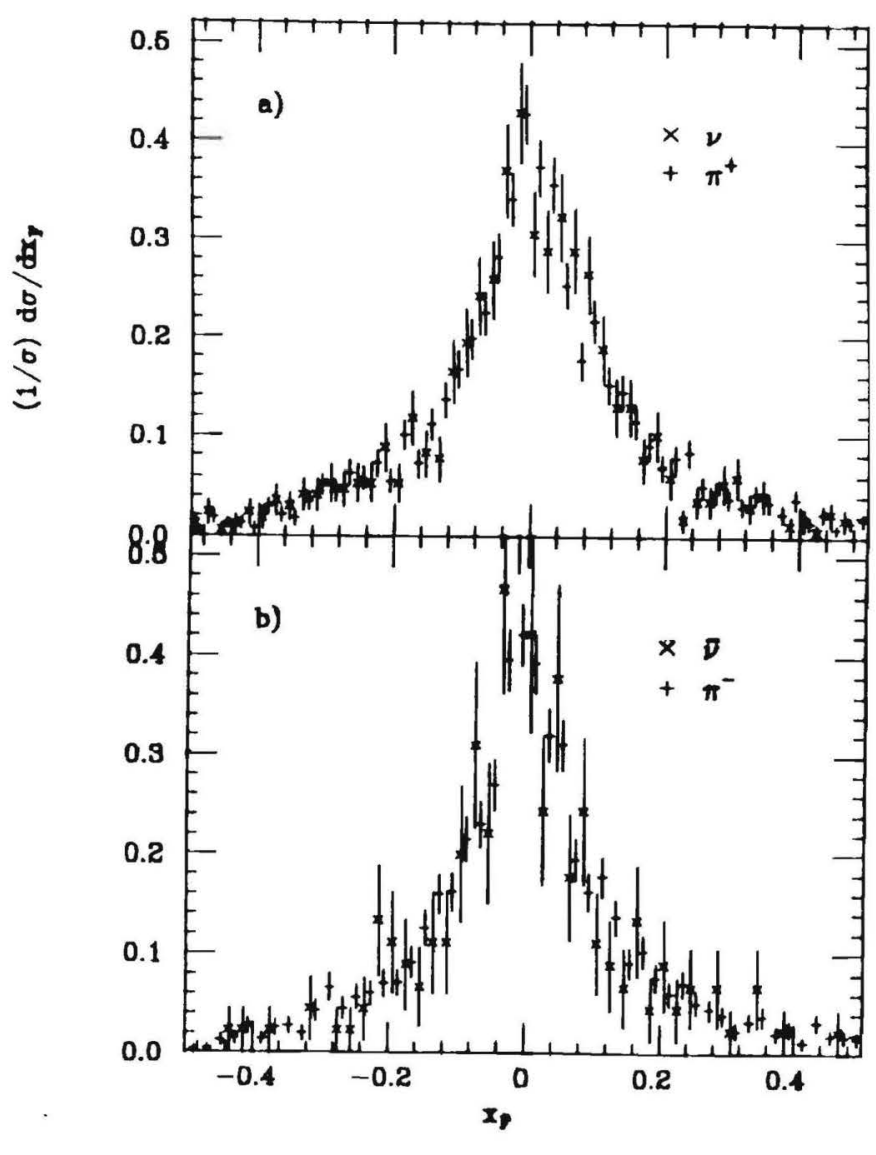

FIGURE 7.8.10.

$(1 / \sigma) d \sigma / d x$ for $Q^{2}<2$. (a) $x^{+} \mathrm{Ne}$ and $\nu \mathrm{Ne}$ and (b) $x^{-} \mathrm{Ne}$ and $D \mathrm{Ne}$ $W^{2}=30 \mathrm{GeV} / \mathrm{c}$ equiv. 


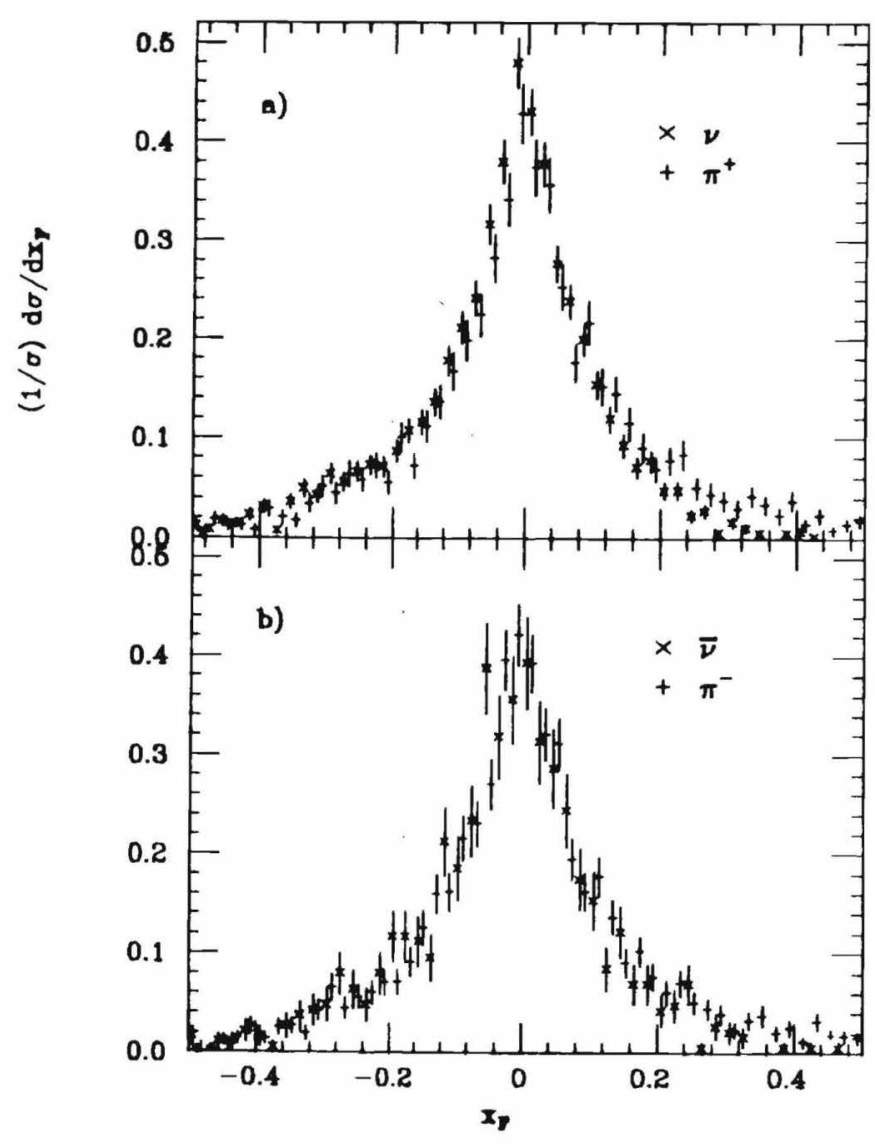

FIGURE 7.8.11.

$(1 / \sigma) d \sigma / d x_{F}$ for $2<Q^{2}<10$. (a) $\pi^{+} \mathrm{Ne}$ and $\nu \mathrm{Ne}$ and (b) $x^{-} \mathrm{Ne}$ and DNe. $W^{2}=30 \mathrm{GeV} / \mathrm{c}$ equiv.

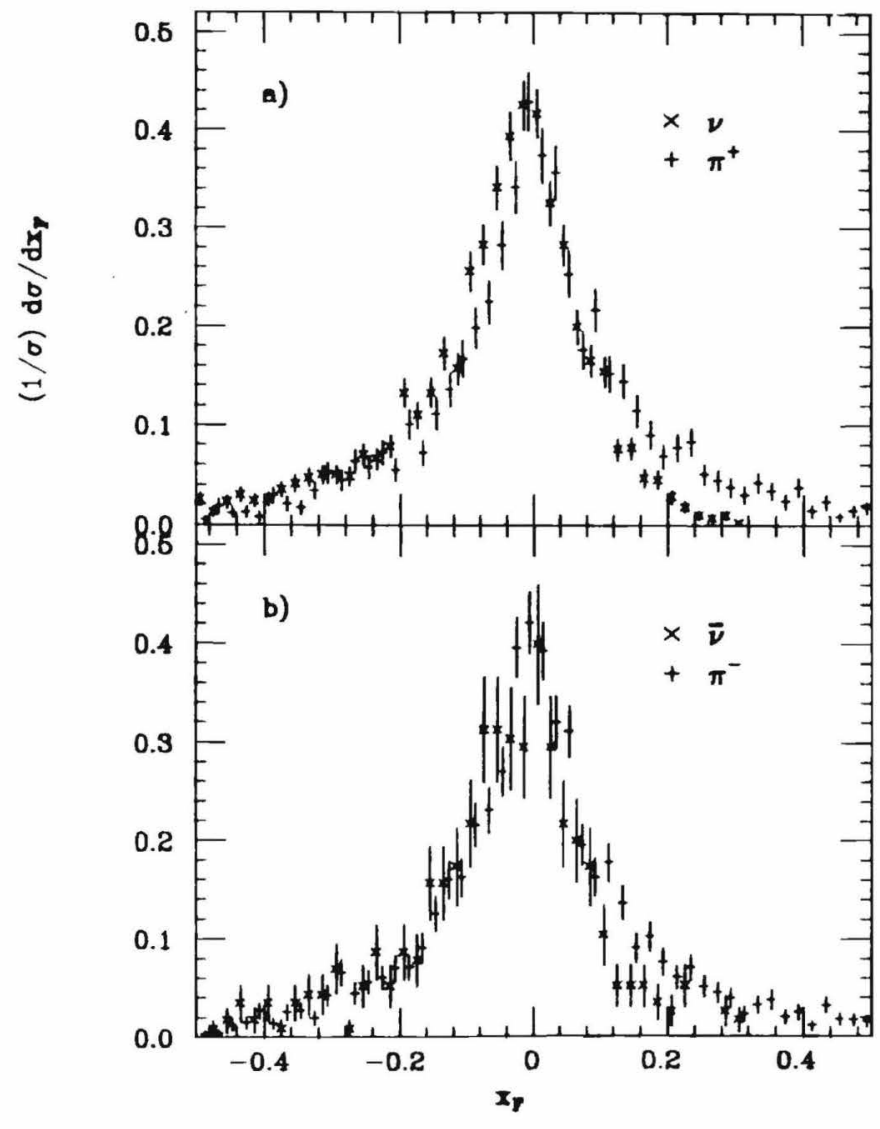

FIGURE 7.8.1\%.

$(1 / \sigma) d \sigma / d x_{F}$ for $10<Q^{2}<30$. (a) $x^{+} \mathrm{Ne}$ and $\nu \mathrm{Ne}$ and (b) $\pi^{-} \mathrm{Ne}$ and $D$ Ne. $W^{2}=30 \mathrm{GeV} / \mathrm{c}$ equiv. 


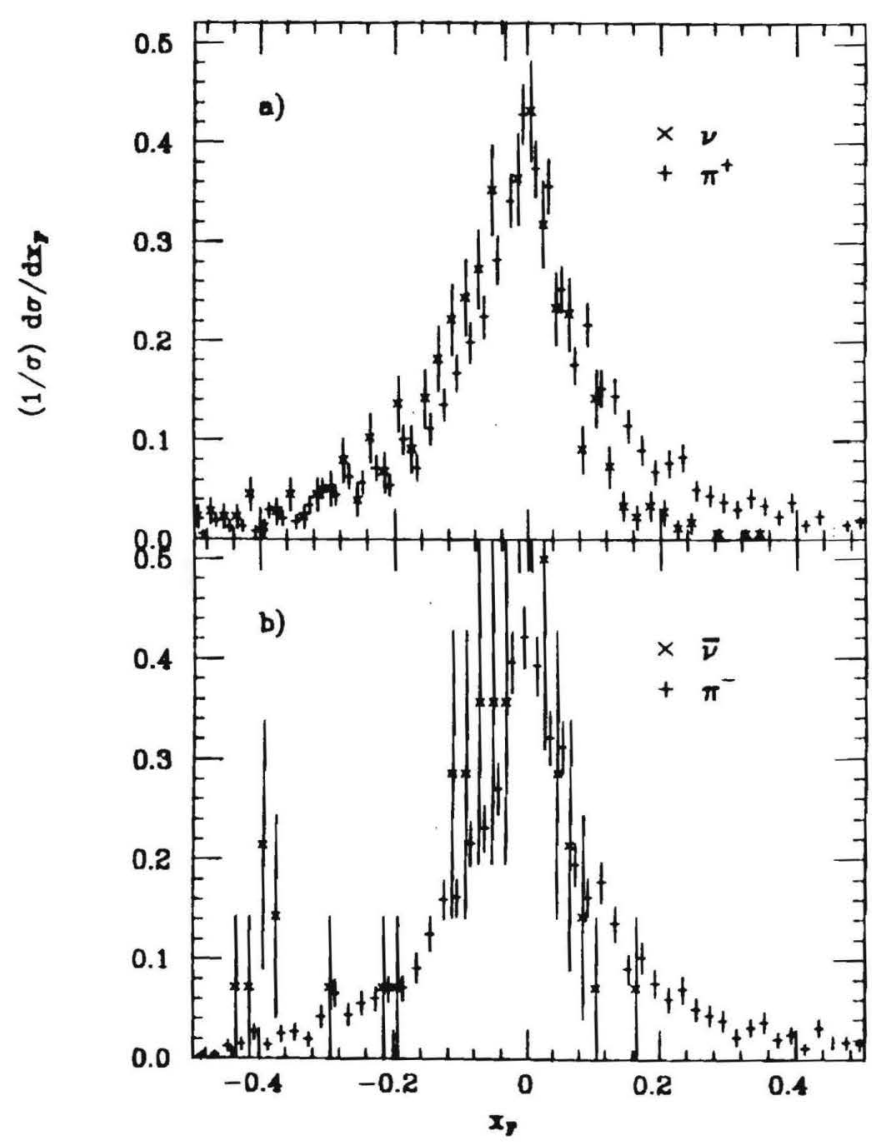

FIGURE 7.8.18.

$(1 / \sigma) d \sigma / d x F$ for $Q^{2}>30$. (a) $\pi^{+} \mathrm{Ne}$ and $\nu \mathrm{Ne}$ and (b) $\pi^{-} \mathrm{Ne}$ and $D \mathrm{Ne}$ $W^{2}=30 \mathrm{GeV} / \mathrm{c}$ equiv.
The mean distance after $n$ steps in a random walk with step length $L$ is $\left\langle\overrightarrow{\mathbf{R}}_{n}^{2}\right\rangle=n L^{2}$. Thus in Ne we have

$$
\begin{aligned}
\sqrt{\left\langle p_{\perp}^{2}\right\rangle_{\text {reseatter }}} & =\sqrt{\nu} \frac{2 k_{F}}{\pi} \\
& \approx \sqrt{\nu} \quad 170 \mathrm{MeV} / \mathrm{c} .
\end{aligned}
$$

If we assume that $\langle\nu\rangle \sim$ pathlength, then the excess in $\pi^{ \pm} \mathrm{Ne}$ compared to $\left(\bar{\nu}^{\prime}\right) \mathrm{Ne}$ will be $O(\sqrt{2})$ times the rescatter $p_{T}$, or about $200-250 \mathrm{MeV} / \mathrm{c}$. Assuming $10-20 \%$ of events involve nuclear rescatters, this effect is $\sim$ 20-50 MeV/c, probably too small to be detected in this experiment. Therefore it is unlikely that any $p_{T}$ difference seen in this experiment is due to nuclear effects.

All these effects for inclusive distributions are consistent with the leading particle effect in $\pi^{ \pm} \mathrm{Ne}$, whose counterpart in $\left(\bar{\nu}^{\prime}\right) \mathrm{Ne}$ is suppressed at high $Q^{2}$.

\subsection{Nuelear particle produetion a a funetion of rapidity.}

Morrison ${ }^{[s]}$ has previously studied $R_{A}(y)$ in $D N e$, that is, the ratio of the number of negative tracks in Ne to the number of negative tracks in hydrogen as a function of rapidity. His definition of $R_{A}(y)$ was

$$
R_{A}(y)=\left[\frac{\left\langle N^{-}\right\rangle_{D N e}}{\left\langle N^{-}\right\rangle_{D p}}\right]_{y}
$$

(The averages are evaluated in $\mathrm{N}^{-}$to avoid problems with misidentified protons.) His data came from CERN experiments WA21 (Dp) and WA59 $(\bar{\nu} \mathrm{Ne})$. He also divided his data into a number of narrow slices in $\nu$, the 0 -component of the virtual W's four-momentum. 


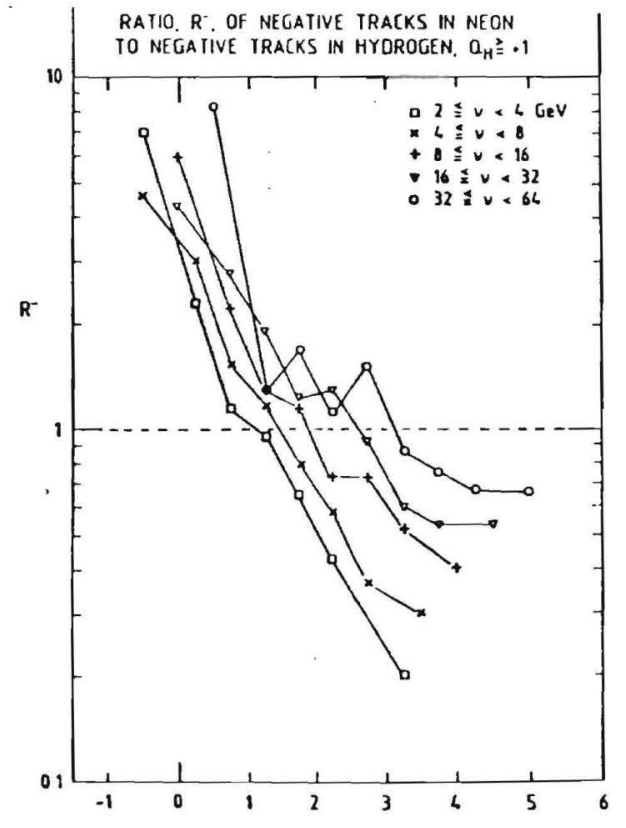

LAB. RAPIOIYY, YLAS

FIGURE 7.4.1.

Morrison results for $R_{A}^{-}$as a function of Vlab and $\nu$ in $D$ Ne. $R_{A}^{-}$uses $\left\langle N^{-}\right\rangle$.
His results are shown in figure 7.4.1 for the variable viab. To summarize, his results indicate that there is a depletion of particles at high $y_{l a b}$ and a strong enhancement at low ylab. Although the amount of enhancement at low $y_{l a b}$ seems to be roughly constant for different $\nu$ slices, the amount of depletion at high $y_{\text {lab }}$ is a strong function of $\nu$. The data for low $\nu$ shows a large amount of depletion while the data for high $\nu$ shows a much smaller amount of depletion. Evidently as $\nu$ increases by a factor of 16 , the value of $R_{A}(y)$ increases by approximately a factor of 4 at the highest vlab.

A similar analysis of our data has been performed. Several important improvements can be made using the $\mathbf{E 5 4 6}$ data; the analysis can be extended to $\nu \mathrm{Ne}$ (where our experiment has good statistical accuracy) in addition to $\nu \mathrm{Ne}$; and much larger values of $\nu$ are accessible.

The NUCTST algorithm has been used to divide our $(\bar{\nu}) \mathrm{Ne}$ data samples into "nuclear" $($ NUCTST $=+2)$ and "nonnuclear" $($ NUCTST $=$ 2) categories. Using these categories, we define $R_{\text {nuc }}(v)$ in a manner similar to the Morrison $R_{A}(v) .\left\langle N^{-}\right\rangle$is used to eliminate misidentifled protons, and $y_{\text {lab }}$ is used. Let $\Delta y$ be a given rapidity interval. If $N_{N U C-+2}^{-}(\Delta y)$ is the number of negative tracks in rapidity interval $\Delta_{\boldsymbol{V}}$ in the NUCTST $=$ +2 category, $N_{N U C-+2}^{T}$ is the total number of events in the NUCTST $=+2$ category, and equivalent symbols are adopted for the NUCTST $=$ -2 category, then

$$
R_{n u c}(\Delta y) \equiv \frac{N_{N U C-+2}^{-}(\Delta y) / N_{N U C-+2}^{T}}{N_{N}^{-} U C-2},
$$

or equivalently

$$
R_{n u c}(y)=\left[\begin{array}{l}
\left(N^{-}\right\rangle_{N U C-+2} \\
\left\langle N^{-}\right\rangle_{N U C--2}
\end{array}\right],
$$


Using $R_{\text {nec }}(y)$ eliminates any normalization or systematic differences between experiments. As shown in sect. 7.2, there are no significant differences between the nuclear and nonnuclear categories in terms of $W^{2}, Q^{2}$, or $\nu$ distributions, except possibly at low $W^{2}$. An important difference between $R_{A}(y)$ as used by Morrison and $R_{n u c}(y)$ used in analyzing our neutrino data is that the net valence charge in the hadronic system after a $\bar{p}$ interaction is $Q^{e f f}=0$, while for an averaged nuclear interaction $\nu(p+n)$ it is $Q^{e f f}=-1 / 3$. Thus there is a net negative charge balance in $R_{A}(y)$. There is no such charge difference in $R_{n u c}(y)$, ie $R_{n u c}(y)$ is effectively normalized to deuterium. Thus as $y \rightarrow \infty$ we might expect $R_{n u c}(y) \rightarrow 1$.

The results for $\boldsymbol{R}_{n u c}(v)$, divided into various $\nu$ slices for neutrino Neon and antineutrino Neon, are presented in figure 7.4.2.

Our results are very similar to the CERN results. The $\nu \mathrm{Ne}$ and $\nu \mathrm{Ne}$ data are very similar, although there is perhaps some tendency for the $\nu$ Ne data to be slightly above than the $\nu$ Ne data at large $y_{\text {lab }}$ and large $\nu$. It is clear from the figures that $\boldsymbol{R}_{\text {nuc }}(\boldsymbol{y})$ is approaching an asymptotic value of 1.0 as $\nu \rightarrow \infty$ and $y_{\text {lab }} \rightarrow \infty$.

The same qualitative features are observed for $Q^{2}$ and $W^{2}$ slices, as shown in figures 7.4.3 and 7.4.4. In $Q^{2}$ there is evidently a slight rise above $R_{\text {nuc }}(y)=1$ for $y_{\text {iab }}>3$ before trending to 1 at the very highest $y_{\text {lab }} . W^{2}$ and $\nu$ are expected to be the same because of the tight correlation shown in section 7.2. $Q^{2}$ and $\nu$ are also correlated, but much more weakly.

The overall results have a simple interpretation. One expects a depletion at large $y_{l a b}$ because some of these large $y_{l a b}$ tracks reinteract in the Ne nucleus, losing rapidity in the process. Since there is no source to

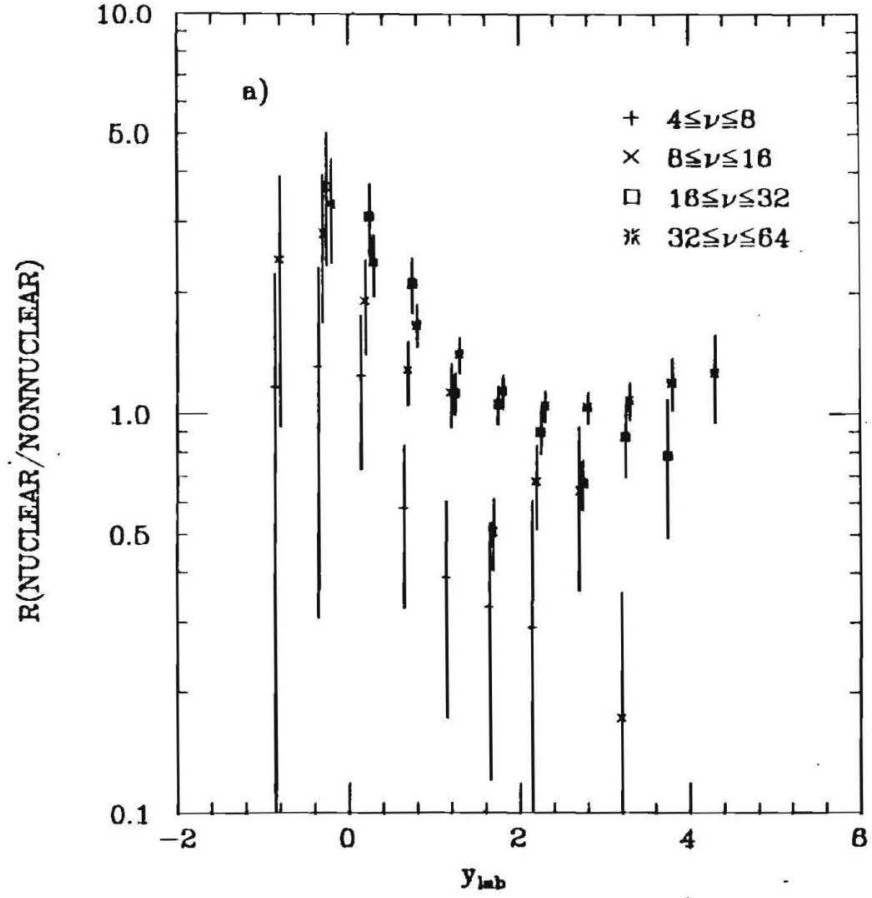

FIGURE 7.4.1.

E546 results for $R_{n \text { nod }}(y)$ as a function of ylab and $\nu$. (a) $\nu \mathrm{Ne}$. 


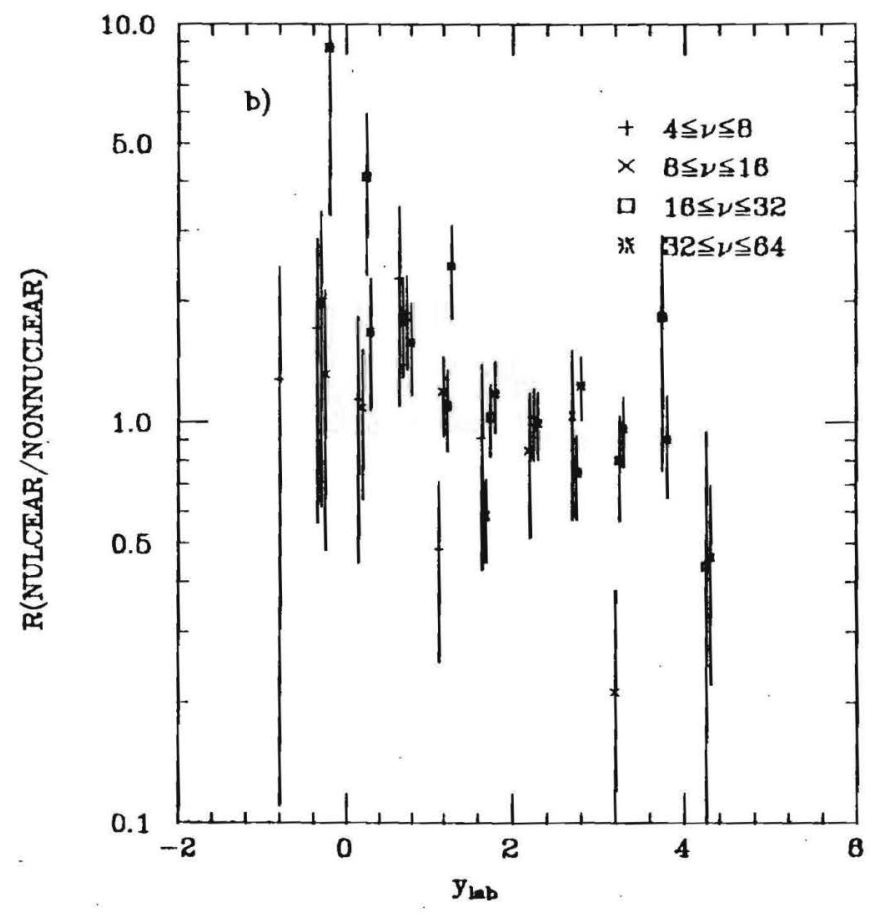

FIGURE 7.4. continued.

E546 results for $R_{\text {neo }}(y)$ as a function of yiab and $\nu$ in (b) $D \mathrm{Ne}$

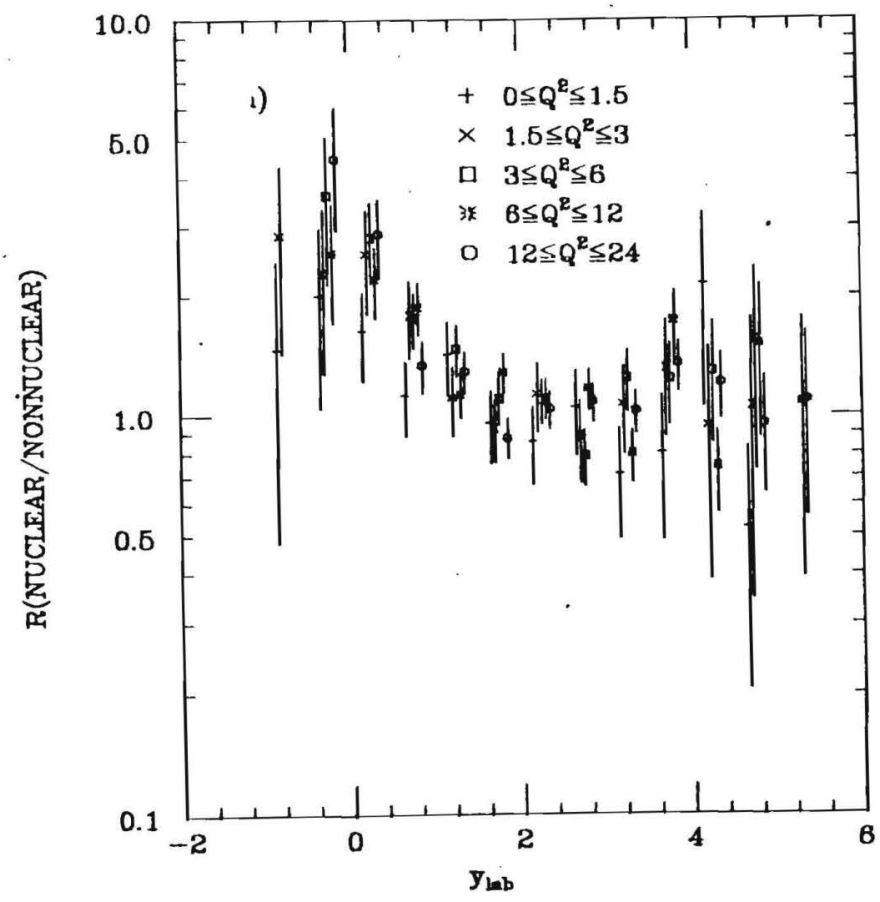

MGURE 7.4.2.

E546 results for $R_{\text {nuo }}(y)$ as a function of ylab and $Q^{2}$. (a) $\nu \mathrm{Ne}$ 


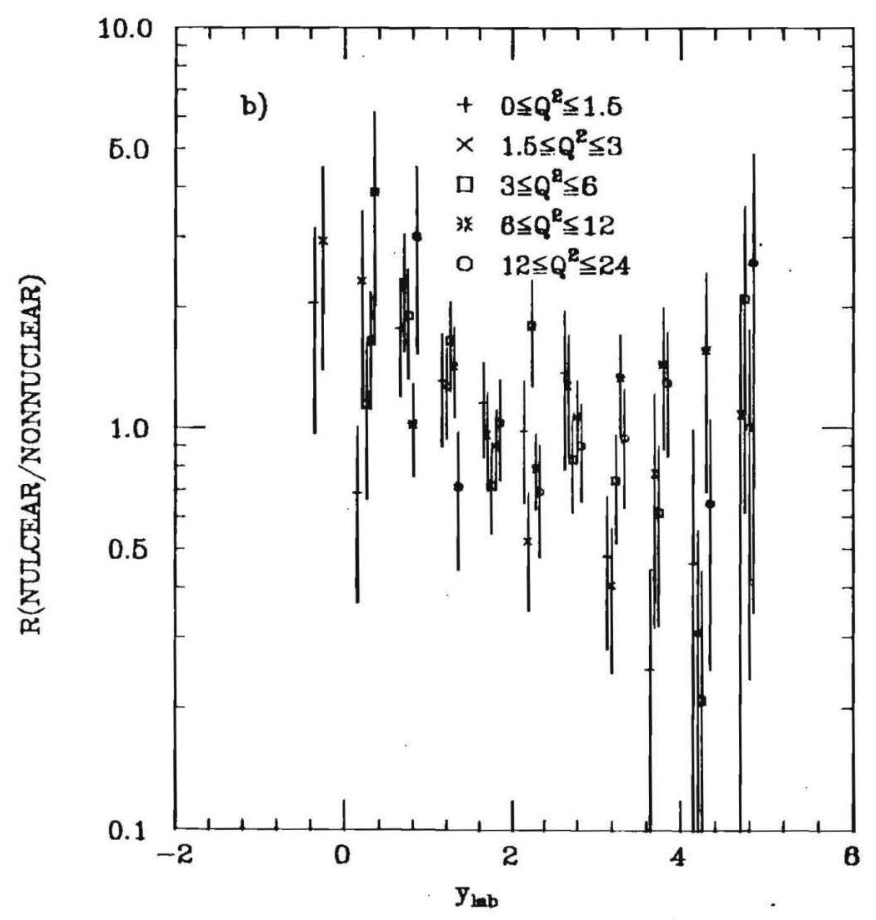

FIGURE 7.4.8 contlaued.

E540 results for $R_{\text {nuo }}(v)$ as a function of vieb and $Q^{2}$ in (b) DNe.

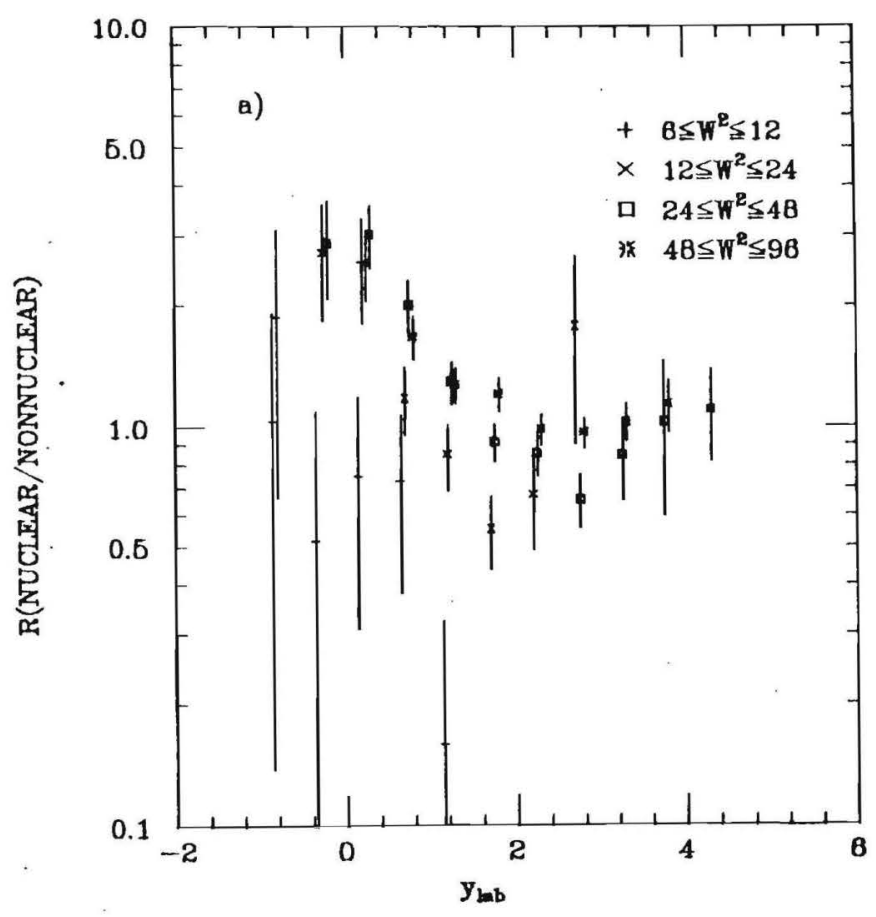

FIGURE 7.4.4.

E546 results for $R_{\text {nuo }}(y)$ as a function of $y_{\text {lab }}$ and $W^{2}$. (a) $\nu$ Ne. 


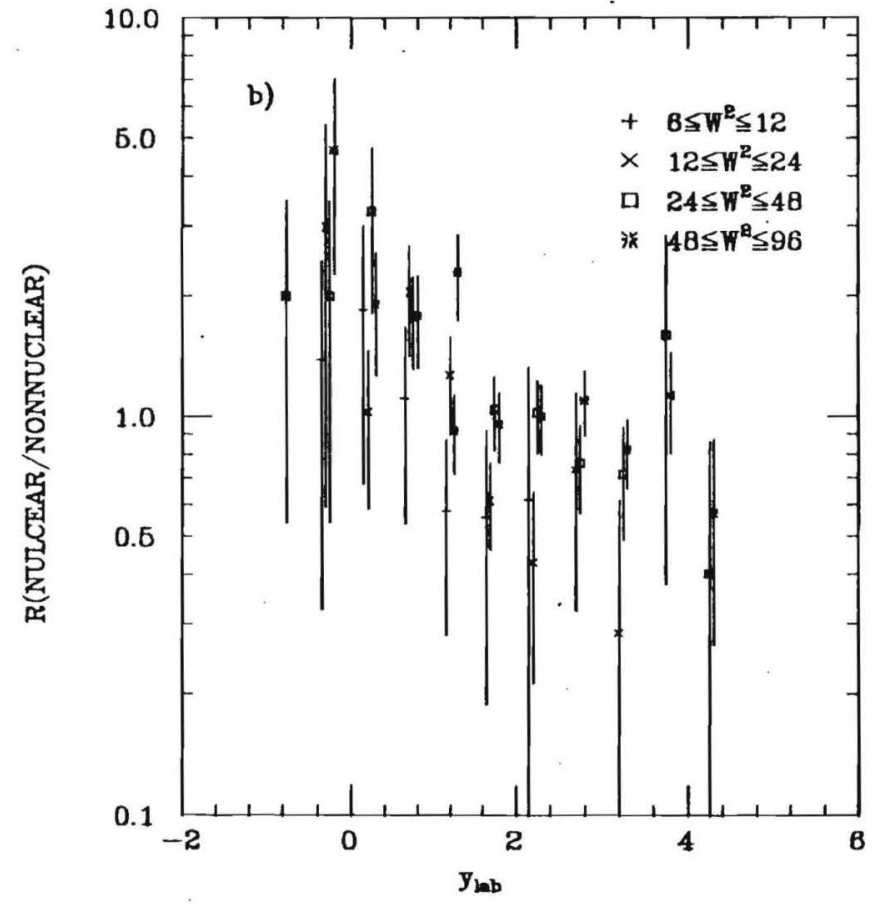

FIGURE 7.4.4 continued.

E540 results for $R_{n u o}(y)$ as a function of $y_{l a b}$ and $W^{2}$ in (b) $D N e$ replenish the highest $y_{\text {lab }}$ tracks, they must be depleted relative to $\left.{ }^{-}\right)^{\prime} p$. Conversely, the rescattered particles and any produced secondaries at a rescatter contribute to lower rapidities, causing an excess of particles at small ylab.

String models or formation-time models predict the observed energytransfer $(\nu)$ dependence: a high energy string will be a "long" string in momentum phase space. If the string fragments to $q \bar{q}$ pairs at a roughly constant interval in momentum space, then the long high energy string will produce most $q \bar{q}$ pairs far from the target and such pairs will not reinteract, so the highest momentum produced mesons will not be strongly depleted. Conversely a "short" low energy string will produce its highest momentum pairs close to the nucleus and there will be more re-interactions. Similarly in formation-time models high energies imply long formation times and few re-interactions; short times and increased re-interactions result from low energy interactions.

\section{f 7.5. Study of Past proton $\left(p_{\text {faot }}\right)$ produetion.}

As discussed in Section 6.3, there are approximately 0.6-0.7 fast protons ( $p_{\text {faot }}$ ) produced per event (a $p_{\text {fat }}$ proton has $|\overrightarrow{\mathbf{p}}| \geq 1.0 \mathrm{GeV} / \mathrm{c}$ and so cannot be identified by ionisation). Some of these protons originate at the primary vertex in the nucleus and some originate at rescatters. This relatively large number of $p_{\text {faot }}$ protons per event is difficult to explain ụsing current models. To gain more information on the production mechanism and properties of $p_{f a s}$ protons, the isospin subtraction technique has been used to obtain their rapidity distribution. 
Since $\mathrm{Ne}$ is an isospin 0 nucleus, the norm of the amplitude for any distribution in $\nu$ Ne should be the same as the norm of the isospin-rotated amplitude in $\boldsymbol{D N e}$. A similar equality must hold for $\pi^{+} \mathrm{Ne}$ compared to $\pi^{-}$Ne. That is,

$$
\begin{aligned}
\left|\Psi^{\boldsymbol{r}^{+}, \nu}\left(p, n, \pi^{+}, \pi^{-}, \pi^{0}, K^{+}, \overline{K^{0}}, \ldots\right)\right| & = \\
& \left|\Psi^{\pi^{-}, \bar{\nu}}\left(n, p, \pi^{-}, \pi^{+}, \pi^{0}, K^{0}, K^{-}, \ldots\right)\right|,
\end{aligned}
$$

where we understand that $\pi^{+}$is to be compared to $\pi^{-}$and $\nu$ to $\bar{\nu}$, but not $\pi^{+}$to $\nu$ or other combinations. Consider the difference distribution in $\theta$,

$$
\Delta \Psi(\theta)=\left|\Psi^{\pi^{+}, \nu}(\theta, \alpha)\right|-\left|\Psi^{\pi^{-}, D}(\theta, \alpha)\right|,
$$

where $\theta$ is any kinematic variable and all other kinematic variables $\alpha$ are held constant. Relating the difference distribution to the cross section we define

$$
\Delta \Phi(1)=\left.\frac{1}{\sigma} \frac{d \sigma^{+}}{d \theta}\right|_{\pi^{+}, \nu}-\left.\frac{1}{\sigma} \frac{d \sigma^{-}}{d \theta}\right|_{\sigma^{-}, \nu}
$$

(the meaning of the argument to $\Delta \Phi$ will become clear later). The isospin invariance expressed in eqtn. 7.5.1 guarantees that the $\pi^{+}$and $\pi^{-}$contributions to $\Delta \Phi$ cancel and only proton production and any difference between $K^{+}$and $K^{-}$production can contribute to $\Delta \Phi$. Any contribution from $K \&$ will be negligible since they are $<5 \%$ of all minimum-ionizing tracks. Also, although $K^{+}$is not the isospin partner of $K^{-}$, we still expect $N^{\nu}\left(K^{+}\right) \approx N^{D}\left(K^{-}\right)$(studies with the LUND monte-carlo verify this assertion).

By using this isospin subtraction technique we can obtain distributions for $p_{\text {faot }}$ in a kinematic variable $\theta$ for both the purely hadronic $\pi^{ \pm} \mathrm{Ne}$ system and the weak $\left(\bar{\nu}^{)} \mathrm{Ne}\right.$ system, and compare the two systems. Note that if $p_{\text {fast }}$ production is mainly associated with rescattering in the nucleus, then we expect $\pi^{ \pm} \mathrm{Ne}$ and $\left(\bar{\nu}^{\prime}\right) \mathrm{Ne}$ distributions to be similar or the same; if $p_{\text {fast }}$ production is intimately associated with the production vertex, then the two systems may vary. Also note that if isospin invariance holds, we can use the alternative form of the isospin difference to obtain

$$
\Delta \Phi(2)=\left.\frac{1}{\sigma} \frac{d \sigma^{+}}{d \theta}\right|_{\sigma^{-}, D}-\left.\frac{1}{\sigma} \frac{d \sigma^{-}}{d \theta}\right|_{\sigma^{+}, \nu} .
$$

(Note that the difference between $\Delta \Phi(1)$ and $\Delta \Phi(2)$ is that the incident particle is exchanged with incident antiparticle, and vice versa. In the $\pi^{ \pm} \mathrm{Ne}$ system, there is nothing in the interaction that couples to flavor, and we expect $\Delta \Phi(1)$ and $\Delta \Phi(2)$ to yield similar amounts of $p_{\text {fast }}$ production. In the ${ }^{\left(\bar{\nu}^{\prime}\right)} \mathrm{Ne}$ system there is an explicit flavor dependence and $\Delta \Phi(1)$ and $\Delta \Phi(2)$ may differ.)

In calculating the isospin subtraction distribution for $(\bar{\nu})$, events were required to have a minimum of two minimum-ionizing tracks because of large uncertainties in the Bonn energy-correction technique for fewer tracks. All identifled protons with $p \leq 1.0 \mathrm{GeV} / \mathrm{c}$ were excluded from the distributions. Tracks were required to have a relative momentum error $\delta p / p<0.31$ (This relative momentum error is placed at a convenient shoulder in the data and accepts $91 \%$ of all minimum ionizing tracks. There are no systematic differences introduced between $\nu$ and $\nu$ by this cut). Similar cuts were applied to $\pi^{ \pm} \mathrm{Ne}$. Also in $\pi^{ \pm} \mathrm{Ne}$, because the fuli multiplicity scan determines the unbiased topological distribution of $\pi^{ \pm} \dot{\mathrm{Ne}}$ at $30 \mathrm{GeV} / \mathrm{c}$, events in the measurement sample were given weights such that their relative multiplicity density in $N^{\text {ohower }}$ was the same as 
that in the full multiplicity scan, and such that the sum of all weighted events yields the same number of events as in the measurement sample (for a discussion, see Chapter 5 ).

The mass given to all tracks in calculating $\Delta \Phi$ was the proton mass $m_{p}$. This amounts to a fixed transformation of $\theta$ which is equiralent by isospin symmetry for $\nu\left(\pi^{+}\right)$and $D\left(\pi^{-}\right)$, so all non-proton charge tracks still cancel in the subtraction and we are left with the correct $p_{\text {fast }}$ distribution. Note that the isospin subtraction technique does not detect protons from baryon-antibaryon production, such as observed by the $E M C$, because such $6 \bar{b}$ pairs cancel in the subtraction. The number of such pairs is in any case small due to baryon mass effects; the LUND montecarlo (see below) predicts that the $\overline{b b}$ contribution to baryon production in our experiment is negligible.

For the $p_{\pi}=30 \mathrm{GeV} / \mathrm{c}$ equiralent sample, there were 1879 neutrino events, 362 antineutrino events, $595 \pi^{+}$events, and $583 \pi^{-}$events. The relative error distributions $\delta p / p$ between $\left(\bar{\nu}^{)} \mathrm{Ne}\right.$ and $\pi^{ \pm} \mathrm{Ne}$ are approximately the same.

Figure 7.5.1(a) presents the $y_{\text {lab }}$ distribution of $p_{\text {faot }}$ calculated using definition 7.5.2 $(\Delta \Phi(1))$ and figure 7.5.1(b) uses the alternative definition 7.5.3 $(\Delta \Phi(2))$. Distributions are shown for both $\pi \mathrm{Ne}$ and $\nu \mathrm{Ne}$. The $p_{\text {fast }}$ ylab distributions are remarkably similar for the two scattering systems, the only discernable difference being that $\Delta \Phi$ evidently extends to lower $y_{\text {iab }}$ in neutrino interactions than in $\pi$ interactions by about $\frac{1}{2}$ a unit of $y_{l a b}$ (in $\Delta \Phi(1)$ ). This difference may only be a result of different scanning and measuring efficiencies for E546 and WA51: Due to increased attention to protons in WA51 scanning and measuring, protons near the border of
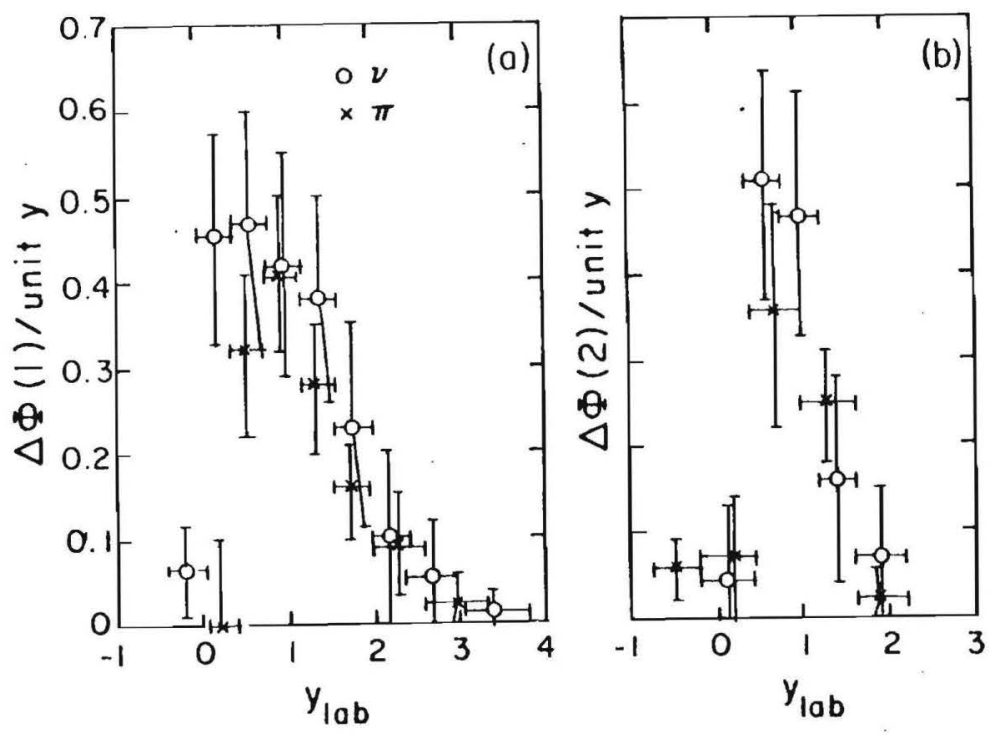

FIGURE 7.6.1.

The fast proton y/ab distribution. Calculated using (a) $\Delta \Phi(1)$ and (b) $\Delta \Phi(2)$. (See text) 
identiflability $(p \approx 1 \mathrm{GeV} / \mathrm{c}$ ) are much more likely to be called "identified" protons in $\pi^{ \pm} \mathrm{Ne}$ (and thus removed from the $p_{\text {fast }}$ plot) than in $(\bar{\nu}) \mathrm{Ne}$. The difference does occur in the region of the rapidity plot where identifled protons occur.

The large difference in the identified proton scan/measure efficiency between WA51 and E546 is clear from figure 7.5.2, the inclusive y/ab distribution for identified protons. Although the efficiency for proton identification is considerably greater for WA51 than E546, the two distributions have qualitatively the same shape: they peak at $y_{l a b} \approx 0$ and drop symmetrically to zero at $\left|y_{l a b}\right| \approx 0.5$.

The identified proton and $p_{\text {fast }} y_{\text {lab }}$ distributions are combined in figure 7.5.3 for the two definitions of $\Delta \Phi$. Again there is remarkable agreement between $p_{\text {faot }}$ distributions comparing $\pi^{ \pm} \mathrm{Ne}$ to $\left(\bar{\nu}^{\prime} \mathrm{Ne}\right.$. After including identified protons, the low $y_{l a b}$ tail $\left(y_{l a b}<0\right)$ is higher for $\pi^{ \pm} \mathrm{Ne}$ than for $\left(\bar{\nu}^{\prime} \mathrm{Ne}\right.$, particularly in $\Delta \Phi(1)$ (figure 7.5.3(a)). There is a break in the $\Delta \Phi(1)$ distribution and none evident in the $\Delta \Phi(2)$ distribution. Furthermore the $\Delta \Phi(1)$ ylab distribution reaches a maximum of about $3.0-3.5$, whereas the $\Delta \Phi(2) y_{l a b}$ distribution only goes out to about $y_{l a b}=2$

The integrated contents of $\Delta \Phi(1)$ are significantly larged than the integrated contents of $\Delta \Phi(2)$ when evaluated in $\left({ }^{\nu}\right) \mathrm{Ne}$, but are equivalent within errors for $\pi^{ \pm} \mathrm{Ne}$. This difference is expected for $\left(\bar{\nu}^{\prime}\right.$ Ne because of the explicit flavor dependence of neutrino scattering.

We define $R_{\mathrm{p}}$ as the ratio of proton production given by $\Delta \Phi(1)$ to that given by $\Delta \Phi(2)$ :

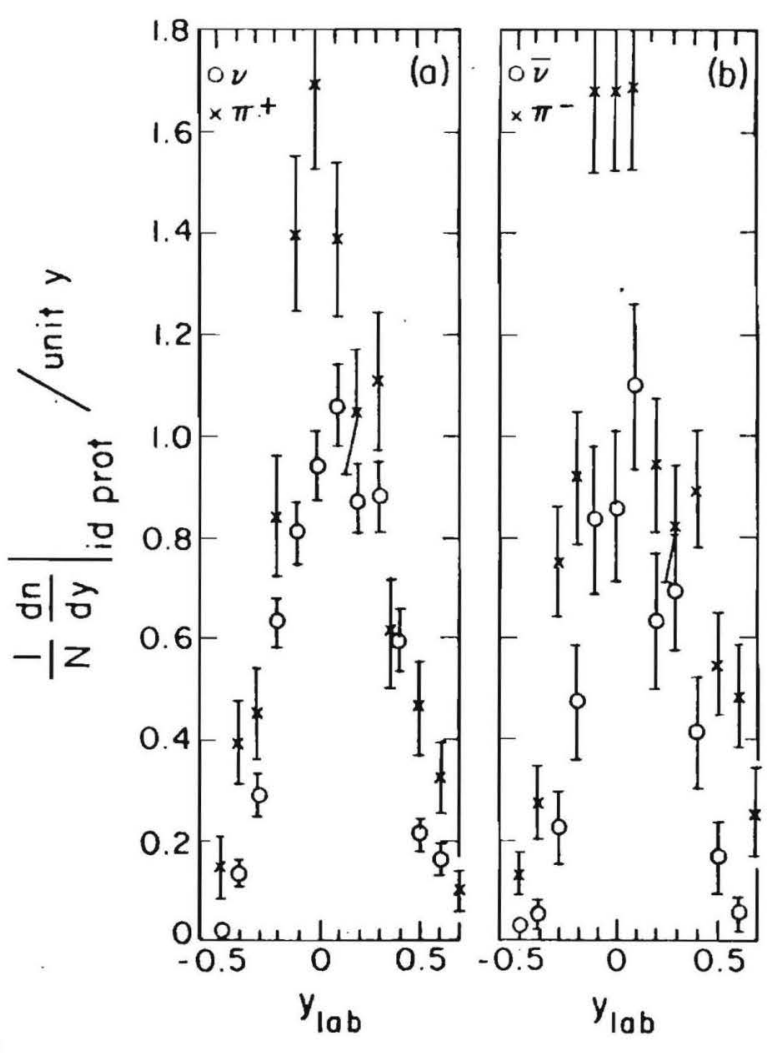

FIGURE 7.6.8.

The identifled proton viab distribution for $\pi^{ \pm} \mathrm{Ne}$ and $(\bar{\nu}) \mathrm{Ne}$ 


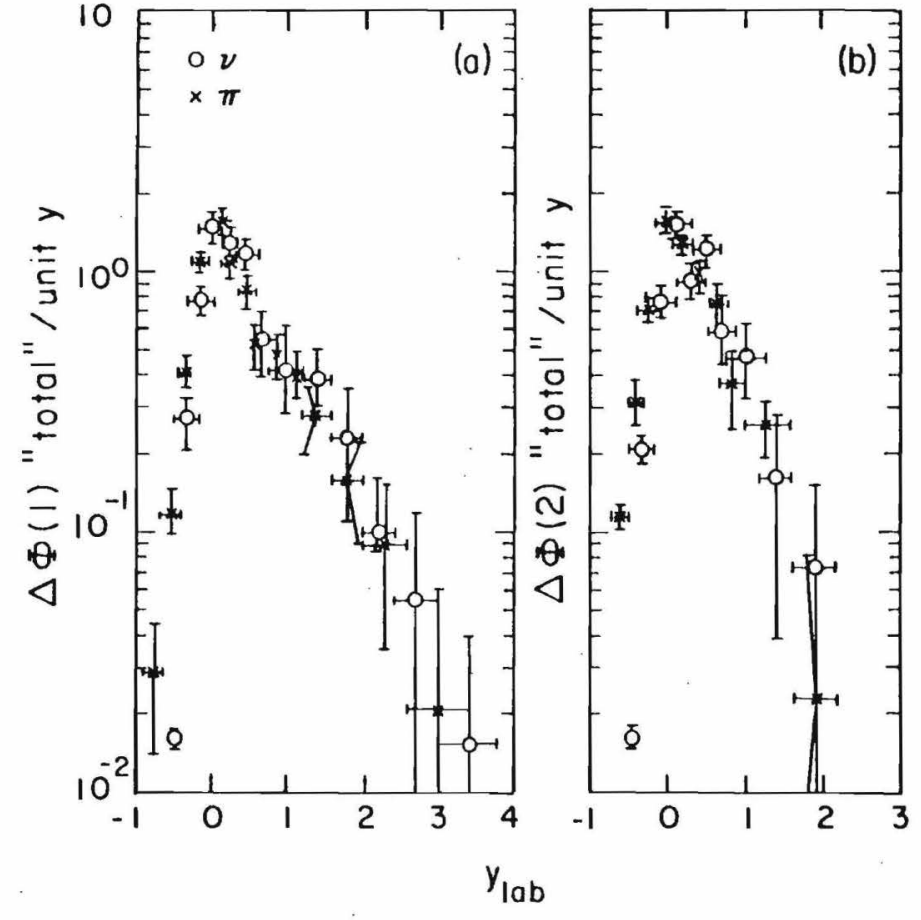

FIGURE 7.6.8.

The total proton yab distribution.

$$
R_{p} \equiv \int \Delta \Phi(1) d \theta / \int \Delta \Phi(2) d \theta
$$

Nuclear proton production from reinteraction effects cancels out of $\boldsymbol{R}_{\mathrm{p}}$ to first order since we expect equal amounts of nuclear production in $\Delta \Phi(1)$ and $\Delta \Phi(2)$. Furthermore, the isospin subtraction technique is sensitive only to valence quark scattering, because the $u \leftrightarrow d$ sy mmetry of the ocean implies that production from ocean scatters cancel in the subtraction.

$R_{p}$ provides, in some sense, a measure of diquark breakup in $(\bar{\nu}) \mathrm{Ne}$ For neutrino scattering, in the limit where diquark breakup is forbidden we expect $R_{p}=2: 1$, or an upper bound on $R_{p}$ of 2 . If we allow an arbitrarily large amount of diquark breakup then $R_{p}=1: 1$, or a lower bound on $R_{p}$ of 1 . All our data fall in this range. Our average value for $R_{p}$ for $W^{2}$ over the range $4 \leq W^{2} \leq 100$ is $R_{p}=1.44 \pm 0.14$.

Because by $u \leftrightarrow d$ symmetry $u d$ breakup does not enter into $\Delta \Phi$, only the $u$ and $d d$ diquark breakup probabilities need be known to compute $\boldsymbol{R}_{p}$. Assuming that these two probabilities are the same and iterating to two breakup levels, we can produce a crude estimate of the breakup probability $p_{u x}$ of uu diquark breakup by simply counting final states. This estimate gives $p_{u 4}=0.68 \pm 0.17$.

The data have been compared to the LUND model predictions. The LUND monte-carlo was run under conditions as closely approximating F546 as possible, including an equivalent neutrino energy spectrum, smearing tracks in momentum according to the measured E546 $\delta p$ distribution, with $\delta p$ taken as a function of $W^{2}$, generating track failures according to the measured E546 failure rates, and calling final state protons 
"identifled" only if they have $p<800 \mathrm{MeV} / \mathrm{c}$, with a linear extrapolation to $100 \%$ "nonidentifled" (ie treated as if they had a $\pi$ mass) at $p=1 \mathrm{GeV} / \mathrm{c}$. The resulting data were analyzed to extract $\Delta \Phi$ exactly as in the $\mathbf{E} 546$ case.

The LUND monte-carlo used as a target an isospin 0-weighted nucleon (ie $(p+n) / 2)$ and included no nuclear effects. The LUND prediction appears in figure 7.5.4, compared with the $(\bar{\nu}) \mathrm{Ne}$ data, for both $\Delta \Phi(1)$ and $\Delta \Phi(2)$. In $\Delta \Phi(1)$ LUND misses the tail for $y_{\text {leb }}<1$ but matches the high viab tail for viab $>2$ well. In $\Delta \Phi(2)$ LUND again matches the high viab tail for $y_{\text {lab }}>1.5$, but totally misses the $\nu_{\text {lab }}$ distribution for values below this. LUND predicts less than $5 \%$ Kaon contamination in the $\Delta \Phi$, and that the isospin subtracted distribution is essentially identical with the $p_{\text {faot }}$ distribution.

The LUND results evidently support the conclusion that $p_{\text {faot }}$ for $V_{i a b} \geq 1.5$ are mainly primary-vertex production products and not involved with nuclear effects (except possibly through energy degredation by rescattering) and that $p_{\text {faot }}$ for $y_{\text {lab }} \leqslant 1.5$ are mainly protons scattered out of the nucleus. The ratio of the two production mechanisms from figure 7.5.4 is roughly 1:1, consistent with the rough calculation of Section 8.3 .

Further evidence for this conclusion is presented in flgure 7.5.5, which compares the $\nu_{\text {lab }} p_{\text {feos }}$ distributions for those events classified as nonnuclear (NUCTST $=-2$ ) and those events classified as nuclear(NUCTST $=+2$ ) in $\Delta \Phi(1)$ (for a discussion of the NUCTST algorithm, see Chapter $5)$. There is a clear excess of $p_{\text {fast }}$ for nuclear events for $y_{l a b} \leq 1.5$, whereas the nonnuclear $p_{\text {faot }}$ are greater than or comparable to the nuclear $p_{\text {faot }}$ for $y_{l a b} \geq 1.5$.
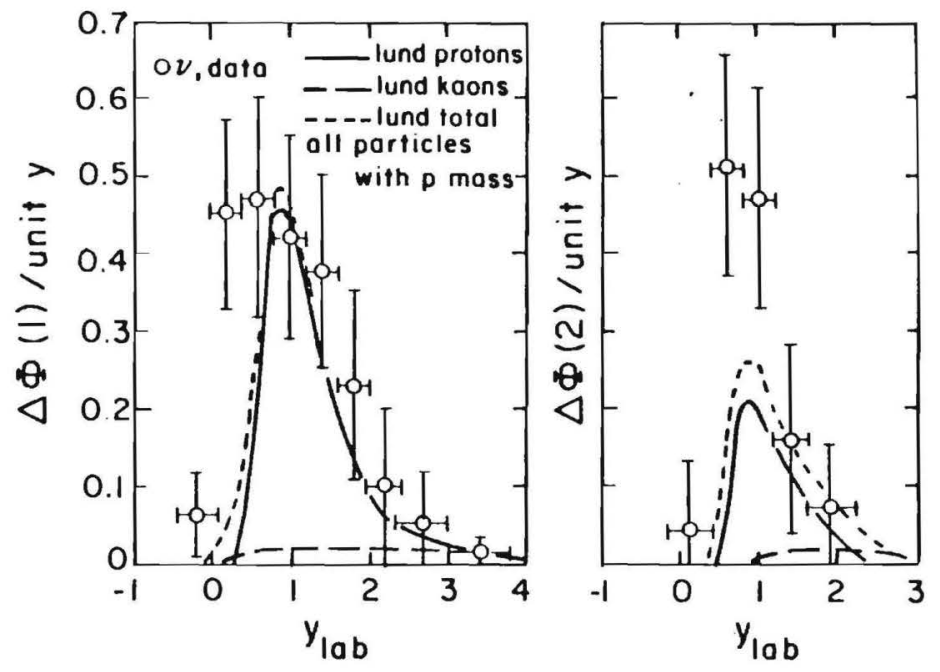

FIGURE 7.6.4.

The LUND model prediction compared with ${ }^{(} \bar{\nu}^{\prime} \mathrm{Ne}$. (s) $\Delta \Phi(1)$ and (b) $\Delta \Phi(2)$. (See text) 
219

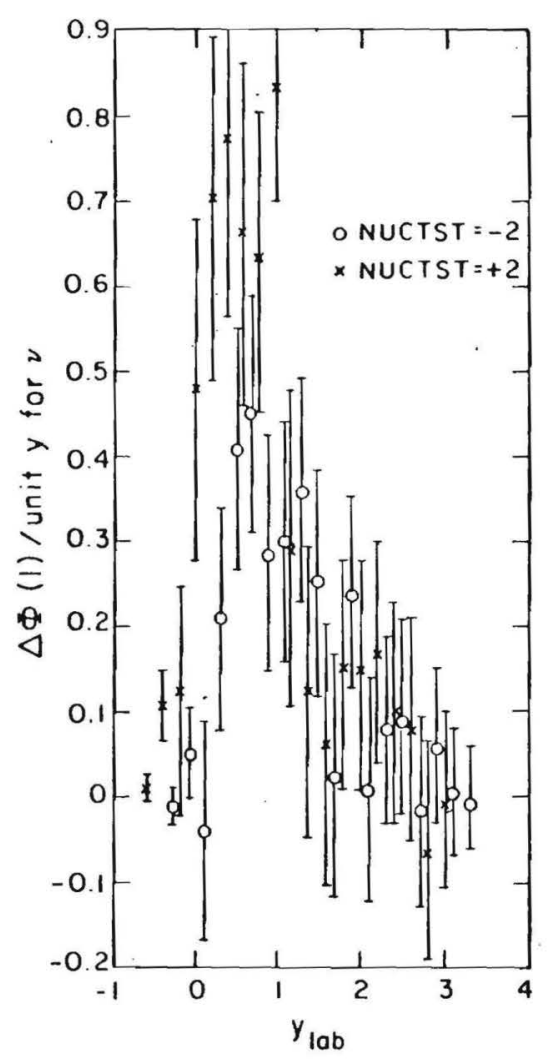

FIGURE 7.5.8.

Comparison of $p_{\text {faot }}$ Vies for nuclear (NUCTST $\left.=+2\right)$ and nonnuclear $(N U C T S T=-2)$ events for $\Delta \Phi(1)$.
220

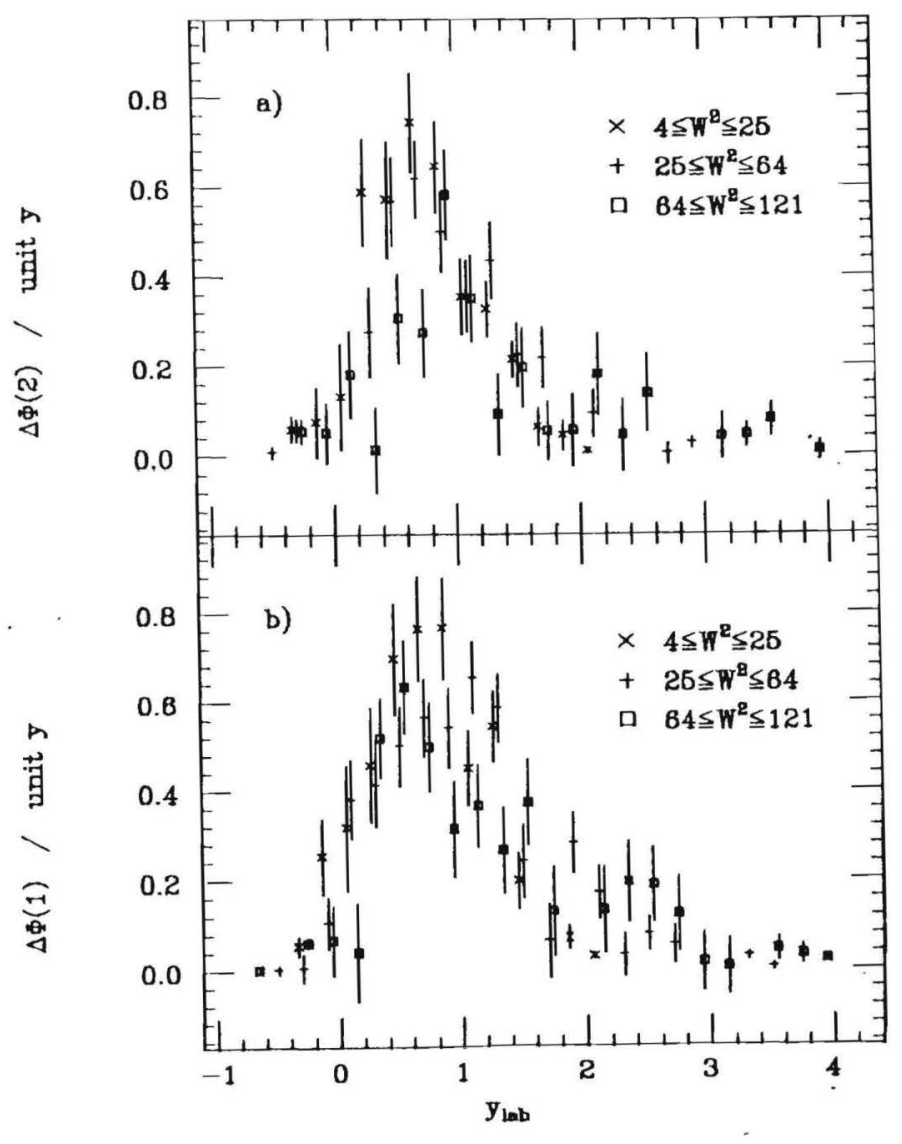

FIGURE 7.5.6.

The vlab $p_{\text {faot }}$ diatribution in $(\bar{\nu})$ Ne as a function of $W^{2} \quad$ (a) $\Delta \Phi(2)$ and (b) $\Delta \Phi(1)$. (See text) 
It is of interest to see how pfast production varies as a function of the event energy $W^{2}$. Figure 7.5.6 shows the extracted $y_{\text {lab }}$ distribution for $p_{\text {faet }}$ for three $W^{2}$ ranges: $4 \leq W^{2} \leq 25,25 \leq W^{2} \leq 64$, and $84 \leq W^{2} \leq 121$. Both $\Delta \Phi(1)$ and $\Delta \Phi(2)$ are shown.

The low $y_{\text {tab }}$ tail for $y_{\text {lab }} \leq 0.5$ stays essentially constant as $W^{2}$ increases, strong evidence that these $p_{\text {faet }}$ are nuclear effect protons. The total integrated number of $p_{\text {fast }}$ produced decreases very slowly as $W^{2}$ increases, decreasing only about $10-20 \%$ as $W^{2}$ increases by roughly a factor of 10 . Given that the number of $p_{\text {fast }}$ produced stays roughly the same as $W^{2}$ increases, the predominant effect is that the $y_{\text {tab }}$ peak is pushed down and flattened out and the maximum value of ylab reached grows by about 1 unit of rapidity. As $W^{2} \rightarrow 100 \mathrm{GeV}^{2} / \mathrm{c}^{4}$ the $y_{\text {lab }}$ distribution develops a long tail out to $y_{\text {lab }} \approx 3.0-3.5$.

This is probably mainly a phase space effect. As $W^{2}$ grows, the highest possible value of rapidity that can be reached $\left(\nu_{\max }\right)$ also grows. Figure 7.5.7 gives $y_{\max }$ as a function of $W^{2}$, where $y_{\text {maz }}$ was calculated as follows. The energy available in either hemisphere in the hadronic $\mathrm{CM}$ is $W / \mathbf{2}$, and $\boldsymbol{y}_{\max }$ occurs when $p_{T}=0$. Assume that the produced proton gets all the energy in the backwards hemisphere, then in the CM

$$
y_{\max }=\frac{1}{2} \ln \frac{W+\sqrt{W^{2}-4 m_{p}^{2}}}{W-\sqrt{W^{2}-4 m_{p}^{2}}} .
$$

But the transformation to the lab frame depends on $Q^{2}$, or alternatively $\nu$, which are not determined by fixing only $W^{2}$. However using the $\gamma_{c m}$ given in eqtn. 7.2.2 and below, and using the empirical relationship between $W^{2}$ and $\nu$ given in Section 7.2, the transformation can be calculated. The

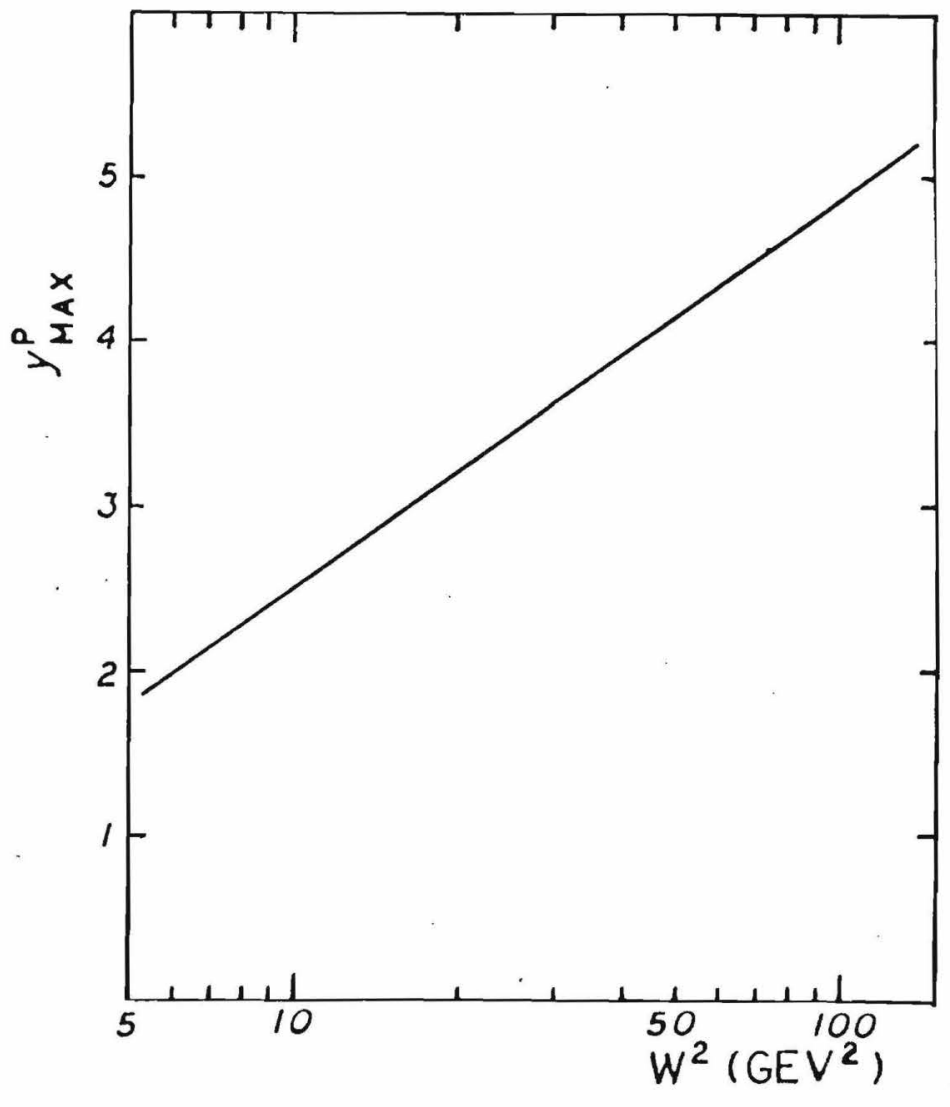

FIGURE 7.5.7.

The maximum proton lab rapidity for given $W^{2}$. 
result of the transformed $y_{\text {mas }}$ from CM to lab is the $y_{\max }$ plotted in figure 7.5.7.

The highest values of $y_{\text {lab }}$ reached for given $W^{2}$ in flgure 7.5.6 closely follow the behavior of $V_{\text {max }}$ given in figure 7.5.7, indicating that protons tend to be produced nearly up to the edge of the available phase space.

The integrated contents of each $W^{2}$-slice (ie the average number of $p_{\text {faot }}$ in $(\bar{\nu})$ Ne per event) are as follows:

$$
\begin{array}{cccc}
W^{2}: & 4-25 & 25-64 & 64-12 \\
\Delta \Phi(1): & 0.90 & 0.93 & 0.79 \\
\Delta \Phi(2): & 0.77 & 0.62 & 0.54
\end{array}
$$

\section{Notes for Chapter ?}

S.L. Adler, Phys. Rev. 135B, 963 (1964)

2. T. Sjöstrand, LU-TP-82-3 (1982)

3. D.R.O. Morrison, CERN/EP-83-159 (Contribution to the High Fnergy Physics Conference (Brighton, UK, 1983)), the WA21 and WA59 collaborations 


\section{ChAPTER 8}

\section{OTHER RESULTS}

\section{f 8.1. Chapter Overvlew.}

This chapter deals with other aspects of nuclear reinteraction effects in $\left(\bar{\nu}^{\prime} \mathrm{Ne}\right.$ and $\pi^{ \pm} \mathrm{Ne}$. Specifically, the topics dealt with are two-particle rapidity correlations, coherent scattering processes in $\left(\bar{\nu}^{\prime} \mathrm{Ne}\right.$, and a search for a $\Delta(1232)$ resonance signal expected to be present due to the presence of nonidentified protons, or $p_{\text {fast }}$ tracks.

Section 8.2 studies two-particle rapidity correlations in $\left(\bar{\nu}^{\prime} \mathrm{Ne}\right.$ and $\pi^{ \pm} \mathrm{Ne}$; that is, the tendency of produced particles to "bunch up" in certain regions of rapidity, or where the presence of one particle tends to exclude others from a region of rapidity. Clearly nuclear reinteractions will affect such correlations: Excess particles created in the target region of rapidity should create a strong correlation there, and in turn particles in the far forward or projectile region of rapidity will be depleted, leading us to expect a negative correlation in this ares. This effect is indeed observed and the full two-particle rapidity correlation matrix is compared between $\pi^{ \pm} \mathrm{Ne}$ and $\left(\bar{\nu}^{\prime} \mathrm{Ne}\right.$. In addition the rapidity gap distribution, where the rapidity gap is the $|\Delta y|$ between nearest neighbors in rapidity space, is presented for both systems. Unlike prior results for hadron nucleon ${ }^{[1]}$, these rapidity gap distributions can be fit well with a single exponential.

Section 8.3 presents results for coherent scattering in $\left(\bar{\nu}^{\prime}\right) \mathrm{Ne}$. In coherent scattering, the incident virtual $W^{ \pm}$interacts with the Ne nucleus as a whole ${ }^{[2]}$. A small signal is found in both $\nu \mathrm{Ne}$ and $\nu \mathrm{Ne}$ for 1-prong (with neutral tracks) events and 1-prong (without neutral tracks) events. The observed signal in $\mathrm{DNe}$ is comparable to the reported BEBC observation ${ }^{|3|}$ of coherent scattering, with corrections. Results for the E540 $\nu \mathrm{Ne}$ data, however, give a rate an order of magnitude smaller than either BEBC or E546 $D$ Ne. This difference is possibly due to the different $Q^{2}$ distributions of these samples, with the $\mathrm{E} 546 \nu \mathrm{Ne}$ data specifically chosen for high $Q^{2}$. Distributions in $|\boldsymbol{t}|,\left|t-t_{\min }\right|$, and invariant masses of the produced systems are presented.

Section 8.4 concerns a search for $\Delta^{++}$in the E546 $\nu$ Ne data. The proton for these $\Delta^{++}$decays would be a $p_{\text {faot }}$ track, and so not identifled as a proton. Such a signal is termed a $\Delta_{\text {faot }}^{++}$signal. Although calculations with the LUND monte-carlo ${ }^{[4]}$ indicate that such a signal should be visible after applying a series of specially-designed cuts to the data, the E546 $\nu$ Ne data does not have an observed signal, although a small "hint" of a signal is eventually found. The most likely reason for the non-observation of a $\Delta_{\text {fot }}^{++}$signal is that nuclear reinteractions are altering observed 
multiplicities, and $p_{\text {faot }}$ tracks due to nuclear reinteractions generate spurious $\Delta_{\text {fatt }}^{++}$combinations, masking the signal. (The LUND monte-carlo does not incorporate any mechanism to simulate nuclear reinteractions.)

\section{8.2. Two partiele rapidity correlations.}

This section studies two-particle rapidity correlations in $\pi^{ \pm} \mathrm{Ne}$ and $\left.{ }^{(} \bar{\nu}\right) \mathrm{Ne}$. Rapidity correlations are relevant to understanding "cluster" production and as such may be particularly relevant to understanding the dynamics of nuclear particle production. For example, the rapidity gap distribution (see below) has been found to depend on the target size ${ }^{[5]}$; that is, $A$. Also, to the best knowledge of the author the rapidity gap distribution has not been studied in neutrino-nucleus interactions before.

Analyzing the system in rapidity space breaks the reaction into three regimes: projectile, central, and target regions. The projectile region is at the most forward rapidities and presumably has properties associated with the beam; in like manner the most backwards rapidities define the target region, which has properties associated with the target. The central region is considered to be all rapidities in between and is also known as the "pionization" region - which indicates that it is an area where particles are produced by statistical equipartition of available energy, and therefore do not have much "memory" of the original properties of either the target or the beam. In light of this, we expect that nuclear effects will be strongly concentrated in the backwards hemisphere, and nearly absent from the far forward rapidity region.

A rapidity correlation exists if when we find a particle in some region of rapidity space, there is a greater chance of finding another particle in that same region of rapidity space than that we would expect from a uniform distribution of particles throughout the available phase space. Similarly anticorrelations exist when production of a particle in some volume tends to exclude other particles from that volume. Clearly in the finite energy world of experimentation, one often implies the other: If particles are forced out of some area of rapidity space, they clump up in some other area.

One can imagine several measures of rapidity correlations; the most commonly used one is the normalized two-particle correlation function

$$
R\left(y_{1}, v_{2}\right) \equiv \frac{N_{T} N_{2}\left(y_{1}, y_{2}\right)}{N_{1}\left(y_{1}\right) N_{1}\left(y_{2}\right)}-1,
$$

where $N_{T}$ is the total number of events in the snalysis, $N_{1}(y)$ is the number of particles with rapidity $y$, and $N_{2}\left(y_{1}, y_{2}\right)$ is the number of particle pairs with rapidities $y_{1}$ and $y_{2}$. If there are no particle pairs in some volume, then $R\left(y_{1}, y_{2}\right)=-1$. If all particles are distributed evenly in rapidity space, and we choose a rapidity interval size $\Delta_{y}$ from total rapidity volume $Y$, then of $n$ total particles per event there are $N_{T} \cdot n \delta y$ particles in $N_{1}\left(y_{1}\right)$ and in $N_{2}\left(y_{2}\right)$, where $\delta y=\Delta y / Y$.

Now two possibilities arise. Assume all particles we are studying are drawn from the same set; for example, all negative particles (in which case we call these particles "identical"). Then of $n$ particles per event, there are $2 \sum_{i-1}^{n-1} i=n(n-1)$ possible pairs per event, or $N_{T} n(n-1) \mathscr{V}^{2}$ pairs in interval $\Delta_{y}$ for the experiment. In this case the $N_{T}$ and $\delta y^{2}$ factors cancel, as they should, leaving $R\left(y_{1}, y_{2}\right)=n(n-1) / n^{2}-1=-1 / n$. This non-sero $R\left(y_{1}, y_{2}\right)$, even though we have excluded any correlation, 
is sometimes referred to as the Goldhaber identical particle effect. If we select a particular multiplicity chsnnel, such as all events with $n$ negative tracks, we can compensate for this effect by forming a new $R^{\prime}\left(y_{1}, y_{2}\right)=$ $R\left(y_{1}, y_{2}\right)+1 / n$.

Suppose alternatively the particles studied are drawn from two different, nonintersecting sets (such as positive tracks correlated with negative tracks). Then there are $n_{1} n_{2}$ pairs, given uniformly distributed particles, and $R\left(y_{1}, y_{2}\right)=0$ as we expect.

For the identical particle case with a constant number of particles $n$ produced per interaction, and given that all $n$ particles fall in the same $\Delta y$ interval, then all $n(n-1)$ pairs fall in this interval and

$$
R\left(v_{1}, v_{2}\right)=1-\frac{1}{n}
$$

We see that $R\left(y_{1}, y_{2}\right) \rightarrow 1$ as $n \rightarrow \infty$, ie $R\left(y_{1}, y_{2}\right)$ approaches 1 for perfect correlation and large multiplicity $\left(\boldsymbol{R}^{\prime}\left(\boldsymbol{v}_{1}, \boldsymbol{v}_{2}\right)=1\right.$ for perfect correlation in multiplicity channel $n$ in any case.)

Unusual events - events which are statistical fluctuations or which occur near the edge of rapidity phase space - may generate two-particle correlations greater than 1. Assume that there is only one event with a particle pair in some rapidity interval $\Delta y$, and assume that no other event has any particles in this $\Delta y$ interval. Then because the factor of $N_{T}$ in the numerator is not cancelled in the denominator, $R\left(y_{1}, y_{2}\right)$ diverges as $N_{T}$ for this interval.

The rapidity gap distribution also provides information on rapidity correlations. The rapidity gap $r$ is defined as the absolute value of the difference in rapidity between neighboring final-state particles, where the particles are ordered in rapidity. Clearly if all particles are distributed uniformly in rapidity then the rapidity gap diatribution is a delta-function; if short range correlations are more important, the rapidity gap distribution will peak for small $r$; long range correlations peak for large $r$. From the general principle of locality we expect that short range correlations will dominate.

Erwin et al. ${ }^{[0]}$ have studied $R^{--}\left(v_{1}, v_{2}\right)$ and $R^{+-}\left(v_{1}, v_{2}\right)$ for $\pi p$ and $p p$ at $100 \mathrm{GeV} / \mathrm{c}$, both for specific multiplicity channels with no multiplicity cut. They find that in all individual multiplicity channels all correlations with a central particle region particle are consistent with being zero, for both $R^{--}$and $R^{+-}$. We also find the central correlation in both $R^{--}$and $R^{+-}$in any given multiplicity channel is consistent with sero in both $\pi^{ \pm} \mathrm{Ne}$ and $(\bar{\nu}) \mathrm{Ne}$. There is some evidence of negative correlation between central-target and central-projectile regions in individual multiplicity channels, although statistics are marginal.

The following presentation of $R\left(v_{1}, y_{2}\right)$ is for charge-charge correlations $\left(\boldsymbol{R}^{c c}\right)$; that is, ignoring any distinction between identical and nonidentical particles. Equivalent analyses of our data for $R^{+-}$and $R^{--}$have been carried out, and both have a similar structure of the resultant correlation matrix as $R^{c c}$. Becsuse we are mainly interested in establishing trends within the correlation matrix, we use the large statistics $R^{e c}$.

Figures 8.2.1 and 8.2.2 present aspects of the rapidity correlation matrix $R\left(y_{1}, y_{2}\right)$. Clearly $R\left(y_{1}, y_{2}\right)$ is a function of the rapidity interval $\Delta y$ chosen. Too small a $\Delta \boldsymbol{V}$ and there will not be any correlations; too large a $\Delta y$ may not show correlations that exist on a smaller scale but are washed out when averaged over. This study uses a relatively tight correlation scale 


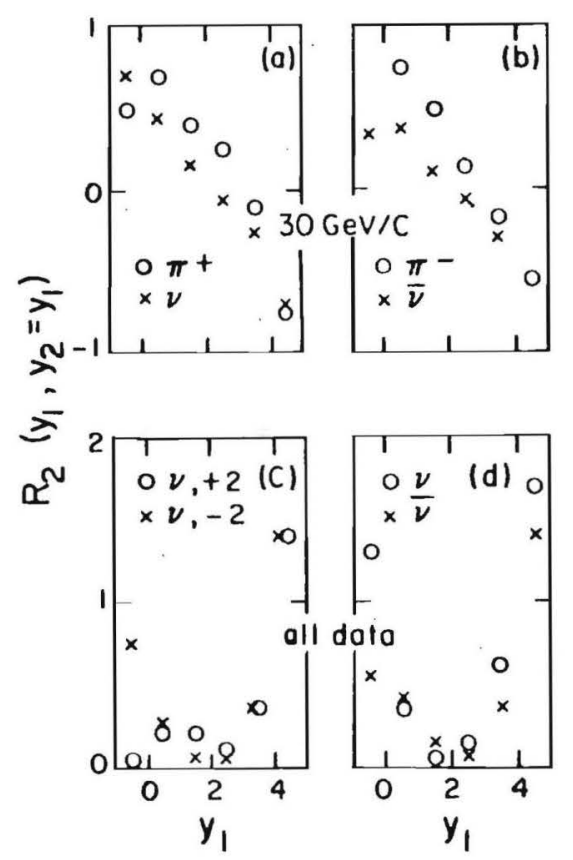

FIGURE 8.2.1.

Distributions for the diagonal of the two-particle correlation matrix,

$R\left(y_{1}, y_{2}=y_{1}\right)$. (a) $\pi^{+} \mathrm{Ne}$ at $30 \mathrm{GeV} / \mathrm{c}$ compared with $\nu \mathrm{Ne}$ at equiralent $W^{2}$. (b) $\pi-\mathrm{Ne}$ and $D \mathrm{Ne}$. (c) Nuclear compared with nonnuclear events in $\nu \mathrm{Ne}$. (d) $\nu \mathrm{Ne}$ compared with $\nu \mathrm{Ne}$, weighted to have equivalent $W^{2}$ distributions.

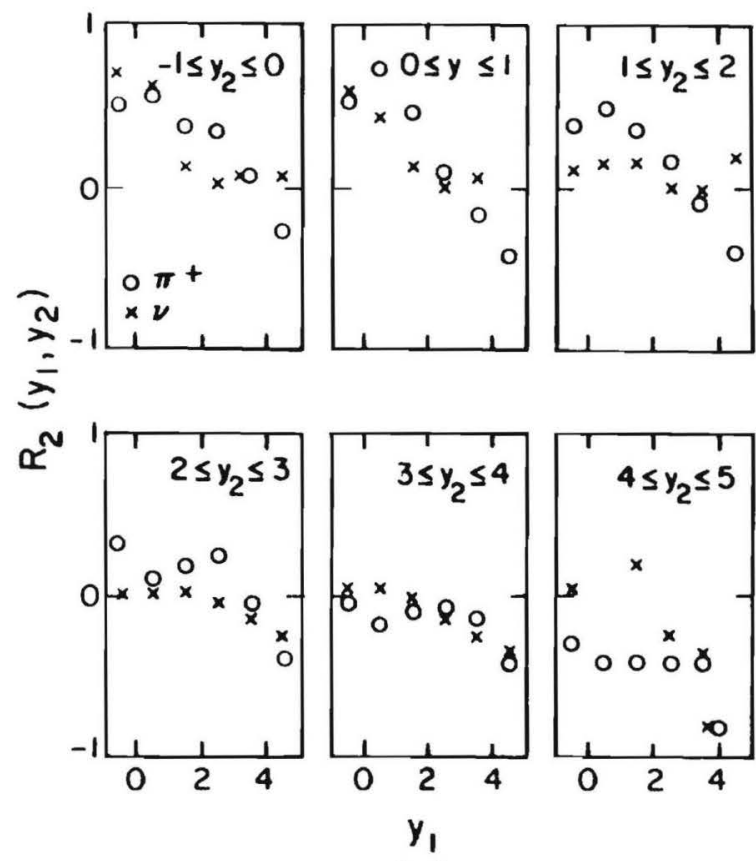

(o)
FIGURE 8.2.2.

Two particle correlations $R\left(y_{1}, v_{2}\right)$. (a) $\pi^{+} \mathrm{Ne} 30 \mathrm{GeV} / \mathrm{c}$ and equirslent $\nu \mathrm{Ne}$ 


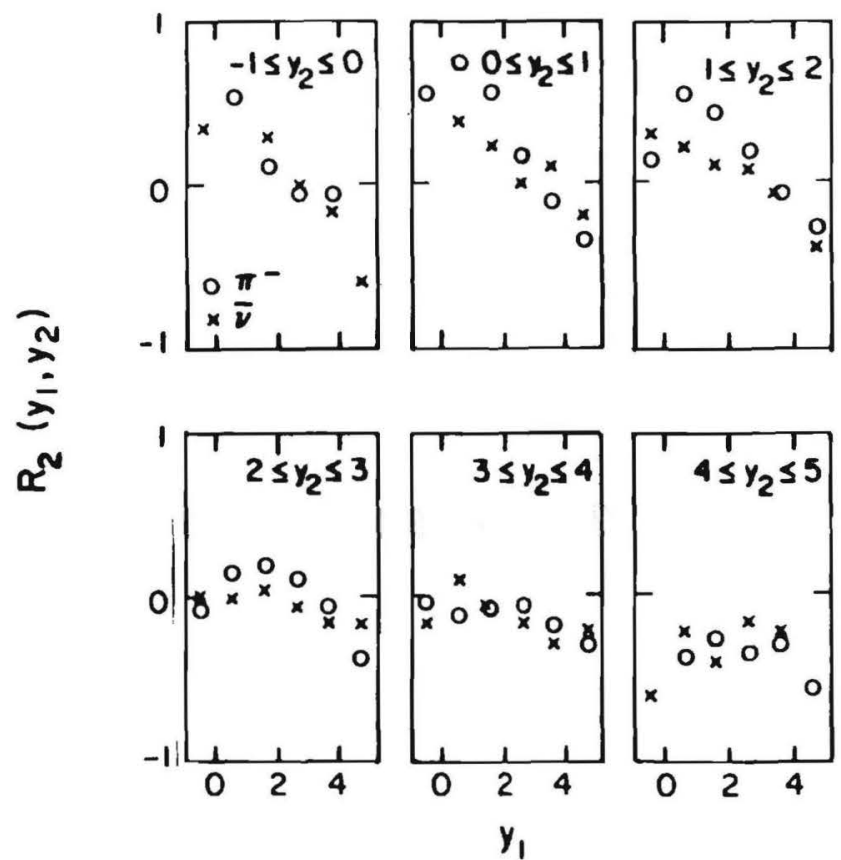

(b)

FIGURE 8.1.1 continued.

(b) $\pi^{-} \mathrm{Ne} 30 \mathrm{GeV} / \mathrm{c}$ and equiralent $\mathrm{DNe}$ of $\Delta y=0.25$, uses $y_{\text {lab }}$ as the rapidity variable to avoid any problems with $\mathrm{CM}$ transformations in neutrino interactions, and has the range $-1 \leq y_{\text {lab }} \leq 5$. To smooth out fluctuations for such a tight $\Delta y$, and to reduce the 576 correlation values resulting to a manageable number, the 16 values for each square unit of rapidity in $\left(y_{1}, y_{2}\right)$ space are averaged to a single value for that region.

Figure 8.2.1 gives several distributions in $R\left(y_{1}, y_{2}=y_{1}\right)$, ie the diagonal of the rapidity correlation matrix where the important short range correlations are manifested. Figure 8.2.1(a) compares $\pi^{+} \mathrm{Ne}$ at 30 $\mathrm{GeV} / \mathrm{c}$ and $\nu \mathrm{Ne}$ at equivalent $W^{2}$, and figure 8.2.1(b) compares the antiparticles. Both plots show a significant correlation in the backwards hemisphere where nuclear effects are expected, and drops to an anticorrelation in the most forward rapidities. $\pi^{ \pm}$Ne shows stronger short range correlations than $\left(\bar{\nu}^{\prime}\right) \mathrm{Ne}$; the trends and ralues in particle- and antiparticle-incident are essentially identical. The trend is clearly linear.

Such a result is not unexpected. Rescattering in the target nucleus should produce excess particles in the target region of rapidity, introducing a positive correlation. Particles far enough from the nucleus are $q \bar{q}$ pair production at essentially random $z$ and so we expect them to have $R\left(y_{1}, y_{2}=y_{1}\right) \approx 0$. The highest rapidities come either from the most forward quark or a "leading particlen quark. The most forward quark must be anticorrelated by energy conservation, and there can be only one leading particle quark contributed from the original system so it is by definition anticorrelated. The linear falloff might be construed as evidence for a string model with high tension at the nucleus end (a great deal of energy density) and low tension at the leading quark end. 
This linear falloff on the diagonal from target to projectile regions differs from the $\pi p$ and $p p R\left(y_{1}, y_{2}=y_{1}\right)$. The $\pi p$ and $p p$ diagonal is convex downward into both target and projectile regions, peaking in the central region ${ }^{[7]}$ (as expected from the symmetry). The backwards hemisphere correlation has been seen previously in p-Nucleus scattering in emulsion ${ }^{|5|}$. Our observation of this effect in the single-nucleus $\pi^{ \pm} \mathrm{Ne}$ and $\left(\bar{\nu}^{\prime}\right) \mathrm{Ne}$ systems, which are cleaner systems than sn emulsion mixture, confirm the backwards hemisphere correlation as a nuclear effect.

Figure 8.2.1(c) compares $R\left(y_{1}, y_{2}=y_{1}\right)$ for nuclear events (NUCTST $=+2)$ and nonnuclear events $($ NUCTST $=-2)$ in $\nu \mathrm{Ne}$, where statistics are strongest, for all $W^{2}$, (Such a plot for all $W^{2}$ should not be compared with other plots, since it represents an ensemble average over many different rapidity ranges, the net effect of which is unclear. However comparion with an event sample that has undergone the same ensemble average should be meaningful. This warning also applies to figure 8.2.1(d).) The figure shows that the correlation values are identical for the two samples in the forward region, where we expect the nucleus to have no effect, but differ in the most backwards region where the nucleus can play an important role.

Figure 8.2.1(d) compares $\nu \mathrm{Ne}$ and $\nu \mathrm{Ne}$ for all data. The distributions are similar with the $\nu \mathrm{Ne}$ parabola perhaps slightly broader than the $\nu \mathrm{Ne}$ parabola. In the figure, $\nu$ and $D$ have been weighted to have identical $\boldsymbol{W}^{2}$ distributions so that the same ensemble average will be performed for both systems (see chapter 5 for the weighting technique).

Figure 8.2.2 gives the full rapidity correlation matrix for $\pi^{ \pm} \mathrm{Ne}$ at $30 \mathrm{GeV} / \mathrm{c}$ and for $\left(\bar{\nu}^{\prime}\right) \mathrm{Ne}$ at equivalent $\boldsymbol{W}^{2}$. For low values of rapidity $\left(y_{l a b}<2\right)$ the peak of the correlation distribution tracks $y_{1}=y_{2}$ as expected from short range correlations, falling off away from the diagonal. As $y_{i a b}$ increases above $2, R\left(y_{1}, y_{2}\right)$ flattens out and indicates essentially no correlation. At the highest values there is some anticorrelation. For $\left(\bar{\nu}^{-} \mathrm{Ne}\right.$ $R\left(y_{1}, y_{2}\right)$ evidently flattens out sooner than for $\pi^{ \pm} \mathrm{Ne}$, and is generally lower than in $\pi^{ \pm} \mathrm{Ne}$. This behavior is consistent both with the increased "interaction length" expected in $\pi^{ \pm} \mathrm{Ne}$ compared to $\left(\bar{\nu}^{\prime}\right) \mathrm{Ne}$, and having an "extra" two quarks in $\pi^{ \pm} \mathrm{Ne}$ that can contribute to rapidity correlations.

The rapidity gap distribution is presented in figure 8.2 .3 for $\pi^{ \pm} \mathrm{Ne}$ and the corresponding $\left(\bar{\nu}^{)} \mathrm{Ne}\right.$ (in figure 8.2.3(a), the $\pi^{+} \mathrm{Ne} r$ distribution has been scaled by 2 in order to fit on the plot). The rapidity gap distribution peaks at small $r$ as expected for short range correlations and falls off exponentially. The slope for $\nu \mathrm{Ne}$ is identical to that for $\nu \mathrm{Ne}$, and the slope for $\pi^{+} \mathrm{Ne}$ is identical to that for $\pi^{-} \mathrm{Ne}$, but the slopes for $\left(\bar{\nu}^{-}\right) \mathrm{Ne}$ and $\pi^{ \pm} \mathrm{Ne}$ differ considersbly. Fits of $d n / d r$ to the form $n(r)=a e^{b r}$ are given in table 8.2.1.

As can be seen from the table, acceptable fits to the single exponential are obtained for all plots, and excellent fits for $\nu \mathrm{Ne}$ and $\pi^{-} \mathrm{Ne}$. This differs from previous results in hadron-nucleon ${ }^{[1]}$ which have required fits to a double expruential

$$
\frac{d n}{d r}=A e^{-B r}+C e^{-D r}
$$

because of a steeper peak at $r \leqslant 0.5$. Values of the slope for $p p$ scattering at $200 \mathrm{GeV} / \mathrm{c}$ are $\sim-3$ for the small-r peak and $\sim-1$ for the tail region, so the $\pi^{ \pm} \mathrm{Ne}$ values are consistent with this tail region in $p p$. The ${ }^{(} \bar{\nu}^{)} \mathrm{Ne}$ values are intermediate. 
237

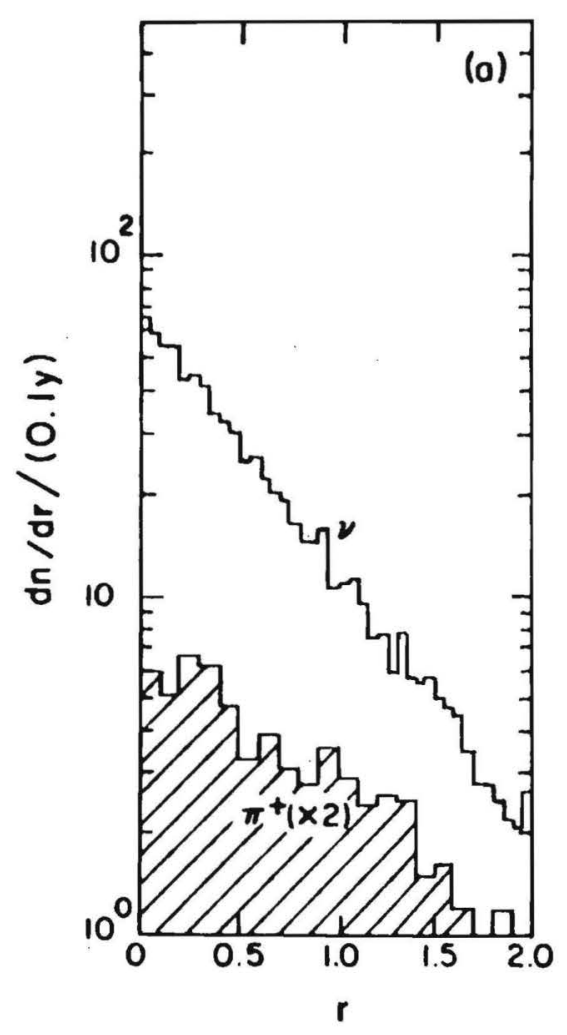

TIGURE 8.2.8.

The rapidity gap distribution. (a) $\pi^{+} \mathrm{Ne} 30 \mathrm{GeV} / \mathrm{c}$ and equiralent $\nu \mathrm{Ne}$ (the $\pi^{+}$distribution has been scaled by 2)
238

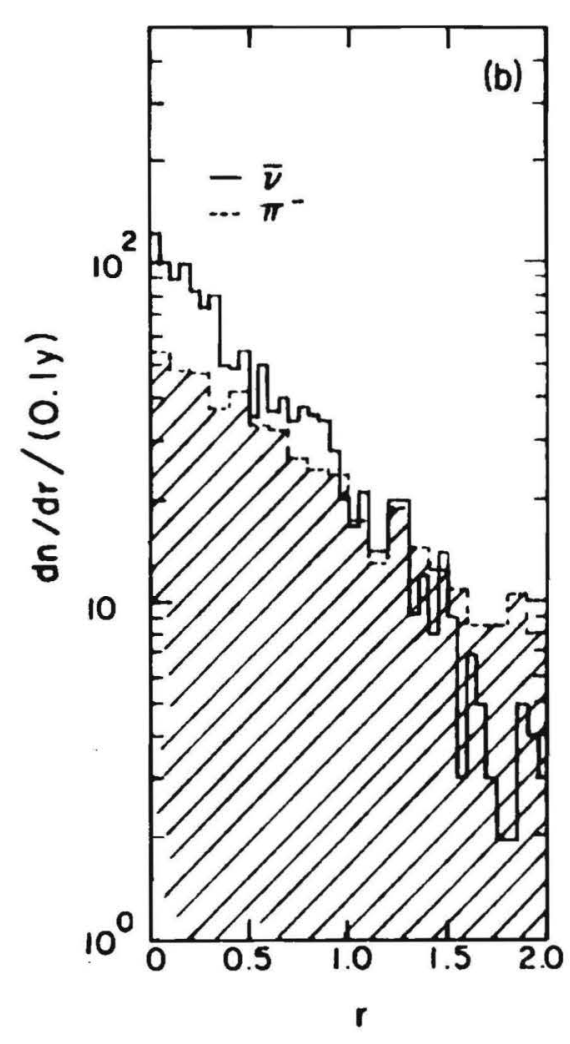

FIGURE 8.1.8 continued.

(b) $\pi^{-} \mathrm{Ne} 30 \mathrm{GeV} / \mathrm{c}$ and equiralent $D \mathrm{Ne}$ 


\section{TABLE 8.1.1.}

Fits of rapidity gap distributions to $n(r)=a e^{b r}$.

\begin{tabular}{|c|c|c|c|c|}
\hline \multicolumn{2}{|c|}{ reaction (E events) a } & 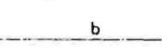 & $x^{2} /$ NDF & Prob \\
\hline$\checkmark N e(1879)$ & $695 \div 12$ & $-1.79 \pm 0.03$ & $31.2 / 38$ & 0.77 \\
\hline Ne $(362)$ & $120 \pm 5$ & $-1.77 \pm 0.07$ & $40.8 / 31$ & 0.11 \\
\hline${ }^{+}$Ne $(595)$ & $35 \pm 2$ & $-1.04 \pm 0.07$ & $49.0 / 39$ & 0.11 \\
\hline Ne $(583)$ & $59 \pm 3$ & $-1.08 \div 0.06$ & $38.3 / 38$ & $0.4 \mathrm{C} \quad \mathrm{y}$ \\
\hline
\end{tabular}

The absence of a small-r peak in these data is unexplained, although it should be noted that in experiments that use pseudorapidity $\eta$ as opposed to rapidity $y$, the pseudorapidity distribution will be pushed down and broadened with respect to the rapidity distribution, which could conceivably generate a tail of larger $r$ than would be seen in a rapidity distribution.

\section{j 8.3. Coherent Seattering.}

Coherent scattering is a process that has been extensively studied in hadronic interactions ${ }^{[8]}$, however it has only recently been observed in neutrino interactions ${ }^{[3]}$ (in the interaction $D \mathrm{Ne}$ ). This section analy zes the E546 neutrino data for evidence of coherent scattering (The WA51 $\pi^{ \pm} \mathrm{Ne}$ measurement data do not have adequate statistics to detect coherent scattering. Since coherent interactions typically account for a few percent of a given low multiplicity topological channel, and there are only $\sim 50$ events per incident pion sign per relevant topological channel for the $\pi^{ \pm} \mathrm{Ne}$ measurement sample, coherent processes are unobservable in the WA5I measurement sample.).

In coherent scattering the target remains in the same internal state both before and after the interaction. When the target is a nucleus, this means that the nucleus recoils as a whole and does not disentegrate or decay after recoil. Coherent scattering is closely related to elastic scattering; it is the same as the elastic process $k_{1}+p_{1} \rightarrow k_{2}+p_{2}$ except that the beam $k_{1}$ is allowed to "diffract" into a different particle or a multiparticle system. 


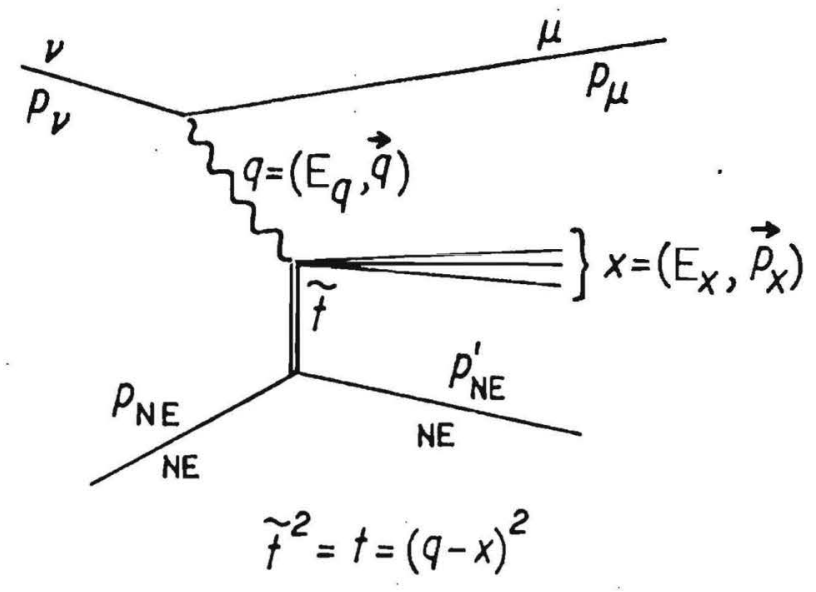

FIGURE 8.8.1.

Definition of quantities used in the coherent event analysis.
In neutrino coherent scattering the incident virtual $W^{ \pm}$with fourmomentum $q$ is treated as the beam and difracts to hadronic syatem $X$ by exchanging four-momentum $\tilde{t}$ with the Ne nucleus. Due to it's spinparity, the $W^{ \pm}$can couple to a vector meson $(p)$ or an axial-vector meson $\left(A_{1}\right)$, and via its divergence and PCAC to a pseudoscalar meson ( $\pi$ ). Since the interaction occurs as a whole on a large target, we expect coherent events to have low $Q^{2}$ and low $x_{B}$. The square of the four-momentum transfer from current to nucleus $(\tilde{t})$ is called $t$ and is the relevant kinematic invariant for the process. (For definition of the variables used in this section, see figure 8.3.1).

J. Lys ${ }^{(0)}$ has previously performed a search for coherent events in the E546 $\nu \mathrm{Ne}$ data. This analysis extends that search to $\nu \mathrm{Ne}$, compares our results to the recent $\mathrm{BEBC}$ coherent observation, and extends the analysis somewhat in form.

For the target to interact as a whole the relevant wavelength must be greater than the size of the target, ie $p \leq 1 / r_{N_{e}}$. Since $r_{N_{e}} \approx 3$ fermis, and taking a safety factor of three times this, this implies $p \leq$ $200 \mathrm{MeV} / \mathrm{c}$ for the Ne nucleus, or $t \leq 0.04 \mathrm{GeV}^{2} / \mathrm{c}^{4}$ for coherent events. Charge conservation requires the $X$ system to have the same charge as the incident $W^{ \pm}$, and since low momentum transfer implies low $X$ multiplicity, $X$ should have $N^{\text {prong }}=1,3$, or 5 . Events with an identified proton are excluded since Ne must remain intact, and events with an associated $\Lambda$ or $\Lambda$ are excluded because the heavy baryon must come from the nucleus.

To guarantee that the sign of the charge for charged tracks is correct, the cut $\delta p / p \leq 0.6$ is applied to all charge tracks (this corresponds 
to a $5 \%$ max probability of misassignment of the charge, see Chapter $5)$. Reconstructed neutral tracks must decay less than six lifetimes $\gamma \beta c r$ away from the vertex and be in an adequate constraint class. If an event contains a failing track for one of these ressons, the event is not used in the analysis. All tracks are assumed to be detected.

To compute $t$, note that for $p \leq 200 \mathrm{MeV} / \mathrm{c}$, the kinetic energy of the Ne nucleus $\left(T_{N_{e}}\right)$ is two orders of magnitude less than terms of order $p_{N e}$ and so can be safely ignored in comparison. Then

$$
\begin{aligned}
t & =\left(p_{N_{e}}^{\prime}-p_{N_{e}}\right)^{2}=\left(E_{N_{e}}-m_{N_{e}}\right)^{2}-\left|\overrightarrow{\mathrm{p}}_{N_{e}}\right|^{2} \\
& =T_{N_{e}}^{2}-p_{L N_{e}}^{2}-p_{T N_{e}}^{2} \\
& \approx-p_{L N_{e}}^{2}-p_{T N e}^{2},
\end{aligned}
$$

where $p_{N e}$ has been split into its longitudinal and perpendicular parts relative to the incident neutrino direction. Then

$$
\begin{aligned}
|t| & =p_{L N \mathrm{e}}^{2}+p_{T N e}^{2} \\
& =\left(E_{\nu}-p_{L}^{\mu X}\right)^{2}+\left(p_{T}^{\mu X}\right)^{2} \\
& =\left(E^{\mu X}-p_{L}^{\mu X}\right)^{2}+\left(p_{T}^{\mu X}\right)^{2}
\end{aligned}
$$

where momentum conservation $p_{L}^{\mu X}+p_{L N_{e}}^{\prime}=p_{L}^{\nu}=E^{\nu}$ has been used and again $T_{N e}$ has been ignored relative to the momenta involved. $\mu X$ refers to the $\mu+X$ system, ie all observed final state particles. The Ne nucleus is unobservable at these energies.

Also the quantity $t_{\min }=\min (|t|)$ is useful. $t_{\min }$ is the minimum value $|t|$ can assume for fixed current $q$, mass of the $X$ system $m_{X}$, and momentum of the $X$ system $\left|\overrightarrow{\mathbf{p}}_{X}\right|$ (in a given frame). We have

$$
-t=2\left(E_{q} E_{X}-|\mathbf{q}|\left|\boldsymbol{\beta}_{X}\right| \cos \theta\right)-m_{X}^{2}-m_{q}^{2},
$$

so $|t|$ has its minimum for $\theta=0$ for $-t(\theta=\pi$ for $t)$. Here an interesting difference occurs with a normal hardronic scatter; first, $-m_{q}^{2}$ is now a positive quantity, and second, there is no $-t_{\min }$ if we keep only the current and $m_{X}$ fixed, but let $\left|\overrightarrow{\mathbf{p}}_{X}\right|$ vary. Since the masses are fixed, only the term in parentheses of eqtn. 8.3.1 is minimized. If we define $E_{X}=k m_{X}$ with the constraint $k>1$, and also

$$
C=\frac{|\mathfrak{q}|}{E_{\mathrm{q}}}
$$

then this term is of the form

$$
2 E_{q} m_{X}\left(k-C \sqrt{k^{2}-1}\right)
$$

Now in normal hadronic physics have the constraint $C \leq 1$, in which case $k$ grows faster than $C \sqrt{k^{2}-1}$, and a term of the form 8.3.3 does have a minimum for varying $k$. But for the virtual current $W^{ \pm}, C>1$ and 8.3.3 has no minimum for varying $k$.

To compute $-t_{\min }$ for the case of fixed $\left|\overrightarrow{\mathbf{p}}_{X}\right|$, use the fact that $\theta=$ 0 for $-t_{\min }$. This implies if we compute $E_{N_{e}}$ in the hadronic CM and boost with a linear Lorentr boost to the lab frame (no rotations), then this gives $\left(E_{N e}\right)_{\min }$ in the lab frame, from which $-t_{\min }=\min \left(P_{N e}^{2}\right)$ is immediately calculated via $E=m+p^{2} / 2 m$.

$E_{N e}^{C M}$ can be written as a function of the particle masses and one energy (or equivalently $|p|$ ) variable alone because of the hadronic $C M$ constraint $\sum \overrightarrow{\mathbf{p}}=0$; the $|p|$ rariable is eliminated by rewriting it in combination with the particle masses to form a system mass. This gives

$$
E_{N \varepsilon}^{C M}=\frac{m_{N e X}^{2}+m_{N \varepsilon}^{2}-m_{X}^{2}}{2 m_{N e} X}
$$

where

$$
m_{N e X}^{2}=\left(E_{q}+m_{N_{e}}\right)^{2}-|\vec{a}|^{2}
$$


and where $E_{q}$ and $|\vec{q}|^{2}$ are obtained from $E_{q}=E_{\nu}-E_{\mu}$ and $|\vec{q}|^{2}=$ $\left|\overrightarrow{\mathbf{p}}_{\nu}-\overrightarrow{\mathbf{p}}_{\nu}\right|^{2}$.

The boost from the hadronic CM to the lab frame has

$$
\tau=\frac{E_{l a b}}{m_{c m}}=\frac{E_{q}+m_{N_{e}}}{m_{N_{e} X}},
$$

where $\mathrm{NeX}$ refers to the $\mathrm{Ne}+X$ system, and

$$
\left(E_{N e}^{l a b}\right)_{\min }=\gamma\left(E_{N e}^{C M}-\beta p_{N e}^{C M}\right) .
$$

The resulting $|t|$ and $\left|t-t_{\min }\right|$ distributions are shown in figure 8.3.2 for $\nu \mathrm{Ne}$ and in figure 8.3.3 for $\nu \mathrm{Ne}$. Also shown are mass plots for $\left|t-t_{\min }\right| \leq 0.04$ appropriate for $W^{ \pm} \rightarrow p^{ \pm}$in a coherent process.

Figure 8.3.2(a) shows the $|t|$ distribution for the number of charged hadronic tracks $=1,3$, and 5 and no neutral tracks in the event; figure 8.3.2(e) is the equivalent plot for the $\left|t-t_{\text {min }}\right|$ distribution. The 1-prong no neutral distribution is appropriate for $W$ coupling to $x$, the 3-prong no neutral distribution is appropriate for $W$ coupling to $A_{\mathbf{1}}$, say. The 1-prong no neutral distribution seems to show a peak at $|t| \leq 0.04$ of approximately 4 events, however this peak vanishes in the equivalent $\left|t-t_{\min }\right|$ distribution, so there is no strong evidence for it. Both the 3 and 5-prong distributions show dips at low $|t|$, so there is no evidence in this data of $W^{ \pm} \rightarrow A_{1}$. All the $\left|t-t_{\min }\right|$ distributions are compatible with exponential falloff, as they should be.

Figures 8.3.2(b) and 8.3.2(d) show the same distributions but for events with neutral tracks (ie $\gamma$ s) present. In particular, the 1-prong with neutrals distribution is appropriate for $W^{+} \rightarrow p^{+}$. This $\rho^{+}$channel shows peaks in both the $|t|$ and $\left|t-t_{\min }\right|$ distributions at $|t| \leq 0.04$. The 3 - and

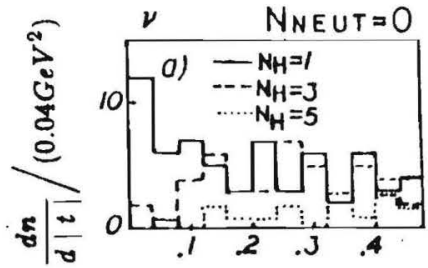

$|t|\left(G e V^{2}\right)$

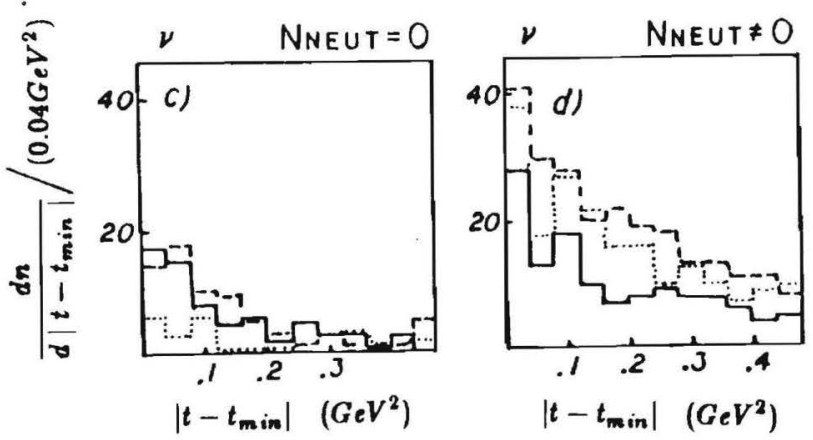

\section{FIGURE 8.2.2.}

Distributions in $|t|$ and $\left|t-t_{\min }\right|$ in $\nu \mathrm{Ne}$. (a) The $|t|$ distribution for 1-, 3 -, and 5 - prong channels, events with no neutrals. (b) The $|t|$ distribution for 1-, 3-, and 5- prong channels, events with neutrals. (c) The same as (a) for $\left|t-t_{\min }\right|$. (d) The same as (b) for $\left|t-t_{\min }\right|$. 


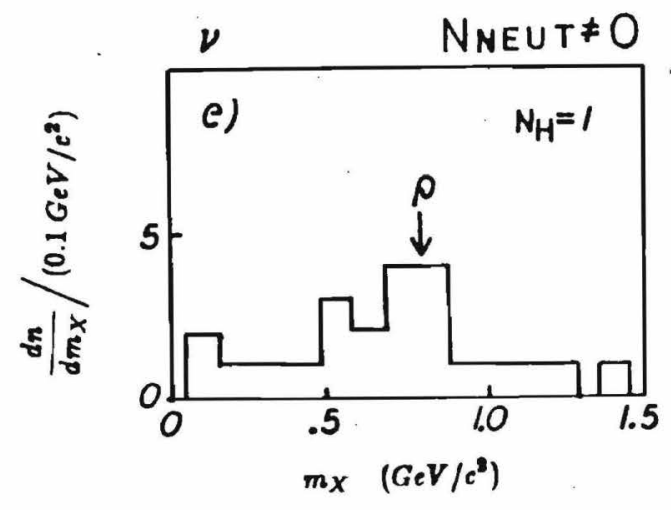

FIGURE 8.8.2 continued.

(•) The $m_{X}$ distribution for 1-prong events with neutrals and $\left|t-t_{\min }\right| \leq$ 0.04 .
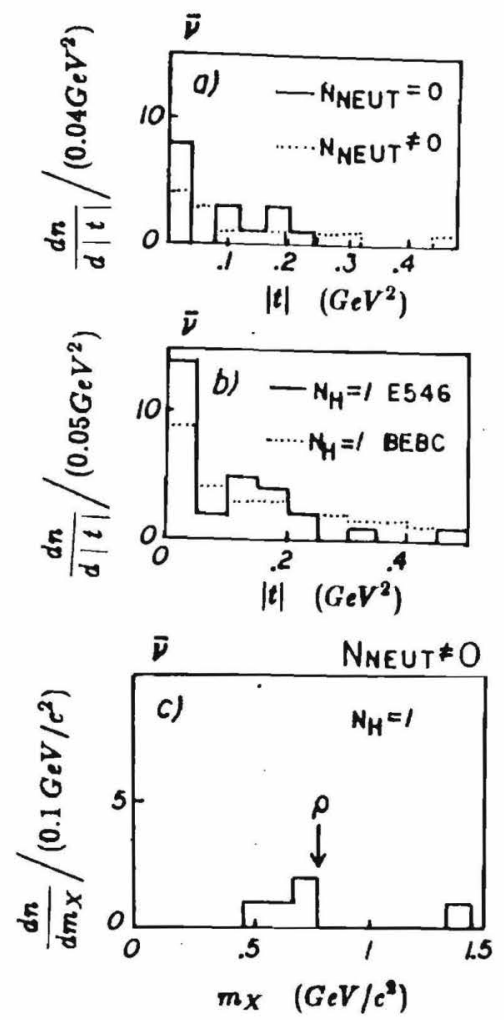

FIGURE 8.8.8.

Distributions in $|t|$ for $D \mathrm{Ne}$. (a) 1-prong events with and without neutrals.

(b) The combined 1-prong dist. compared with the equiralent BEBO dist.

(c) The $m_{X}$ distribution for 1 -prong events with neutrais and $\left|t-t_{\min }\right| \leq$ 0.04 . 
5 -prong channels with neutrals show dips at low $|t|$ and are compatible with exponential decline for $\left|t-t_{\min }\right|$, although there may be a slight excess in the three-prong with neutrals $\left|t-t_{\min }\right|$ channel. For the $\rho^{+}$ channel, the excess in both $|t|$ and $\left|t-t_{\min }\right|$ is $4 \pm 2$ events.

The mass of the $X$ system $\left(m_{X}\right)$ is plotted in figure 8.3.2(e) for the coherent $\left|t-t_{\min }\right|$ bin. There is indeed an excess of 4 events at the $\rho^{+}$mass. Since after momentum and other cuts there are 5578 events in the $\nu \mathrm{Ne}$ sample, this corresponds to a rate $W^{+} \rightarrow p^{+}$coherent in $\nu \mathrm{Ne}$ of $(0.07 \pm 0.035) \%$ uncorrected for scan/measure inefficiencies. The scan/measure corrected rate with a $\rho$ efflciency of 0.6 and a $\pi^{0}$ efficiency of 0.4 is $(0.3 \pm 0.2) \%$. If one takes the 4 "excess" events in the 1-prong no neutral channel as a coherent signal, then the $W^{+} \rightarrow \pi^{+}$coherent rate in $\nu \mathrm{Ne}$ is $(0.07 \pm 0.07) \%$.

Since there are only 981 events in the $\nu \mathrm{Ne}$ measurement sample after cuts, statistics are extremely limited. Therefore only the 1-prong with neutral and without neutral channels are plotted in figure 8.3.3 (the 3 and 5-prong channels in $|t|$ and $\left|t-t_{\min }\right|$ show no evidence of any coherent interactions, but this is statistics-limited, and so not a constraint on the $W^{-} \mathrm{Ne}$ system). The BEBC observation of coherent events was in the 1-prong $\overline{D N e}$ system (neutral and no neutral categories combined).

Figure 8.3.3(a) shows that there is indeed a large excess of events at $|t| \leq 0.05$ in the E546 $D \mathrm{Ne}$ data in the no neutral 1-prong category. There is no equivalent excess in the 1-prong with neutrals category. The combined (neutral + no neutral) 1-prong distribution is compared with the equivalent BEBC distribution in figure 8.3.3(b), where the BEBC distribution (with much greater statistics) has been normalized to have the same area as the E546 $\nu \mathrm{Ne}$ distribution. The excess of events in the coherent event bin for E546 $\nu \mathrm{Ne}$ is $8 \pm 3$ events. As shown in figure 8.3.3(e), there are $3 \pm 1 \rho^{-}$events in this bin, and correcting for scan/measure inefficiencies as above this corresponds to $12 \pm 7$ actual $\rho^{-}$events. This gives a $W^{-} \rightarrow\left(N^{\text {prong }}=1\right)$ coherent rate in $\nu \mathrm{Ne}$ of $(17 \pm 10) / 981=$ $(1.7 \pm 1.0) \%$ for the $\mathrm{E} 546$ data. The equivalent quoted BEBC rate is $(1.4 \pm 0.1) \%$.

Marage et al ${ }^{[3]}$ argue that there is little evidence of $W^{ \pm}$diffraction off a quasi-free nucleon in the Ne nucleus ${ }^{|10|}$. They note that in cases where more than $300 \mathrm{MeV} / \mathrm{c}$ nucleons are generated in the recoil, the $\mathrm{Ne}$ nucleus would break up, leading to a "coherent" signal in events with identified protons, where no such signal is observed (in either BEBC or E546 data). Since such events constitute such a small percentage of all events with identifled protons, this is not necessarily the case. They also note that the slope at low $|t|$ is about $30 / \mathrm{GeV}^{2}$, incompatible with the photoproduction value of about $\theta-8 / \mathrm{GeV}^{2}$ off nucleons. This is a more serious argument (the E546 data are not adequate to determine a slope).

An analysis equivalent to the above for $\left(\bar{\nu}^{\prime}\right) \mathrm{Ne}$ has been carried out assuming that the scatter occurs diffractively off a quasi-free nucleon in the Ne nucleus. In this case we expect that $|t| \leq 0.3$. As figures 8.3.4(a) and 8.3.4(b) show, there is a clear excess in the $\left|t-t_{\text {min }}\right|$ distribution for-1-prong, both with and without neutrals. The 3-prong distribution is compatible with exponential falloff. Furthermore, the $\left|\boldsymbol{t}-\boldsymbol{t}_{\min }\right| \leq \mathbf{0 . 3}$ 1-prong with neutrals distribution shows a clear $\rho$ peak for both $\nu \mathrm{Ne}$ and $\bar{\nu}$ Ne (figure 8.3.4(e)), although there is a background feature at $m_{X} \approx 0.4$ $\mathrm{GeV} / \mathrm{c}^{2}$. 


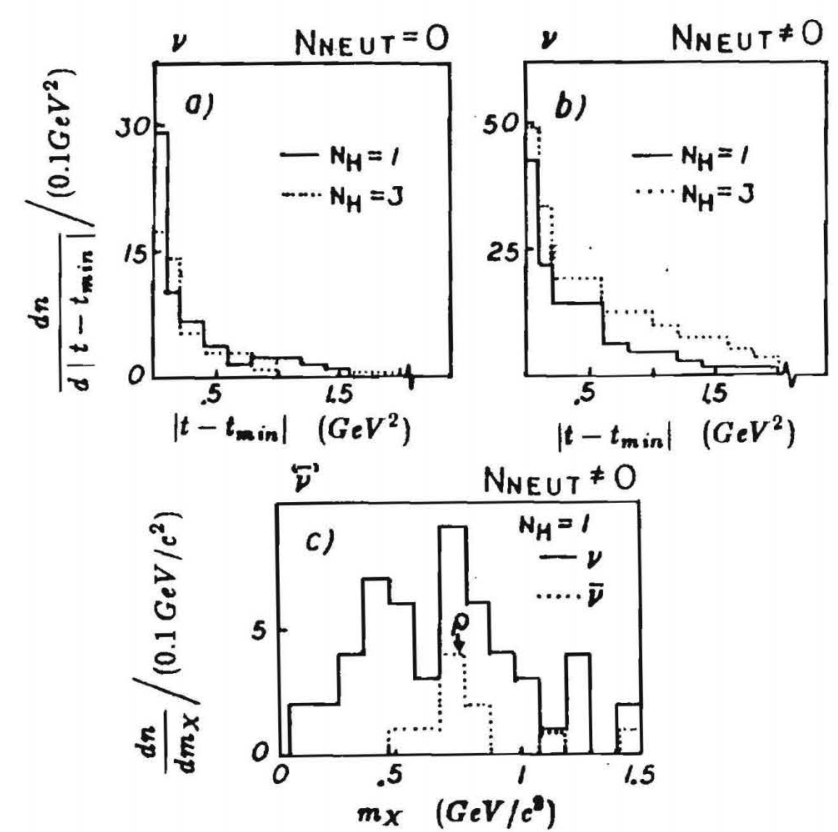

FIGURE 8.8.4.

Distributions in $\left|t-t_{\text {min }}\right|$ for single nucleon $W^{ \pm}$diffraction. (a) 1-prong and 8-prong distributions for events with no neutrals, $\nu \mathrm{Ne}$. (b) The same as (a) for events with neutrals. (c) The $m_{X}$ distribution for 1 -prong events with neutrals for $\left|t-t_{\min }\right|$ for $\left(\bar{\nu}^{\prime}\right) \mathrm{Ne}$.
In light of the extreme similarity of the two types of distributions, it would seem to be extremely difficult to disentangle coherent from diffractive-nucleon scattering in $\left(\bar{\nu}^{\prime}\right)$ Ne. I agree that the coherent interpretation is preferred, however.

The vast difference in $\sigma_{c o h}$ between the E54B $\nu \mathrm{Ne}$ and $\nu \mathrm{Ne}$ samples may be a $Q^{2}$ effect. The E546 $\nu$ Ne data are specifically selected for high $Q^{2}$, and $\left\langle Q^{2}\right\rangle$ in the $\nu$ Ne sample is significantly lower than in the $\nu \mathrm{Ne}$ sample. This higher $\left\langle Q^{2}\right\rangle$ may be suppressing coherent interactions in the $\nu$ Ne sample, since it is clearly necessary to have low $Q^{2}$ for coherent interactions. $\left\langle Q^{2}\right\rangle$ for E546 $\nu \mathrm{Ne}$ is 14.0 and only 9.1 for $\nu \mathrm{Ne}$. Furthermore, the skewness in $Q^{2}$ for $\nu \mathrm{Ne}$ is 1.01 compared to 1.86 for $\nu \mathrm{Ne}$, indicating that the $Q^{2}$ distribution is significantly more skewed toward lower $Q^{2}$ relative to its mean in $\nu \mathrm{Ne}$ than in $\nu \mathrm{Ne}$.

\section{8.4. Seareh for $\Delta_{\text {fait }}^{++}$in $\nu$ Ne.}

Conservation of baryon number dictates that st least one baryon will be present in the final state for deep inelastic neutrino scattering; other baryons may be produced in pairs via $6 \bar{b}$ production, but because lowestlying baryons have masses an order of magnitude larger than lowest-lying mesons, $b \bar{b}$ production is relatively rare at our experimental energies. If available energy is merely distributed according to the number of states available, then the phase space for meson production is at least two orders of magnitude greater than that for $b \bar{b}$ production. The upshot is that essentially all final-state baryons come directly from the initial-state baryon. 
Of course, in $\left(\bar{\nu}^{\prime}\right)$ Ne scattering, the nucleus provides a source of additional final-state bsryons vis secondary interactions, as discussed in prior sections. In the charge track distribution these $p_{\text {faot }}$ tracks will all be protons.

However flas state baryons from the initial interaction vertex can be any of the baryon states in figure 2.3.4. Ignoring charmed baryons as energetically forbidden at these experimental energies, there is still no a priori way to decide how these produced baryons will be distributed between the twenty-seven $q q q=10 \oplus 8 \oplus 8 \oplus 1$ possible flavor combinations. The simplest assumption is that produced baryons are distributed according to the number of isospin $\otimes$ spin states available (called $S U(\theta)$ weighting), but experimental results indicate this is far from the case and far fewer baryons wind up in the decuplet compared to the octet than the $S U(\theta)$ 2:1 prediction. Again this may be primarily an energy limitation, since decuplet baryons are more massive than octet baryons.

Since strange quark production is suppressed ${ }^{[11]}$ by the additional mass of the o relative to the $u$ and $d$, the most frequently produced baryons for the octet and decuplet will be the nucleon $N$ and the $\Delta(1232)$, respectively. If it is possible to identify the $\Delta(\mathbf{1 2 3 2})$ produced, then a mechanism exists for directly analyzing which $p_{\text {faot }}$ come from the initial interaction vertex and which $p_{\text {faot }}$ are nuclear rescatters, since the $\Delta \mathrm{s}$ come only from the initial vertex.

$\Delta(1232)$ production was studied using the LUND monte-carlo|4l, which has been customized to simulate the observed E546 momentum error and track failure rates as a function of $W^{2}$ and to simulate the incident $E_{\nu}$ spectrum. All tracks that don't have identifying signatures in the bubble chamber are assigned a pion mass; thus, protons with $p>1 \mathrm{GeV} / \mathrm{c}$ are treated as pions, protons with $p<800 \mathrm{MeV} / \mathrm{c}$ are treated as "identified" protons, and protons with intermediate momentum are linearly interpolated. All $K^{ \pm}$are treated as $\boldsymbol{x}^{ \pm}$, and resonances are decayed according to the appropriate Bright-Wigner distribution. The target for the monte-carlo was taken to be an isospin 0 nucleon, $(p+$ n)/2. Other than these E54B-specific modifications, the standard LUND algorithms are used to generate events, the key feature of which is an equiprobable distribution in $z_{C M}$ for creating $q \vec{q}$ pairs.

To determine the ratio of decuplet final-state baryons to octet finalstate baryons in E546 (which, as discussed above, will depend on the $W^{2}$ and $Q^{2}$ distributions of the experiment and so must be adjusted from experiment to experiment), the LUND monte-cario was tuned to reproduce relative rates of neutral strange particle production into octet and decuplet ${ }^{[12]}$. Neutral strange particles can be reliably identified in the bubble chamber and experimental rates can be reliably corrected to actual distributions, so they provide a good measure of the decuplet to octet ratio, $R_{D}$. In addition, such particles can only be produced at the initial vertex, so they measure $\left(R_{D}\right)_{\text {initial }}$, which is the quantity of interest here.

$R_{D}$ measured in this way is approximately $1 / 3$. Since the goal of this analysis is to attempt to identify $\Delta(1232)$ in the $\mathbf{E} 546 \nu \mathrm{Ne}$ data, in simulations for this analysis the LUND monte-carlo was run at the "conservative" $R_{D}$ of about half again this value, or about $1 / \mathbf{6}$.

The key features determined for $\Delta(1232)$ production in $\mathbf{E} 546 \nu \mathrm{Ne}$ from the LUND monte-carlo are as follows: (1) The four Delta charge 
states are produced in the ratio $\Delta^{++}: \Delta^{+}: \Delta^{0}: \Delta^{-}=8: 5: 3: 1$, (2) protons constitute about $15 \%$ of total stable final-state particles, (3) only about $10 \%$ of the final-state protons are "identified", the remainder having too high a momentum, and (4) about $1 / 5$ of all finsl-state protons are decay products of a $\Delta^{++}$.

Since $\Delta^{++}$is the most frequently produced $\Delta(1232)$, and since it decays solely to charged particles $\left(\pi^{+}+p\right)$, which are much more reliably detected than neutral particles in E546, $\Delta^{++}$was selected for the search. The difficulty in detecting $\Delta^{++}$is that it's mass occurs just above the threshold mass for $p \pi$, and so $\Delta^{++}$production just tends to push up the initial threshold peak without producing a noticeable bump on the background mass distribution, as it would on a smoother background.

Due to the large number of nuclear protons produced in E546 and the general poor efficiency for "identified" protons in E546, identified protons can not be used in this experiment to detect $\Delta^{++}$. Invariant mass distributions for $\mathrm{px}^{+}$in $\mathbf{E 5 4 6} \nu \mathrm{Ne}$ show no trace of any contribution from $\Delta^{++}$, and indeed tend to have a perfectly flat distribution after threshold, where one would expect a falling distribution if $\Delta$ production were a strong component in the plot. The flat background is an indication that any $\Delta^{++}$signal is buried beneath random contributions from nuclear protons $[13]$.

However only about $10 \%$ of the $\Delta^{++}$signal is accessible by using identified protons, and the vast bulk of the signal lies in minimum-ionizing " $\pi$ " assigned tracks. The presence of such a large signal raises the following possibility: If we form all possible unique invariant mass combinations of the " $\pi^{+n}$ assigned tracks taken two at a time, reassigning one of the
" $\pi^{+n}$ tracks as a proton, then in the $\Delta^{++}$region, the correctly assigned $\Delta^{++}$tracks will peak out on the background and form an identifiable bump. This assumes the background is well-controlled enough in this region for the bump to show.

$\Delta^{++}$identified by this technique are called $\Delta_{\text {faet, }}^{++}$"fast" because the proton from the $\Delta^{++}$is non-identified, or a $p_{\text {faet }}$ track.

Analyzing this system in the LUND monte-carlo indicates that the $\Delta_{\text {faot }}^{++}$signal is present, but still buried in the background; that is, there is no clean separation of the $\Delta_{\text {faot }}^{++}$signal from the background shape. Using the monte-carlo as a guide, a series of cuts were devised to enhance the $\Delta_{\text {faot }}^{++}$signal. If we define

$$
R_{\Delta}=\left(\frac{\text { Number of } \Delta^{++} \text {combinations }}{\text { All combinations }}\right)_{\text {after cuts }}
$$

then $R_{\Delta}$ provides a measure of the efficiency of the cuts.

The $R_{\Delta}$ for the uncut data in the LUND monte-carlo using the $\Delta_{\text {faat }}^{++}$ technique was 0.175 , evaluated over the mass interval $0.75 \mathrm{GeV} / \mathrm{c}^{2} \leq$ $m \leq 1.75 \mathrm{GeV} / \mathrm{c}^{2}$ (All subsequent analysis is performed in this interval). The three final cuts used were: (1) $N^{\text {ohower }} \in(4,6,8)$, with an $R_{\Delta}$ of 0.264 , (2) requiring that the momentum of the candidate reconstructed $\Delta_{\text {faot }}^{++}$satisfy $p<5.0 \mathrm{GeV} / \mathrm{c}$, with an $\boldsymbol{R}_{\Delta}$ of 0.272 , and (3) requiring that the momentum of both tracks used to reconstruct the $\Delta_{\text {faot }}^{++}$satisfy $p<$ $3.0 \mathrm{GeV} / \mathrm{c}$, with an $R_{\Delta}$ of 0.278 . The $R_{\Delta}$ of all three cuts in combination was 0.361 , or a total increase in signal to noise of about 2 for all cuts.

Using these cuts the LUND monte-carlo predicts a clearly visible bump in the $\Delta_{\text {faet }}^{++}$distribution, shown in figure 8.4.1. The figure also gives the background distribution, defined as those combinations not coming 


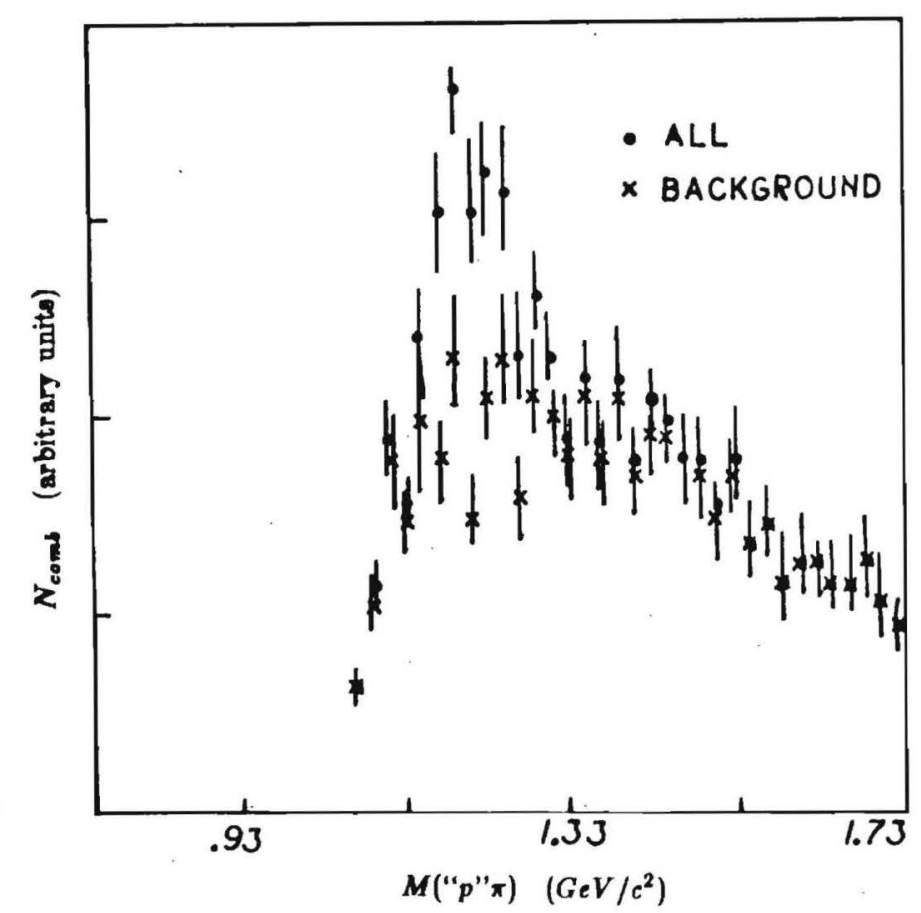

TIGURE 8.4.1.

The LUND prediction for the invariant mass distribution of $\Delta_{\text {feot }}^{++}$, with cuts (see text). The vertical scale is arbitrary. from an actual $\Delta_{\text {faet. }}^{++}$. An important feature to note is the linear tail in the mass region $1.3 \mathrm{GeV} / \mathrm{c}^{2} \leq m \leq 1.75 \mathrm{GeV} / \mathrm{c}^{2}$. Extrapolating this tail under the $\Delta_{\text {faot }}^{++}$signal is a reasonable estimate of the background for $\Delta_{\text {faot }}^{++}$i events above the extrapolation are taken as the $\Delta_{\text {faot }}^{++}$signal.

When the $\mathrm{E} 546 \nu \mathrm{Ne}$ data is analyzed with similar cuts, no deviation is seen from the linear background. A strong possible reason for this is that excess tracks generated by nuclear rescatters are sltering the event characteristics. Nuclear rescatters will contribute extra multiplicity, and $p_{\text {faet }}$ coming from nuclear rescatters will contribute spurious combinations tending to 8 mear out the $\Delta_{\text {faot }}^{++}$signal. To check this possibility the NUCTST algorithm was used to select out nonnuclear events from the total $\nu$ Ne sample, and these events were analyzed for $\Delta_{\text {faot }}^{++}$using all cuts.

The result is shown in figure 8.4.2. Two bins are now clearly above the linear tail extrapolation and provide at least a hint of a signal. However this "hint" certainly is not strong enough nor of the right width to term this an observation of $\Delta_{\text {faet }}^{++}$.

There is some evidence that there is some substance to the "hint": A sixth-order Legendre polynomial was used to fit the background distribution of the LUND prediction. The distribution in figure 8.4.2 was also fit by a sixth-order Legendre polynomial for two conditions: (a) excluding the two high bins, and (b) including the two high bins. For condition (a), the slope of the LUND background prediction and the 8.4.2 distribution agree exactly in the linear tail region; for condition (b), there is a significant mismatch of slopes.

If we use the condition (a) background, then the "hint" consists of 38.3 events above background. If we scale this by the LUND efficiency 


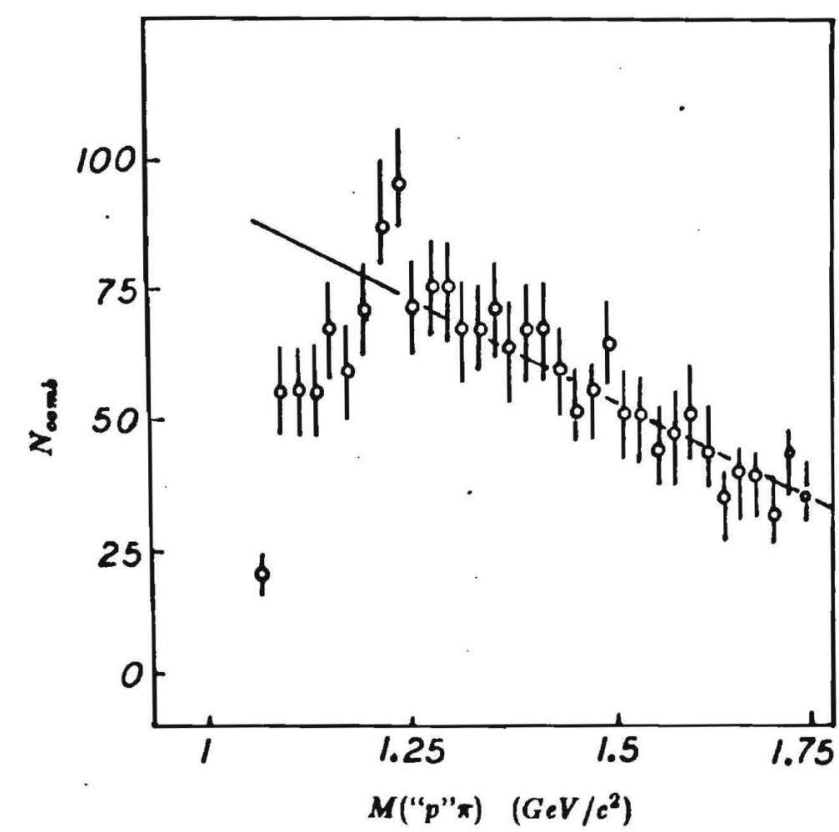

FIQURE 8.4.1. in these two bins, there are $83 \Delta_{\text {faot }}^{++}$in this interval. LUND predicts that the mass range of these two bins accounts for about $1 / 4$ of all $\Delta_{\text {faet }}^{++}$produced, and scaling again this corresponds to $328 \Delta_{\text {faot }}^{++}$. Finally, since LUND predicts that $\Delta^{++}$account for about $1 / 5$ of all primary vertex protons, scaling again yields a total of 1600 non-nuclear-generated protons. Since there are 4248 events in the event sample after using the NUCTST algorithm to select nonnuclear events, this corresponds to 0.38 primary protons per event. Using the isospin subtraction method on this same sample of events after weighting to insure that the $\nu \mathrm{Ne}$ and $\nu \mathrm{Ne}$ samples have equivalent $W^{2}$ distributions gives a value of 0.88 protons per event. These numbers are consistent with the earlier assertion that $p_{\text {faot }}$ are divided roughly 1:1 between primary-vertex $p_{\text {faot }}$ and nuclearrescatter $p_{\text {faot }}$.

Clearly the above calculation can not be taken very seriously in view of the questionable signal from which it started. In conclusion, no definite $\Delta_{\text {faot }}^{++}$signal is observed, although there is a small "hint" that a signal might be present. The probable reason for this is that nuclear effects mask the $\Delta_{\text {fa at }}^{++}$signal via multiplicity alteration and nuclear $p_{\text {faot }}$ generating spurious $\Delta^{++}$mass combinations. An alternative reason could be that $\Delta^{++}$production is far below the LUND prediction, but since this analysis was performed with a very conservative value of $R_{D}$, this seems unlikely. In addition, although the cut efficiency may not be as great as LUND predicts, the cuts used in the analysis are perfectly reasonable on general physical grounds, and so are expected to be effective in any circumstance. 
201

\section{Notes for Chapter 8}

1. D.R. Snider, Phys. Rev. D11, 140 (1975); G.F. Chew and A. Pignotti, Phys. Rev. 176, 2112 (1968); C. Quigg, P. Pirilä, and G.H. Thomas, Phys. Rev. Lett. 34, 290 (1975)

2. see, for example, K.S. Lackner, Nucl. Phys. B153, 528 (1979); D Rein and L.M. Sehgal, Nucl. Phys. B223, 29 (1983)

3 D. Marage et al "Observation of Coherent Diffractive Charged Current Interactions of Antineutrinos on Neon Nuclein, to be published.

4. T. Sjöstrand, LU-TP-83-3 (1982)

5. M.M. Aggarwal, 1.S. Mittra, and P.M. Sood, Phys. Rev. D29, 150 (1984)

6. J. Erwin et al., Phys. Rer. Lett. 33, 1443 (1974)

7. P.K. Sengupta et al., Phys. Rev. D20, 601, (1979)

8. V.G. Grishin, B.S. Yuldashev, and G. Jancso, Sov. J. Nucl. Phys. 14, 712 (1972); and references therein

9. J. Lys, "Search for Coherent Neon Events in E548", internal report of the E546 collaboration (1883)

10. for $\nu p$ diffractive production results, see J. Bell et al., Phys. Rev. Lett. 40, 1228 (1978); Phys. Rev. Lett. 41, 1008 (1978)

11. P.K. Malhotra and R. Orava, Z. Phys. C17, 85 (1983)

12. E.J. Wolin, "Neutral Strange Particle Production in High Energy Neutrino Interactions", PhD thesis, University of Washington,Seattle, USA (1984)
13. $\Delta^{++}$production has been observed in $\nu p$ interactions using identified protons. See J.P. Berge et al., Phys. Rev. D22, 1043 (1980) 


\section{BIBLIOGRAPHY}

\section{E.S. Abers and B.W. Lee, Phys. Rep. 9, no. 1, 1 (1973)}

H. Abramowics et al., Z. Phys. C-Particles and Fields Z, 199 (1981)

S.L. Adler, Phys. Rev. 135B, 963 (1964)

Y. Afek, G. Berlad, A. Dar, and G. Eilam, TECHNION-PH-76-87 (1976)

M.M. Aggarwal, I.S. Mittra, and P.M. Sood, Phys. Rev. D29, 150 (1984)

E. Albini et al., Nuov. Cim. 32A, 101 (1976)

J.C. Allaby et al., Sov. J. Nucl. Phys. 12, 295 (1971)

J. Ambjørn, P. Olesen, and C. Peterson, LU-TP-84-5 (March 1984)

C.D. Anderson and S. Neddermeyer, Phys. Rev. 23, 884 (1937)

\section{C.D. Anderson and S. Neddermeyer, Phys. Rev. 54, 88 (1938)}

B. Andersson et al., "A Monte-Carlo Simulation of Distributions of Recoiling Protons in High Energy Hadron-Nucleus Reactions", LU-TP-80-13 (1980)

B. Andersson, I. Otterlund, and E. Stenlund, Phys. Lett. 73B, 343 (1978)

B. Andersson, Nucl. Phys. B95, 237 (1975)

B. Andersson, Nuov. Cim. 38A, 329 (1977)

E.V. Anson et al., Phys. Lett. 31B, 241 (1970)

G. Arnison et al., Phys. Lett. 126B, 398 (1983)
R.G. Arnold et al., "Measurements of the A-dependence of Deep-Inelastic Electron Scattering From Nuclei", SLAC-PUB-3257 (1983)

J.J. Aubert et al., CERN-EP/83-14 (1983)

J.J. Aubert et al., Phys. Lett. 105B, 315, 322 (1981)

J.J. Aubert et al., Phys. Rev. Lett. 33, 1404 (1974)

J.E. Augustin et al., Phys. Rev. Lett. 33, 1406 (1974)

S.A. Azimov et al., Phys. Lett. 73B, 339 (1978)

S.A. Azimov et al., Phys. Rev. D23, 2512 (1981)

M: Banner et al., Phys. Lett. 122B, 476 (1983)

S. Barlag et al., Z. Phys. CW, 283 (1982)

R. Barloutad et al., Nucl. Phys. B176, 285 (1980)

S.Z. Belenkij and L.D. Landau, Suppl. Nuov. Cim. 3, 15 (1956)

J. Bell et al., Phys. Rev. D19, 1 (1979)

J. Bell et al., Phys. Rev. Lett. 40, 1226 (1978)

J. Bell et al., Phys. Rev. Lett. 41, 1008 (1978)

J.P. Berge et al., Phys. Rev. D22, 1043 (1980)

E.L. Berger, F. Coester, and R.B. Wiringa, Phys. Rev. D29, 398 (1984)

G. Berlad, A. Dar, and G. Eilam, Phys. Rev. D13, 181 (1976)

J.D. Bjorken and S.D. Drell, "Relativistic Quantum Mechanica" and "Relativistic Quantum Fielda", McGraw Hill (1964-1965)

A. Bodek and J.L. Ritchie, Phys. Rev. D23, 1070 (1981) 
A. Bodek and J.L. Ritchie, Phys. Rev. D24, 1400 (1981)

A. Bodek et al., "Electron Scattering from Nuclear Targets and Quark Distributions in Nuclei”, SLAC-PUB-3041 (1983))

A. Bodek et al., Phys. Rev. Lett. 30, 1087 (1973)

G. Bohm et al., "On the Correlations between Slow and Fast Protons in Iladron-Nucleus Interactions", contribution to the High Energy Physics Conference (Brighton, UK, 1983)

J.G. Bronson, "Proceedings 1981 International Symposium on Lepton and Photon Interactions at High Energies," edited by W. Pfeil, 279 (Bonn, 1981)

A. Buras et al., Phys. Lett. 17B, 251 (1973)

W. Buszs et al., Phys. Rev. Lett. 34, 830 (1975)

N. Cabibbo, Phys. Lett. 10, 513 (1983)

C.G. Callan and D.G. Gross, Phys. Rev. Lett. 22, 156 (1969)

A. Capella and J. Tran Thank Van, LP'TPE -80/7 (1980)

P. Carruthers and C.C. Shih, Phys. Lett. 127B, 242 (1983)

P. Carruthers, "Hadronic Multiplicity Distributions: Example of a Universal Stochastic Mechanism", talk given at Los Alamos Natl. Laboratory, Los Alamos, NM, USA

P. Carruthers, Phys. Lett. 114B, 169 (1982)

F. Cerulus, Nuov. Cim. 19, 528 (1961)

G.F. Chew and A. Pignotti, Phys. Rev. 176, 2112 (1968)

W. Czyz, "High Energy Particle Interactions with Nuclei”, Proceedings of the 1978 CERN School of Physics (Austerlitz-Zeist, Netherlands, 1978)
A. Dar and Fujio Takagi, Phys. Rev. Lett. 44, 788 (1980)

A. Dar and J. Vary, Phys. Rev. Dㅁ, 2412 (1972)

M. Derrick et al., Phys. Rev. D17, 1 (1978)

M. Derrick et al., Phys. Rev. D25, 624 (1982)

A. deShalit and H. Feshbach, "Theoretical Nuclear Physics", John Wiley and Sons (New York, 1974)

J.E. Elias et al., Phys. Rev. D22, 13 (1980)

J. Ellis et al., Nucl Phys. B131, 285 (1977)

M. Ericson and A.W. Thomas, Phys. Lett. 128B, 112 (1983)

J. Erwin et al., Phys. Rev. Lett. 33,1443 (1974)

E. Fermi, Z. für Physik 88, 161 (1934)

K. Fialkowski, "A User's Note on $e^{+} e^{-}$and $\nu p$ Average Multiplicities" (Feb. 1982)

P.M. Fishbane and J.S. Trefil, Phys. Lett 518 , 139 (1974)

P. Fritze et al., Phys. Lett. 96B, 427 (1980)

H. Geiger and E. Marsden, Phil. Mag. 25, 604 (1913)

H. Geiger and E. Marsden, Proc. Roy. Soc. A82, 495 (1909)

M. Gell-Mann, Phys. Lett. 8, 214 (1964)

S.L. Glashow, J. Iliopoulus, and L. Maiani, Phys. Rev. D2, 1258 (1970)

Yu. P. Gorin et al., Sov. J. Nucl. Phys. 18, 173 (1974) 
K. Gottfried, "High-Energy Physics and Nuclear Structure", edited by Gunnar Tibell (Uppsala, Sweden, 1973)

H. Grässler et al., Nucl. Phys. B223, 269 (1983)

V.G. Grishin, B.S. Yuldashev, and G. Jancso, Sov. J. Nucl. Phys. 14, 712 (1972); and references therein

D.J. Gross and F. Wilczek, Phys. Rev. Lett. 30, 1343 (1973)

H.B. Heilmann, Univ. of Bonn Internal Report WA21-INT-1 (1978)

S.W. Herb et al., Phys. Rev. Lett. 39, 252 (1977)

P.W. Higgs, Phys. Lett. 12, 132 (1964)

P.W. Higgs, Phys. Rev. Lett. 13, 508 (1964)

P.W. Higgs, Phys. Rev. 145, 1156 (1968)

"Proceedings of the 1879 International Symposium on Lepton and Photon Interactions at High Energies", edited by T.B.W. Kirk and H.D.I. Abarbanel, Fermilab, Ill.

Z. Koba, H.B. Nielsen, and P. Olesen, Nucl. Phys. B40, 317 (1972)

M. Kobayashi and K. Maskswa, Progr. Theor. Phys. 49, 652 (1973)

K.S. Lackner, Nucl. Phys. B153, 526 (1979)

L.D. Landau, "Collected Papers of L.D. Landau" (New York, New York 1968)

C.M.G. Lattes et al., Nature 159, 885 (1947)

Antoine-Laurent Lavosier, “Traité élémentaire de chemie” (1789)
T.D. Lee, "A Festschrift for Maurice Goldhaber”, edited by G. Feinberg, A.W. Sunyar, and J. Weneser (New York, New York Academy of Sciences, 1980)

T.D. Lee, "Particle Physics and Introduction to Field Theory", Harwood Academic Publishers (1981)

E.S. Lehman and G.A. Winbow, Phys. Rev. D10, 2962 (1974)

C.H. Llewellyn Smith, Phys. Lett. 128B, 107 (1983)

J. Lys, "Search for Coherent Neon Events in E546", internal report of the E546 collaboration (1983)

P.K. Malhotra and R. Oravs, Z. Phys. C17, 85 (1983)

D. Marage et al., "Observation of Coherent Diffractive Charged Current Interactions of Antineutrinos on Neon Nuclei”, to be published.

W. Marciano and H. Pagels, Phys. Rep. 36, 137 (1978)

A. B. Migdal, Rev. Mod. Phys. 50,107 (1978)

D.J. Miller and R. Nowak, Lett. Nuov. Cim. 13, 39 (1975)

G. Miller et al., Phys. Rev. D5, 528 (1972)

D.R.O. Morrison, CERN/EP-83-159 (Contribution to the High Energy Physics Conference (Brighton, UK, 1983)), the WA21 and WA59 collaborations

G. Myatt, CERN/EFCA/72-4 Vol. Il p. 117 (1972)

F. Niebergall, invited talk at Neutrino ' 82 Conference, Balatonfüred, Hungary (1982)

J. Orthel, PhD Thesis, University of California at Berkeley (1979)

M.L. Perl et al., Phys. Rev. Lett. 35,1489 (1975) 
M.L. Perl et al., Phys. Rev. Lett. 38, 117 (1976)

H.D. Politzer, Phys. Rev. Lett. 30, 1346 (1973)

C. Quigg, P. Pirilä, and G.H. Thomas, Phys. Rev. Lett. 34, 290 (1975)

D. Rein and L.M. Sehgal, Nucl. Phys. B223, 29 (1983)

F. Reines and C. Cowan, Phys. Rev. 113, 273 (1959)

R.M. Roberson et al., Phys. Rev. D21, 3064 (1980)

M. Roos et al., "Review of Particle Properties", LBL-100 Revised (UC34d)

E. Rutherford, Phil. Mag. 21, 689 (1911)

A. Salam and J.C. Ward, Nuo. Cim. 11, 588 (1959)

A. Salam and J.C. Ward, Phys. Lett. 13, 168 (1964)

D.N. Schramm, Physics Today 36, 27 (April 1983), and references therein

P.K. Sengupta et al., Phys. Rev. D20, 601, (1979)

T. Sjöstrand, LU-TP-83-3 (1982)

D.R. Snider, Phys. Rev. D11, 140 (1975)

E. Stenlund and I. Otterlund, LUIP 8108 (1981)

W.D. Walker, " $\pi$ Neon Collisions at High Energy, High Energy Physics 1980", XX Intl. Conf., Madison, Wis., USA, edited by L. Durand, I. Pendram, 77 (New York, Amer. Inst. Phys., 1981)

S. Weinberg, Phys. Rev. Lett. 19, 1264 (1967)
E.J. Wolin, "Neutral Strange Particle Production in High Energo Neutrino Interactions", PhD Thesis, University of Washington, Seattle, Washington, USA (1984)

A. Wroblewski, Acta Physica Polonica B4, 857 (1973)

A. Wroblewski, "Proceedings of the IIIrd Multiparticle Symposium", 140 (Zakapana 1972)

A.K. Wroblewski, "Topologic Cross Sections in $\pi p$ Interactions", Univ. of Washington, Visual Techniques Laboratory, Physics Note PHY-90 (1982)

W.M. Yeager et al., Phys. Rev. D18, 1294 (1977)

B.S. Yuldashev et al., Acta Phys. Pol. B9, 513 (1978)

B. Yuldashev, private communication

D. Zieminska et al., Phys. Rev. D27, 47 (1983) 
Eqtn. A.5 can be expanded by noting that given $p+q=1$, then by using the binomial expansion

$$
\begin{aligned}
1-(1-p)^{A} & =1-q^{A}=\sum_{\nu=0}^{A}\left(\begin{array}{l}
A \\
\nu
\end{array}\right) p^{\nu} q^{A-\nu}-q^{A} \\
& =\sum_{\nu=1}^{A}\left(\begin{array}{l}
A \\
\nu
\end{array}\right) p^{\nu} q^{A-\nu}+q^{A}-q^{A} \\
& =\sum_{\nu=1}^{A}\left(\begin{array}{l}
A \\
\nu
\end{array}\right) p^{\nu} q^{A-\nu}
\end{aligned}
$$

\section{Calculation of $\langle\nu\rangle$ in the impact parameter}

representation.

Using A.6 in A.5 gives

$$
\sigma^{A}(\overrightarrow{\mathbf{b}})=\sum_{\nu=1}^{A}\left(\begin{array}{l}
A \\
\nu
\end{array}\right)(\tilde{\sigma}(\overrightarrow{\mathbf{b}}))^{\nu}(1-\tilde{\sigma}(\overrightarrow{\mathbf{b}}))^{A-\nu}
$$
dependent superposition of nucleon wave functions, ie we take $|\boldsymbol{\Psi}|^{2}=$ $\prod_{i=1}^{\hat{A}} \rho\left(\mathbb{F}_{i}\right)$, where $\boldsymbol{\Psi}$ is the nuclear wave function and $\rho\left(\mathbb{F}_{i}\right)$ is the probability density for any given nucleon, normalized so that $\int \rho(\overrightarrow{\mathbf{f}}) d^{3} r=1$. Let $\sigma(\vec{b})$ be the nucleon cross section for a given reaction at impact parameter

$\vec{b}$. Then the corresponding nuclear cross section $\sigma^{A}(\vec{b})$ is $|1|$

$$
\begin{aligned}
& \sigma^{A}(\overrightarrow{\mathbf{b}})=\int d^{3} r_{1} \ldots d^{3} r_{A} \prod_{i=1}^{A} \rho\left(\overrightarrow{\mathbb{F}}_{i}\right)\left[1-\prod_{j=1}^{A}\left(1-\sigma\left(\overrightarrow{\mathbf{b}}-\mathbf{B}_{j}\right)\right)\right] \\
& =1-\int d^{3} r_{1} \ldots d^{3} r_{A} \prod_{i=1}^{A} \rho\left(\overrightarrow{\mathbb{f}}_{i}\right) \prod_{j=1}^{A}\left(1-\sigma\left(\overrightarrow{\mathbf{b}}-\overrightarrow{\mathbf{b}}_{j}\right)\right) \\
& =1-\int d^{3} r_{1} \ldots d^{3} r_{A} \prod_{i=1}^{A}\left[\rho\left(\overrightarrow{\mathbb{F}}_{i}\right)-\rho\left(\overrightarrow{\mathbb{F}}_{i}\right) \sigma\left(\overrightarrow{\mathrm{b}}-\overrightarrow{\mathbf{z}}_{i}\right)\right] \\
& =1-\prod_{i=1}^{A}\left[1-\int d^{3} r_{i} \rho\left(\mathbf{F}_{i}\right) \sigma\left(\overrightarrow{\mathbf{b}}-\overrightarrow{\mathbb{B}}_{i}\right)\right] \\
& =1-[1-\gamma(\mathbf{b})]^{\wedge}
\end{aligned}
$$

where we have defined $\tilde{\sigma}(\vec{b})=\int d^{8} r \rho(\overrightarrow{\mathbf{F}}) \sigma(\overrightarrow{\mathbf{b}}-\overrightarrow{\mathbf{b}})$, and $\vec{z}$ is the component of $\boldsymbol{P} \perp \hat{\boldsymbol{z}}$.

From eqtn. A.7 we see that $\tilde{\partial}(\overrightarrow{\mathrm{b}})^{\nu}$ is the probability of $\nu$ hits and $(1-\tilde{\sigma}(\vec{b}))^{A-\nu}$ is the probability of $A-\nu$ misses, so that in A.7 $\nu$ plays the role of the number of interactions in the nucleus. To compute $\langle\nu\rangle$, we take

$$
(\nu)=\frac{1}{\sigma^{A}} \int d^{2} b \sum_{\nu=1}^{A} \nu P(\nu, \vec{b})
$$

where

$$
P(\nu)=\left(\begin{array}{l}
A \\
\nu
\end{array}\right) \tilde{\sigma}(\overrightarrow{\mathrm{b}})^{\nu}(1-\tilde{\sigma}(\overrightarrow{\mathrm{b}}))^{A-\nu} .
$$

To evaluate A.8, note that defining

$$
\xi(x) \equiv \sum_{\nu=1}^{A}\left(\begin{array}{l}
A \\
\nu
\end{array}\right)(1-\tilde{\sigma})^{A-\nu z^{\nu} x^{\nu}}
$$

then

$$
\frac{d \xi}{d x}=\sum_{\nu=1}^{A}\left(\begin{array}{l}
A \\
\nu
\end{array}\right)(1-\tilde{\sigma})^{A-\nu} \tilde{\sigma}^{\nu} x^{\nu-1} \nu
$$


so that

$$
\begin{aligned}
\left.\frac{d \xi}{d x}\right|_{x=1} & =\sum_{\nu=1}^{\wedge} \nu P(\nu) \\
& =\left.\frac{d}{d x}[(1-\tilde{\sigma})+\tilde{\sigma} x]^{\wedge}\right|_{x=1} \\
& =A \tilde{\sigma}
\end{aligned}
$$

where the definition of the binomial expansion has again been used in A.12. Finally, substituting A.11-A.12 into A.8

$$
\langle\nu\rangle=\frac{1}{\sigma^{A}} \int d^{2} b A \partial(\vec{b}) ;
$$

yielding

$$
\langle\nu\rangle=\frac{A \sigma}{\sigma^{A}}
$$

where the normalisation of $\tilde{\sigma}(\overrightarrow{\mathrm{B}})$ must be such that $\int d^{2} b \tilde{\sigma}(\overrightarrow{\mathrm{B}})=\sigma$.

Eqtn. A.14 is the desired result.

\section{APPENDIX B.}

\section{Notation Conventions.}

This appendix describes the notation convention for four-rectors used in chapter 2.

Four-vector indices are represented by Greek letters (for example, $\boldsymbol{A}_{\mu}$ ). Vector or tensor indices of other dimensionality (in particular, three vectors) are represented by lower case Roman letters (for example, $p_{i}$ ). Four-vector indices take on values from 1 to 4 , where 1, 2, and 3 are the usual space components and 4 is the time component. The time component is always imaginary; ie $A_{\mathbf{4}}=i A_{0}$. In short, we choose a Euclidean metric with an imaginary 4 component to generate a Lorents norm.

Repeated indices are summed over. For example, $A_{\mu} A_{\mu}=A_{1} A_{1}+$ $A_{2} A_{2}+A_{3} A_{3}+(i)^{2} A_{1} A_{4}$.

The Pauli matrices are the 3 generators of $S U(2)$. They are 2-dimensional, traceless, orthogonal, Hermitian matrices. They may therefore be represented as a three-vector $\vec{f}=\left(\tau_{1}, \tau_{2}, \tau_{3}\right)=\left(r^{2}, r^{2}, \tau^{2}\right)$, where

$$
r_{1}=\left(\begin{array}{ll}
0 & 1 \\
1 & 0
\end{array}\right), \quad r_{2}=\left(\begin{array}{cc}
0 & i \\
-i & 0
\end{array}\right), \quad r_{3}=\left(\begin{array}{cc}
1 & 0 \\
0 & -1
\end{array}\right) \text {. }
$$


The Pauli matrices satisfy

$$
\begin{aligned}
\tau_{i} & =\tau_{i}^{\dagger} \\
{\left[r_{i}, \tau_{j}\right] } & =2 i \epsilon_{i j k} \eta_{k} \\
\left\{\tau_{i}, \tau_{j}\right\} & =2 \delta_{i j},
\end{aligned}
$$

where $[a, b]=a b-b a$ and $\{a, b\}=a b+b a$.

The $\gamma$ matrices are a higher-dimension representation formed from the Pauli matrices by taking products of matrices in the direct product space of the Pauli matrices and the two dimensional unit matrix, $I$. In the representation chosen here they are

$$
\begin{array}{ll}
\gamma_{1}=\left(\begin{array}{cc}
0 & -i r_{1} \\
i r_{1} & 0
\end{array}\right) & \gamma_{2}=\left(\begin{array}{cc}
0 & -i \gamma_{2} \\
i \tau_{2} & 0
\end{array}\right) \\
\gamma_{3}=\left(\begin{array}{cc}
0 & -i r_{3} \\
i \gamma_{3} & 0
\end{array}\right) & \gamma_{4}=\left(\begin{array}{cc}
I & 0 \\
0 & -I
\end{array}\right) .
\end{array}
$$

The $\boldsymbol{\gamma}$ matrices satisfy

$$
\begin{aligned}
\gamma_{\mu} & =\gamma_{\mu}^{\dagger} \\
{\left[\gamma_{i}, \gamma_{j}\right] } & =2 i \epsilon_{i j k} \sigma_{k} \\
\left\{\gamma_{\mu}, \gamma_{\nu}\right\} & =2 \delta_{\mu \nu},
\end{aligned}
$$

where the $\sigma_{i}$ are defined as the direct product $\sigma_{i}=r_{i} \otimes I$.

Both the Pauli and Gamma matrices satisfy $\left(r_{i}\right)^{2}=I$ and $\left(\gamma_{\mu}\right)^{2}=$ $I \otimes I$.

\section{VITA}

Claude David Rees

Bachelor of Arts, Colorado College, Colorado Springs, Colorado, 1970

Master of Science, University of Washington, 1978

Doctor of Philosophy, University of Washington, 1984 